\title{
MODELAGEM E SIMULAÇÃO DE HIDRÓGRAFAS
}

\author{
JOSÉ RAIMUNDO DE SOUZA PASSOS
}

Engenheiro Florestal

Orientador: Prof. Dr. WALTER DE PAULA LIMA

Dlecertepāo apresentede a Eecola superlor do Agrleultera "Lulz de Quolroz". de Unlvaraldado do sāo Paulo, para obtenpio do tltulo de Mestro drea do concontrapio: Cluncles Florestals

PIRACICABA

Estado de São Paulo - Brasil Janeiro-1993 
Ficha catalogretica preparada pela befáa de Livrcas da Divisalo de Biblioteca e Documentaćá - FCLQ/USF

\section{F289m}

Fassos, Jose fiaimundo de Souza 1993.

Modelagen e simulacao de hidrografas. Firacicaba. 1669.

Diss. (Mestre) - ESALQ

Eibliogratia

1. Bacia hidrográfica 2. Hidrografa - Modelagen 3. Hidrografa - Simulaça 4. Hidrologia florestal I. Escola Superior de Agricultura Luiz de Queiroz, Fi racicaba 


\section{MODELAGEM E SIMULAĈ̣̃o DE HIDRÓGRAFAS}

JOSE RATMUDD DE SOUZA PASSOS

Aprovada em: 19.03.1993

Comissão jul gadora:

Prof. Dr. Walter de Paula Lima ESALQNUSP

Prof. Dr. Hilton Thadeu Zarate do Couto ESALQNSP

Prof. Dr. Paulo Rodolfo Leopol do UNESP/BOTUCATU

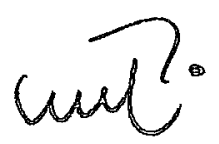

Prof. Dr. WALTER DE PAULA LIMA

Orientador 
A Gisela.

SURGE UMA NOVA VIDA 


\section{AGRADECI MENTOS}

- processo de elaboração de uma dissertação é único e gratificante. As etapas são multas, os detalhes inúmeros, mas a sensação 6 de criação.

No inicio uma ideia. O "objeto" ainda não tinha uma forma definida, mas certamente sua construção seria trabalhosa.

Ao longo deste processo há de se destacar a participação de algumas pessoas, cujas contribuiçőes foram importantes, compondo sem dúvida um "elenco" de primeira.

$$
\text { Já no periodo final da graduação na }
$$

Universidade Federal de Viçosa, recebi todo o apoio e estímulo para a construção deste "objeto", através do Prof. Paulo S'antana e Castro, incentivando o estudo de formas das curvas de vazão de microbacias.

Porem foi no Departamento de Ciencias Florestais ESALQUUSP, que a "construção" tomou forma. A 1 iberdade de trabalho, criação de conscléncia e confiança mútua, foram as marcas do estilo de orientação do Prof. Walter de Paula Lima, que juntamente com o incentivo, profissionalismo e determinação do prof. Hilton Thadeu Zarate do Couto na co-orientação estatistica, formaram um otimo elima de trabalho. Aos dois o meu muito obrigado! Após esta estrutura montada, faltava a materia prima para trabalhar. E nesta etapa que surge o Instituto Florestal de sao Paulo, que na pessoa do Eng. Florestal Haldir de Cicco, durante o perlodo do "incrivel" PIano Collor, demonstrou todo o interesse no projeto, contribuindo assim para a obtenção da "massa" de dados.

o trabalho foi iniciado e dois colaboradores merecem destaque no dia-a-dia de trabalho na ESALQ USP. O primeiro Prof. João Baptista, com objetividade e constante troca de ideias, muito contribuiu durante as etapas 
principais da construção. O segundo, o incansável Milton, que com seu conhecimento, paciencia e sotaque piracicabano, ofereceu grande ajuda na computação nos instantes de dificul dade.

Um outro destaque deste "elenco" , no que se refere a tabulação dos dados, revisão do texto a auxilio nos momentos dificeis, está na figura da minha esposa Liliane Vaz; a ela o muito obrigado.

Com o passar do tempo a situação ficou mais dificil. Estava trabal hando também em outra "construção", a Bahia Sul Celulose CBSCO, mas dentro da Companhia Suzano de Papel e Celulose CCSPCS. Neste instante, 0 apoio profissional e auxilio nos momentos mais importantes foi a marca dos Engos Florestais Luiz Cornachionni e Nelson Bastos.

Ja nesta etapa contava com o apoio de uma. equipe de SAS invejável da CSPC : Ewerton, Beth, Francisco e Maeda, obrigado pela ajuda!

Finalmente já na BSC em Teixeira de Freitas,foi questão de tempo para o termino. Agradeço á Bahia Sul Celulose, pelas oportunidades dadas para continuidade e conelusão deste trabalho. 


\section{SUMARIO}

Página

LISTA DE FIGURAS $\ldots \ldots \ldots \ldots \ldots \ldots \ldots \ldots \ldots \ldots \ldots \ldots$

LISTA DE TABELAS ....................

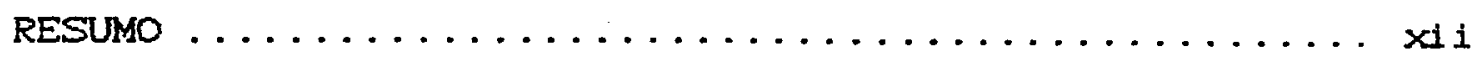

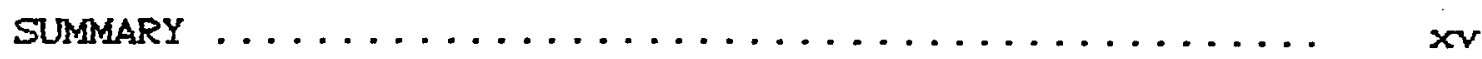

1. INTRODUÇAO .......................... 1

1.1. Caracterização do Problema .............. a

1.2. Objetivos....................... 3

2. REVISAO BIBLIOGRAFICA ................. 4

2.1. Ciencia e Hidrologia ................ 5

2. 2. Bacias Hidrográficas ................ 7

2.3. A hidrografa ..................... 12

2.4. A geração de deflúvio e seus mecanismos...... 16

2.5. Modelos hidrol bgicos - Classificação escol ha ........................ 20

3. O SISTEMA JOHNSON DE DISTRI BUI GXXO ............ 28

3.1. Fundamentos do Sistema Johnson

de Distribuiçåo ................... 29

3.2. Os sistemas ...................... 30

3.2.1. Sisteme $S L$................ 38

3. 2. 2. Sistema SB ..................40

3. 3. 3. Sistema su $\ldots \ldots \ldots \ldots \ldots \ldots \ldots . \ldots \ldots$

3. 4. Aplicaçes ... . . . . . . . . . . . . . . 44

4. METODOLOGIA ....................... A5

4.1. Área experimental - O sistema ...........46

4.1.1. Localização .................... 46

4.1.2. Relevo e caracteristicas físicas ..... 46

4.1.3. Solo ..................... 50

4.1 .4 . vegetaçå ................... 50

4.1.5. Clima ......................50

4. 2. Fundamentos do método utilizado ..........53 
4.2.1. Hipótese básica ............. 53

4. 2.2. Identificação e caracterizaçăo das chuvas - A entrada ......... 53

4.2.3. Identificação e caracterização das hidrografas - A salda ........ 57

4. 2. 4. Modelagem da hidrografa ...........58

5. RESULTADOS E DISCUSSAO ................... 63

5.1. Os dados de precipitação e vazão .......... 64

5.1.1. As chuvas ................. 64

5.1.2. As hidrógrafas .............. 71

5.2. PASH - Programa de Ajuste e Simulaçăo de Hidrografas .............. 77

5.2.1. Módulo "J" .................77

5. 2. 2. Módulo "A" ................. 78

5.3. A Modelagem ..................... 84

5.3.1. MSHJ - Modelos de Simulação de Hidrografas com base no Sistema Johnson de Distribuição .......... 84

5.3.2. MSHA - Modelos de Simulação de Hidrógrafas de Altura de 1 ámina d'água .... 86

5. 4. A simulação - Vali dação dos modelos ........ 91

6. CONCLUSOES . . . . . . . . . . . . . . . . . 117

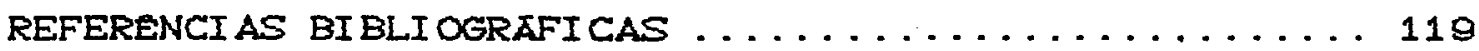

APENDICE 1 - As chuvas ...................... 131

APENDTCE $z$ - As hidrografas ............... 139

APENDICE 3 - Valores de "Ra" ................ 148.

APENDICE 4 - Rotinas do PASH CIinguagem SAS ....... 153 
Figura 1. Entendimento, prediça e controle ....... 6

Figura 2. Bacia hidrografica e seus componentes ..... 9

Figura 3. Representação de uma bacia hidrografica como um sistema ...................... 10

Figura 4. Componentes da hidrografa ............. 13

Figura 5. Mecanismos de geração do runoff ......... 21

Figura' 6. Classificação dos processos hidrologicos proposto por cHow .................. 22

Figura 7. Esquema de classificação de modelos hidrol bgicos proposto por CLARK ......... 24

Figura 8 . Sistema de translação do Sistema Johnson de Distribuiça ................... 31

Figura 9 . Representação de algumas distribuiçós no plano formado pelos coeficientes de assimetria e curtose ............... 35

Figura 10. Algumas possiveis distribuiç̧es apresentadas pelo Sistema Johnson de Distribuição ....... 37

Figura 11. Mapa de Localizaçăo da Bacia Experimental de Cunha .................... 47

Figura 12. Mapa planoaltimetrico da Bacia experimental "D" ........................

Figura 13. Relaçăo entre a precipitaçăo e o escoamento mensal ...................... 51

Figura 14. Variáveis utilizadas no modelo de simulação .................... 56

Figura 15. Representação da caracterização das hidrografas apos ajuste pelo metodo dos percentis .................... 57

Figura 15. Representação da caracterização das hidrografas apos ajuste pelo metodo das alturas de lamina d'água ............58 
viii

Figura 17. Teste do modelo proposto .............61

Figura 18. Resumo da metodologia para MSHJ e MSHA ..... G2

Figura 19. Distribuição de Freqüência da al turas de lámina d'água das chuvas atuais da Bacia Experimental "D", núcleo de Cunha S.P...... 65

Figura 20. Distribuiçßes das chuvas de altura minima da Bacia de Cunha S.P. ............... B7

Figura 21. Distribuiçōes das chuvas de altura modal da Bacia de Cunha S.P. ............... 68

Figura 2e. Distribuições das chuvas de altura media da Bacia de Cunha S.P. ............... 89

Figura 23. Distribuiçôes das chuvas de altura máxima da Bacia de Cunha S.P. ..............70

Figura 24. Distribuiçăo de Frequência da alturas de lamina d'água das vazóos das hidrógrafas da Bacia Experimental "D", núcleo de Cunha S.P.. 72

Figura 25. Distribuiçర̃es das vazôes minimas das hidrografas na Bacia de Cunha S.P. .......73

Figura 26. Distribuiçб̋es das vazôes modais das hidrografas na Bacia de Cunha S.P. ....... 74

Figura 27. Distribuiçốs das vazôes medias das hidrografas na Bacia de Cunha S.P. ....... 75

Figura 28. Distribuiçớes das vazb̂es máximas das hidrografas na Bacia de Cunha S.P. ....... 76

Figura 29. Método de classificação de hidrografas distáncia "Ra" ................... 93

Figura 30. Simulaçช̋es de hidrografas de classe $70 \mathrm{~cm}$ de vazão com $R a=0.62$ e $R a=0.91$ respecti vamente ................. 96

Figura 31. Simulaçóes de hidrografas de classe $70 \mathrm{~cm}$ de vazão com $R a=0.65$ e $R a=0.71$ respectivamente ................... 97 
Figura 32. Simulaçరes de hidrografas de classe $70 \mathrm{~cm}$ de vazão com $\mathrm{Ra}=0.73$ e $\mathrm{Ra}=0.53$ respecti vamente ..................

Figura 33. Simulaçßes de hidrógrafas de classe $70 \mathrm{~cm}$ de vazão com $R a=0.80$ e $R a=1.72$ respectivamente .................. 99

Figura 34.Simulaçôes de hidrógrafas de classe $70 \mathrm{~cm}$ de vazão com $\mathrm{Ra}=0.95$ e $\mathrm{Ra}=1.04$ respectivamente .................. 100

Figura 35. Simulaçớes de hidrografas de classe $70 \mathrm{~cm}$ de vazão com $R a=1.38$ e $R a=1.61$ respectivamente ................... 101

Figura 36. Simulaç̧es de hidrografas de classe $70 \mathrm{~cm}$ de vazăo com $R a=1.72$ e $R a=1.44$ respecti vamente .................. 102

Figura 37 . Simulaçßes de hidrografas de classe $70 \mathrm{~cm}$ de vazão com $R a=2.47$ respecti vamente . . . . . 103

Figura 38. Stmulaç̧̋es de hidrógrafas de classe $140 \mathrm{~cm}$ de vazão com $\mathrm{Ra}=1.34$ e $\mathrm{Ra}=1.44$ respectivamente ................. 104

Figura 39. Simulaçóes de hidrógrafas de classe $140 \mathrm{~cm}$ de vazão com $R a=1.51$ e $R a=3.14$ respectivamente .................. 105

Figura 40. Simulaçôes de hidrógrafas de classe $140 \mathrm{~cm}$ de vazão com $R a=2.01$ respecti vamente ...... 108

Figura 41. Simulaçóes de hidrografas de classe $140 \mathrm{~cm}$ de vazão com $\mathrm{Ra}=2.07$ e $\mathrm{Ra}=1$. 6e respectivamente .................. 107

Figura 42. Simulaçbes de hidrografas de classe $140 \mathrm{~cm}$ de vazão com $\mathrm{Ra}=6.31$ respectivamente ....................108 
Figura 43. Simulaç̧̃es de hidrógrafas de classe $210 \mathrm{~cm}$ de vazão com $\mathrm{Ra}=2.80$ e $\mathrm{Ra}=3.1 \mathrm{z}$ respecti vamente .................. 109

Figura 44. Simulações de hidrógrafas de classe $210 \mathrm{~cm}$ de vazao com $\mathrm{Ra}=3.45$ e $\mathrm{Ra}=3.60$ respecti vamente .................... 110

Figura 45. SimulaçBos de hidrógrafas de classe $210 \mathrm{~cm}$ de vazao com $\mathrm{Ra}_{2}=2.24$ e $\mathrm{Ra}=5.47$ respecti vamente ................... 111

Figura 46. Simulaçôes de hidrografas de classe $280 \mathrm{~cm}$ de vazão com $R a=4.23$ e $R a=4.77$ respecti vamente ................... 112

Figura 47. Simulaçß̋es de hidrografas de classe $280 \mathrm{~cm}$ de vazão com $\mathrm{Ra}=4.718$ e $\mathrm{Ra}=7.70$ respect 1 vamente ................... 113

Figura 48. Simulaçðes de hidrografas de classe $280 \mathrm{~cm}$ de vazão com $R a=8.50$ respecti vamente .................... 114

Figura 49. Simulaçóes de hidrografas de classe $350 \mathrm{~cm}$ de vazão com $\mathrm{Ra}=6.47$ e $\mathrm{Ra}=6.98$ respecti vamente ................... 115

Figura 50. Simulaçós de hidrografas de classe $490 \mathrm{~cm}$ de vazão com $\mathrm{Ra}=15.12$ respecti vamente ...... 116 


\section{LISTA DE TABELAS}

Tabela 1. Alguns projetos e estudos de bacias hidrograficas ................... 11

Tabela 2. Aplicaçỡes dos Sistemas de Transiação . . . . . 44

Tabela 3. Caracteristicas fisicas da Bacia Experimental "D" ................. 48

Tabela'4. Precipitação, altura de lámina d'água e vazão mensal, máxima e minima da Bacia Experimental "D" ...................5 52

Tabela 5. Identificação dos eventos chuvosos . . . . . . . 54

Tabela 6. Variaveis consideradas em cada evento churoso ......................... 54

Tabela 7. Estatisticas da distribuição das intensidades $(\mathrm{mm} / \mathrm{h})$ das chuvas atuais ............65

Tabela 8. Estatisticas da distribuição das intensidades de vazão das hidrógrafas model adas ....... 72

Tabela 9. Momentos das variáveis da chuva - CHUVAR ... 80

Tabela 10. Quartis das variaveis da chuva - CHUVAR .... 81

Tabela 11. Quartis das variaveis da hidrografa HIDVAR ................... 83

Tabela 12. Momentos das vartáveis da hidrógrafa HIDVAR .................... 83

Tabela 13. Distribuição de Frequencia do volume das hidrografas .................... 
$x i i$

\title{
MODELAGEM E SIMULACÃO DE HIDRÓGRAFAS
}

\author{
Autor : JOSE RAIMUNDO DE SOUZA PASSOS \\ Orientador : Prof. Dr. WALTER DE PAULA LIMA
}

RESUMO

Este estudo apresenta dois metodos de model agem e simulação de hidrógrafas em microbacias: MSHJ Modelos de Similação com base no Sistema Johrison de distributção de probabilidade, e MSHA - Modelos de Simulação com base nas Alturas de lamina d'água, a partir de padröes de precipitaçăo.

Para: tal foram isoladas 82 hidrografas acompanhadas das respectl vas chuvas anteriores (máximo de seis), num periodo de 18 meses (ABR-DEZ 83), na Bacia Experimental "D" do Núcleo de Cunha, pertencente ao Instituto Florestal de Săo Paulo.

As variáveis consideradas em cada evento chuvoso foram : percentis $625,50,75 \approx 100 \%$ da distribuição de volume de chuva no tempo, o volume da chuva, e as diferenças de tempo entre a chuva mais recente Cchuva zero - chos a as cinco últimas chuvas.

As variaveis de localização das hidrógrafas, inicio e fim do escoamento $l_{e} e \lambda$ respectivamentes, 0 volume total escoado $\mathrm{CV}$, foram corrrelacionadas com as caracteristicas das chuvas.

Ja as variáveis ligadas a forma da hidrografa diferiram segundo o metodo utilizado:

MSHJ : Tais modelos săo caracterizados por correlacionarem os dois parametros $(\gamma, \delta)$ do Sistema Johnson de Distribuiça de Probabilidade, com as variáveis das 
chuvas.

MSHA : Tais modelos são caracterizados por correlacionarem as alturas de lamina d'água, em intervalos de tempo de 20 minutos, com as variáveis das chuvas, simulação ponto a ponto da hidrógrafa.

Para a simulação foram utilizadas as proprias chuvas que alimentaram os modelos, possiblittando-se verificar a qualidade de ajuste para cada uma das 82 situaçóes, dentro de cada método, como também comparar os dois modelos propostos.

Para comparar a qualidade de simulação das hidrografas, foi desenvolvido um metodo denominado discriminantes de ajustes "Ra". Este tem como base a média - o desvio padrão dos desvios absolutos das distribuiçס̃es observada e esperada.

O inicio e fim das hidrografas $C \lambda$ e parametros de localizaçăo, eståo fortemente correlacionados somente com o volume e o padrão da chuva mais recente (chos.

O volume da hidrografa $f$ influenciado pelas duas churas mais proximas, cho e ch1s, atraves das varláveis volume e diferença de tempo entre duas chuvas (tcho1).

O modelo MSHJ produziu hidrografas com formas totalmente diferentes das hidrografas observadas, sendo então considerado um modelo năo adequado para o propostto. A baixa sensibilidade dos parâmetros de forma do Sistema Johnson de Distribuiça de Probabilidade $C \&$ e $\gamma$, fol a principal causa da qualidade das simulaçôs.

o modelo MSHA produziu em sua maioria hidrografas com formas semelhantes as hidrografas observadas. Das 34 equaçós de simulação das alturas de 
$x i v$

lamina d'água, a variável volume da chuva mais proxima (volcho) foi a mais frequiente $(87.5 \%$, e percentil 50 da chuva zero (p50cho) com frequiencia de $25 \%$.

O método das distáncias "Ra" se mostrou adequado para a função de "medir" ajustamentos de hidrografas, sendo possivel ser utilizado para classificar e selecionar ajustamentos de quaisquer outras variáveis. 


\title{
HYDROGRAPH MODELLING AND SIMULATION
}

\author{
Author : JOSE RAIMUNDO DE SOUZA PASSOS \\ Adviser : Prof. Dr. WALTER DE PAULA LIMA
}

\section{SUMMARY}

This study presents two methods of modeling and simulation of catchments hydrographs: MSHJ : Hydrograph Simulation Model based on the Johnson's System of Probability Distributions, and MSHA : Hydrograph Simulation Model based on the streamflow stage from precipitation patterns.

The data base comprised 82 stormflow hydrogaphs and the respective antecedent precipitation data Cmaximum of six, for a 18 months period (April-December /83), from the experimental catchment " $D$ ", of the "Núcleo Cunha , Instituto Florestal de São Paulo".

For each rainfall event, the variables used were : percentiles $625,50,75 \in 1005$ of the distribution of rain volume with time, rain volume and the time differences between the most recent rainfall czero rainfall), and the last five rainfall events.

The hydrograph localization, beginning and end of storm runoff $C \&$ and $\lambda$ respectively $)$ and total stormflow volume CV, were correlated with the precipitation patterns.

The variables associated with the shape of hydrograph, on the other hand, differed according to the method used:

MSHJ : Such models are caracterized by correlating the two 
Johnson's System of Probability Distributions parameters $(\gamma, \delta)$, with the rainfall variables.

MSHA : Such models are carcterized by correlating streamflow stage data, in $20 \mathrm{~min}$ time intervals, with the rainfall variables.

The simulation was carried out with the same precipitation, which were used in the modeling, thus permitting data a check in the fitness for each one of the 82 events, within each studied method, as well as comparing the two proposed methods.

The fitness of the hydrograph simulation was based on the development of a criterion called fitness discriminant "Ra". This is calculated based on the mean and the standard deviation of the absolute deviations of the expected and observed distributions.

The beginning and end of the expected hydrographs $(\lambda, \varepsilon)$, localizations parameters, are strongly correlated only with the volume and pattern of the most recent rainfall (CHO).

The hydrograph volume is influenced by the two most recent rains $\mathrm{CCHO}$ and $\mathrm{CH}$ I), through the variables volume and time difference between this two rainfall (TCHO1).

In general, the MSHJ model produce hydrographs with shape very different from the observed hydrographs. It is supposed that this was a result of the low sensibility of the shape parameters of the Johnson System $(\delta, \gamma)$.

The MSHA model produce, in general, hydrographs with shape similar to the observed hydrographs. The variable volume of most recent rain (Volchos was the most frequent $(87.5 \%$ within the 34 simulation equations, followed by the variable p5ocho cpercentile 50 of the zero 
xvii

rain $625.0 \%$

The fitness discriminant method (Ra) was

found to be adequate for the measurements of the adjustment of the hydrographs. It is, therefore a method which is suitable for the classification and selection of adjustments of any other variables. 
1. INTRODUCÃ̃ :

"O místico cré num deus desconhecido.

"O pensador e cientista crem numa ordem desconhecida.

"E diflcil dizer qual deles sobrepuja - outro en sua devoça não racional.

(L. L. WHYTE)

1.1. Caracterização do problema

1.2. Objeti vos 


\subsection{Caracterização do problema}

- manejo de bacias hidrograficas tem como objetivo a administração dos recursos naturais de uma dada bacia (sistema) de maneira racional, visando a produção de agua em qualidade e quantidade, utilizando resultados experimentais da hidrologia florestal.

o conhecimento da hidrologia de determinada bacia fundamental para que possamos entender a dinamica da agua no sistema sua relação com variáveis ligadas a fatores climáticos, silviculturais e fisiograficos. Variaveis estas cujas complexas interaçơes são refletidas na produção de água de uma dada bacia hidrografica, quantificada atraves da variação da vazão no tempo hidrografa.

- mecanismo de geração da hidrógrafa, após determinada chuva numa bacia hidrografica, vem já há alguns anos sendo foco de estudos. Apesar da existéncia de teorias numa tentativa de explicação deste mecanismo, só recentemente após o advento da informática e juntamente com métodos fisicos e estatisticos, \& que foi possivel uma melhor compreensão do fenómeno, direcionando-se entao os estudos no sentido de utilização de modelos de simulação.

Entende-se por modelo a representação de um sistema atraves de alguma forma que não a original sendo um sistema um grupo de objetos ou fatos unidos por alguma forma de interação ou interdependencia, executando funçð̋es especificas.

Deste modo, estudos que visam o entendi mento do mecanismo de geração da hidrógrafa, devem ser uma das prioridades em ecossistemas tropicais a subtropicais, principalmente em regióes com estação seca definida, periodo no qual a produção de água está estritamente dependente do periodo chuvoso anterior, quando da "recarga" 
da bacia, através do mecanismo da precipitação e consequiente infiltração da agua no solo.

No Brasil os ecossistemas de floresta tropical e subtropical vêm sendo degradados já há vários anos. Dentro deste contexto, a Mata Atlantica \& atualmente reflexo deste processo de degradação ao longo da costa brasileira. Atualmente, após a criação do Laboratório de Hidrologia Florestal no Parque Estadual da Serra do Mar no Estado de Săo Paulo, e juntamente com a cooperaçăo de orgãos de pesquisa e outras entidades, está sendo possivel melhor compreender este complexo e importante ecossistema.

\subsection{Objetivos}

Este trabalho tem com objetivo a elaboração de um modelo para simulação de hidrógrafas numa dada bacia hidrografica.

Para atingir esta meta, as seguintes etapas foram necessárias :

a) obtenção de dados de distribuição de vazão no tempo e as respectivas distribuiços de chuvas, ocorridas num periodo máximo de até seis dias após o evento da hidrografa;

b) caracterização das distribuiçỡes de precipi tação atraves de determinadas estatisticas;

c) ajuste das hidrografas utilizando-se o Sistema de Johnson de Distribuicão de Probabilidade;

d) elaboração de dois tipos de modelos de regressão múltipla :

13 correlação entre os parâmetros da etapa Cl com a etapa b);

2) correlação entre as alturas de vazão obtidas em aj,com os parametros da etapa b) ;

e) validação dos modelos. 


\title{
2. REVISÃO BIBLIOGRAFICA
}

\author{
"Toda ciencia seria superflua \\ se a aparencia, a forma das \\ coisas fosse totalmente \\ identica a sua natureza". \\ (Kar 2 Marx).
}

As consideraçбes relativas a este capitulo são importantes, na medida em que e possivel se ter inicialmente uma visão do conteúdo cientifico relacionado ao tema posteriormente, após o capitulo referente ao método utilizado, obter um posicionamento do escopo deste trabalho em relação ao conteúdo cientifico anteriormente citado. A estrutura da revisão obedeceu a seguinte sequéncia:

2.1. Ciencia e hidrologia

2.2. Bacias hidrográficas

2. 3. A hidrógrafa

2. 4. A geração do deflúvio e seus mecanismos

2.5. Modelos hidrológicos - classificação escolha 


\subsection{Ciéncia e Hidrologia}

De forma organizada a ciencia busca a ordem - conhecimento das coisas, busca algo invisivel, um mecanismo desconhecido que não se pode atingir. Diante desde contexto os fatos se apresentam para o cientista como algo de concreto e paupável, e juntamente com o uso da imaginação, permitem lançar hipoteses a respeito do funcionamento do mecanismo, obtendo-se então os modelos ou também denominado de construçß̋es de imaginação por ALVES (1986).

$$
\text { Atualmente a hidrologia vem sendo }
$$
questionada com relação a sua atuação como ciéncia ou como tecnologia CKLEMES, 1983; DOOCE, 1986; KLEMES, 1986; KLEMES, 1988). A questão de escala e conceitualização em hidrologia enfocada como fator determinante no avanço ou não do processo cientifico (KLEMES, 1983 ).

DOOGE (1986) diferencia de modo esquemático a atuaça do pesquisador cientifico e do engenheiro representado na Figura 1 .

O cientista trabal ha na porção superior do diagrama, incluindo a observação e o entendimento; ja o engenheiro abrange o ramo inferior, incluindo a predição e - controle. Observa-se que o pesquisador cientifico tem como meta o entendimento, a compreensão do mecanismo, através da utilização de fatos e modelos, enquanto o engenheiro usa as observaçర̃es para chegar as prediçర̃es e aos controles, e posteriormente retornar com informacóes ao pesquisador cientifico sobre o funcionamento dos modelos propostos.

DOOGE (1986) acredita que a hidrologia necessita hoje de uma revolução cientifica similar à ocorrida na física no século XVII, relacionada a lei de Gravitação Uni versal de Newton. Evidentemente uma revolução cientifica não $\&$ um fenómeno isolado que pode ser 
determinado com exatidão; existe todo um contexto historico envol vido CKUHN, 1975 .

E fato que a hidrologia interage com varias outras áreas do conhecimento cientifico tais como Geologia, Engenharia Civil e Ciencias Florestais, fazendo com que seja utilizada por profissionais dessas áreas no sentido de solução de seus problemas especificos, atuando assim muito mais como tecnologia do que como ciencia, CKLEMES, 1986; KLEMES, 1988 .

O HOMEM E SEU MEIO AMBIENTE

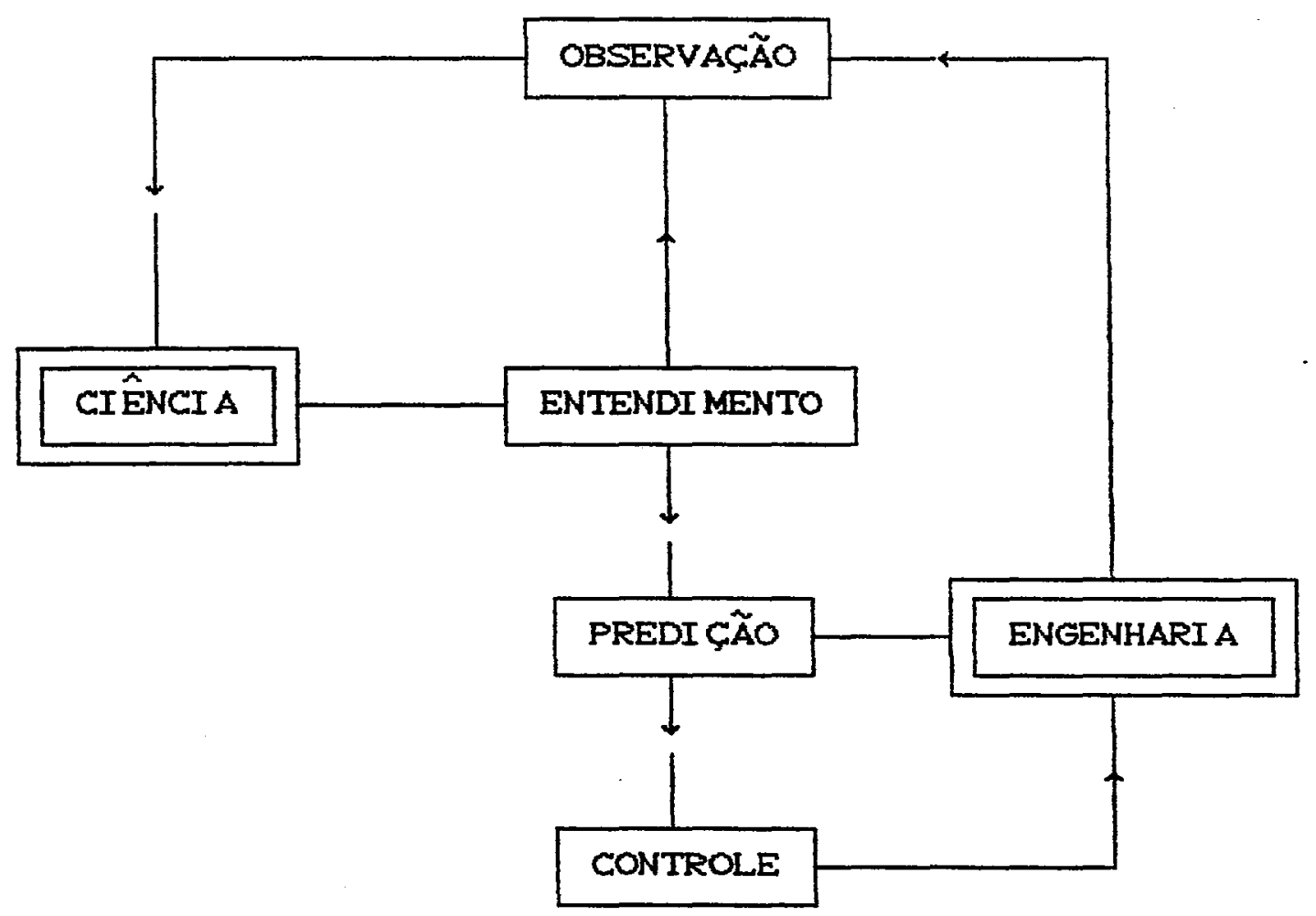

Figura 1 - Entendimento, prediçăo e controle CDOOGE, 19863. 
Por outro lado, e louvável a afirmação de DOOGE (1986), pelo fato de considerar a hidrologia como ciéncia da água.

Os modelos atualmente utilizados em hidrologia, principalmente aqueles relacionados à simulação das hidrógrafas para estudos relacionados às cheias, vêm sendo responsabilizados por contribuirem para que a hidrologia atue muito mais como tecnologia do que como ciencia. Existem tambem inúmeras criticas feitas com relação a parametrização exessiva e pouco conteúdo com fundamentos hidrologicos CDOOGE, 1986; KLEMES, 1986; KLEMES, 19883 .

\subsection{Bacias hidrográficas}

Para que se possa elaborar modelos em hidrologia florestal se torna necessário inicialmente conhecer as relaçర̋es entre a floresta e a água. Foi então definido um universo de estudo, uma unidade hidrológica, denominada bacia hidrográfica, CLEE, 1980 . LIMA (1986) define bacia hidrografica como : "toda área que proporciona o escoamento superficial para o canal principal e seus tributários".

Uma bacia e alimentada pela precipitação (P) em forma de chuva ou neve, e pela energia radiante (ER), a responsável pelo funcionamento do ciclo hidrológico. Esta bacia hidrográfica responde então com a produção de água através do deflúvio $(D)$, com a evapotranspiração $(E T)$ e com a variação de água no solo $C \Delta S$.

o principal objetivo da hidrologia é resolver a equação de bal anço hidrico (DOOGE, 1986 ):

$$
P-\mathbf{E T}-\mathbf{Q}-\Delta S=0
$$

A vazão CD, também denominada deflúvio ou escoamento total, tem como componentes: precipitação sobre 
os canais (PC), escoamento superficial ( $D_{s}$ ), escoamento subsuperficial (Qas), escoamento (ou fluxa) base (Qb), Cequação 2). Os elementos da equação (1) estão representados Figura 2 .

$$
Q_{t}=P_{c}+Q_{\theta}+Q_{8 s}+Q_{b}
$$

As microbacias hidrograficas se apresentam
como áreas de estudo mais apropriadas, como unidades
estruturais para estudo do ecossistema.

Sob o ponto de vista hidrológico o que caracteriza uma microbacia 0 fato de o componente escoamento superficial (D) atuar como fator dominante na geração do deflúvio em relação ao armazenamento sobre a rede de canais. Deste modo uma microbacia hidrografica muito sensivel a chuvas de alta intensidade courta duraçăos, como tambóm a fatores ligados ao uso da terra. Pode-se então definir uma microbacia hidrográfica como sendo aquela cuja area $\Leftarrow$ tão pequena, que a sensibilidade às chuvas de alta intensidade $e$ as diferenças de uso do solo, não é suprimida pelas caracteristicas da rede de drenagem; de acordo com tal definição, a área de uma microbacia pode variar de pouco menos de 1 ha ate cerca de 40 ha, podendo mesmo, em alguns casos, atingir a 100 ha ou mais (CHOW, 1964 ).

Assim, para microbacias o valor de $\mathrm{Pc}_{c}$ aproximadamente zero em ralação a $Q_{s}$; assim somando-se os componentes $Q_{3}$ e $Q_{s s}$ obtém-se o escoamento direto ou $Q_{d}$, equação (3).

$$
Q_{t}=Q_{d}+Q_{b}
$$




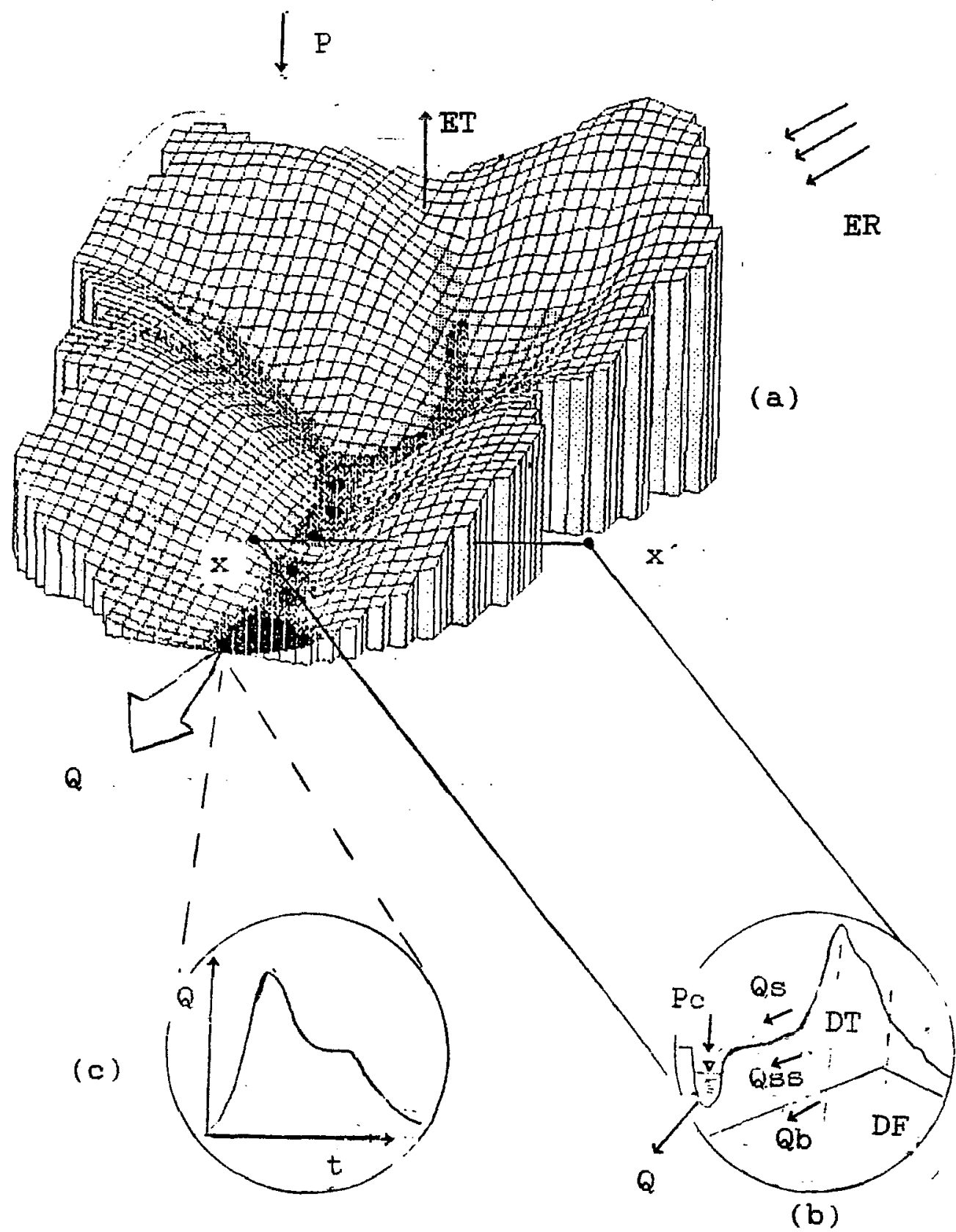

FIGURA 2 - Bacia Hidrografica e o seus componentes.

(a) Visão em perspectiva de uma bacia $\mathrm{XX}^{\prime}=$ secção; os elementos $\mathrm{P}, \mathrm{ET}, \mathrm{ER} \in \mathrm{Q}$

(b) Vista lateral com divisores topográficose freaticos CDT e DFJ; as componentes da vazão $P_{c}, Q_{s}, Q_{a s}$ e $Q_{b}$;

Cc3 Hidrografa (deflúvio $x$ tempo 3 . 
Uma microbacia pode ser considerada como um sistema que é alimentado por variáveis de entrada. respondendo com variáveis de saida, (Figura 3). A administração dos recursos de entrada é refletida na saida, dando a uma microbacia um carater funcional, com sensibilidade a modificações dos niveis de estimulos na entrada.

- manejo de bacias hidrogáficas tem interesse na saída ou resposta do sistema relacionado a produção de água, tanto sob o ponto de vista quantitativo como qualitativo.

RODDA (1985) faz um apanhado histórico dos estudos relacionados a microbacias hidrograficas resumido na Tabela 1. Observamos que tais estudos já eram conduzidos desde o seculo XVII.

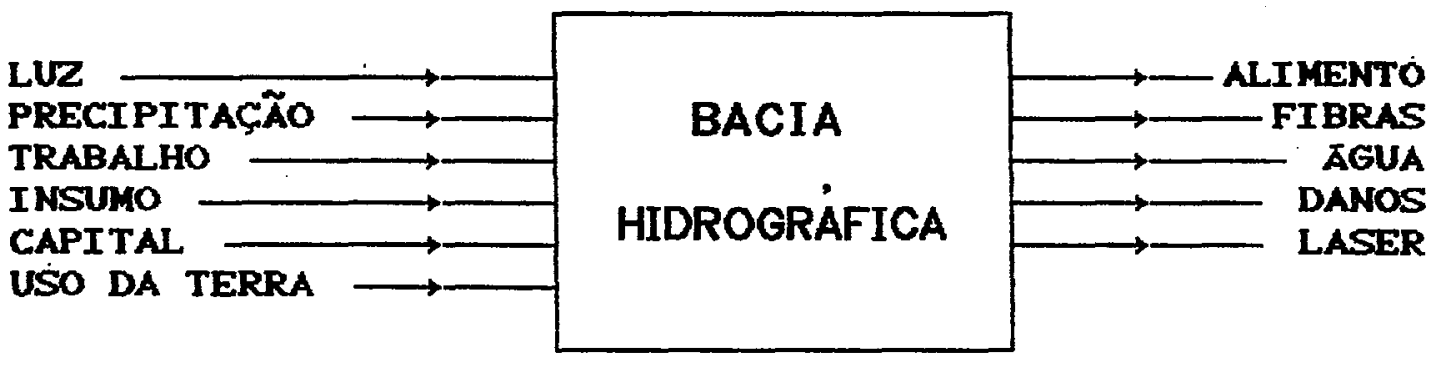

Figura 3 - Representacão de uma bacia hidrográfica como um sistema, mostrando as entradas ou estimulos e as saidas ou respostas.

No Brasil os estudos ligados a Hidrologia Florestal e a Manejo de Bacias Hidrográicas estão ligados a alguns orgã̃s tais como: Instituto florestal de sáo Paulo, Instituto Nacional de Pesquisas da Amazónia, CENA, EMBRAPA Universidades : ESALQ USP, UNESP, UFV O UFRJ. Destacam-se os trabalhos : (GICCO, 1885), (FRANKEN \& LEOPOLDO, 1984 ), CLEOPOLDO, 19823. 
Tabela 1- Alguns projetos e estudos de bacias hidrograficas segundo RODDAC 19853 .

\begin{tabular}{|c|c|c|c|c|}
\hline ANO & PAIS & BACIA & $\begin{array}{l}\text { AREA } \\
\left(\mathrm{km}^{2}\right)\end{array}$ & OBJETIVC \\
\hline 1674 & França & Aignayle le Duc & 121.5 & (1) \\
\hline 1686 & França & Here/Marriotte & 60.4 & (1) \\
\hline 1867 & Chekslovákia & - & - & - \\
\hline 1890 & Suiça & Sperbelgraben & 0.6 & (2) \\
\hline 1908 & Japã́o & Ota & - & \\
\hline 1910 & E. U. A. & Wagon Wheel Gap & 0.8 & (3) \\
\hline 1930 & U.R.S.S. & Valaday & - & \\
\hline 1930 & E. U. A. & San Grabriel Mountanis & - & $(4)$ \\
\hline 1932 & E. U.A. & Shackham Brook & 8.0 & $(5)$ \\
\hline 1934 & E.U.A. & Coweeta & 16.0 & (3) \\
\hline 1948 & Al emanha & Winteral & 0.8 & - \\
\hline 1950 & Africa & Ki makia & - & (3) \\
\hline 1950 & Reino Unido & - & - & \\
\hline 1953 & França & Al ance & 3.0 & $(7)$ \\
\hline 1957 & Africa & Sambret & 7.0 & (3) \\
\hline 1957 & Africa & Lagan & 5.5 & (3) \\
\hline 1988 & Canadá & Blue Springs Creek & 44.0 & cos \\
\hline 1970 & Nova Zelandia & - & - & (9) \\
\hline 1982 & Brasil - UNESP & $/$ INPA $/$ CENA & 1.3 & (2) \\
\hline
\end{tabular}

OBJETIVOS: (1) medição de deflúvio

(2) medição de deflúvio, precipitação, ET e balanço hídrico.

(3) balanço hidrico efeito de vegetação.

(4) efeito do fogo.

(5) práticas agrícolas poluição do ar.

(6) métodos de predição de deflúvio e qualidade da água.

(7) rel ação chuva-defl úvio.

(8) predição de deflúvio.

(9) conceito de área variável. 


\subsection{A Hidrografa}

Reescrevendo a equação (1) do balanço hidrico e levando em conta que :

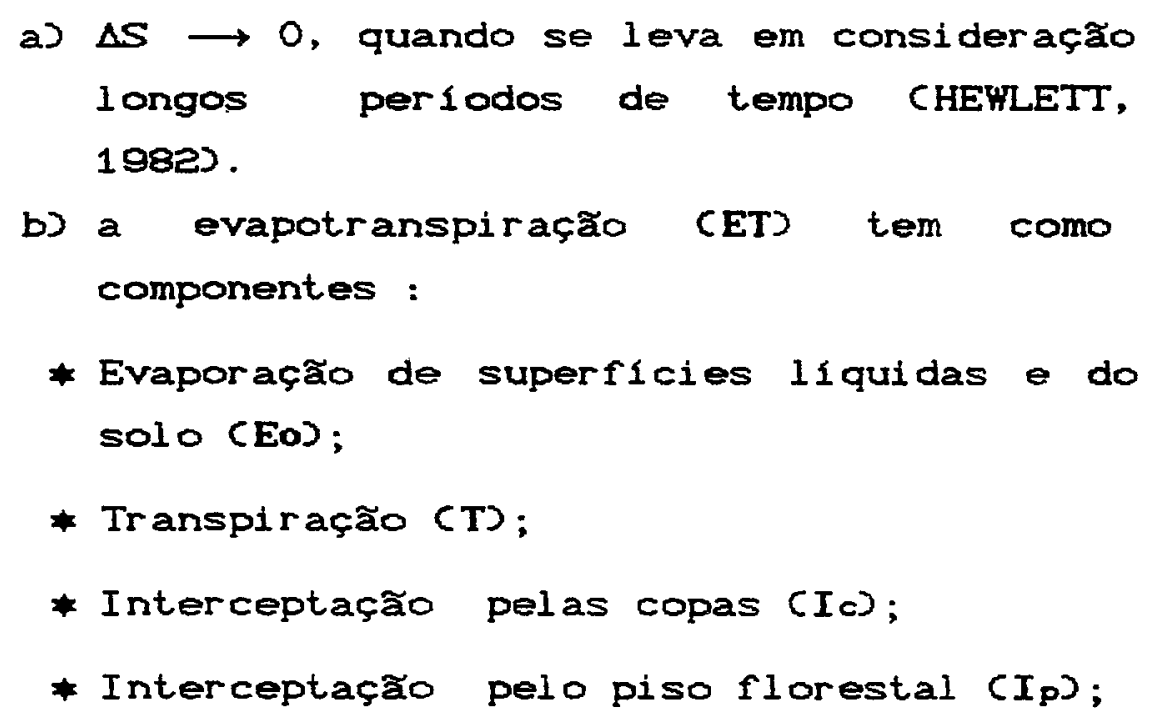

Deste modo pode-se escrever :

$$
\left.Q=P+C T+I_{c}+I_{P}+E_{0}\right)
$$

A expressão acima nos mostra que a vazão $C Q$ é resultado de interaçós das entidades que a comporem. Desta forma, o estudo das variaçós de $C D$ no tempo pressupỡe o conhecimento a a modelagem de seus componentes, principalmente $P$, Io e Eo CBERNDTSSON \& NIEMCZYNOWICZ, 1988; BEVEN, 1979; BRUIJNZEEL \& WIERSUM, 1987; CALDER et alii, 1986; CLARKE, 1986/87; FRANKEN et alii, 1982; GASH, 1979; HAAN \& JOHNSON, 1989; ISTOK \& BOERSMA, 1989; JACKSON, 1975; LAINE, 1969; LEONARD, 1967; LIMA, 1987; LLOYD et alii, 1988; OSBORN, 1984; RUTTER, et alii $1971 / 723$.

A Hidrografa como sendo o resultado da distribulçăo da vazão no tempo, está representada na Figura 
4. juntamente com os seus componentes e a distribuição da precipitação que deu origem ao escoamento.

Não existe uma expressão ou função matemática que relacione o deflúvio com o tempo, isto $e, Q t=f(t)$. A distribuição de Qt no tempo assume várias formas, sendo resul tado de complexas interaçóes.

Deve-se destacar que o padrão de precipitação tem responsabilidade exclusivamente sobre o ramo de ascenção do hidrograma, enquanto que as caracteristicas fisicas da microbacia hidrográfica influenciam o ramo de recessão do hidrograma, CLIMA, 19863.

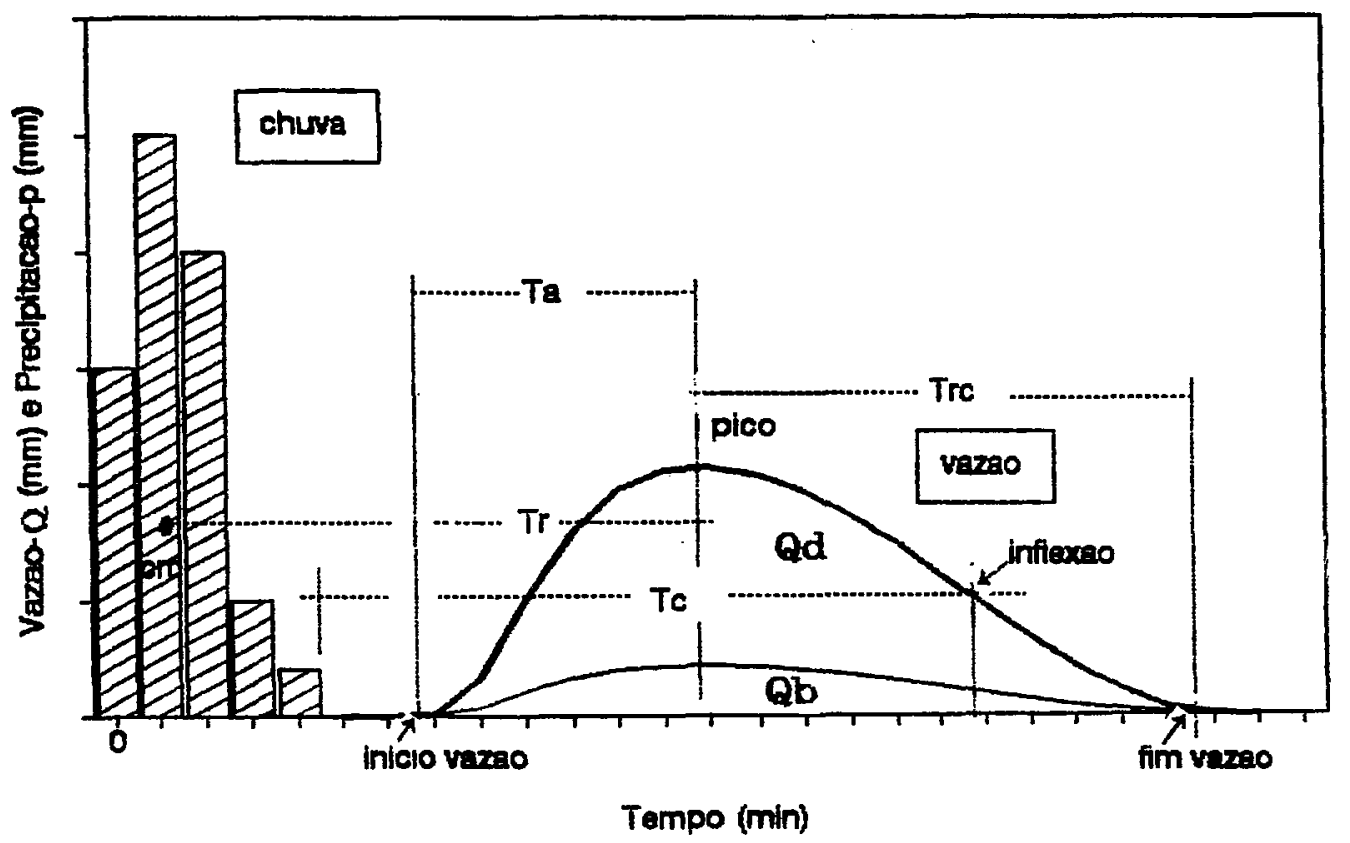

Figura 4 - Componentes da hidrógrafa. Tr=tempo de resposta; Tc=tempo de concentração; Trc=tempo de recessão; Qd=escoamento direto; $\mathrm{Ob}=e s c o a m e n t o$ de base; Ta=tempo de ascensão. 
Muitos estudos têm sido conduzidos no sentido de verificar o efeito do uso da terra e seu reflexo nas hidrografas em bacias hidrográficas.

Observaçôs realizadas em pequenas bacias mostram que fatores como urbanizaçăo, construçăo de terraços e estradas agricolas, corte da floresta e uso de fogo, tem como conseqúéncia o aumento do pico da hidrógrafa; enquanto os efeitos de solo geologia da bacia estão relacionados a curva de recessão da hidrografa CBEVEN \& WOOD, 1983; PILGRIM et ali1, 1982; ROBISON, 1986 ).

BEVEN \& WOOD (1983), em estudos da natureza dinâmica de áreas de contribuição de vazão, atraves de uma metodologia de fácil uso, demonstram que a predição de vazão e freqüencia de cheias pode ser muito sensivel a suposiçชes a respeito da natureza de áreas de contribuiçao de vazão.

OSUNKOYA et alii (1984), estudando respostas de hidrógrafas em microbacias de Floresta Tropical Equatorial Chuvosa na Nigeria, correlacionaram parametros de hidrógrafas, precipitaçăo e caracteristicas fisiograficas. Durante o periodo de um ano foram encontradas baixas vazôes e os coeficientes do escoamento anual variaram de 1 a $40 \%$ da chuva anual. Embora não tenha sido encontrada correpondência entre a distribuição temporal da hidrografa de qualquer bacia hidrográfica e a distribulção da chuva, os parámetros do escoamento se apresentaram altamente influenciados pela natureza geomorfologica da bacia hidrográfica em estudo.

wOOD et alii (1988) acreditam que para melhor entendimento dos fatores que afetam a hidrógrafa necessário considerar áreas elementares de contribuição de cada bacia - ou seja, áreas "elementares" ( $\left.30 \mathrm{~m}^{2}\right)$, na qual se possam representar inúmeras variáveis relacionadas ao solo, evapotranspiração, escoamento, topografia, dentre 
outras - como estrutura base para a modelagem em bacias hidrograficas.

WHEATER et alii (1982), estudando efeltos da chuva e características de bacias, em particular parametros relacionados à umidade do solo, na forma da hidrográfa unitária instantánea CHIUD e 55 eventos em oito bacias no Reino Unido, encontraram grande variação da resposta da HUI às mudanças dos padróes da chuva, sendo bastante influenciada pela condição de deficit de umidade antecedente.

HEWLETT \& BOSCH (1984/85), trabalhando com 1546 chuvas com precipitação inferior a $20 \mathrm{~mm}$ em oito pequenas bacias de 25 a 254ha na Africa do Sul, testaram atraves de regressão múltipla, possiveis efeitos em hidrógrafas. Foram utilizadas como variávels: precipitação incidente, intensidades máximas de precipitação durante o evento chuvoso a 30 e $60 \mathrm{~min}$, duração da chuva, e ate 3 chuvas antecedentes (diárias) e uma variável binária representando a estação do ano. Ficou demonstrado que a intensidade de chuva das oito bacias consideradas na media, contribuiu para explicar $5 \%$ da variação do deflúvio e aproximadamente $20 \%$ das variaçőes dos picos nas hidrografas.

ISTOK \& BOERSMA (1986) estudaram efeitos de chuvas de baixa intensidade $(\langle 15 \mathrm{~mm} / \mathrm{h})$ a ocorréncia de hidrografas em cinco microbacias (0.46-285 ha). As chuvas de $12,48,120$ horas precedentes a cada hidrógrafa, sob o ponto de vista quantitativo, não causaram escoamento em $44 \%$ a $93 \%$ dos eventos observados. O estudo mostra também que a magnitude da chuva durante 12 e 120 horas precedentes, foram mais significativas na determinação de ocorréncia da hidrografa do que na magnitude de chuvas de intensidade media ou maxima; que para climas caracterizados por baixa intensidade de precipitação, as chuvas antecedentes são 
mais importantes no controle da ocorrencia e quantidade do deflúvio do que a intensidade da chuva mais recente considerada.

Com relação ao efeito de padröes de

precipitação no escoamento superficial, destaca-se o trabalho desenvol vido por WOOLHISER \& GOODRICH (1988).

\section{4. A Geracão de deflúvio e seus mecanismos}

CHOW (1964) \& WISLER \& BRATHER (1964)

dividem os fatores que afetam a geração do deflúvio em dois grandes grupos: aqueles relacionados ao clima (principalmente a precipitação), e aqueles relacionados às caracteristicas fisicas de uma dada bacia hidrografica. CHOW (1964) considera os fatores climáticos como : tipo de precipitação; intensidade, duração e distribuição da chuva sobre a bacia; deslocamento e direção da chuva; precipitaçăo antecedente e umidade do solo, e outras condiçôes climáticas. Já as caracteristicas físicas consideradas são : utilização da terra, tipo de solo, área, forma, altitude, declividade, orientação, tipo de drenagem efetiva, extensão da drenagem indereta, drenagem artificial.

WISLER \& BRATHER (1964), adverte do perigo para quem tentar encontrar uma equaçăo que explique as variações de vazão, podendo incorrer em erros que podem variar de $100 \%$ a $1000 \%$, dependendo do número de variáveis incluidas no modelo. E logico que o ideal seria encontrar uma equação que englobasse todas as variáveis. No entanto, mesmo que esta fosse encontrada, provavelmente seria complexa sob o ponto de vista matemático, e com scluçós aproximadas utilizando-se de equaçőes diferenciais, certamente não lineares, ou até mesmo sem solução, mesmo com toda ferramenta computacional existente.

Para o estudo da vazão, duas linhas podem 
ser seguidas:

$1)$ relaciona-se simplesmente atraves de uma equação de regressão a precipitação o o escoamento total em termos absol utos.

2) estudos que levam em consideração a distribuição da vazão (as vezes da precipitaçăo) no tempo - hidrógrafa.

No que diz respeito a primeira limha, os estudos indicam baixa correlação entre a precipitação e a vazão anual, devido ao grande número de variáveis envolvidas no processo; entretanto quando se aumenta o número de variáveis explanatórias, tais como frequéncia do evento chuvoso, condiçбes de umidade antecedente e outras, a correlação aumenta (CHOW 1964 ).

As condiçós de umidade antecedente, por exemplo, não podem ser determinadas diretamente, mas sim atraves de indices, como por exemplo o indice de precipitação antecedente (IPA, definido por Buttler ${ }^{1}$, citado por CHOW (1964).

Dentro dessa linha de obtenção do deflúvio. existem modelos que tém como base equaçz̃es de regressão linear simples, como o modelo apresentado por ULLAH \& BABU (1970), que trabalharam com efeito de condiçóes antecedentes à hidrógrafa, e MELANEM \& LAUKKANOM (1980), abordando aspectos relacionados a hidrologia urbana.

DRIVER \& TROUTMAN (1989), em estudos de manejo e planejamento urbano nos Estados Unidos, el aboraram um modelo de regressão múltipla tendo como entrada varláveis físicas, climáticas e de uso da terra, e como variável dependente o volume da hidrógrafa total.

1 Butler, S.S. Enginnering hyarolosy. Frentice-Hall, Inc., Englewood Cliffs, N.J., 1957, p. 227-229. 
Outros estudos utilizam modelos de auto-regressão com modelos ARMA e modelos ARIMA, CCLARKE, 1984; HINO \& HASEBE, 1981 ; WEEKS \& BOUGHTON, 19873 FEDORA \& BESCHTA (1989) demonstram a importancia do efeito da precipitação anterior através da utilização do IPA no

deflúvio em um modelo de simul ação em bacias hidrográficas no estado de Oregon, Estados Unidos.

Várias são as expressões que buscam estimar - deflúvio produzido: a) fómulas empiricas ou b) fórmulas cinematicas (que levam em conta o movimento da água) (LENCASTRE \& FRANCO, 1984). Tais expressöes tem sua importáncia em áreas como a Hidráulica, Engenharia civil e Irrigação. No entanto, tais expressరes não dão uma idéia da dinámica do deflúvio, alem do fato de terem pouca flexibilidade no tocante a utilização em locais diferentes daqueles nos quais foram concebidas.

E finalmente os estudos que enfocam a segunda linha utilizam metodos como o de área -tempo, considerado como extensão do método racional e o da hidrografa unitária CSHAW, 1983; ESCOLA POLITECNICA DA UNI VERSIDADE DE SAO PAULO, 1983 .

Para elucidar o fenómeno de geração de deflúvio, houve então a necessidade de um maior conhecimento do fluxo de água no solo, através de estudos que incluam os componentes do escoamento total: Qs, ass a Qb, CANDO et alii 1983; HARR, 1977; HEMLETT, 1961; LUXMORE, 1983; MARQUES FILHO, 1982; O'LOUGHLIN, 1986; PESCHKE \& KUTILEK, 1982; SLOAN \& MOORE, 1984 ).

HEWLETT \& HIBBERT (1967), estudando hidrografas de bacias hidrograficas experimentais de áreas montanhosas florestadas, conceberam o conceito de "área variável de influéncia". Segundo este conceito, nem o 
escoamento direto nem o rluxo de base produzido uniformemente sobre a bacia, mas o deflúvio está sobre influencia de uma área de origem dinámica a qual sofre expansores e contraç̧es, dal a denominação de área variável de influéncia. A geração de deflưvio nestas condiçơes pode ocorrer por duas vias (LIMA, 1986):

a) escoamento superficial: normalmenete existe um retardadmento desde a chegada da chuva até o inficio deste processo, que e decorrente do tempo gasto para o preenchimento do armazenamento das depressరos do terreno e da interceptação, em geral da ordem de 10 minutos CO'LOUGHLIN, 1981 ).

b) escoamento sub-superficial : o reterdamento $f$ maior, em função de que depende, também, do tempo gasto para a percolação da água no solo até a camada saturada, onde começa a haver a movimentação lateral que define o interfluxo. Portanto $e$, também função da profundidade do solo e de sua condutividade hidráulica, sendo em geral, da ordem de 5 h.

Vários autores reforçam a importáncia de estudos a nível de campo para elucidar o fenómeno de geração de deflúvio e posterior utilização em modelagem de hidrografas CBONELL et alii, 1984; DUNNE, 1983; KIRBY, 1980; PEARCE et alii, 1986; SKLASH et alii, 1986; TSUKAMOTO \& OHTA, 1988 ).

Os mecanismos de geração de deflúvio são descritos por DUNNE (1983), PILGRIM (1983) e RUPRECHT \& SCHOFIELD (1989), (ver Figura 5 :

a) escoamento superficial de Horton (produção de as) e gerado quando a intensidade de infiltração da chuva excede a capacidade de infiltração do solo. Este processo ocorre mais frequentemente em regiós áridas e semi-áridas, onde a densidade de vegetação é baixa, e com baixas taxas de infiltração; e em áreas perturbadas, 
como campos cultivadas, estradas rurais e áreas pavimentadas;

b) o escoamento subsuperficial cass, contribui significativamente para o escoamento total, onde a condutividade hidráulica do solo e elevada devido a textura grosseira do soloe as aberturas no solo feitas pela sistema radicular da vegetaçăo. 0 ass pode atingir os cursos d'água através das nascentes perenes, ou pode ser dividido lateralmente por alguma camada com uma condutividade hidráulica menor do que a intensidade da chuva; zonas com topografia concava tendem a contribuir mais para a produção de ass do que outras áreas.

c) escoamento superficial (Qs), que ocorre por saturação das camadas superficias do solo, devido a elevaçăo do nivel do lençol freatico, ou por percolação lateral ou vertical acima de um impedimento horizontal no solo.

Estas hipótese são representadas na Figura 5.

2.5 Modelos hidrologicos - Classtficaçăo e escol ha

CHOW (1964) define modelos hidrológicos como "formulaçర̃es matemáticas para simular fenómenos hidrologicas naturais, considerados como processos cqualquer fensmeno que experimenta mudanças relacionadas ao tempo..." D. Desta forma, todo fenómeno hidrologico que varia com o tempo é denominado processo hidrologico. Em forma de esquema, a classificação de processos hidrologicos E dada na Figura 6 . 

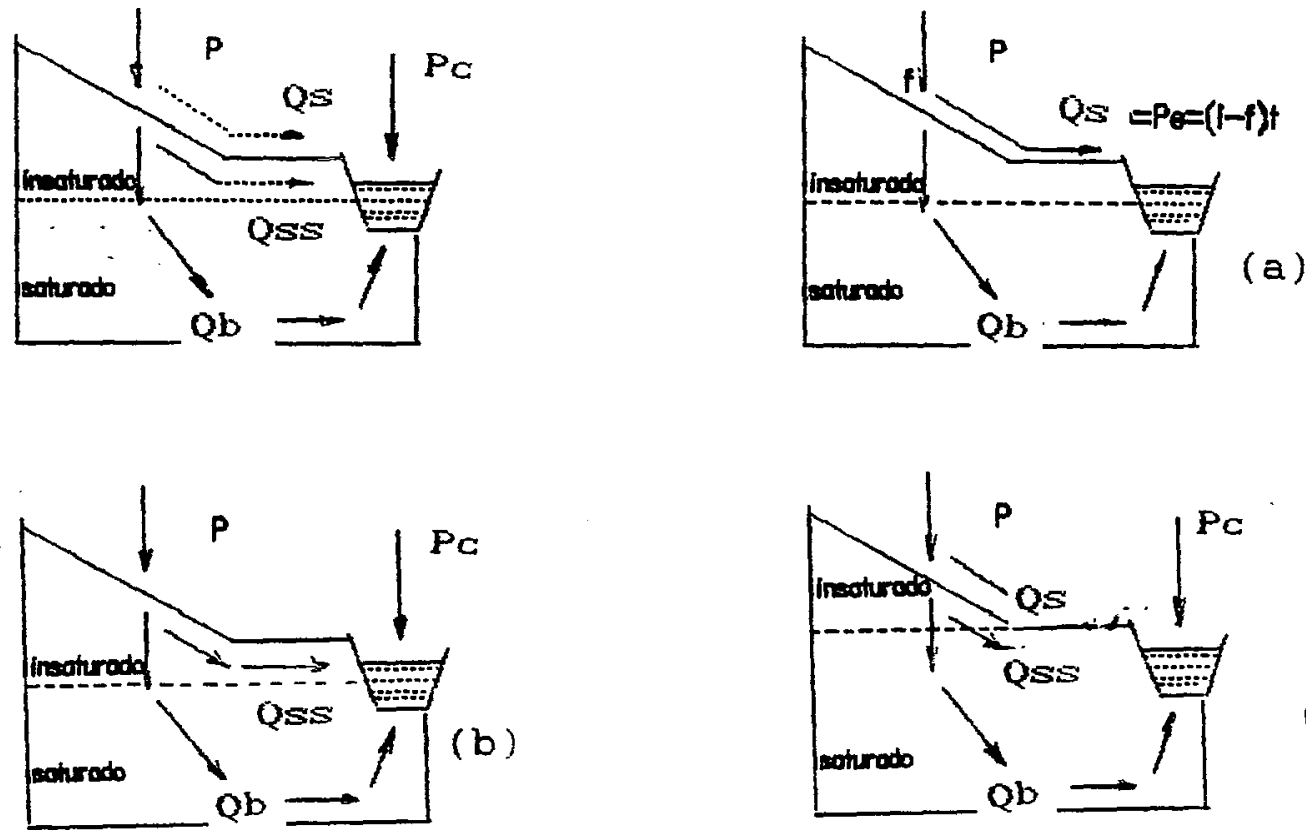

$$
\begin{aligned}
& \mathrm{P}=\text { precipitação direta } \mathrm{Pe}=\text { precipitação efetiva } \\
& \mathrm{Qs}=\text { fluxo superficial } \mathrm{Qss}=\text { fluxo subsuperficial } \\
& \mathrm{Qb}=\text { fluxo base } \quad \mathrm{i} \text { = intensidade da chuva } \\
& \mathrm{f}=\text { capacidade de infiltração de ácua no solo } \\
& t=\text { tempo }
\end{aligned}
$$

'Figura 5 - Mecanismos de geração do deflúvio. WARD, (1984a) 


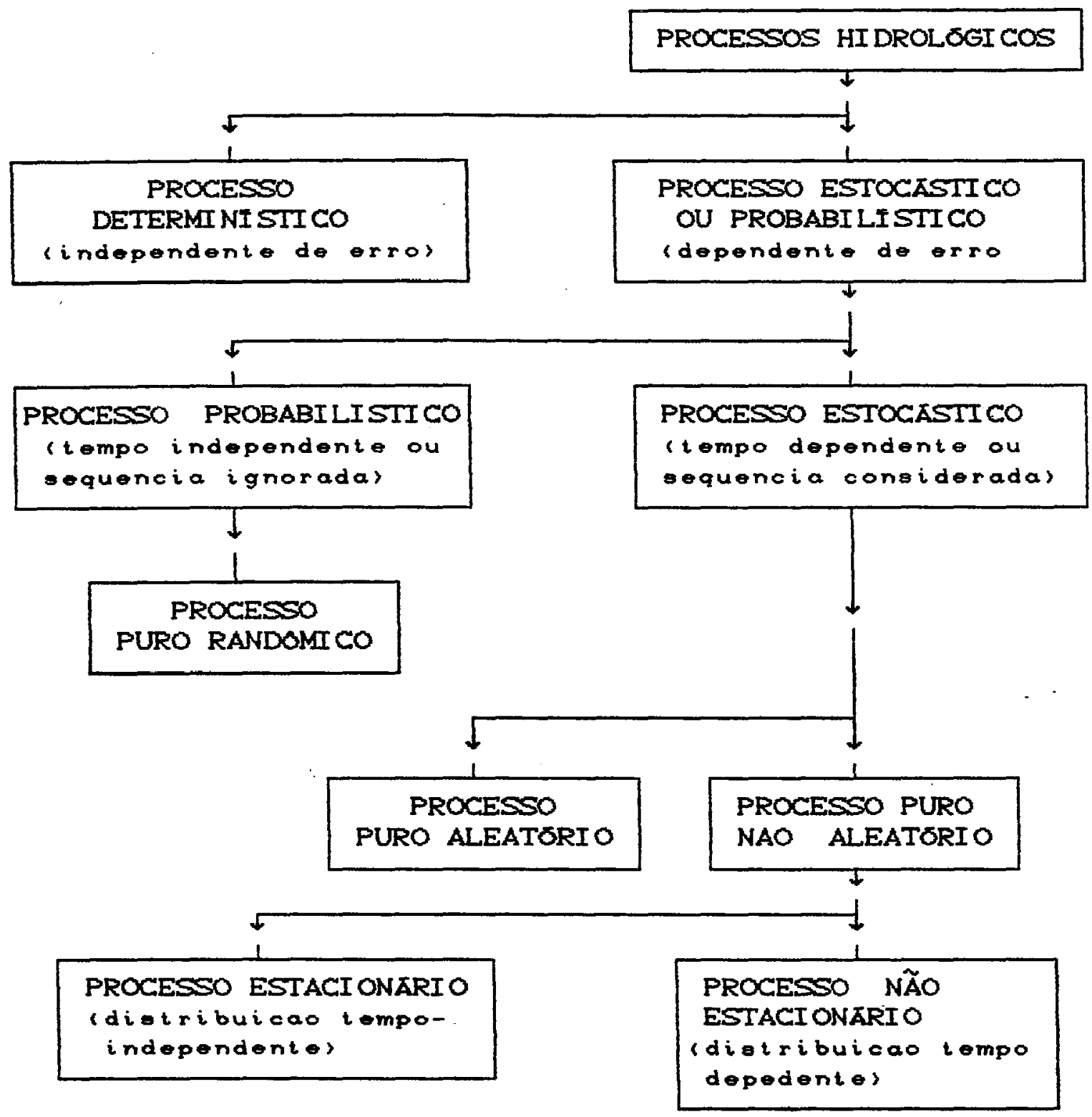

Figura 6 - Classificação dos processos hidrógicos segundo CHOW (1964), adaptado. 
CLARKE (1984) propõe um sistema de classificação similar ao de CHOW (1964), esquematizado na Figura 7 .

Cada compartimento o definido :

(i) ESTOCASTICO - Tanto $x(t)$ (variáveis de entrada) quanto $y(t)$ (variáveis de salda) e et (erro) têm distribuição de probabilidade, CHidrografa Unitária).

(ii) DETERMNISTICO - Todas as variáveis são livres com relação a variação aleatória; não têm distribuição de probabilidade, Cmodelos: Equação de Laplace para aquíferos, hidrógrafa unitária instántanea estimada via séries harmonicas).

(iii) CONCEITUAL - A função que correlaciona as variáveis elaborada levando-se em conta processos físicos.

CIV SISTEMA TEORICO-LINEAR CLST) - Noore sistema de superposição, isto é :

$x_{1}(t) \longrightarrow y_{1}(t)$
$x_{2}(t) \longrightarrow y_{2}(t)$$\Rightarrow \begin{aligned} & x_{1}(t)+x_{2}(t)= \\ & -y_{1}(t)+y_{2}(t)\end{aligned}$

(5).

(V) SISTEMA REGRESSAO LINEAR CLRS) : Se existir linearidade na relaçăo entre os parâmetros estimados.

(vi) " LUMPED " : Não leva em conta a distribuição espacial das variáveis de entrada $x(t)$.

(vii) DISTRIBUIÇAO DE PROBABILIDADE : Algumas variáveis de entrada têm distribuição de probabilidade assumida.

(viii) GEOMETRICAMENTE DISTRIBUIDO : Expressa a variabilidade em termos da rede de pontos (na bacia), relacionando suas distáncias. 


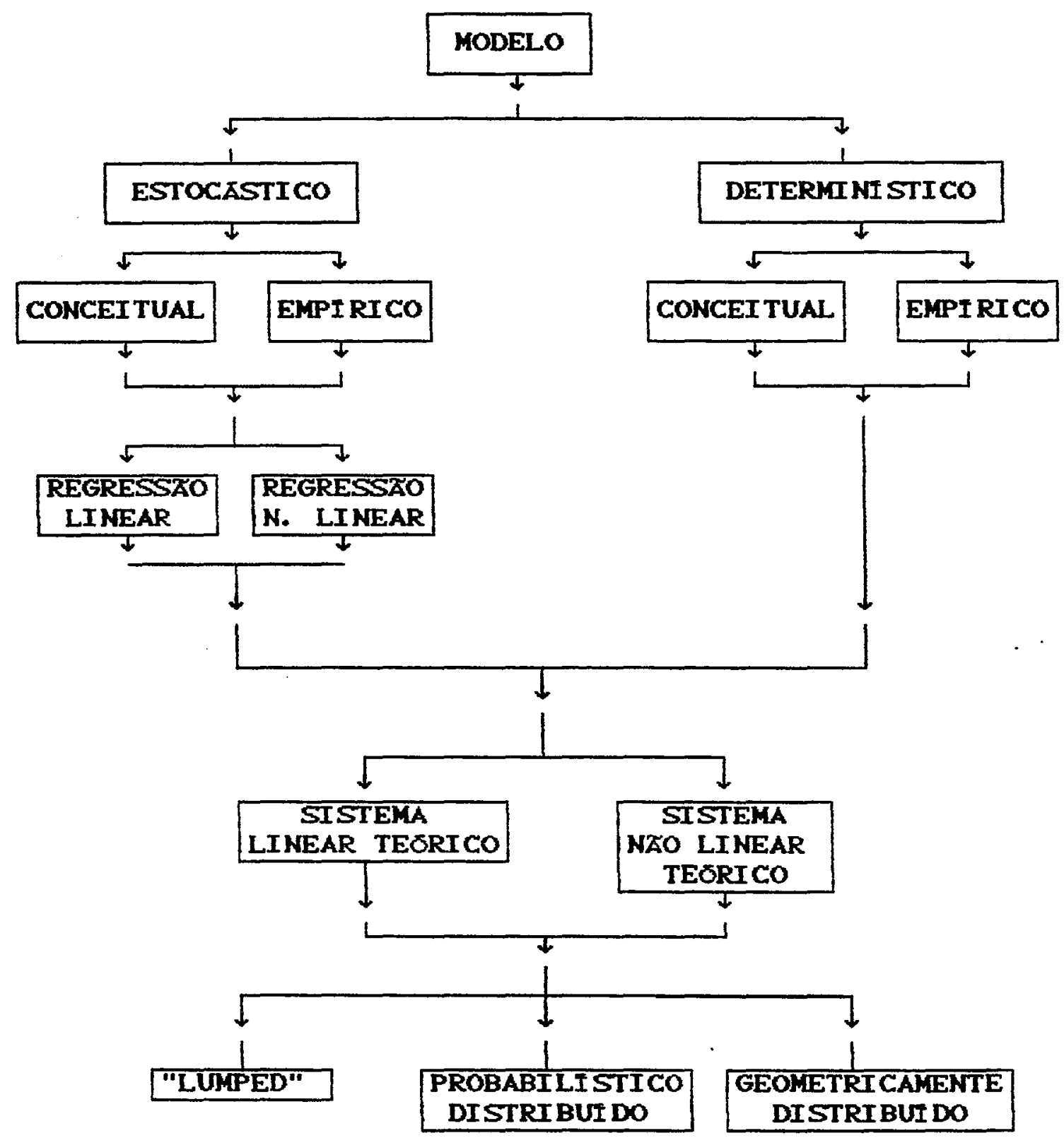

Figura 7-Esquema de classificação de modelos hidrologicos proposto por CLARKE (1984). 


\section{WOOLHISER \& BRAKENSIEK (1982) *}

Ozga-Zielinska ${ }^{1}$ apresentam criterios de classificação diferentes do apresentado por CLARK (1984).

Nesta última etapa, após a abordagem de classificação de modelos hidrológicos, serão enfocados alguns aspectos ligados à escol ha do modelo.

\section{WOOLHISER \& BRAKENSIEK (1982) apresentam}

alguns passos que podem ser seguidos na escol ha de modelos hidrologicos :

$1)$ Definir explicitamente o problema e especificar a informação requerida para al cançar os objetivos,

2) identificar' os modelos disponiveis,

3) especificar os criterios e limites que serão usados e definir os valores para cada modelo disponivel,

$4)$ estimar custos por itens para cada modelo,

$5)$ avaliar os modelos candidatos e fazer a selecão.

WOOD \& O'CONNELL (1985) propřem a distinção entre tres tipos de modelos: modelos distribuidos fisicamente, modelos conceitual "Lumped" e "caixa preta", também comertado em ANDERSON \& BURT (1985).

a) Distribuidos com base física : CABBOTT et alii, 1986a; ABBOTT et alii, 1986b; BARTEM \& BROOKS, 1985; BATHURST, 1986a; BATHURST, 1986b; BORAH, 1989; HAGER, 1984J. Sao utilizadas equaçóes de massa, energia e momentos para descrever o movimento da água sobre a superficie da terra e através de regióes saturadas e insaturadas. O sistema resultante de equações diferenciais tem que ser resolvido numericamente em todos os pontos na grade de representação do sistema bacia hidrografica $C$ WOOD \& O'CONNELL, 1985 .

\footnotetext{
1

OZGA-ZIELINSKA, MARIA Structure and operator functions of mathematical models of hydrology systems. $J$. Hydrolo8y, 3(1/2): 1-20, 1976.
} 
Em defesa dos modelos distribuídos, BEVEN

(1985) comenta que estes requerem grande investimento sob o ponto de vista da informática e programação, preparação de dados, e experimentação de campo.

BEVEN \& O'CONELL 2 citado por BEVEN (1985) identifica quatro grandes áreas que oferecem grande potencial para a aplicação dos modelos distribuidos: previsão de efeitos de uso da terra; previsão de efeitos da localização espacial das variáveis de entrada e saida; previsão do movimento de poluentes e sedimentos; e previsão de resposta hidrológica não monitoradas onde os dados estão disponi veis para os modelos " 1 umped".

Com modelos de base fisica e possivel extrapolar resultados para outros locais em outros periodos de tempo (BEVEN, 1985), tendo como elo a estimativa dos parametros (WILLIANS \& YEH, 1983). No entanto atualmente alguns pesquisadores questionam a sua aplicação devido a complexidade das equaçóes, sendo possivel que se perca os limites do processo e suas aplicaçães práticas CFERREIRA \& SMI TH, 19883 .

As questőes de limitação dos modelos com base física são firmemente questionadas por BEVEN (1989), Este aborda consideraçőes relacionadas a realidade heterogenea da bacia hidrográfica e dimensionalidade dos parámetros na calibração; HAAN (1988) comenta a respeito da incerteza dos parámetros. questóes de escala quando da transferéncia por modelos, e sugere que os modelos com base fisica atuais sejam utilizados como conceituais "lumped".

Diante de tantas mudanças nas ideias relacionadas à modelagem hidrologicas, os modelos estocáticos tém papel relevente para o futuro, CFREEZE, 1980; TAKASAO \& TAKARA, 1988 ; TODINI, 1988 .

BEVEN \& O'CONELL On the role of distributed models In hydrology. Institute of hydrolo8y, Report 81 , Wali ingford, UK, 1982. 
b) Modelos "Lumped" : BLACKIE \& EELES (1985) acreditam que a fase terrestre do ciclo hidrologico e um processo deterministico, que é obrigação dos hidrologistas procurar entende -los e quantifica-los.

O concel to de modelo "l umped" tende a ser considerado somente para pequenas bacias com vegetaçăo, geologia solos homogéneos, mas a experiencia tem mostrado que estes podem ser aplicados a uma ampla faixa de tamanhos de bacia com cobertura de vegetação, geologia e solos. Elevados padróes assimetricos de chuva também podem ser tolerados, estabelecidos que estes modelos são razoavelmente estáveis. O fator chave para o sucesso de modelos "lumped" e a estabilidade do sistema bacia hidrografica, estabilidade espacial, tipo de vegetação e cobertura e caracteristicas do solo. Estes fatores outros fatores determinam a faixa de uso dos modelos "1 umped".

Destacam-se os modelos desenvolvidos por BERGSTRON (1980), BLIDARU et alii (1980), BOYD \& BUFILL (1989), DEVILLEZ \& LAUDELOUT (1986), DISKIN et alii (1973), FERSI (1980), HAAN (1972), HOLECEK (1988), KUUSISTO (1980), LUNDQUTST (1980), MENDEL (1980), WARD (1984a), WARD (1984b), os estocásticos CALDER (1986a), HIGGINS (1981) ROGERSON (1976).

c) Modelos "black box" : Segundo wOOD \& $0^{\circ}$ CONNELL (1985) estes modelos tem como objetivo identificar as relaçóes entre a chuva ("entrada") e o deflúvio ("saida") sem tentar descrever nenhum mecanismo pelo qual esta transformação felta.

Este caminho \& frequentemente referido aos sistemas aproximados como altamente dependente de técnicas relacionadas a sistemas tebricos, como por exemplo a hidrografa unitária. 


\title{
3. O SISTEMA JOHNSON DE DISTRIBUICÃO
}

\author{
"A ciencia nos oferece copia \\ do real. Ela nos dá apenas \\ modelos hipoteticos e \\ provisórios da mesma".
}

(R. ALVES)

A inclusão deste capitulo se faz necessario na medida em que a aplicação do Sistema Johnson de Distribuição em estudos de hidrologia, como utilizado nesta dissertação e pouco conhecido, fornecendo o embasamento teorico necessário para a modelagem.

No entanto, como poderá ser visto na sequencia, é um sistema de grande flexibilidade com relação às formas das distribuiçбes que podem ser obtidas, como também é de fácil manuseio no tocante a estimativa dos parámetros.

estrutura:

A sequéncia apresentada tem a seguinte

3.1. Fundamentos do Sistema Johnson de Distribuiçăo

3.2. Os Sistemas
3.3.1. Sistema $S_{L}$
3. 3.2. Sistema $S_{B}$
3. 3. 3. Sistema $S_{U}$

3. 3. Apl i caçZes 


\subsection{Fundamentos do Sistema Johnson de Distribuição}

A distribuição Normal assume posição de destaque dentro da estatística e inúmeras variáveis encontradas na natureza assumem distribuição similar à Normal.

No entanto outras variáveis, como a distribuição da vazão no tempo, apresentam distribuição diferente da Normal. Pode-se então trabalhar com ditribuiçôes como : Gama, Exponencial, Weibull, Gausiana Inversa, Pareto, Cauchi, Logistica, Laplace, Lognormal, Beta, Uniforme, $F, t, x^{2}$, dentre outras, CJOHNSON \& KOTZ, 19703 .

Além das distribuiçoses anteriormentes citadas, existem sistemas de distribuição que têm o poder de cobrir uma grande variedade de formas. Dos sistemas de distribuição existentes, destaca-se o proposto por JOHNSON (1949). O sistema de curvas de frequiencia gerado por métodos de translação.

A idéa básica consiste em, a partir de uma variável normal reduzida (z), obter uma transformação linear do tipo:

$$
z=\gamma+\delta \cdot f\left(\frac{x-\varepsilon}{\lambda}\right)
$$

Onde os parametros $\delta$ e $\gamma$ são responsávels pela forma, $\lambda \epsilon$ uma fator de escala e um parámetro de localização. A ideia pode ser tambem representada graficamente como na Figura 8 .

o objetivo obter a distribuição de $x$ ou $y$, onde a função $f$ seja preferencialmente de forma simples e com determinado número de parâmetros. Pode-se também escrever:

$$
y=\frac{(x-\epsilon)}{\lambda}
$$




$$
P(y)=\left.\delta \cdot f^{\circ}(y) \cdot p(z)\right|_{z=\gamma+\delta \cdot f(y)}
$$

$$
P(y)=\frac{1}{\sqrt{2 \pi}} \cdot \delta \cdot f^{\prime}(y) \cdot \exp \left(-(y+\delta . f(y))^{2} / 2\right)
$$

O sistema de curvas de frequiencia depende da escolha da função, cujas propriedades por conveniencia prática são as seguintes (JOHNSON, 1949 ):

1) Ser uma função monótona de $y$.

23 Ser de fácil manuseio, preferencialamente com a existencia de tabelas para cáculos.

3 Contradominio pertencente ao intervalo $(-\infty, \infty)$.

$4)$ resultado do sistema de distribuição de y $C$ e consequentemente de $x$ incluira muitas distribuiçбes, se não todos, dos tipos encontrados nos dados coletados.

\subsection{Os Sistemas}

Trés sistemas ou familias podem ser obtidos da expressão (1), fazendo-se variar a função $f$ :

$$
\begin{array}{ll}
\text { Se } f(x)=\log (y) & \rightarrow S_{L} \\
\text { Se } f(x)=\log (y /(1-y)) & \rightarrow S_{B} \\
\text { Se } f(x)=\log \left(y+\sqrt{y^{2}+1}\right) \rightarrow S_{U}
\end{array}
$$

Antes de serem comentados aspectos relati vos a cada um dos sistemas de distribuição, e conveniente compararmos graficamente a flexibilidade do sistema proposto por JOHNSON (1949), em relação a outras 


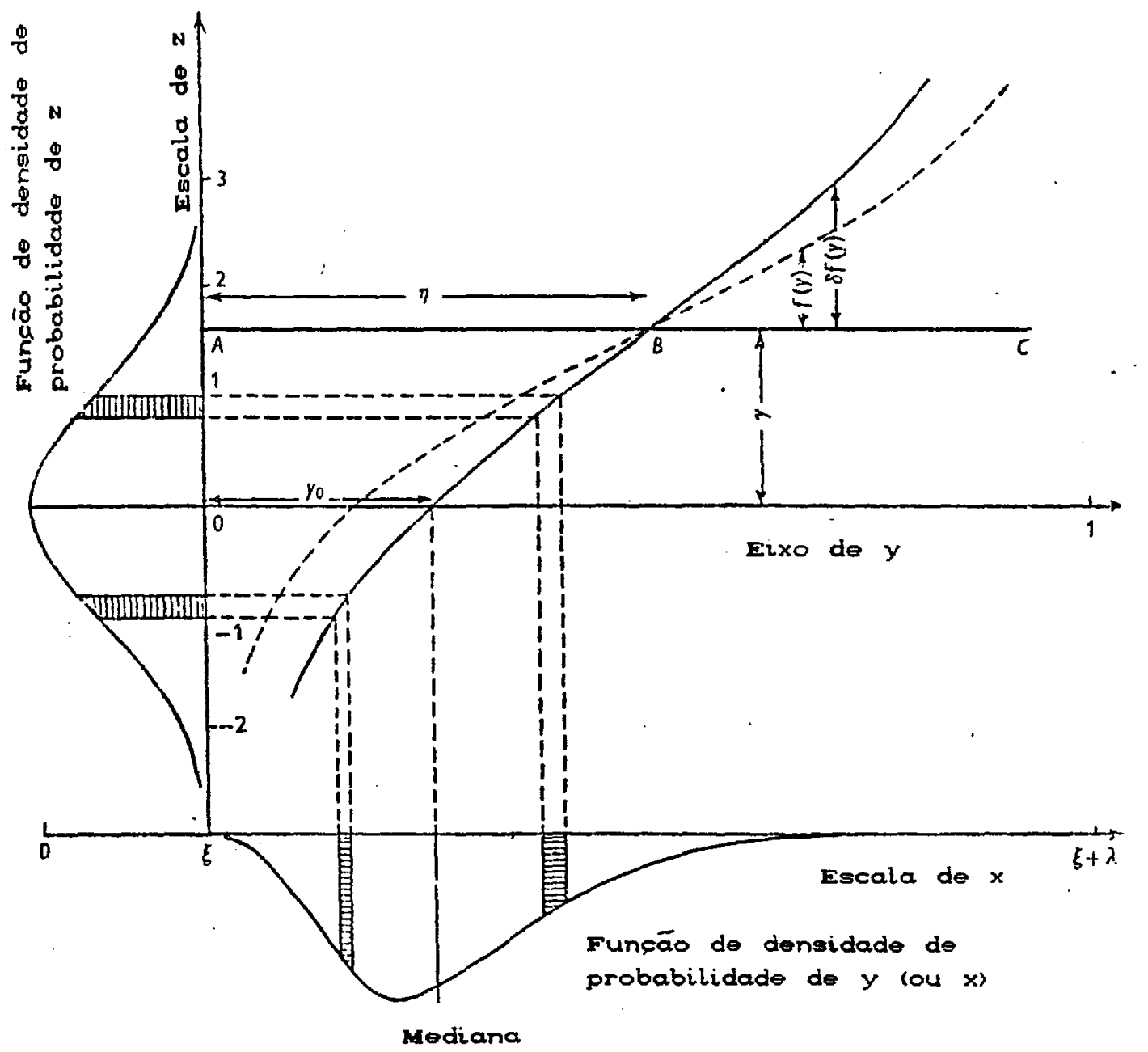
Figura 8 - Sistema de translação do Sistema Johnson.
(JOHNSON, 1949). 
distribuiçóes conhecidas. Define-se os $3^{\circ}$ e $4^{\circ}$ momentos estandartizados em relação à média crespectivamente coeficiente de assimetria e curtose $\beta_{1}$ e $\beta_{2}$ cestimadores populacionaisj e $b_{1} e b_{2} C$ estimadores amostrais), ( SHAPIRO \& GROSS, 1981):

$$
\sqrt{\beta_{1}}=\frac{E\left[(x-\mu)^{3}\right]}{\left\{E\left[(x-\mu)^{2}\right\}^{9 / 2}\right.}
$$

$$
\sqrt{\hat{\beta}_{1}}=\sqrt{b_{1}}=\frac{m_{9}}{m_{2}^{9 / 2}}
$$

onde: $m_{2} e m_{9}$ são respectivamente :

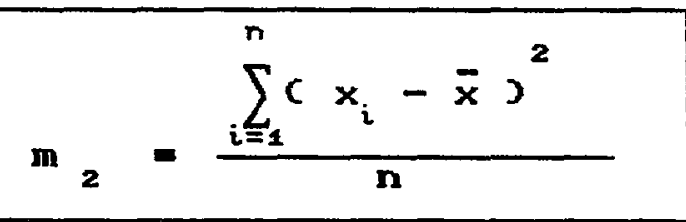

$$
m_{a}=\frac{\sum_{i=1}^{n}\left(x_{i}-\bar{x}\right)^{3}}{n}
$$


e $\beta_{2}:$

$$
\sqrt{\beta_{2}}=\frac{E\left[(x-\mu)^{4}\right]}{\left\{E\left[(x-\mu)^{2}\right\}^{2}\right.}
$$

$$
\sqrt{\hat{\beta}_{2}}=\sqrt{b_{2}}=\frac{m_{4}}{m_{2}^{2}}
$$

Pode-se plotar $\beta_{1} \odot \beta_{2}$ num sistema de eixos cartesianos (Figura 9.), sendo possivel então localizar alguma distribuição padrão previamente estudada. Por exemplo, a distribuição Normal tem $\beta_{1}=0$ e $\beta_{2}=3$, coordenadas $(0,3)$, sendo representada por um ponto. Analogamente, a distribuição exponencial e representada pelas cooderiadas $(4,9)$. Todas as distribuiçóes que não têm parametros de forma são representadas por um único ponto. Nestes casos não se tem uma relação entre $\beta_{1} e \beta_{2}$ e seus respectivos parametros, sempre resultanado em simples pontos no sistema de eixos. Distribuiçбes com um único parametro de forma estão situadas sobre uma IInha reta no plano cartesiano, CBOWMAAN \& SHENTON, 19493. Por exemplo a distribuição Gama tem a seguinte relaçăo entre $\beta_{1} \ominus \beta_{2}$ :

$$
\beta_{2}=3 \cdot\left(1+\beta_{1}{ }^{\prime} 2\right)
$$

Já as distribuiç̧̃es que têm dois parámetros de forma cobrem uma reglão no plano, uma dimensão superior Cbidimensionals, o caso da familia Beta, situada entra as 
retas :

$$
\beta_{2}=3 \cdot\left(1+\beta_{2}-2\right)
$$

$$
\beta_{2}=1+\beta_{2}
$$

No caso do sistema Johnson de Distribuicão, este cobre todo o plano, exceto a região impossivel, englobando assim uma grande variedade de formas de distribuiços, CFigura 103.

- metodo de ajuste cpercentis, momentos e máxima verossimilhançal difere para cada uma das tres familias. No entanto é necessario quando de posse dos dados, decidir qual das tres subfamilias selecionar CSHAPIRO E GROSS, 1981). SLIFKER \& SHAPIRO (1980), apresentam um procedimento que vem auxiliar a escolha do sistema e consequientemente na estimativa dos parametros :

1 Escolher um valor de $z>0$, onde $z \in a$ variável normal reduzida. A escolha feita levando-se em conta dois aspectos: o número de observações e cpara subsequiente usol os pontos nos quais os percentis serão igualados. No procedimento de estimativa a igualdade ocorre nos percentis correspondendo a $-3 z,-z, z, 3 z$. Entretanto $z$ sera escolhido de forma que o ajuste cubra as áreas de interesse para um conjunto de dados de tamanho moderado, para $n<1000$, um valor de $z$ menor que 1 seria selecionado. A escolha de um $z>1$ dificulta a estimativa dos pontos de percentagem $\pm 3 z$, isto $e$, pontos al em dos percentis 0,0014 e 0,9986. Valores de $z$ próximos a 0.5 são recomendados para amostras de tamanho moderado. Por exemplo a escolha de $z=$ 0.524 , que produz $3 z=1.572$, significa utilizar os pontos 5.8\%, 30\%, $70 \%$ e $94 \%$. 


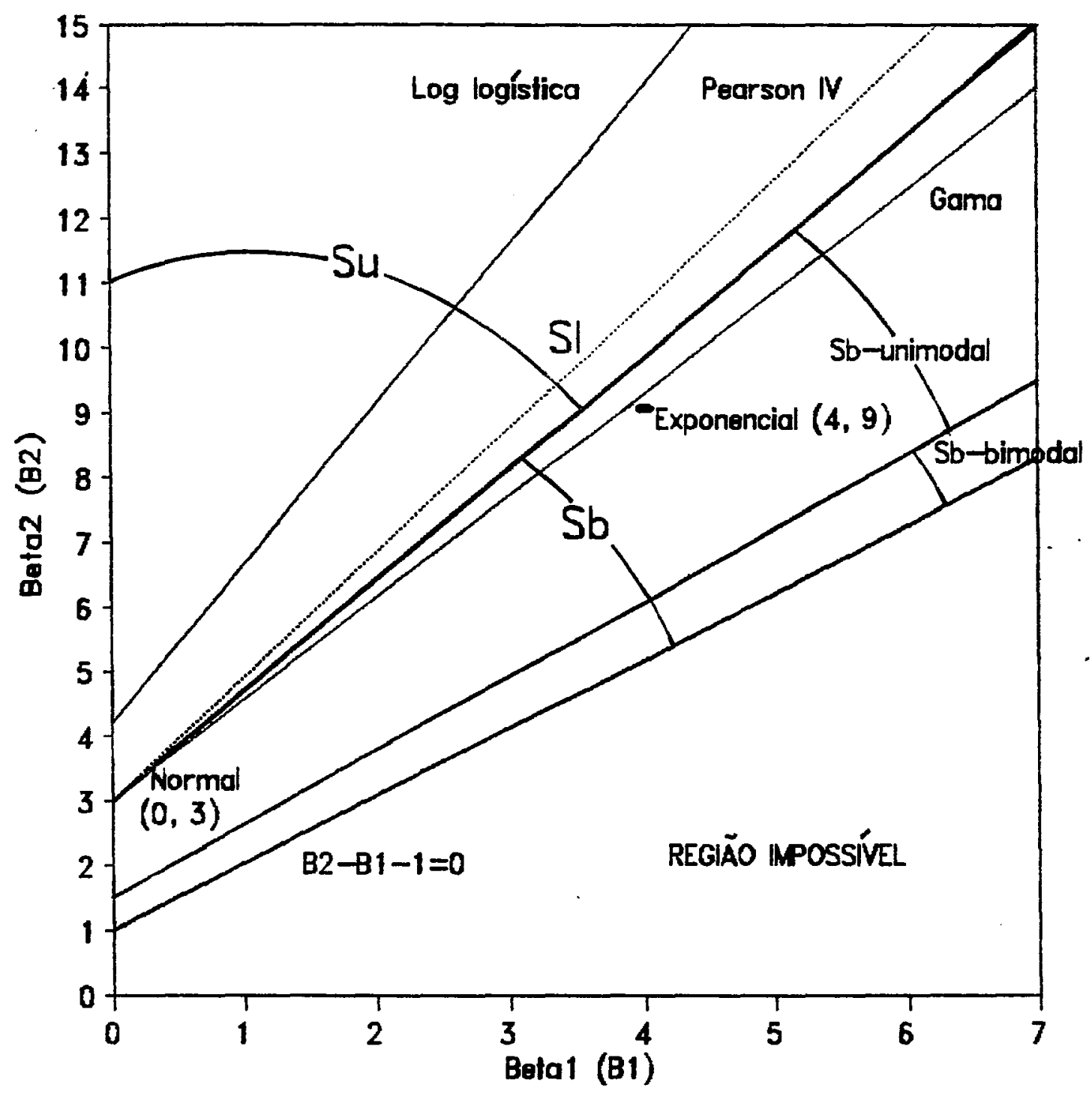

Figura 9 - Representação de algumas distribuiçoses no plano formado pelos coeficientes de assimetria curtose ( 131 e $\beta_{2}$ ), CJOHNSON, 19492. 
2) Uma vez escolhido $z$, determinar a partir da tabela da Normal os valores de $F(3 z), F(z), F(-z)$, $F(-3 z)$.

3 Obter dos dados de $x$ os percentis correspondentes à $\pm z= \pm 3 z$, resol vendo para 1 a equação :

$$
\frac{i}{n}=F(k)
$$

onde $k=+-3 z e+-z e n$ é o tamanho da amostra. Assim, $x_{k}=1$ gual a observaçăo de $i^{\text {th }}$ ordem correspondendo ao val or de $k$.

Se $i$ não for inteiro, interpol ação é utilizada.

4 Computar os seguintes valores :

$$
\begin{aligned}
& \mathbf{m}=x_{9 z}-x_{z} \\
& \mathbf{n}=x_{-z}-x_{9 z} \\
& p=x_{z}-x_{9 z}
\end{aligned}
$$

5) Para a razão discriminante $\left(m_{0} \mathbf{n} / \mathrm{p}^{2}\right.$ :

$$
\begin{aligned}
& \left(m_{0} n / p^{2}=1 \text { utilizar sistema } S_{L}\right. \\
& \left(m_{0} n / p^{2}>1 \text { utilizar sistema } S_{U}\right. \\
& \left(m_{0} n / p^{2}<1 \text { utilizar sistema } S_{B}\right.
\end{aligned}
$$



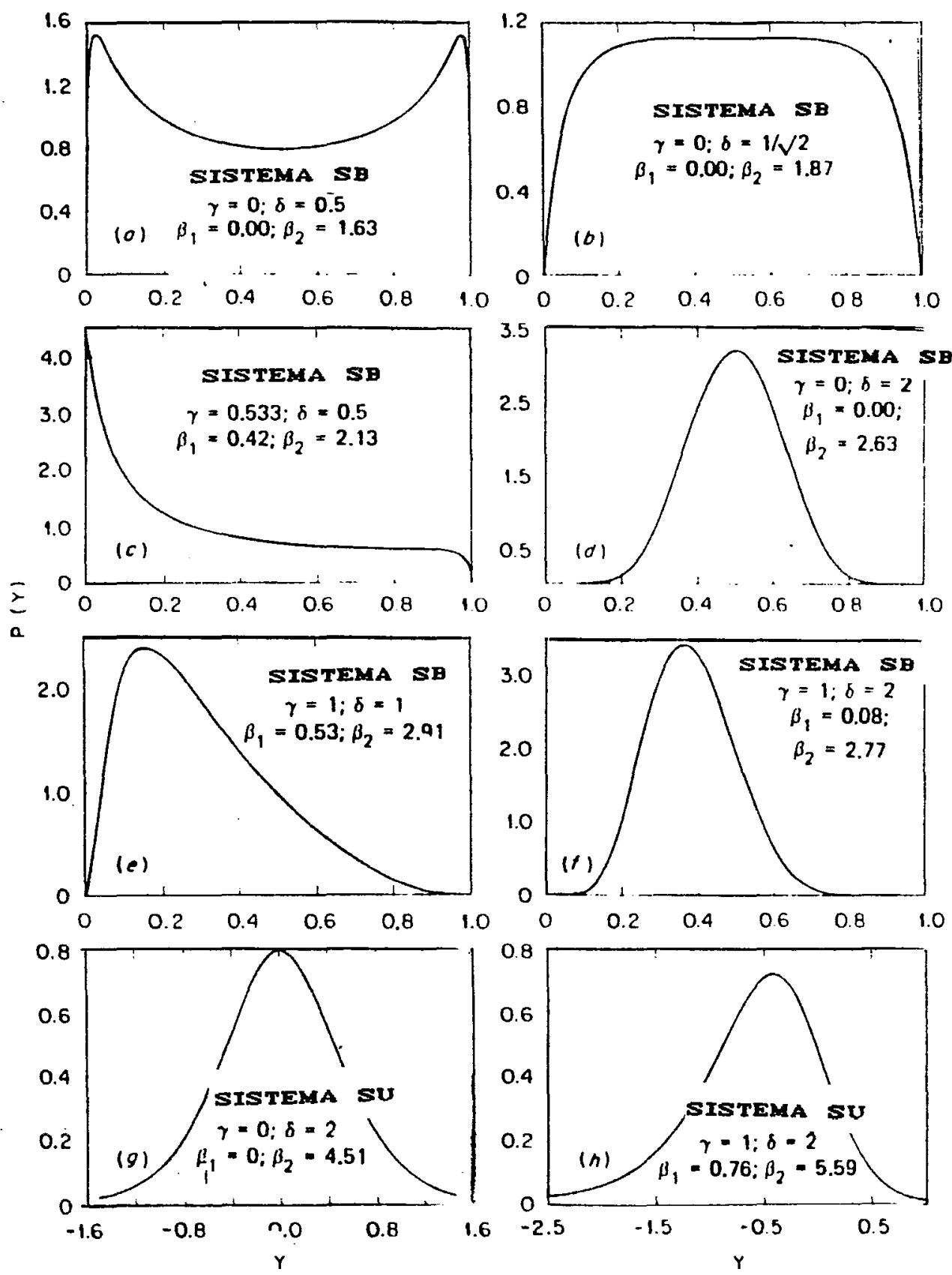

Figura 10 - Algumas possiveis distrbuiços apresentadas pelo Sistema Johnson de Distribuiç̃o CBOWMAAN \& SHENTON, 1983 . 
3.2.1. Sistema $S_{1}$

Neste sistema tem-se (JOHNSON, 1949):

$$
f(x)=\log \left(\frac{x-\varepsilon}{\lambda}\right)
$$

$z=\gamma+\delta \cdot \log (y)$

$$
P(y)=\frac{\delta}{\sqrt{2 \pi} \cdot y} \cdot \exp \left(-(y+\delta \cdot \log (y))^{2} / 2\right)
$$

$\operatorname{com} y>0$.

Os trés métodos principais de estimativa apresentados em 3.2. são discutidos por ELDERTON (1969), JOHNSON (1949), ORD (1972), SHAPIRO \& GROSS (1981).

De (23) :

$$
\begin{aligned}
z=\gamma+\log \left(\frac{x-\varepsilon}{\lambda}\right) \\
\\
z=\gamma-\delta \cdot \log (\lambda)+\delta \cdot \log (x-\varepsilon)
\end{aligned}
$$

Cujos parametros são $\delta=\sigma^{-1}, \varepsilon, \alpha=\gamma-\delta . \log (\lambda)$ a) Momentos : Fazendo $w=\exp \left(\delta^{2} / 2\right), p=\exp (-\alpha / \delta) \rightarrow$ 


$$
\rightarrow \quad \begin{aligned}
& \mu_{1}^{\prime}=w p+a \\
& \left.\mu_{2}=(w p)^{2} c w^{2}-1\right) \\
& \left.\beta_{1}=C w^{2}-13 \cdot C w^{2}+2\right)^{2} \\
& \beta_{2}=w^{\theta}+2 \cdot w^{6}+3 \cdot w^{4}-3
\end{aligned}
$$

Fazendo $t=\left(w^{2}-1\right)$ a expressão para $\beta_{1}$ produz :

$$
\begin{aligned}
& t^{3}+3 . t-\sqrt{\beta_{1}}=0 \\
& \rho=\sqrt{\left(\mu_{2} / t^{2}\left(t^{2}+1\right)\right.} \\
& \varepsilon=\mu^{\prime}-\sqrt{\mu_{2} / t}
\end{aligned}
$$

Quando $\varepsilon \in$ conhecido, somente os dois primeiros momentos săo necessarios. Estes estimadores são totalmente eficientes com $\alpha^{2}=0$, mas com $\alpha^{2}=0.4$ a eficlencia cai para 50\% CORD, 1972 .

b) Máxima verossimilhança : Os estimadores $\hat{\alpha}$ e $\hat{\alpha}$ são sol uçōes das equaçס̃es :

$$
\begin{aligned}
& \hat{n}=\Sigma\left(u{ }_{i}{ }^{2}\right. \\
& \text { n. }(\hat{\lambda}+\hat{\alpha})=\Sigma\left(u{ }_{i}\right)^{2}
\end{aligned}
$$

onde $u_{i}=\operatorname{Ln}\left(x_{i}-\theta\right) i=1,2, \ldots . ., x$. Se $\varepsilon=$ desconhecido deve-se adicionar a equação : 


$$
\left.\hat{c \sigma^{2}}+\alpha \partial \cdot \Sigma c x_{i}-\varepsilon\right)^{-1}=-\sum u_{i} c x_{i}-\hat{\varepsilon} 3^{-1}
$$

c) Percentis : (SHAPIRO \& GROSS, 1981 )

$$
\begin{aligned}
& \hat{\varepsilon}=\frac{\left(x_{p} \cdot x_{1-p}-x_{0.50}^{2}\right)}{\left(x_{p}+x_{1-p}-2 . x_{0.50}\right)} \\
& \hat{\lambda}=\frac{z+\hat{1-p}}{\operatorname{Ln}\left(\left(x_{1-p}-\hat{\varepsilon}\right)\left(x_{0-5}-\hat{\varepsilon}\right)\right)}
\end{aligned}
$$

onde $x_{p} \leqslant 0$ ponto de $p$-esima percentagem, $z_{p} \leqslant 0$ p-ésimo percentil da variável da distribuicão Normal padrão.

Segundo ORD (1972) quando \& conhecido os pares de percentis (27,73) produz 65 a $81 \%$ de eficiencia assintotica para $\lambda$, enquanto 0 par $(7,93)$ produz uma eficlencia inferior a $65 \%$.

\subsubsection{O Sistema $S_{B}$}

Os momentos são bastante complicados para serem estimados algebricamente, como fica evidenciado em JOHNSON (1949) \& DRAPER (1952).

Se ambos os pontos são conhecidos $(\varepsilon$ e $\lambda+\varepsilon)$ - os dados estão por extenso, a estimativa de máxima verossimilhança produz CJOHNSON, 1949; PHIEN \& JIVAJIRAJAH, $1984 b)$ : 


$$
\hat{\gamma}=\frac{-f}{S_{f}}
$$

$$
\hat{\delta}=\frac{1}{S_{i}}
$$

$$
\bar{f}=n^{-1} \cdot \sum_{i=1}^{n}\left(f_{i}\right)
$$$$
\left.S_{r}^{2}=n^{-1} \cdot \sum_{i=1}^{n} C f_{i}-\overline{f^{2}}\right)^{2}
$$

$e$

sendo :

$$
f_{i}=\log \left(\frac{x-\varepsilon}{\varepsilon+\lambda+x_{i}}\right)
$$

No entanto para os dados distribuidos em classe, existe a necessidade de correção, prcedimento de dificil manipulação CJOHNSON,19493. Neste caso o mais indicado é o método dos percentis, indicado por SHAPIRO \& GROSS (1981) :

$$
\hat{\delta}=\frac{z}{\left.\cosh ^{-1} c \sqrt{(1+p+n)(1+p / m) / 2}\right)}
$$




$$
\hat{\gamma}=\hat{\delta} \cdot \sinh ^{-1}\left[\frac{(p / n-p / m) \cdot \sqrt{(1+p / m)(1+p / n)-4}}{2 \cdot\left(p^{2} /(m \cdot n)-1\right)}\right]
$$

$$
\hat{\imath}=\frac{x_{z}+x_{-z}}{2}-\frac{\hat{\lambda}}{2}+\frac{p \cdot(p / m-p / n)}{2 \cdot\left(p^{2} /(m \cdot n-1)\right.}
$$

\subsubsection{O Sistema $S_{u}$}

Sejam as variaveis definidas como :

$$
w=\exp \left(-\delta^{2}-2\right)
$$

$$
\rho=-(\gamma / \delta)
$$

Assim os momentos são :

$$
\mu_{i}^{\prime}=-w \cdot \operatorname{Sinh}(\rho)
$$

$\mu_{2}=\left(w^{2}-1\right) \cdot\left(w^{2} \cdot \operatorname{Cosh}(2 p)+1\right) / 2$

$$
\mu_{g}=-w \cdot\left(w^{2}-1\right) \cdot C w_{0}^{2}\left(w^{2}+2\right) \cdot \operatorname{Sinh}(3 \rho)+\operatorname{Sinh}(\rho) 3 / 4
$$




$$
\mu_{4}=\left(w^{2}-1\right)^{2} \cdot\left(w^{4} \cdot\left(w^{8}+2 \cdot w^{6}+3 \cdot w^{4}-3\right) \cdot \operatorname{Cosh}(4 \rho)\right) / 8
$$

Para facilitar a solução para $\rho$ e $\delta$ em termos de $\beta_{1} e \beta_{2}$, um ábaco desenvolvido por JOHNSON (1949), e posteriormente estudos desenvolvidos por LESLIE (1959) IOHNSON (1965), fornecem $\gamma=\delta$ para $\beta_{1} e \beta_{2} \cdot 0$ método da máxima verossimilhança de dificil manipulação, CORD 1972; PHIEN \& HIRA 1983), sendo o metodo dos percentis mais facilmente utilizável CSHAPIRO \& GROSS, 1981):

$$
\hat{\delta}=\frac{2.2}{\cosh ^{-1}[((m p)+(n / p)) / 2]}
$$

$$
\hat{\gamma}=\hat{\delta} \cdot \sinh ^{-1}\left[\frac{n / p-m p}{2 \cdot \sqrt{\left(m_{0} n\left(p^{2}\right)-1\right)}}\right]
$$

$$
\hat{\lambda}=\frac{2 p \sqrt{\left(m-n / p^{2}\right)-1}}{[m p+n p-2] \cdot \sqrt{(m p+n p+2)}}
$$

$$
\hat{\varepsilon}=\frac{x_{z}+x_{-z}}{2}+\frac{p(m / p-m / p)}{2 \cdot(m p+n / p-2)}
$$

Se $m<n, \gamma$ e positivo; de outro modo negativo. 
3. 3. Aplicaçỡes

HILL et alii (1976) apresentam um algoritmo para ajuste dos tres subsistemas $\left(S_{B}, S_{L}, S_{\mathbf{U}}\right.$ ) em linguagem Fortran. Para o subsistema $S_{B}$ os autores utilizaram a aproximação de Goodwin's para integrais de faixa infinita dupla, utilizando integrandos: $g(x)=f(x) \cdot \exp \left(-x^{2}\right)$, suger 1do por DRAPER (1952).

Na Tabela 2 estão resumidas algumas aplicaçס̃es dos sistemas de translação de Johnson e Pearson.

Tabela e - Aplicações dos sistemas de translação.

\begin{tabular}{|c|c|c|}
\hline Autor & Variárel & Distribuiçăo \\
\hline JOHNSON (1949) & graus de nebulosidade & Pearson $I$ \\
\hline JOHNSON (1849) & $\begin{array}{l}\text { idade de mães até } 0 \\
\text { parto }\end{array}$ & Pearson. I \\
\hline JOHNSON (1949) & comprimento de grăos & $S_{\text {U }}$, Pearson IV \\
\hline MAGE (1980) & concentração de $\mathrm{CO}_{2}$ & $S_{B}$ \\
\hline $\begin{array}{l}\text { SLIFKER \& SHAPIRO } \\
(1980)\end{array}$ & resistencia elétrica & $S_{B}$ \\
\hline $\begin{array}{l}\text { MCMAHON \& } \\
\text { SRI KANTHAN (1981) }\end{array}$ & $\begin{array}{l}\text { análise de freqúencia } \\
\text { de cheias }\end{array}$ & Log pearson III \\
\hline $\begin{array}{l}\text { SHAPIRO \& GROSS } \\
\text { (19813 }\end{array}$ & corrente eletrica & $S_{B}$ \\
\hline $\begin{array}{l}\text { SRIKANTHAN \& } \\
\text { MCMAHON }(1981)\end{array}$ & pico anual de cheias & Log pearson III \\
\hline $\begin{array}{l}\text { PHIEN \& CHOW } \\
\text { (1983) }\end{array}$ & dados de vazão anual & $\mathbf{S}_{\mathbf{B}}$ \\
\hline $\begin{array}{l}\text { PHIEN \& } \\
\text { JIVAJIRAJAH (1984a) } \\
\text { PHIEN \& }\end{array}$ & dados de vazăo anual & $S_{B}$ \\
\hline
\end{tabular}


4. METODOLOGIA

$$
\begin{aligned}
& \text { "Metodos contém sempre una } \\
& \text { metafisica; Inconscientemente, } \\
& \text { eles revelam conclusbes que } \\
& \text { frequientemente afirmam ainda não } \\
& \text { conhecer". }
\end{aligned}
$$

(A. Camus)

4.1. Área experimental - O Sistema

$$
\begin{aligned}
& \text { 4.1.1. - Localização } \\
& \text { 4.1.2. - Relevo e caracteristicas físicas } \\
& \text { 4.1.3. - Solos } \\
& \text { 4.1.4. - Vegetação } \\
& \text { 4.1.5. - Clima }
\end{aligned}
$$

4. 2. - Fundamentos do mstodo utilizado

4.2.1. - Hipótese básica

4. 2. 2. - Identificação e caracterização das churas - A Entrada

4.2.3. - Identificação e caracterização das Hidrografas - A Salda

4. 2. 4. - A Model agem - MSHJ e MSHA 
4. 1. Area experimental - O Sistema

4.1.1. - Localização

A área em estudo Cbacia experimental "D") está localizada no Parque Estadual da Serra do Mar - Núcleo de Cunha, situado entre os paralelos $23^{\circ} 13^{\prime} 28^{\prime \prime}$ e $23^{\circ} 16^{\prime} 10^{\prime \prime}$

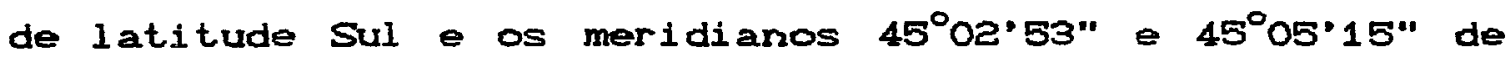
latitude $W$ Gr., apresentando uma area aproximada de 2.854,00 ha no planal to do Paraitinga, correspondendo a uma parte do alto médio vale do Paraibuna CFURIAN \& PFEIFER, 1986), (Figura 11).

4.1.2. - Relevo e caracteristicas fisicas (Figura 12 ).

A região e montanhosa e está localizado no Planalto Atlantico (FURIAN \& PFEIFER, 1986). A bacia hidrográfica experimental "D" ocupa uma área de 56,04 ha, CCICCO et alii, 1985). Na Tabela 3 estão resumidas várias caracteristicas físicas da bacia experimental " $D$ " CCICCO et alii, 1985; CICCO et alii, 19872. 


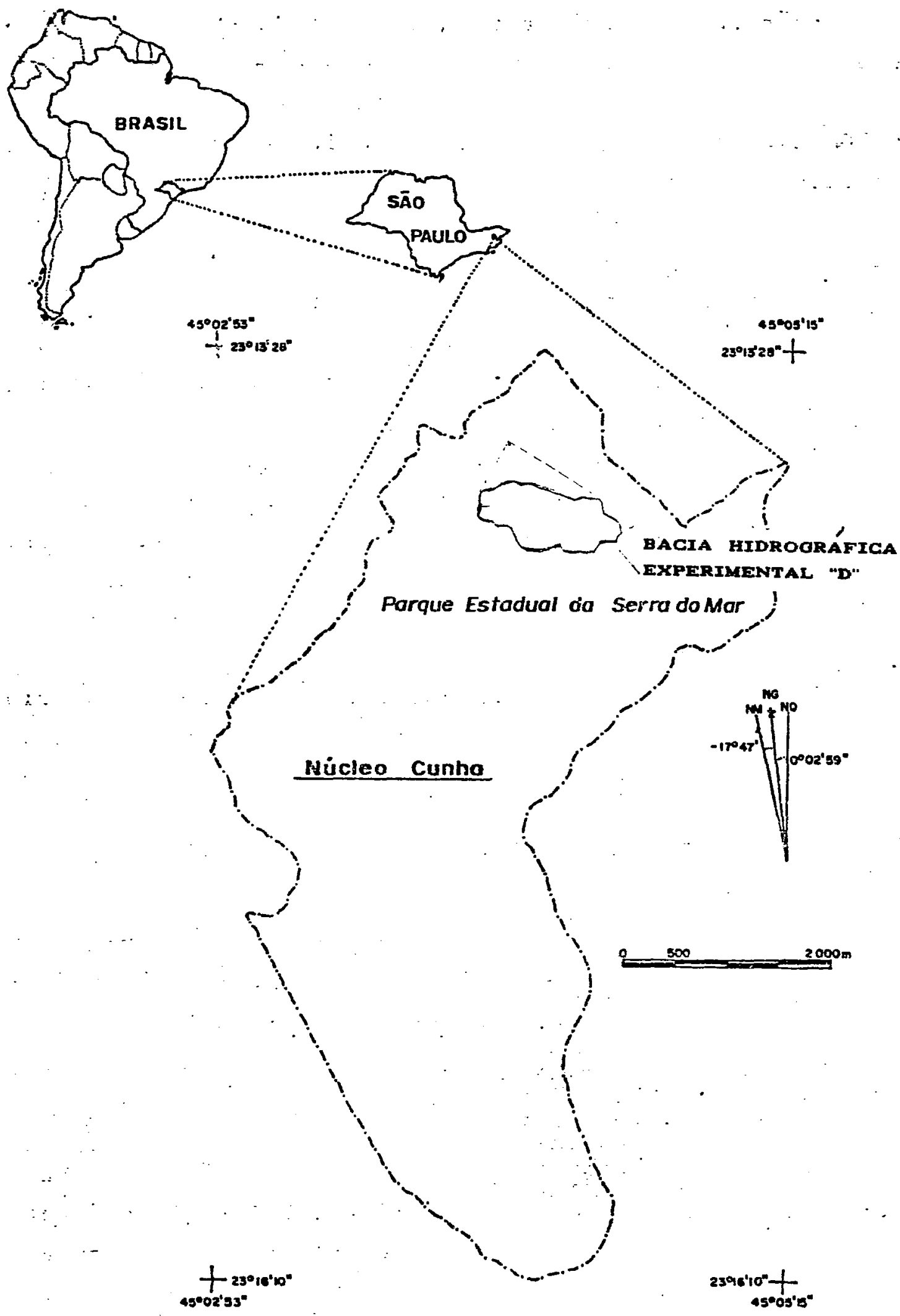

Figura 11 - Mapa de localização da área experimental. 
Tabela 3. Caracteristicas fisicas da Bacia Experimental "D" CCICCO et alii, 1985 ).

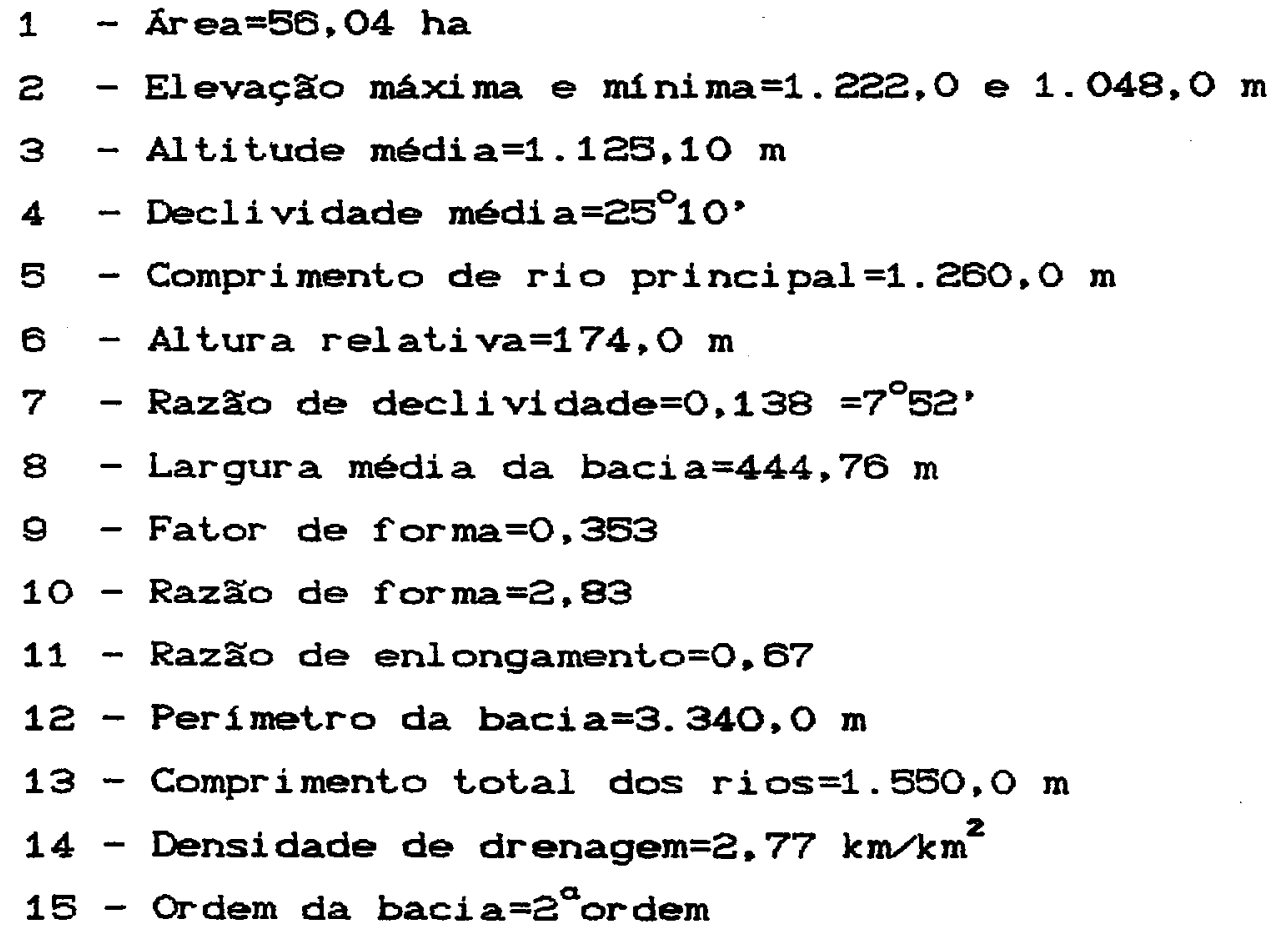




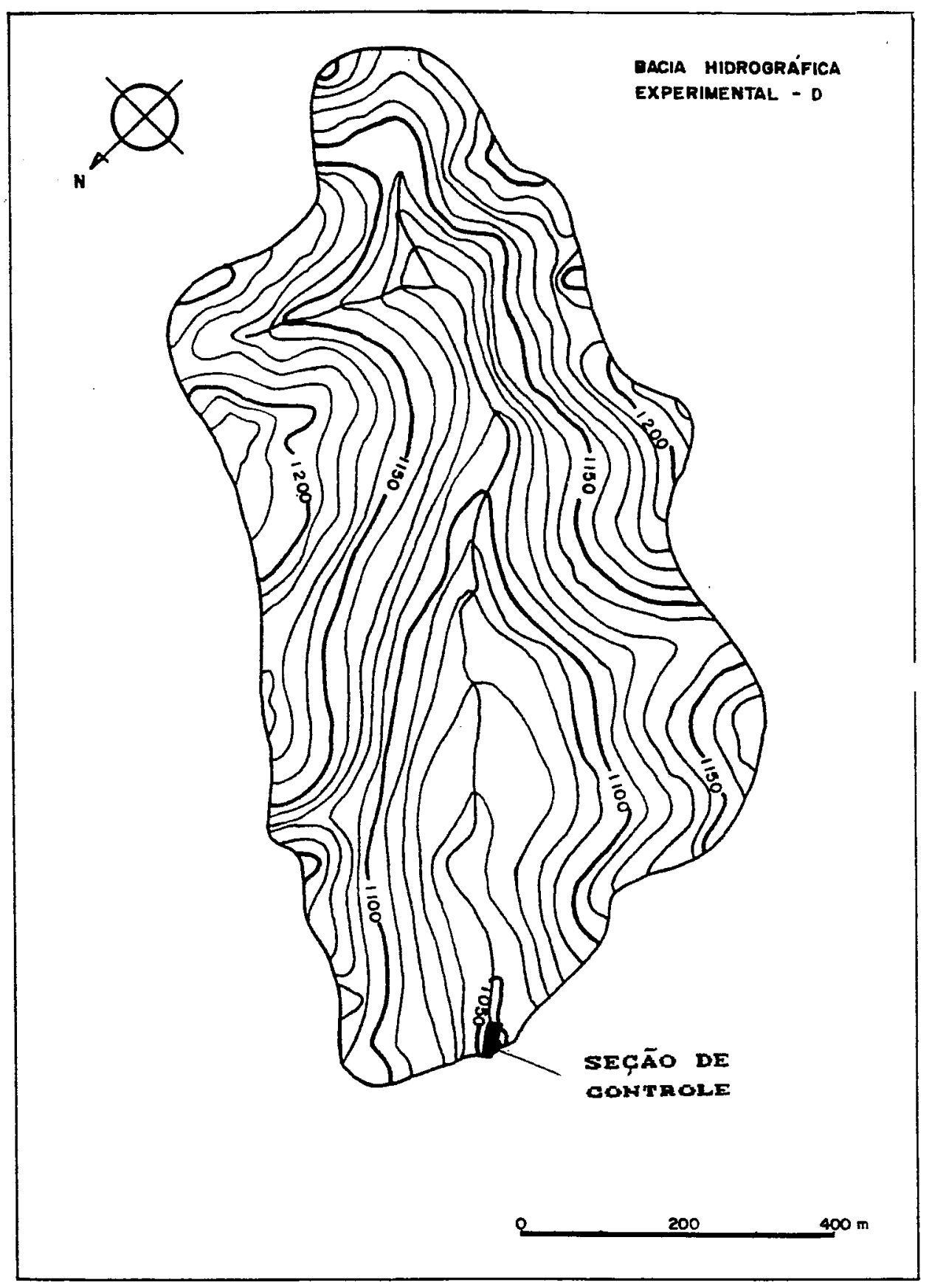

Figura 12 - Mapa planoaltimetrico da Bacia experimental "D" do Núcleo de Cunha S.P. CCICCO et alii. 19872. 
4.1.3. Solo

A unidade taxomómica e caracterizada como latossolo vermelho amarelo fase rasa (Lvr). proveniente de rochas duras e de dificll decomposição, com os gnaisses, magmatitos $\theta$ granitos. Em conseqüencia este tipo de solo quimicamente pobre, acido, relativamente raso, com textura grosseira e estrutura fraca, isto $e$, os gránulos formam uma massa fraca homogénea com muita fraca coerencia que, aliados a elevada porosidade, condiciona-lhe boa permeabili iade CFURI AN \& PFEIFER, 19865.

\subsubsection{Vegetação}

A vegetação primitiva da Floresta Latifoliada perenifólia - Mata Atlantica apresenta uma grande diversidade a nivel de familia, LEITAO FILHO (1982). A área atual encontra-se sob mata secundária, com aproximadamente 25 anos de regeneração CCICCO et alii. $1986 / 885$.

\section{1.5. Clima}

Segundo a classificação de Köppen, a regiăo apresenta clima Cwa, clima mesotermico de inverno seco com temperaturas inferiores a $18^{\circ}$ no inverno e superiores a $2 e^{\circ}$ no verão, (FURIAN \& PFEIFER, 1986).

A temperatura e umidade relativa média são respectivamente $16,5^{\circ}$ e $79,3 \%$, conforme dados observados no posto meteorológico do núcleo. A precipitaçăo média anual é de aproxi madamente $2.000 \mathrm{~mm}$, com o ciclo de periodo chuvoso compreendido entre outubro e abril e periodo seco abrange os meses de maio a setembro, CCICCO et alii, 1986/885. A Figura 13 apresenta os dados de precipitação e escoamento (média mensall no período de maio de 1982 a julho de 1983, e na Tabela 4 os dados de altura de lamina d'água, CCICCO et alii, 1987 . 


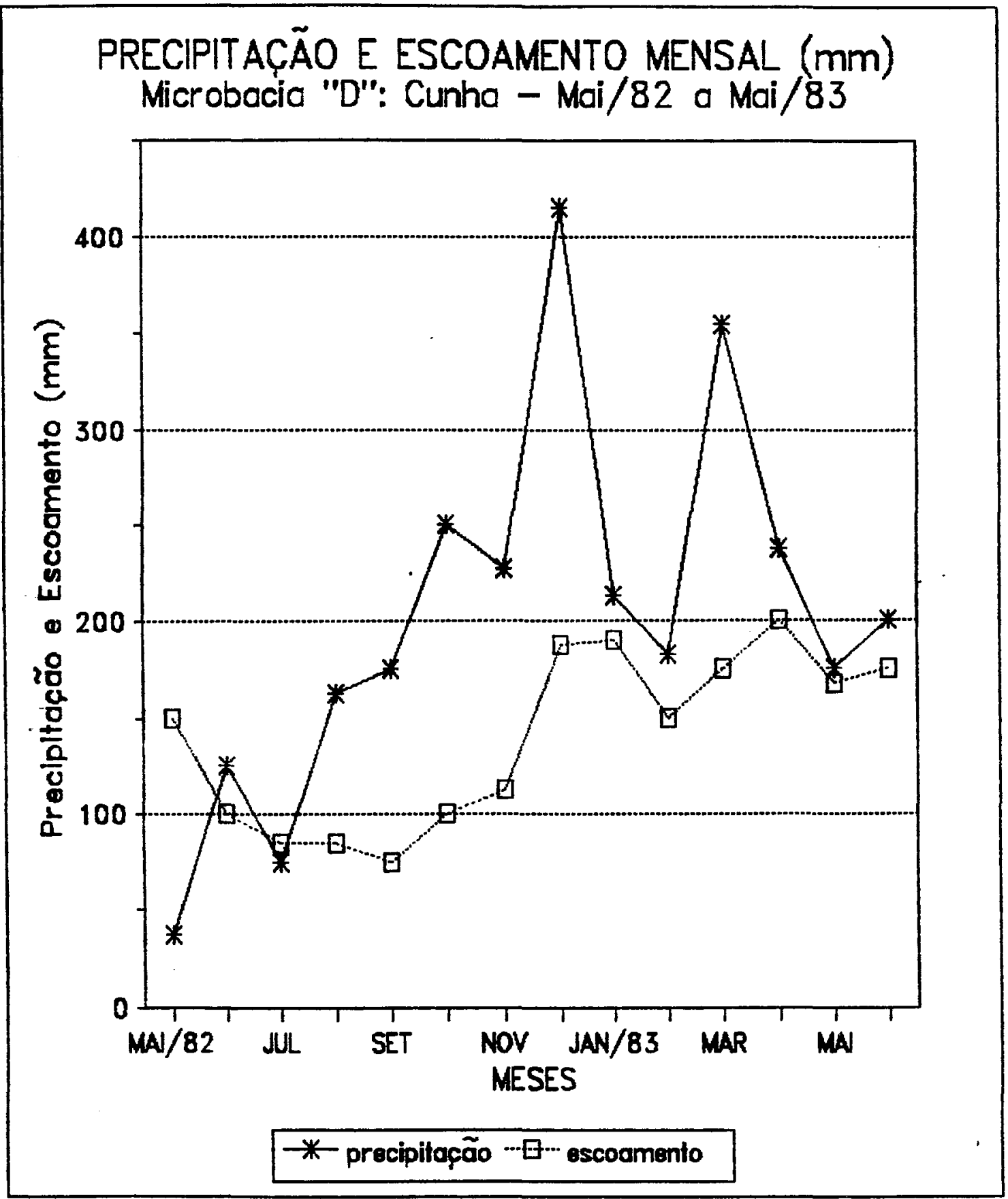

Figura 13 - Relação entre a precipitaçăo e o escoamanto mensal. CCICCO et alii, 1987 ). 
Tabela 4 - Precipitação, altura de lamina d'água e vazão mensal maxima e minima da Bacia Hidrográica "D", CCICCO et alii, 1987 ).

\begin{tabular}{|c|c|c|c|c|c|}
\hline \multirow{2}{*}{ Més } & \multirow{2}{*}{$\begin{array}{c}\text { Preci- } \\
\text { pitação } \\
\text { c mm }\end{array}$} & \multicolumn{2}{|l|}{ minima * } & \multicolumn{2}{|l|}{ máxi ma } \\
\hline & & $\begin{array}{l}\text { Altura da } \\
\text { lámina } \\
\text { d'água }\end{array}$ & $\begin{array}{l}\text { vazão } \\
(1 / s)\end{array}$ & $\begin{array}{l}\text { Altura da } \\
\text { lámina } \\
\text { d'água }(\mathrm{cm})\end{array}$ & $\begin{array}{l}\text { vazão } \\
(1 / s)\end{array}$ \\
\hline Mai / $/ 82$ & 33,5 & 3,5 & 24,47 & 5,0 & 44,84 \\
\hline$J \mathbf{u n}$ & 123,5 & 3,0 & 18,83 & 11,5 & 184,50 \\
\hline Jul & 65,0 & 2,5 & 13,82 & 6,0 & 61,11 \\
\hline Ago & 122,5 & 2,0 & 9,46 & 12,5 & 212,56 \\
\hline Set & $12 B, 0$ & 2,0 & 9,46 & 13,0 & 227,21 \\
\hline Out & 228,5 & 2,0 & 9,46 & 13,4 & 239,21 \\
\hline Nor & 209,5 & 2,7 & 15,75 & 26,5 & 761,57 \\
\hline Dez & 350,5 & 3,7 & 26,89 & 25,0 & 689,81 \\
\hline $\operatorname{Jan} / 83$ & 194,5 & 4,0 & 20,70 & 8,5 & 110,42 \\
\hline Fer & 167,5 & 3,7 & 26,89 & 14,2 & 263,96 \\
\hline Mar & 331,0 & 3,6 & 25,67 & 39,0 & $1.467,96$ \\
\hline Abr & 212,5 & 4,1 & 32,01 & 24,5 & 666,55 \\
\hline máxima & 350,5 & 4,1 & 32,01 & 39,0 & $1.467,96$ \\
\hline mí ni ma & 33,5 & 2,0 & 9,46 & 5,0 & 61,11 \\
\hline
\end{tabular}

a vazão minima e definida como a minima instantanea para cada registro e não com vazão minima diária. 


\section{2. Fundamentos do método utilizado}

\section{A.2.1. Hipótese Básica}

- método utilizado tem como pressuposição básica que dentre os fatores do meio que afetam a hidrografa, a precipitação tem papel fundamental. Deste modo as chuvas antecedentes, juntamente com suas caracteristicas cintensidade, duração e distribuição no tempos, são variáveis que podem afetar a distribuição da vazão no tempo - hidrógrafa, através de modificação por exemplo do infcio do escoamanto, do tempo de pico da hidrógrafa, e do volume de agua produzido pela bacia hidrográfica experimental.

4.2.2. Identificação e caracterizaçăo das chuvas - A entrada Para o estudo de cada hidrografa foram isoladas ate 6 (seis) eventos chuvosos compreendidos dentro de um perlodo máximo de 6 (sels) dias anteriores ao inicio do escoamento, identificados na Tabela 5 .

Para cada hidrografa identificada, a chuva zero (CHOS, a mais próxima do infcio do escoamento, foi considerada como a responsavel pela produção da hidrógrafa, acompanhadadas das chuvas anteriorés CCH1, CH2, CH3, CH4 e CHS).

A separação entre cada evento chuvoso foi feita considerando-se um periodo de tempo de pelo menos 6 horas. A distribuição de cada evento chuvoso no tempo foi feita em intervalos de tempo de 20 minutos, e para cada chuva determinou-se 6 (seis) variáveis, resumidas na TABELA 6 . 
TABELA 5 - Identificação dos eventos chuvosos

\begin{tabular}{ccc}
\hline Evento churoso & Identificação & Denominação \\
\hline 0 & CHO & Atual \\
1 & $\mathrm{CH} 1$ & $1^{a}$ anterior \\
2 & $\mathrm{CH} 2$ & $2^{a}$ anterior \\
3 & $\mathrm{CH3}$ & $3^{a}$ anterior \\
4 & $\mathrm{CH} 4$ & $4^{a}$ anterior \\
5 & $\mathrm{CH}$ & $5^{a}$ anterior \\
\hline
\end{tabular}

TABELA 6 - Variáveis consideradas em cada evento chuvoso

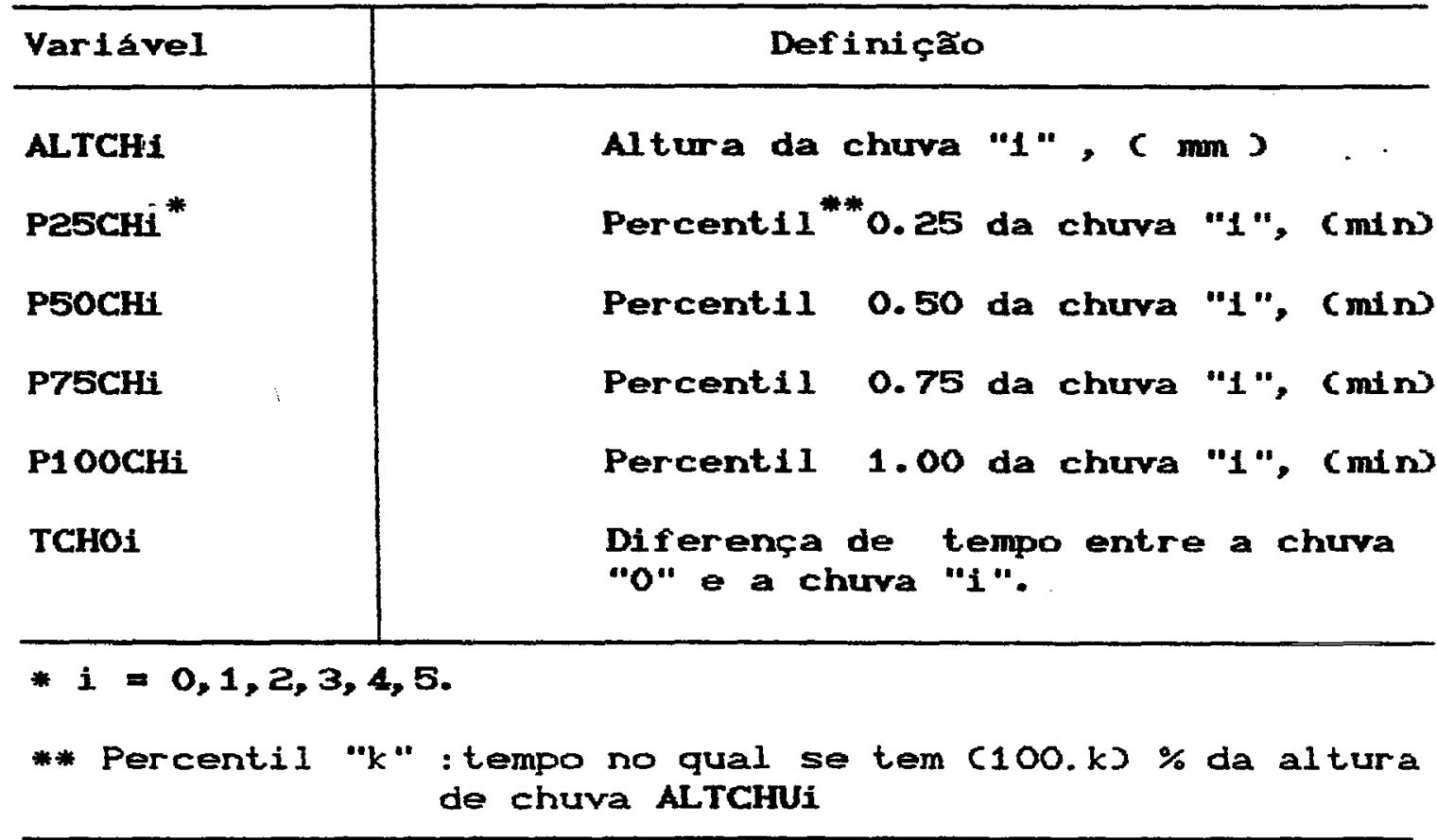

ALTCHUi: Variável obtida somando-se o número de pulsos registrados na carta do pluviógrafo multiplicado pela constante $(0,5)$, para a conversão em $\mathrm{mm}$.

PkCHi : Variável obtida após elaboração da distribuição acumulada das alturas de chuva, também denominada 
separatriz de ordem "k" $\left(S_{k}\right)$.

$$
S_{k}=L i+C . \frac{\left[n k-F_{\text {ant }}\right.}{f_{\text {sk }}}
$$

Onde :

Li = Limite inferior da classe "q" que contém a separatriz.

C = Amplitude do intervalo da classe que contém a separatriz.

k = ordem da separatriz cno caso $k=$ 0,$25 ; 0,50 ; 0,75$ e 1.003 .

Fant = Frequiencia acumulada "abaixo" da classe anterior à separatriz.

$f_{\text {sk }} \quad=$ Frequiéncia simples observada da classe que contém a separatriz.

n = Número total de observaçoes.

TCHOi : Variável determinada computando-se a diferença de tempo das chuvas anteriores em relação à chuva atual CHO Cchuva zerod, através dos respectivos centro de massa, determinado através da expressão (44).

$$
T_{c m}=\frac{\left.\sum_{i=1} \subset T_{i}\right)}{\sum_{j=1}^{n} A_{\text {ALT }}{ }_{j}}
$$

Onde :

Tam $=$ Tempo do centro de massa (minutos).

$T_{j}=$ Centro de classe do intervalo $j$.

$A L T_{j}=$ altura de chuva j relativo à classe de tempo j.

A Figura 14 resume as variáveis definidas até o momento. 

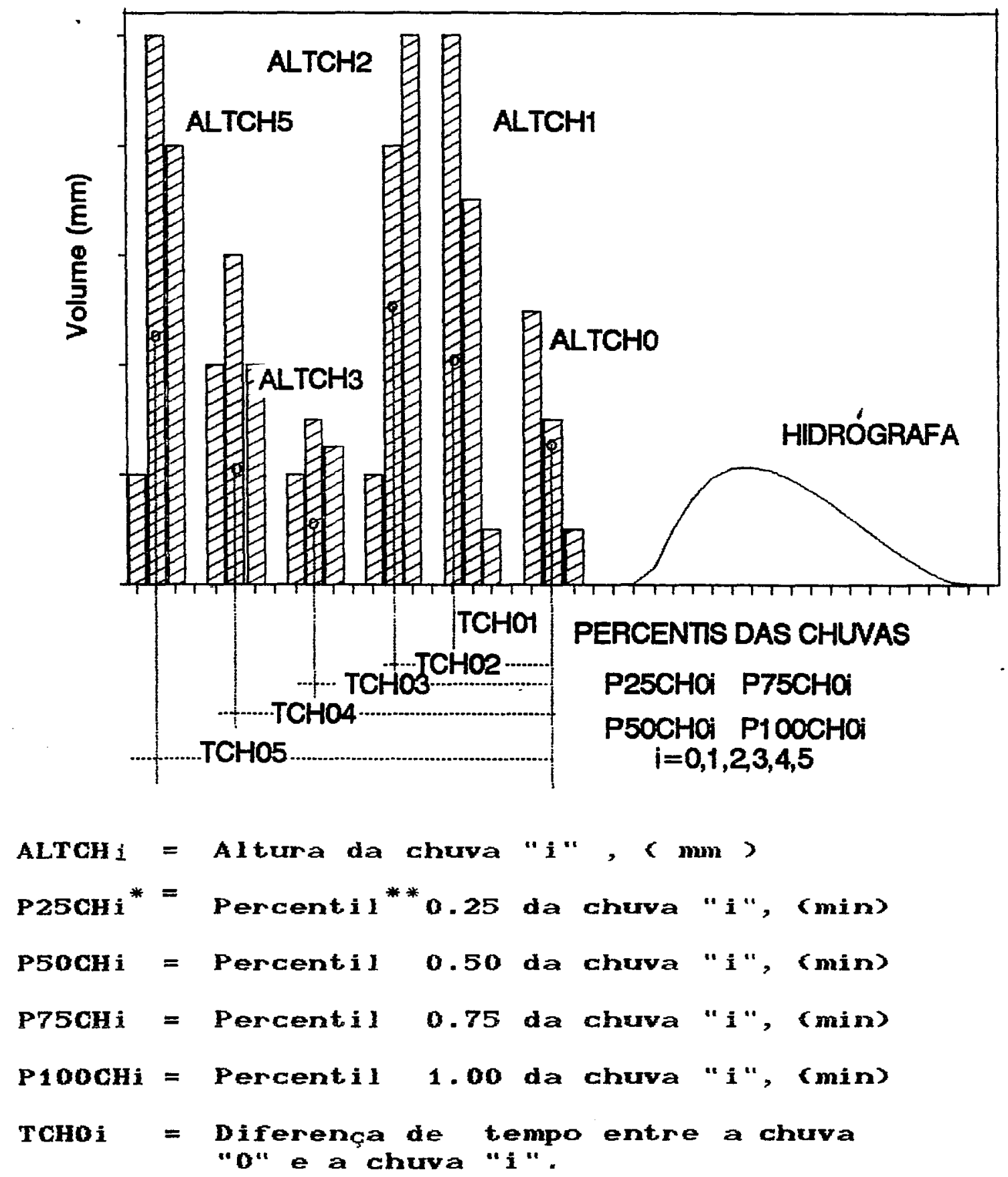

$* i=0,1,2,3,4,5$.

Figura 14 - Variáveis utilizadas no modelo de simulação. 
4.2.3. Identificação e caracterização das variáveis das Hidrografas - A saida

Ao todo 82 hidrografas foram obitidas junto ao Instituto florestal de São Paulo, abrangendo o periodo de $24 / 4 / 82$ a $20 / 12 / 83$, formando assim a massa de dados que reflete o comportamento e sensibilidade da microbacia de Cunha aos estimulos produzidos pelas condiçōes anteriores de chuva (4.2.2.).

Os dados de vazão CALTVAZ ou H caracterizados pela altura de lámina d'água (cm), distribuidos no tempo em intervalos de $20 \mathrm{~min}$., foram tratados de duas formas diferentes :

13 Numa primeira etapa, cada hidrografa foi ajustada pelo Sistema Johnson de Distribuição, utilizando-se o método dos percentis , segundo a metodologia apresentada por SLIFKER \& SHAPIRO (1980). Deste modo as hidrógrafas ficam sendo então representadas pelos parámetros de forma estimados : $\hat{\delta} e \hat{\gamma}$, e os parámetros de localização e escala $\hat{\lambda} \hat{\varepsilon}$, foram determinados para cada hidrógrafa. Posteriormente estes parámetros serão correlacionadas com as caracteristicas das chuvas anteriores de cada um dos eventos (hidrografas), Figura 15.

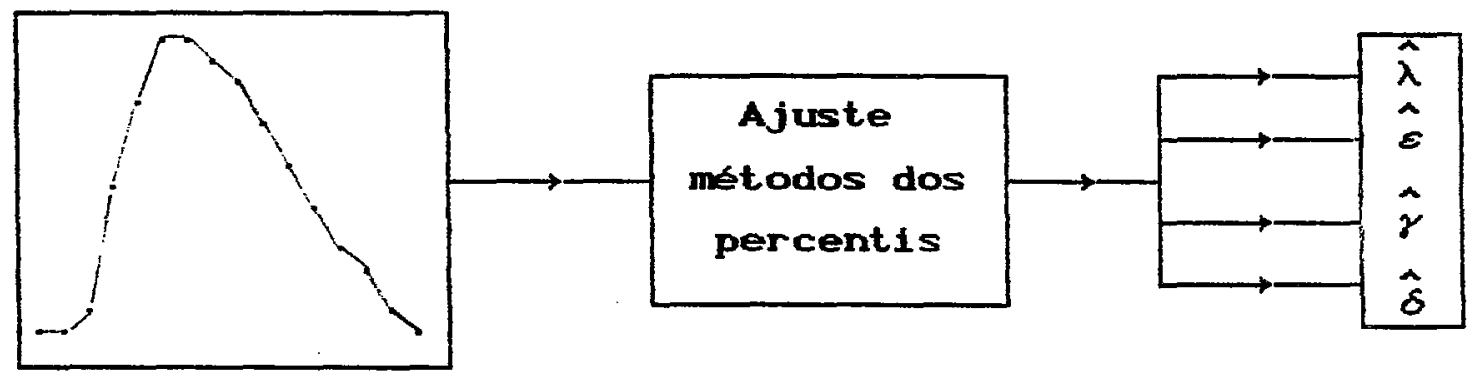

Hidrografa

Figura 15 - Representaçăo da caracterização das hidrografas após ajuste pelo metodo dos percentis. 
2) Nesta etapa cada hidrógrafa foi caracterizada pelos próprios dados de altura de lamina d'água no tempo (ALTVAZ ou Hi). Desta forma cada hidrógrafa foi modelada ponto a ponto, correlacionando-se alturas de lamina d'agua em intervalos constantes de tempo C20,40,...) com os parametros das chuvas anteriores, diferentemente da etapa anterior, onde a hidrografa foi representada por quatro parametros estimados do Sistema Johnson de Distribuição, Figura 16.

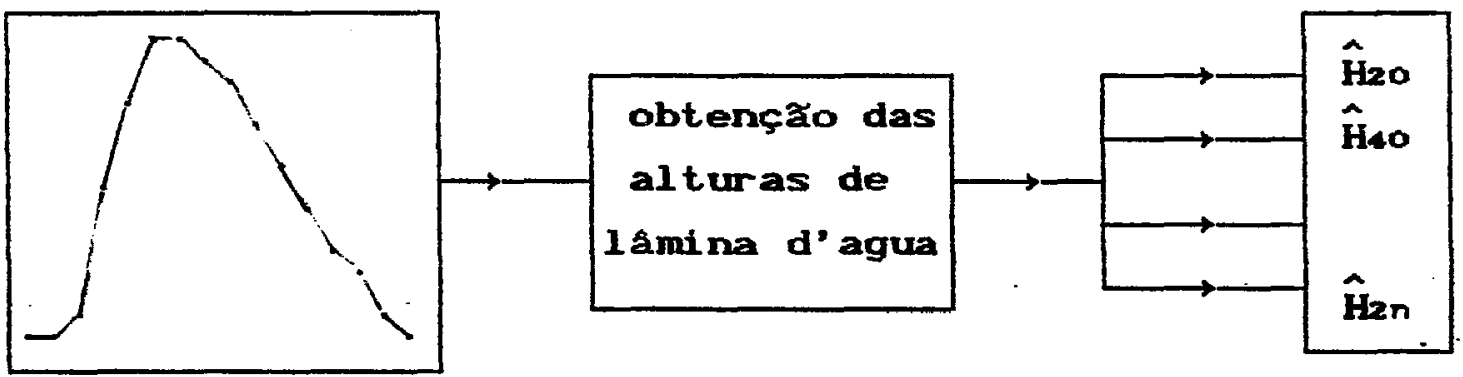

Hidrografa

Figura 16 - Representação da caracterização das hidrógrafas apos ajuste pelo método dos percentis.

\section{2. 4. A Modelagem da Hidrógrafa - MSHJ e MSHA}

A Modelagem das hidrografas foi feita utilizando-se dois caminhos, em concordancia com as etapas do item anterior 4.2.3. :

$1)$ Modelos de regressão que correlacionam os parametros (de forma) estimados das hidrógrafas, obtidos atraves do Sistema Johnson de Distribuição - método dos percentis, com as caracteristicas das chuvas (4.2.2.J. Estes modelos de regressão foram denominados de "MSHJ", para caracterizar a utilização do Sistema Johnson de 
Distribuiç̃o. Assim poderemos estimar os dois parâmetros do Sisteme Johnson de Distribuiçă, ajustando dados de vazão a partir de variáveis de entrada, facilmente mensuráveis ou simuladas, caracterizando assim a modelagem do sistema.

2) Modelos de regressão cujas variáveis dependentes são as alturas de chuva correspondente a cada intervalo de tempo, e as variáveis independentes, as caracteristicas das chuvas (4.2.2.2. Estes modelos foram denominados de "MSHA", caracterizando simulação pontual dos dados de altura de vazão.

Foram tambem determinados modelos de regressão cujas variáveis dependentes são o inicio e fim do escoamento, e as variáveis independentes as caracteristicas das chuvas.

A seguir estão representados os modelos de regressão utilizados em ambos os metodos, diferindo apenas nas variáveis dependentes utilizadas. 


$$
\phi_{k}=\text { MVAR } \cdot \theta+\mathbf{u}_{k}
$$

Onde :

$$
\begin{aligned}
& k=H_{1}, H_{2}, H_{3}, \ldots, H_{n} \text { (MSHJ) } \\
& k=\lambda, \varepsilon, \gamma, \delta \text { (MSHA) }
\end{aligned}
$$

$$
\phi=\left[\begin{array}{c}
k 1 \\
k 2 \\
\vdots \\
k n
\end{array}\right]_{n} \quad \theta_{k}=\left[\begin{array}{c}
\alpha \\
\beta_{1} \\
\beta_{2} \\
\beta_{n}
\end{array}\right]_{n+1} u_{k}=\left[\begin{array}{c}
u_{0} \\
u_{1} \\
\vdots \\
u n
\end{array}\right]_{n}
$$

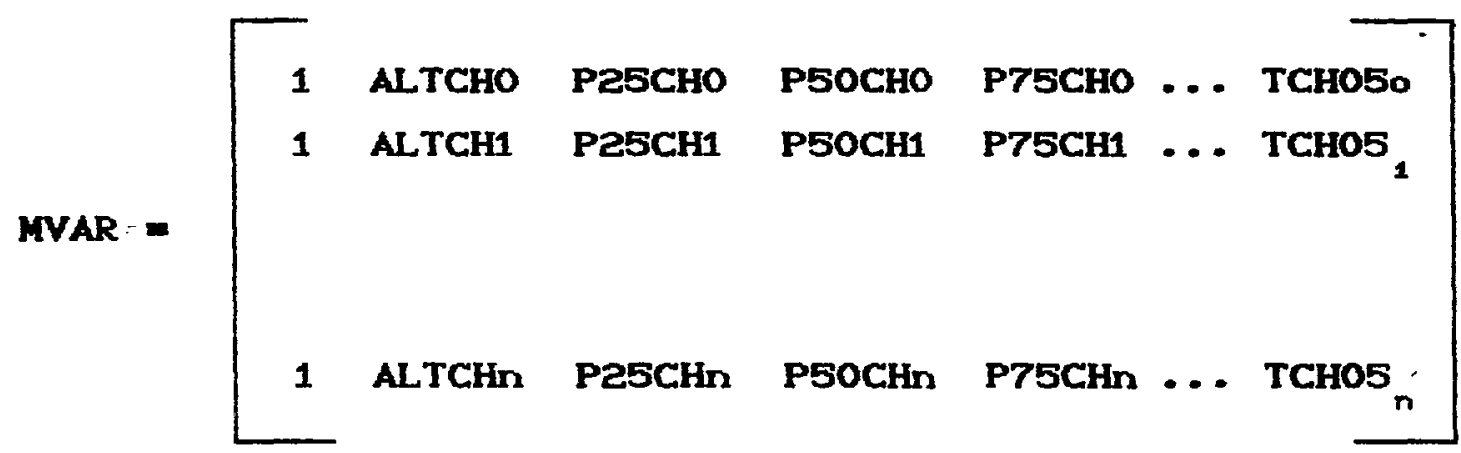

Após a estimativa do vetor de parâmetros $\Theta_{k}$ pelo metodo dos percentis obtem-se:

$$
\hat{\phi}_{k}=\text { fCALTCHO,..., AL TCHS, P2SCHO, . P1 OOCH5, . . TCHOSS }
$$


Na Figura 17 esta esquematizado 0 procedimento de teste do MSHJ e MSHA, enquanto na F1gura 18 tem-se um resumo da metodologia apresentada para os modelos MSHJ $=$ MSHA.

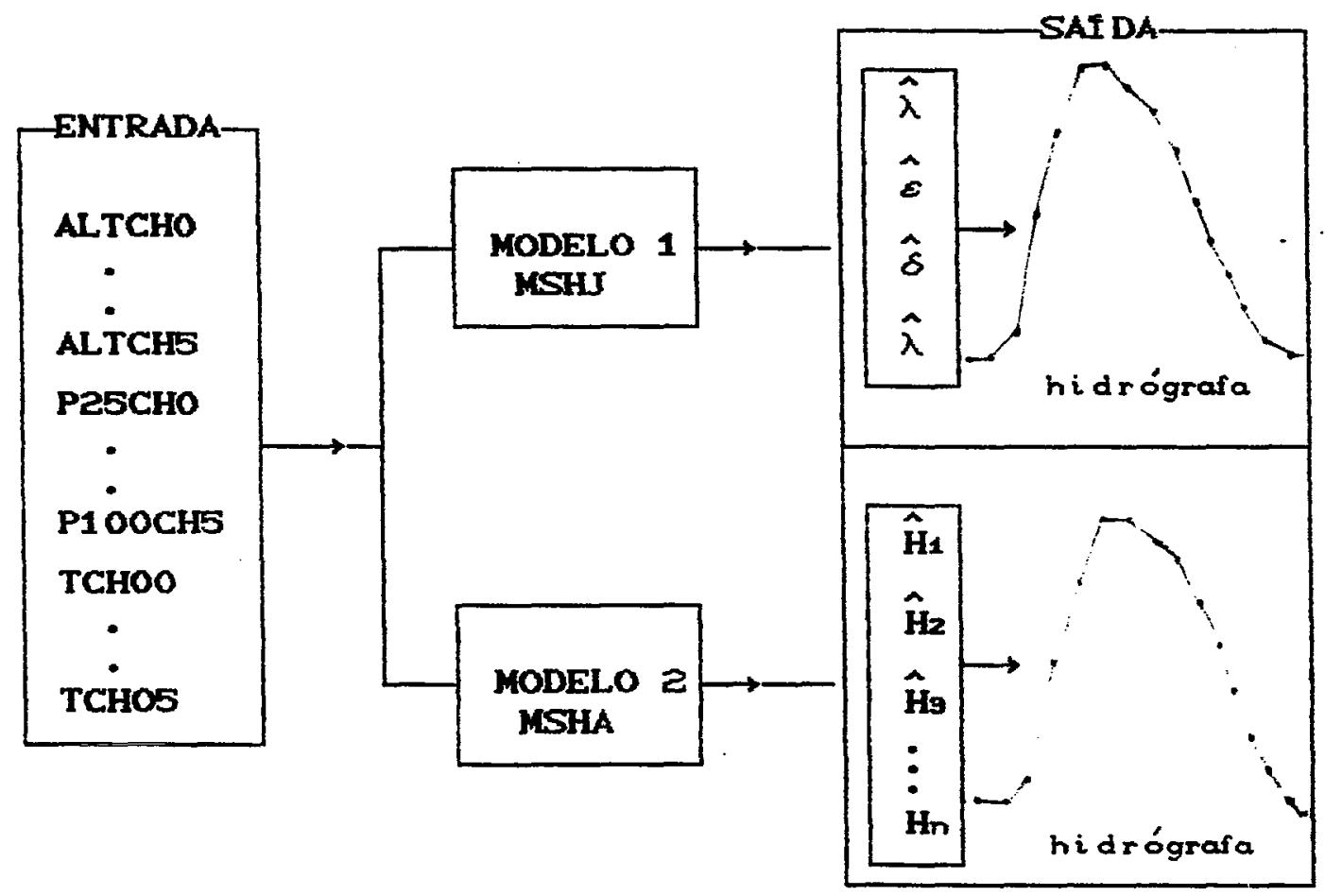

Figura 17 - Teste do modelo do modelo proposto. 


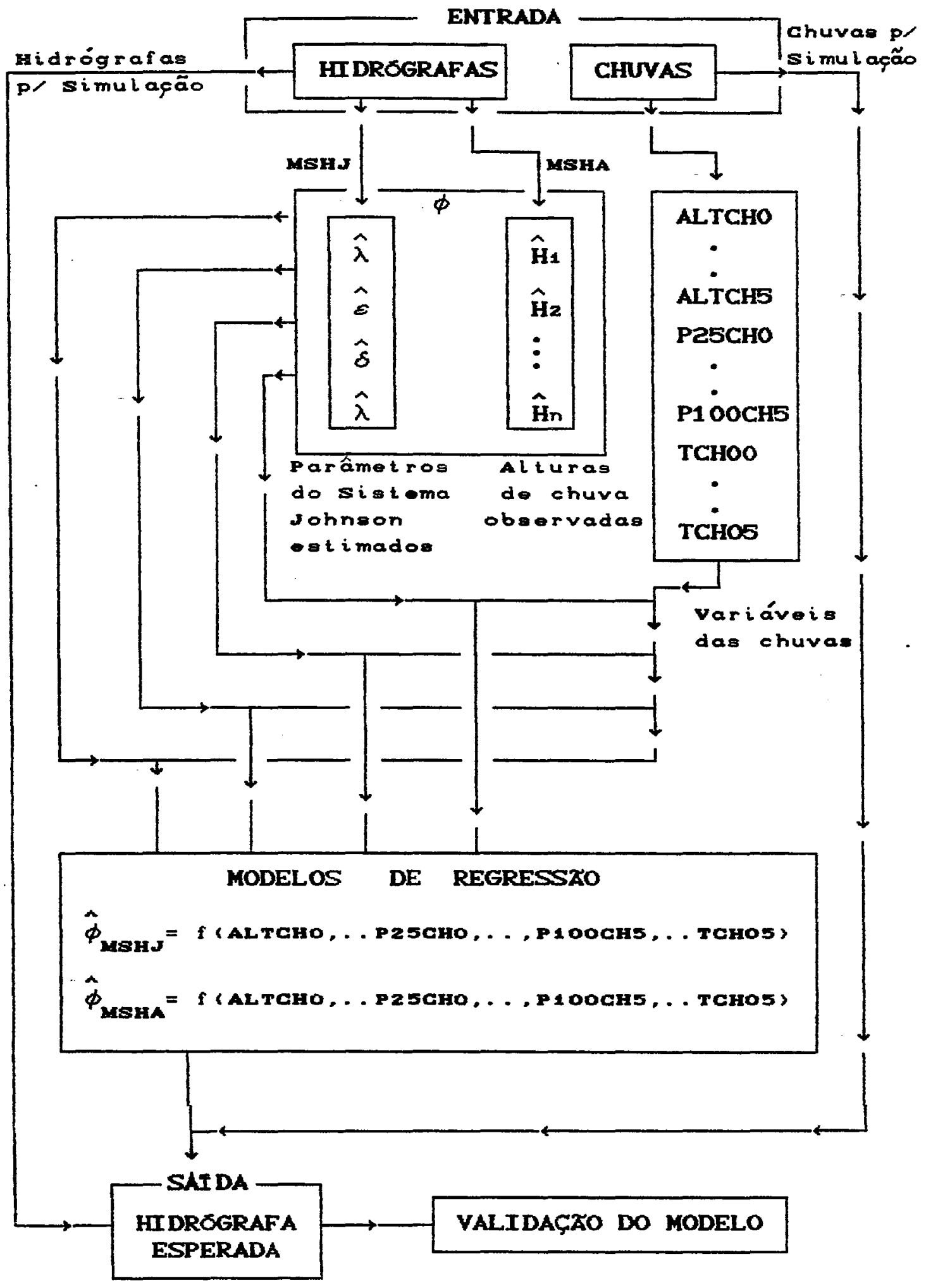

Figura 18 - Resumo da metodologia para MSHU e MSHA 


\title{
5 RESULTADOS E DISCUSSÃO
}

\author{
"O método cientifico pressupße a \\ imutabilidade dos processos \\ naturais ou do princlpio da \\ uniformidade da natureza."
}

CK. POPPER

5.1. Os dados de precipitaçăo e vazão

5.1.1. As churras

5.1.2. As hidrógrafas

5. 2. Programa de Ajuste e Simulação de Hidrógrafas - PASH

5. 2.1. Módulo "J"

5. 2.2. Módulo "A"

5.3. A Modelagem

5.3.1. MSHU - Modelos de Simulação de Hidrógrafa com base Sistema Johnson de Distribuiçăo

5.3.2. MSHA - Modelos de Simulação de Hidrógrafas com base alturas de altura de lamina d'agua

5. 4 A Simulação - Validação dos modelos 
5.1. Os dados de precipitação e vazão

A caracterização dos eventos chuvosos e das hidrografas obtidas foi feita de modo grafico, com o objetivo de apresentar, de forma resumida, as inúmeras informaçzes dos eventos existentes. Além do aspecto descritivo, a caracterização desses dados, por outro lado, visa dar uma visão parcial, das várias formas de distribuição existentes.

5.1.1. As Churas

As estatisticas referentes a Distribuição de Frequiencia das intensidades de chuva estão no Tabela 7 . Foram selecionadas duas chuvas para cada uma das estatisticas média, moda e extremos (máximos e minimos).

Observa-se a tendencia para forte assimetria positiva na Distribuição de Frequiencia representada na Figura 19 com $74.4 \%$ das chuvas com ocorrencia na primeira classe de intensidade. Esta caracteristica inicial do comportamento das variáveis que estimulam o Sistema Bacia Hidrográfica, além de caracterizar o ecossistema sob o ponto de vista hidrico, e de fundamental importancia para as análises subsequientes de model agem e simul ação. 
GAP. 5 - RESULTADOS E DISCUSSÃO

.65.

Tabela 7 - Estatistica da Distribuição das intensidades de chuva $(\mathrm{mm} / \mathrm{h})$ das chuvas atuais CHOi.

\begin{tabular}{llc}
\hline ESTATISTICAS & VALOR & NÚMERO DA CHUVA \\
\hline Observaços & 82 & - \\
Média & 5,58 & $\mathrm{CH} 3$ e CH18 \\
Desvio Padrao & 3,61 & - \\
Coef. Variaçå & $64,69 \%$ & - \\
Moda & 4,25 & $\mathrm{CHOZ}$ \\
& 4,32 & $\mathrm{CHO} 4$ \\
Mínimo & 0,78 & $\mathrm{CH} 32$ \\
& 0,90 & $\mathrm{CH} 33$ \\
Máximo & 14,50 & $\mathrm{CHO9}$ e CH67 \\
\hline
\end{tabular}

\section{DISTRIBUICACAO DAS INTENSIDADES DE CHUVA Período24/ABR/82 a 25/DEZ/83 Hacia "D"}

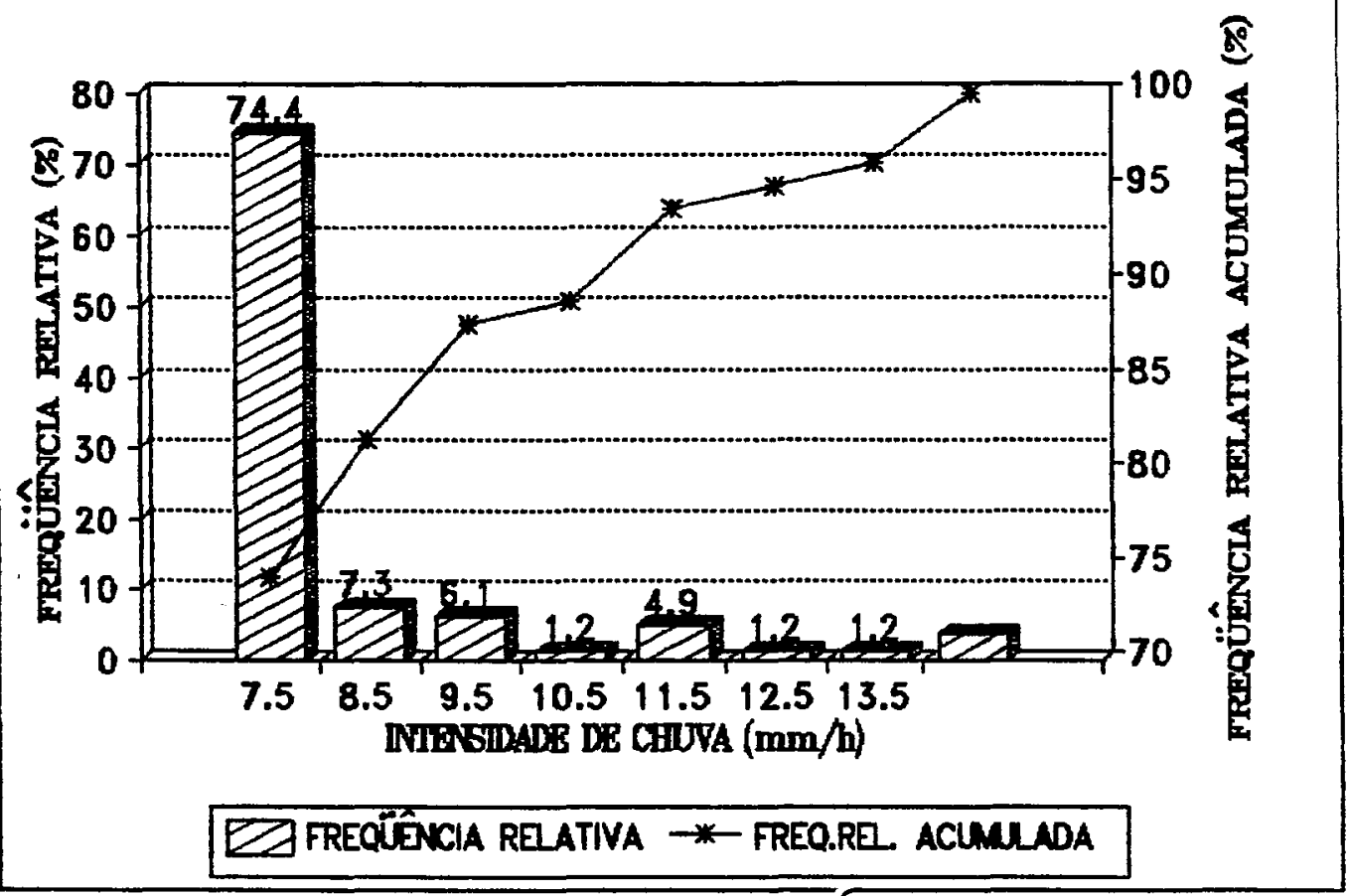

Figura 19 - Distribuição de Frequéncia das alturas de lamina d'agua das chuvas atuais da bacia experimental " $D$ ", núcleo de Cunha S.P. 
Na sequiencia, os pares de chuvas das estatisticas referidas anteriormente são representados gráficamente.

As chuvas de intensidade minima CFigura 20), tếm como caracteristica tendência a uniformidade na distribuição de frequiência dos valores de precipitaço no tempo (min). O oposto e encontrado nas distribuiços das intensidades de chuva modais (mais frequientes) e medias, Figuras 21 22. Estas se caracterizam por apresentarem descontinuidade ao longo do tempo, picos de alta intensidade e longa duração.

As chuvas de intensidade máxima se caracterizam por curto perído de duração e a tendência para intervalos de pico de chuva, como representado nas chuvas 67 e (Figura 23 ).

Os dados de campo que possibilitaram a geração destas encontram-se no APENDICE 1.

Cabe ressaltar que somente as churas atuais estão sendo apresentadas, tanto nas distribuiç̋es anteriores, como nos dados em APENDICE. Evidentemente as situaçöes em que as chuvas anteriores não são as chuvas que provocaram as vazßes escolhidas, normalmente por serem de baixa intensidade, so ocorrem em número de 10 (dez), perfazendo um total de $12,2 \%$ das chuvas atuais totais CCHOIJ, em número de 82. 


\section{CHUVA DE INTENSIDADE MINIMA $(0.78 \mathrm{~mm} / \mathrm{h})$ Data : 20/mar/83 Hora:14h50' Chuva 32}

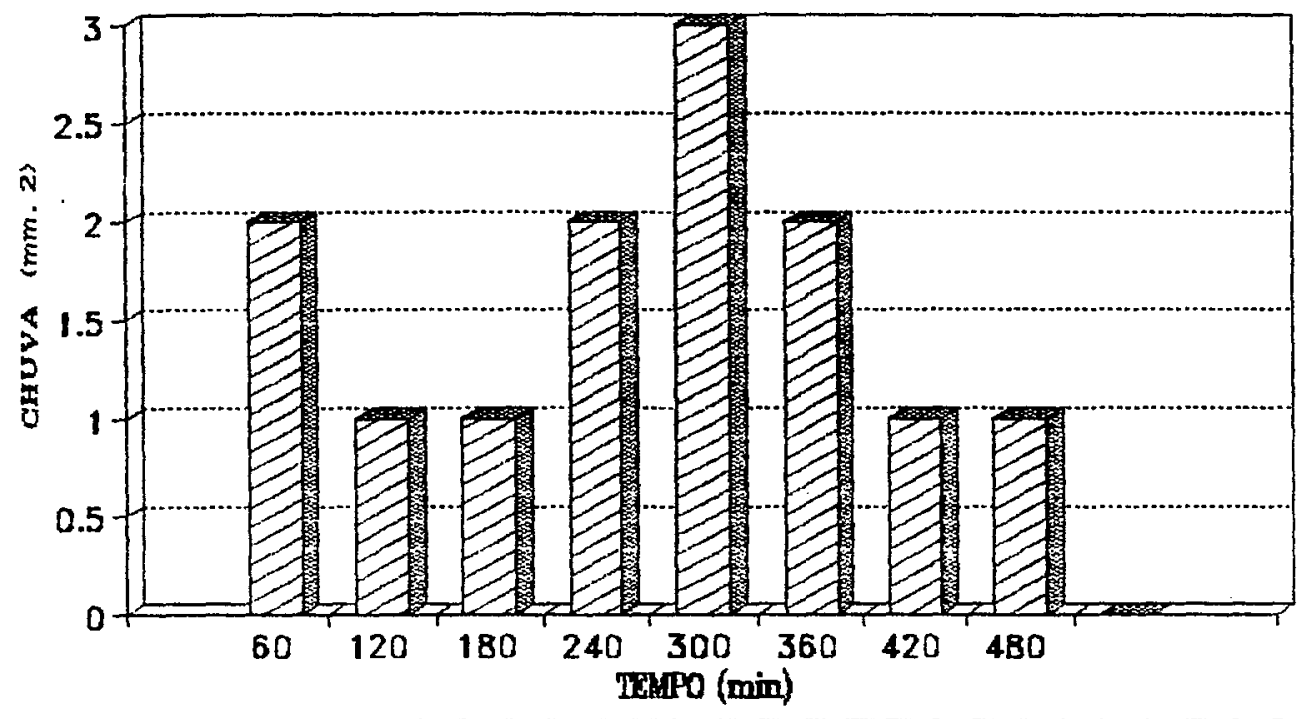

\section{CHUVA DE INTENSIDADE MÍNIMA $(0.9 \mathrm{~mm} / \mathrm{h})$ Data : 22/mar/83 Hora:15h30' Chuva 33}

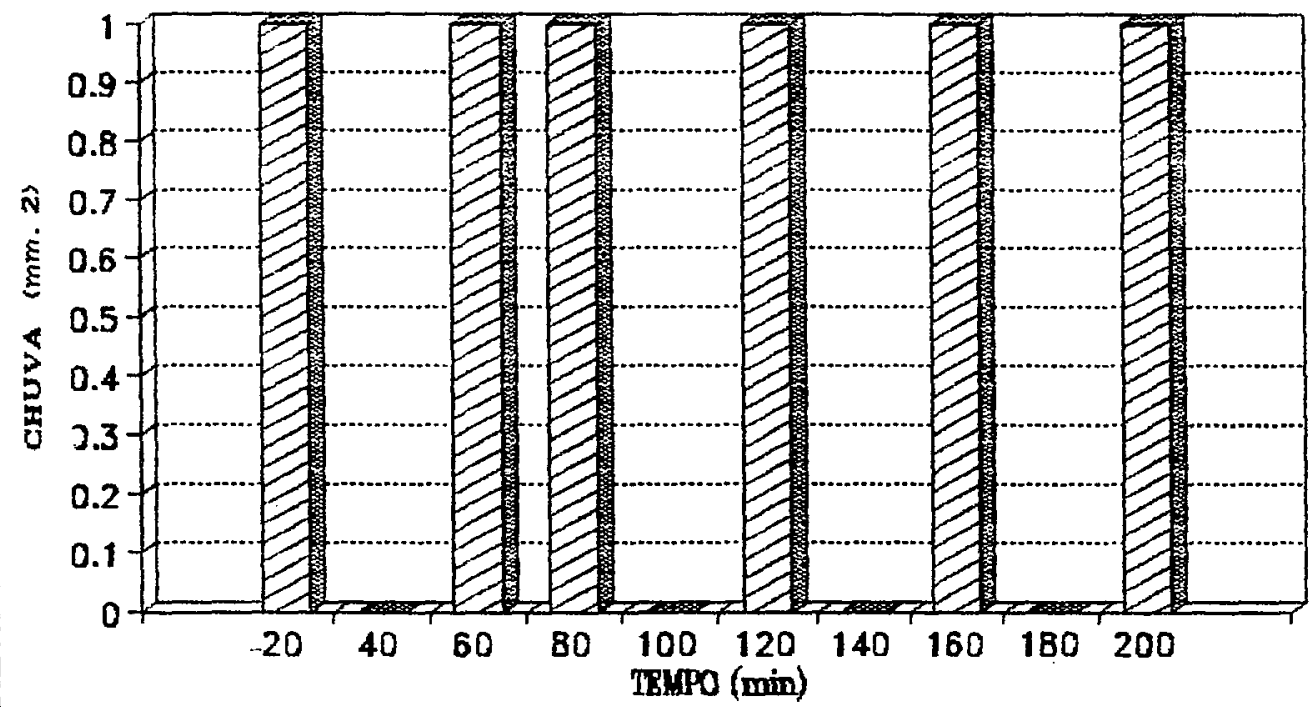

Figura 2o - Distribuiçőes das chuvas de altura minima da Bacia de Cunha S.P. Bacia de Cunha S.P. 


\section{CHUVA DE INTENSIDADE MODAL $(4.25 \mathrm{~mm} / \mathrm{h})$ Data : 22/JN/82 Hora/h20' Chuva 2}

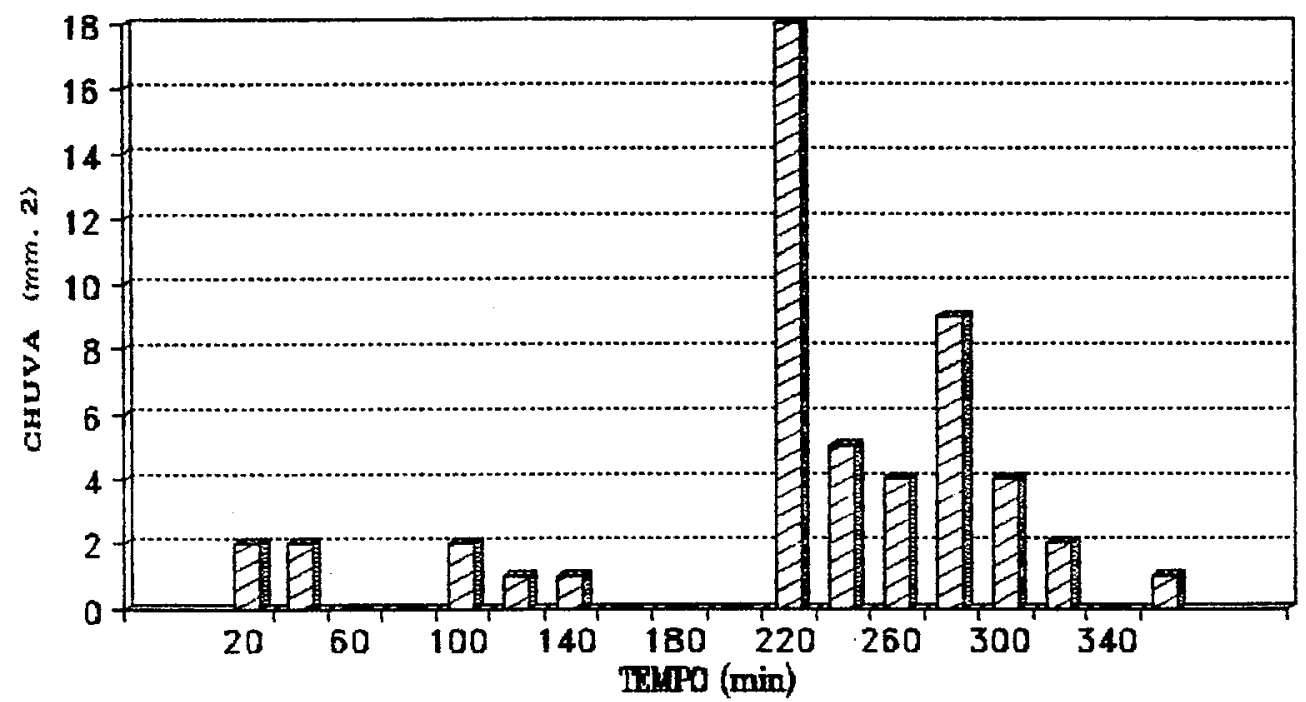

CHUVA DE INTENSIDADE MODAL $(4.32 \mathrm{~mm} / \mathrm{h})$ Data : 25/JUN/82 Hora:0h20' Chuva 4

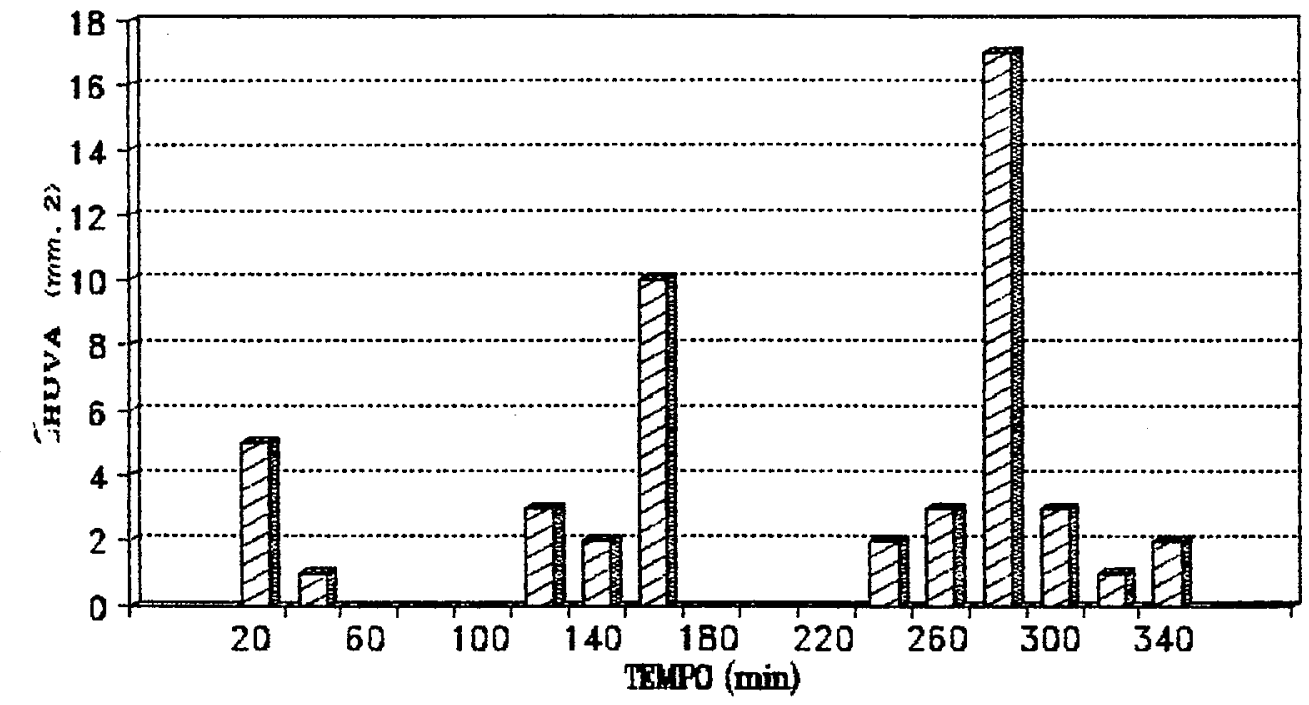

Figura 21 - Distribuiçros das chuvas de altura modal da Bacia de Cunha S.P. 


\section{CHUVA DE INTENSIDADE MÉDIA $(5.35 \mathrm{~mm} / \mathrm{h})$}

Data : 30/DEZ/82 Hora:8h20' Chuva 13

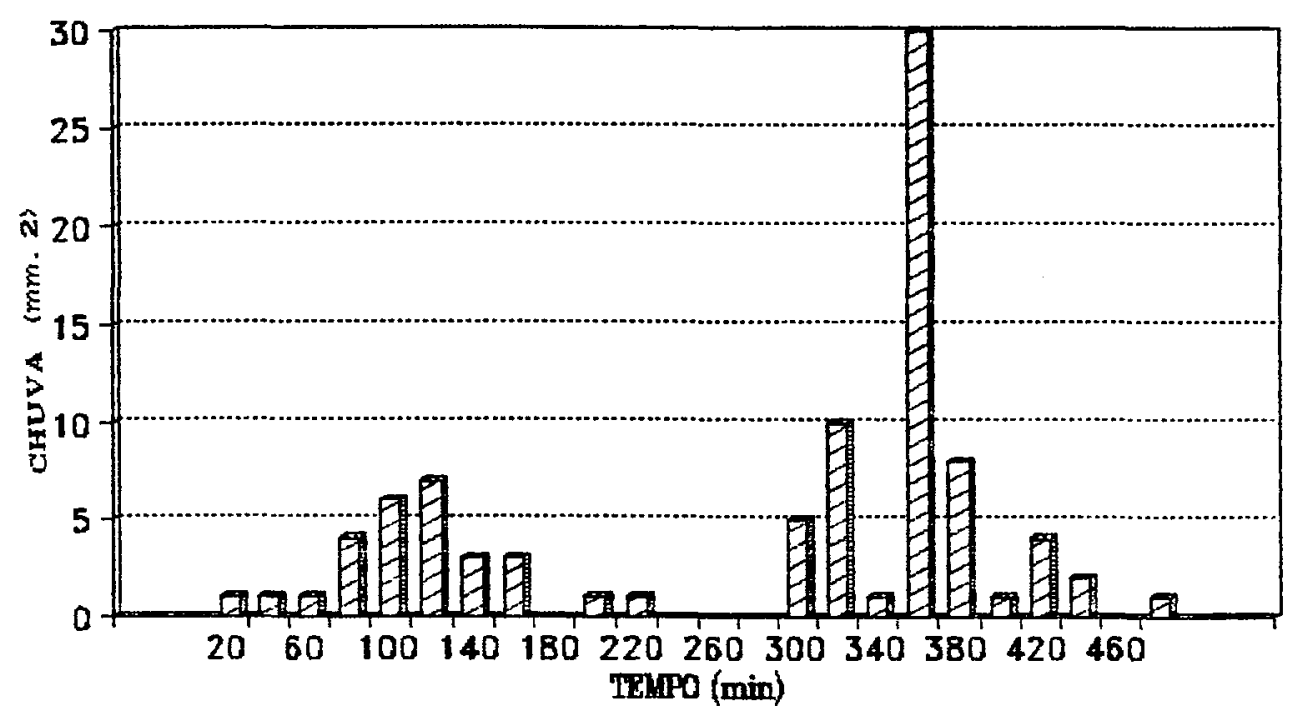

\section{CHUVA DE INTENSIDADE MÉDIA $(5.35 \mathrm{~mm} / \mathrm{h})$ Data : 12/JAN/82 Hora:14h10' Chuva 18}

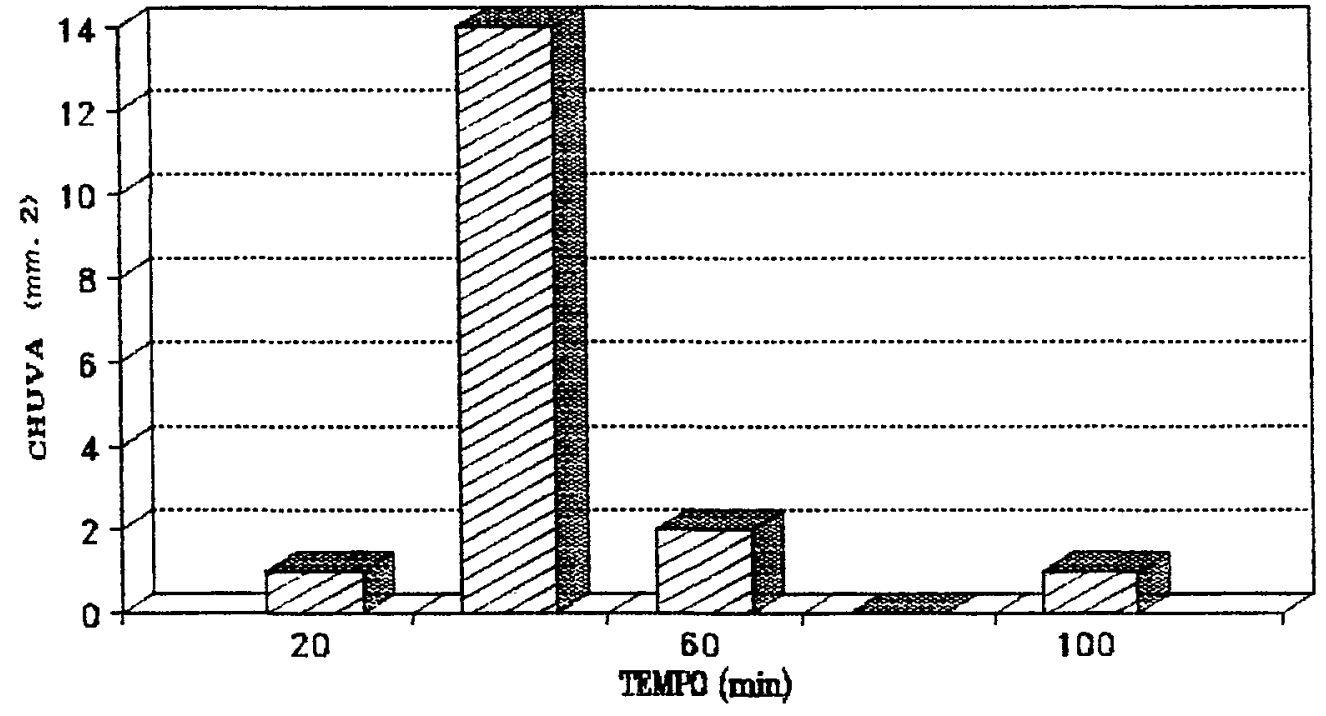

Figura 22 - Distribuiçóes das chuvas de altura média da Bacia de Cunha S.P. 


\section{CHUVA DE INTENSTDADE MÁXIMA $(14.5 \mathrm{~mm} / \mathrm{h})$} Data : 16/NOV/82 Horafh50' Chuva 9

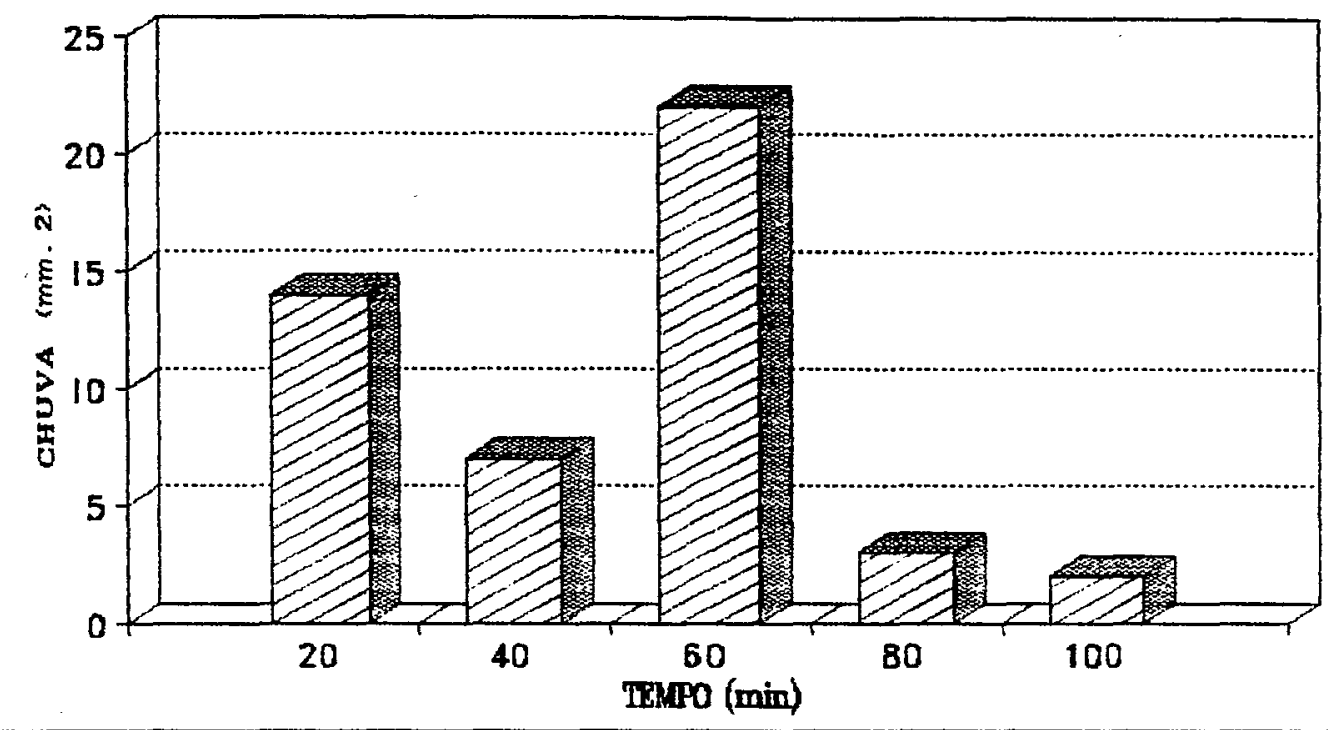

\section{CHUVA DE INTENSIDADE MÁXIMA $(14.5 \mathrm{~mm} / \mathrm{h})$} Data : 19/NOV/82 Hora:23h15' Chuva 67

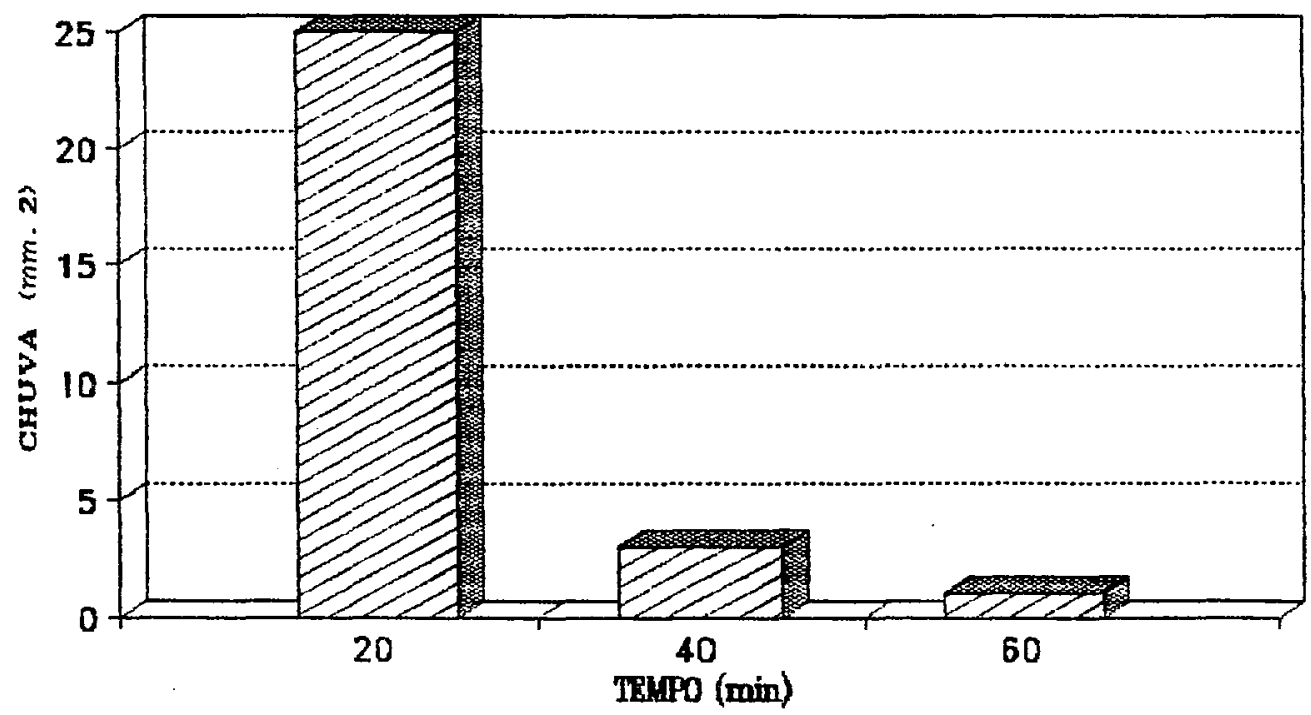

Figura 23 - Distribuiçơs das chuvas de altura máxima da Bacia de Cunha S.P. 


\subsubsection{As Hidrógrafas}

As estatisticas referentes a Distribuição de Frequiencia das vazôes das hidrógrafas estão no Tabela 8. Da mesma forma foram selecionadas duas hidrógrafas para cada uma das estatisticas média, modal e extremos cmáximos e mínimoss.

A distribuição das vazరes mantém assimetria positiva com menor intensidade, em relação à distribuição das intensiadades de chuva, podendo-se atingir os $70 \%$ dos dados (Freqéncia Acumulada) na quarta classe de vazão, já que para as intensidades de chuva apresentava-se na primeira classe (Figura 24).

Esta diferença de padrão de distribuição com $39 \%$ para a primeira classe e $30 \%$ para a segunda, pode indicar a importáncia das chuvas anteriores mais próximas a chuva atual na composição das hidrógrafas.

As hidrógrafas de vazão minima, (Figura 25). apresentam padrao bastante caracteristico, observando-se intervalos de tempo com vazão constante. Estas hidrografas sáo resultantes de chuvas de baixa intensidades e uniformidade caracteristica, assemel hando-se a hidrogramas de bacias impermeáveis quando também estimuladas por chuva similares, (LIMA 1986 ).

Tanto as hidrógrafas de vazão modal, media e maxima (Figuras 26 a 28 ) apresentam padrós de assimetria positiva, evidentemente existindo exceçóes. 
Tabela 8 - Estatistica da Distribuição das intensidades de vazão $(\mathrm{cm} / \mathrm{h})$ das hidrógrafas modeladas.

\begin{tabular}{llc}
\hline ESTATISTICAS & VALOR & NUMERO DA CHUVA \\
\hline Observaç̃̇es & 82 & - \\
Media & 5,9 & CHOS e CH55 \\
Desvio Padrão & 4,7 & - \\
Coef. Variação & $79,2 \%$ & - \\
Moda & 2,6 & CHO2 e CHOS \\
Minimo & 1,4 & CH32 e CH46 \\
Máximo & 18,1 & CH38 \\
& 18,2 & CH59
\end{tabular}

\section{DISTRIBUTCÃ̃O DE VAZÃ0 DAS HIDRÓGRAFAS} Periodo:24/ABR/82 a 25/dez/83 Bacia "D"

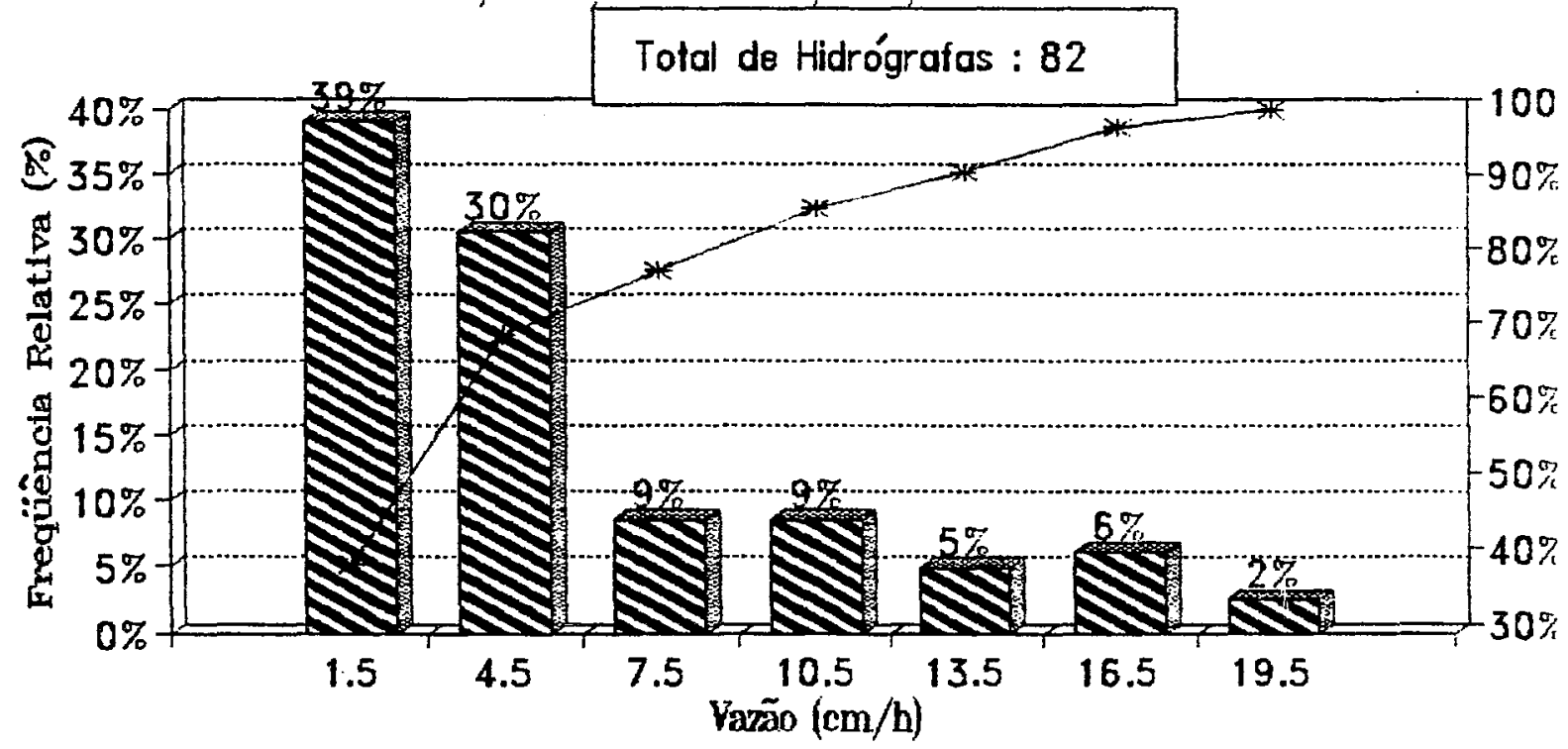

Freqüência Relativa * Freq.Rel. Acumulada

Figura 24 - Distribuição de Frequiência das alturas de lámina d'agua das vazóes das hidrogrfas, bacia experimental "D", núcleo de Cunha S.P. 

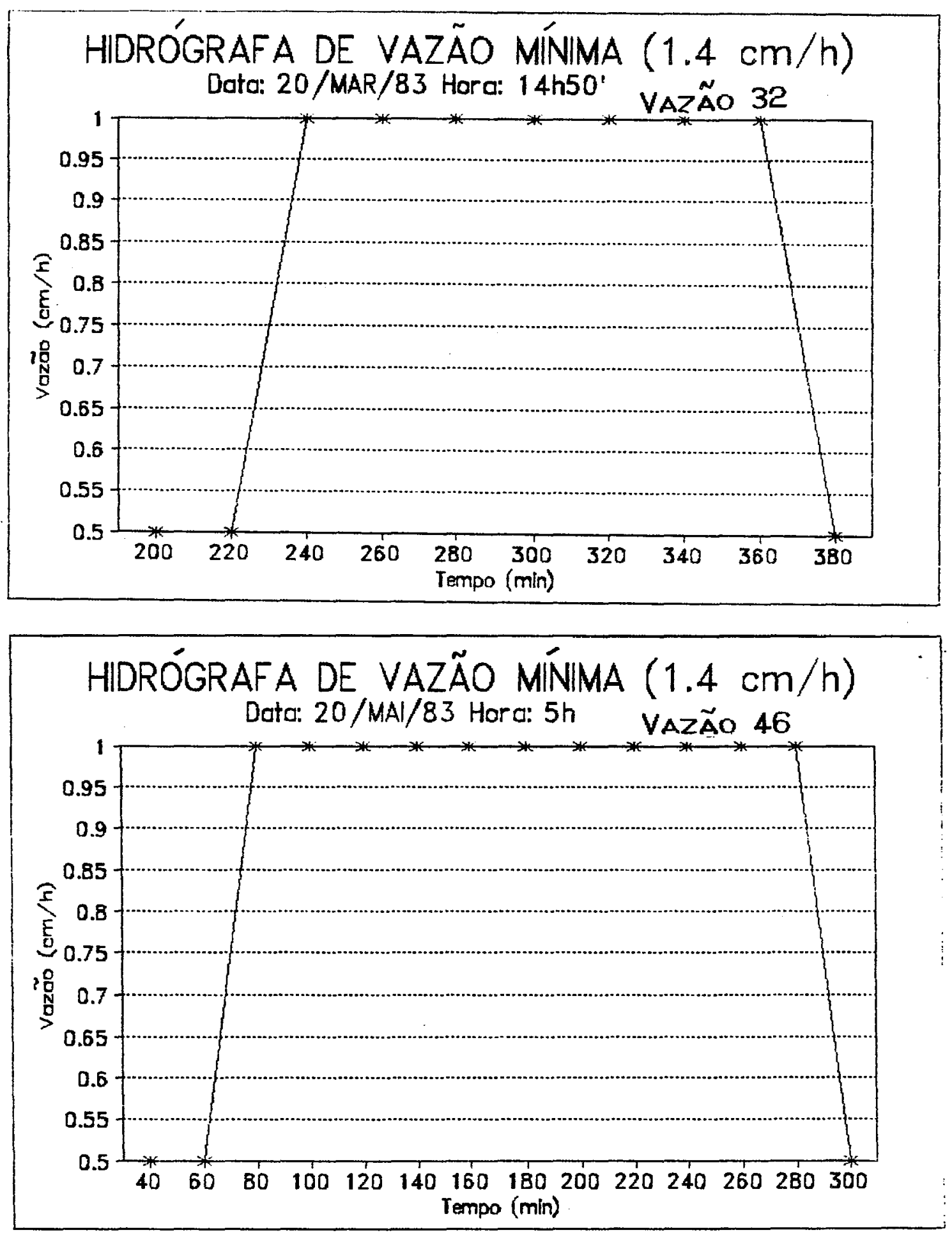

Figura 25 - Distribuiçós das vazóes minimas das Hidrografas na Bacia de Cunha S.P. 

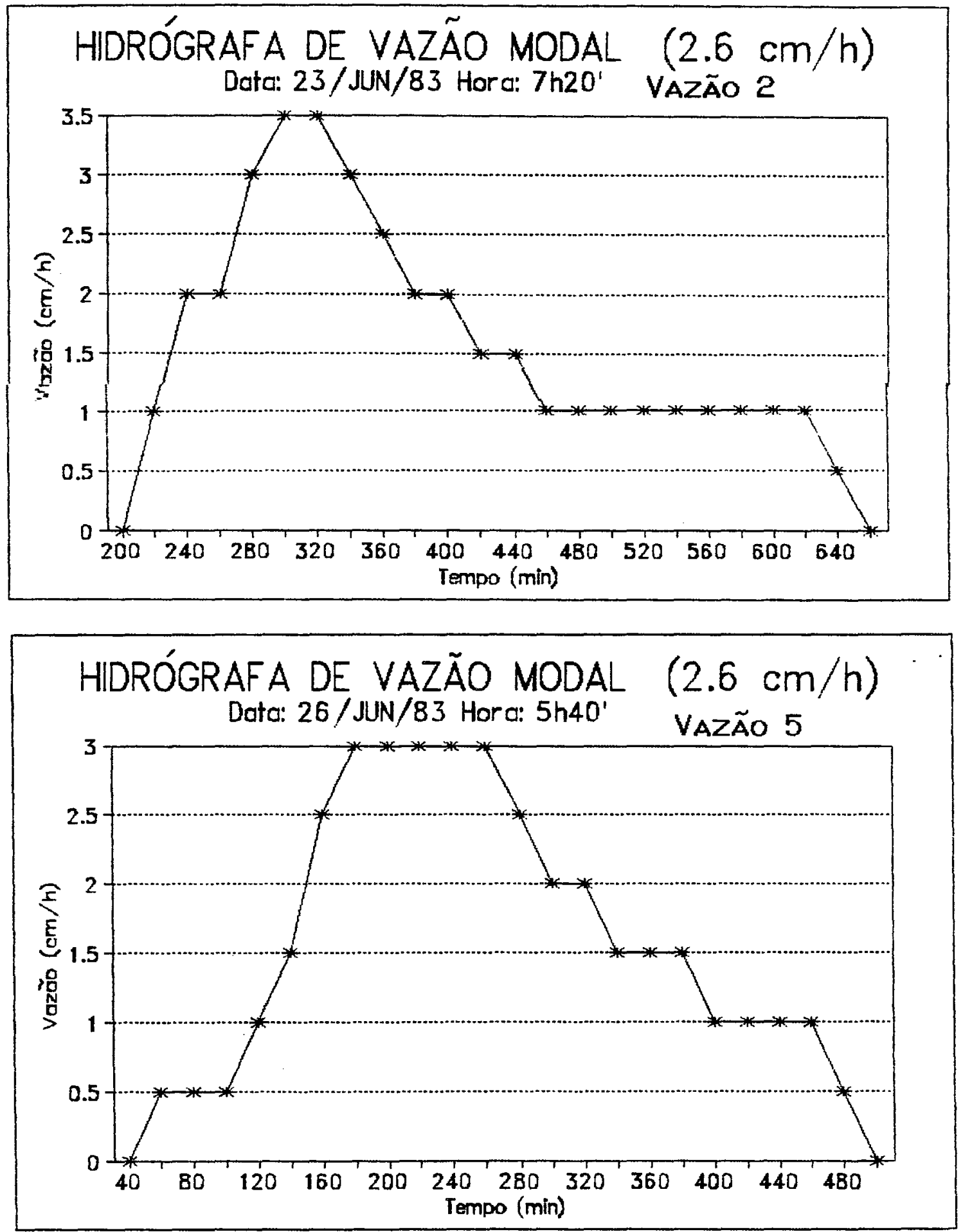

Figura 26 - Distribuiçơes das vazões modais das Hidrografas na Bacia de Cunha S.P. 

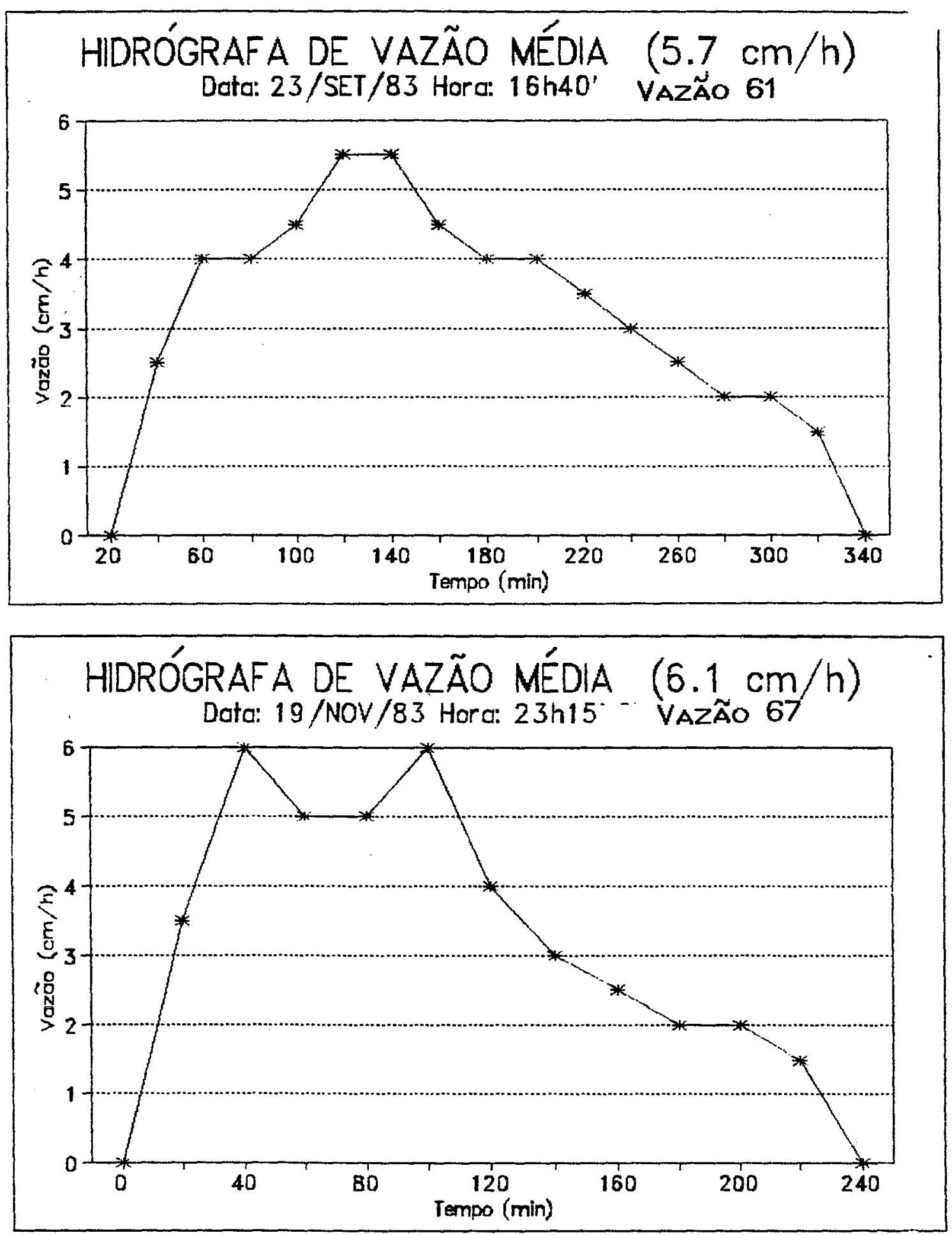

Figura 27 - Distribuiç̋es das vazơes médias das Hidrografas na Bacia de Cunha S.P. 

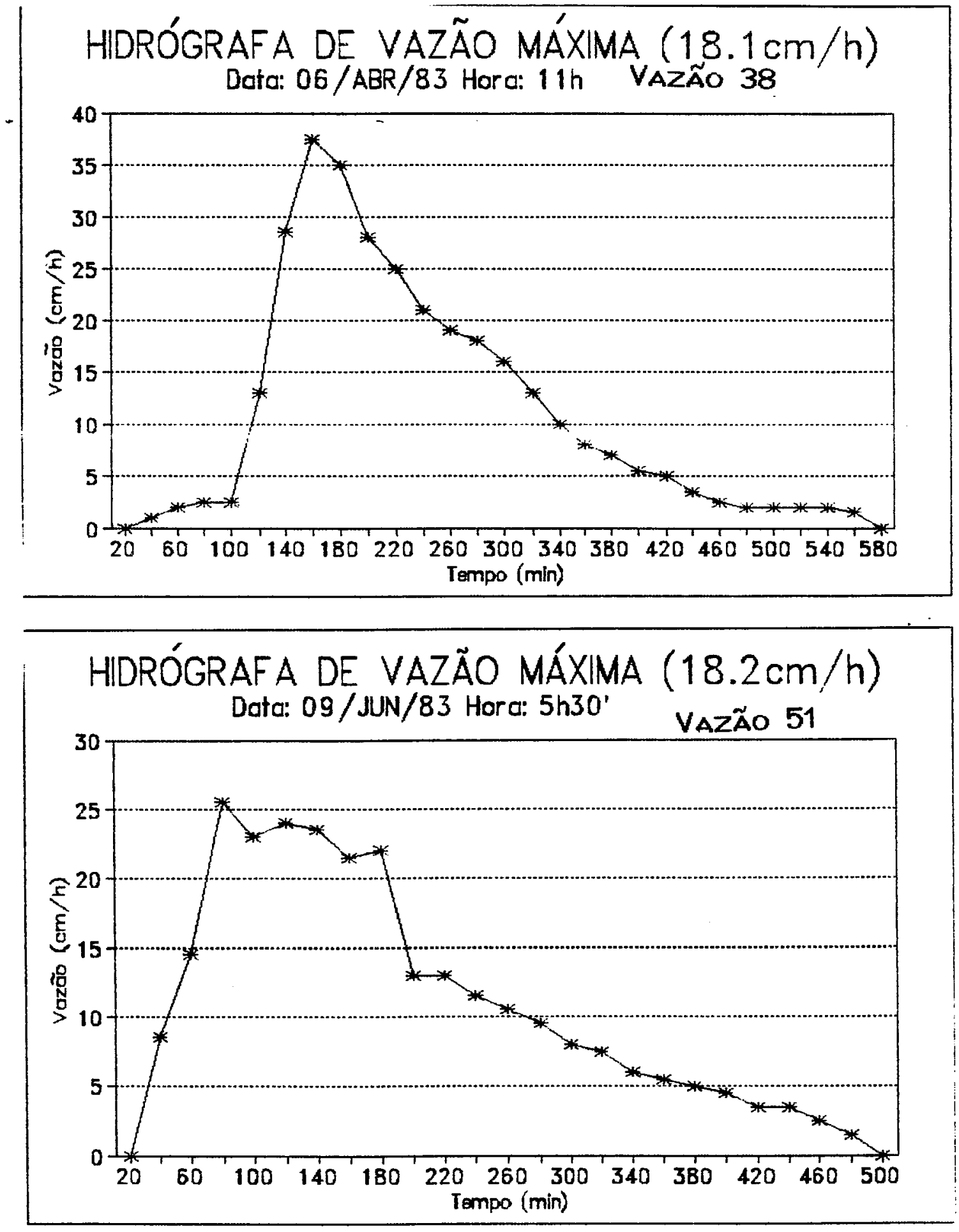

Figura 28 - Distribuiçర̃es das vazర̃es máximas das Hidrógrafas na Bacia de Cunha S.P. 
5.2. Programa de Ajuste e Simulação de Hidrógrafas - PASH

Como visto no capitulo III referente ao Sistema Jonhson de Distribuição, a manipulação das expresşes para estimativa dos parámetros pelo método dos percentis, se tornaria bastante complicada caso não se pudesse contar com ajuda da informática para a computação dos dados.

Outro aspecto importante está relacionado ao volume de dados existentes, como demonstrado pelo elevado número de hidrógrafas e principalmente de chuvas utilizadas para gerar os parâmetros, como também a manipulação das matrizes para a estimativa dos coeficientes de regressão dos modelos sugeridos (MSHJ e MSHA).

Foi elaborado um programa CPASH, em linguagem SAS, CSTATISTICAL ANALYSIS SYSTEM INSTITUTE, 1985a; STATISTICAL ANALYSIS SYSTEM INSTITUTE, $1985 \mathrm{~b} 3$. versão 5, instalado num computador IBM 3090 modelo 150 (32Mb de memória real), 23Gb de área em disco, pertencente a DATAMIL, empresa coligada à Cia. Suzano de Papel e Cel ul ose.

- PASH CAPENDICE 4) tem como objetivo efetuar a modelagem (ajuste) e simulação das hidrógrafas existentes. Contém dois módulos principais : módulo - "J", referente ao MSHJ, e módulo "A" referente ao MSHA.

5.2.1. Módulo "J" -

Este módulo contém as seguintes etapas :

$1)$ Leitura dos dados de chuva e vazão provenientes da microbacia experimental.

2) Determinação dos Parametros das chuvas Caltura de chuva, tempos entre chuvas e percentis?.

3) Para cada hidrógrafa, geração de 100 (cem) ajustes de vazbes, utilizando-se o método do Sistema Johnson de Distribuição. Posteriormente foi feito a seleção 
do mel hor

ajuste

utilizando-se

o

teste

de

Kol mogorov-Smi rnov.

4) Análise de Regressão utilizando-se do processo "stepwise/forward", para obtenção das melhores equaçres, correlacionando as variavés independentes obtidas no item 2 , com as variáveis dependentes geradas no item 3 .

5) Estudo de Residuo e multicolinearidade, como fatores discriminantes na escolha do conjunto de equaçós representantes do modelo proposto.

6) Simulação das hidrografas (82), que serviram de base para o ajuste do modelo proposto, utilizando-se as chuvas como condiçöes antecedentes a cada evento consi derado.

5.2. 2. Módulo "A"

Este módulo contém as seguintes etapas :

1) Leitura dos dados de Chuva e Vazão provenientes da, microbacia experimental.

2) Determinação dos Parâmetros das chuvas (altura de chuva, tempos entre chuvas e percentis).

3) Análise de Regressão utilizando-se do processo "stepwise/forward", para obtenção das melhores equaçర̈es, correlacionando as variavéis independentes obtidas no item 2 , com as variáveis dependentes geradas no item 1 Calturas de lâmina d'água).

5) Estudo do Residuo como fator discriminante na escolha do conjunto de equações representantes do modelo proposto.

6) Simulação das hidrógrafas (82), que serviram de base para o ajuste do modelo proposto. utilizando-se as chuvas como condiçäes antecedentes a cada evento considerado.

Nas Tabelas $\theta+10$ são apresentadas as estatisticas relativas as variáveis das chuvas Centradas do 
sistemas, utilizadas nos modelos de simul ação.

As variáveis da chuva são os primeiros produtos do PASH, sendo então numa fase posterior utilizadas nos processos de correlação e regressão com as variáveis de saida do Sistema Bacia Hidrográfica.

- padrão exponencial de Distribuição de Frequiencia é generalizado entre as variáveis da chuva, caracterizado por forte assimetria positiva, com a mediana CD) posicionada entre a média $(\bar{X})$ a a moda $(M D$, isto é :

$$
M<D<X
$$

e com o terceiro momento em relação à média. coeficente de assimetria (m3) positivo; mantendo concordância com as distribuiçőes intensidade de chuva e vazão apresentadas anteriormente. 


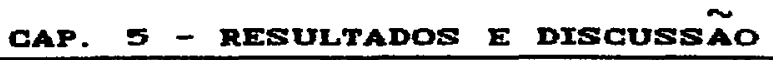

Tabela $\theta$ - Momentos das variáveis da chuva - CHUVAR

\begin{tabular}{|c|c|c|c|c|c|}
\hline CHUVAR & $\mathbf{n}$ & $\mathbf{X}_{\mathbf{m}}$ & $\mathbf{S}$ & $M$ & $\mathrm{CV}$ \\
\hline TCHOD & 81 & 0.00 & 0.00 & 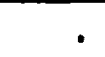 & . \\
\hline FICHD & 81 & 17.37 & 11.39 & 0.92 & 65.59 \\
\hline P25CHD & 81 & 0.85 & 0.93 & 1.52 & 97.19 \\
\hline P50CHD & 81 & 1.59 & 1.50 & 1.50 & 94.53 \\
\hline P75CHD & 81 & 2.33 & 1.97 & 1.40 & 84.44 \\
\hline P100CHD & 81 & 3.97 & 2.88 & 1.45 & 72.66 \\
\hline TCHD1 & 81 & 27.84 & 32.18 & 1.70 & 115.59 \\
\hline VxcH1 & 81 & 12.25 & 12.22 & 1.14 & 99.73 \\
\hline P25CH1 & 81 & 0.71 & 0.94 & 1.65 & 131.39 \\
\hline P50CH1 & 81 & 1.17 & 1.40 & 1.64 & 125.38 \\
\hline P75CH1 & 81 & 1.64 & 1.83 & 1.18 & 11.15 \\
\hline P100CH1 & 81 & 2.71 & 2.78 & 1.20 & 102.50 \\
\hline TCHD2 & 81 & 39.84 & 57.07 & 3.33 & 143.25 \\
\hline WICHR & 81 & 8.15 & 11.63 & 1.99 & 142.57 \\
\hline P25CHR & 81 & 0.53 & 0.88 & 2.35 & 163.98 \\
\hline P50CHR & 81 & 0.83 & 1.23 & 1.79 & 148.13 \\
\hline P75CHR & 81 & 1.24 & 1.73 & 1.39 & 139.08 \\
\hline P100CHR & 81 & 2.02 & 2.70 & 1.37 & 133.16 \\
\hline TCHB3 & 81 & 28.01 & 44.34 & 1.21 & 158.30 \\
\hline VOCHB & 81 & $\mathbf{3 . 6 7}$ & 7.13 & 2.32 & 194.44 \\
\hline P25CH3 & 81 & 0.25 & 0.61 & 3.51 & 249.59 \\
\hline P50CA3 & 81 & 0.35 & 0.80 & 1.15 & 224.78 \\
\hline Р75CHB & 81 & 0.63 & 1.29 & 2.35 & 204.57 \\
\hline P100CA3 & 81 & 0.97 & 1.93 & 2.35 & 198.60 \\
\hline TCHD4 & 81 & 16.44 & 37.59 & 2.04 & 228.67 \\
\hline vich4 & 81 & 2.30 & 6.46 & 3.38 & 281.32 \\
\hline P25CH4 & 81 & 0.14 & 0.46 & 4.30 & 332.40 \\
\hline P50CH4 & 81 & 0.22 & 0.69 & 4.15 & 313.66 \\
\hline P75CH4 & 81 & 0.37 & 1.21 & 4.50 & 323.58 \\
\hline P100CH4 & 81 & 0.56 & 1.63 & 4.01 & 291.91 \\
\hline TCHD5 & 81 & 10.63 & 33.27 & 3.04 & 313.04 \\
\hline YOLCE5 & 81 & 0.87 & 3.76 & 6.21 & 432.26 \\
\hline P25CH5 & 81 & 0.07 & 0.31 & 5.91 & 428.61 \\
\hline P50CH5 & 81 & 0.12 & 0.45 & 4.51 & 378.31 \\
\hline P75CH5 & 81 & 0.17 & 0.62 & 4.19 & 367.98 \\
\hline P100CH5 & 81 & 0.22 & 0.75 & 3.77 & 346.12 \\
\hline
\end{tabular}

$n=n u ́ m e r o$ de observações; $X m=m e d i a ; \quad S=d e s v i o$ padrão; M3=coeficiente de assimetria; CV=coeficiente de variação. 
GAP. 5 - RESULTADOS E DISCUSSÃO

Tabela 10 - Quartis das variáveis da chuva - CHUVAR

\begin{tabular}{|c|c|c|c|c|c|c|}
\hline CHUVAR & $\begin{array}{r}\text { MTIID } \\
0 \%\end{array}$ & 0.25 & $\begin{array}{r}\text { WDIANA } \\
0.50 \\
\end{array}$ & 0.75 & $\begin{array}{r}\text { YAXITO } \\
1.00 \\
\end{array}$ & MODA \\
\hline TCHDO & 0.00 & 0.00 & 0.00 & 0.00 & 0.00 & 0.00 \\
\hline vach & 3.00 & 8.25 & 13.50 & 25.25 & 52.00 & 6.50 \\
\hline P25CHD & 0.09 & 0.33 & 0.56 & 1.41 & 4.21 & 0.42 \\
\hline P50CHD & 0.18 & 0.54 & 0.96 & 2.30 & 7.08 & 4.17 \\
\hline P75CHD & 0.27 & 0.81 & 1.63 & 3.25 & 9.62 & 3.25 \\
\hline P100CHD & 0.67 & 2.00 & 3.00 & 5.17 & 14.67 & 3.00 \\
\hline $\mathrm{TCED1}$ & 0.00 & 2.53 & 21.78 & 35.49 & 137.78 & 0.00 \\
\hline vach1 & 0.00 & 2.25 & 9.00 & 18.50 & 52.00 & 0.00 \\
\hline P25CH1 & 0.00 & 0.09 & 0.31 & 0.91 & 3.91 & 0.00 \\
\hline P51CH1 & 0.00 & 0.19 & 0.54 & 1.51 & 5.68 & 0.00 \\
\hline P75CH1 & 0.00 & 0.28 & 0.99 & 2.50 & 6.38 & 0.00 \\
\hline P100CH1 & 0.00 & 0.68 & 2.00 & $\mathbf{3 . 8 3}$ & 11.33 & 0.00 \\
\hline TCHD2 & 0.00 & 0.00 & 25.05 & B6.02 & 397.82 & 0.00 \\
\hline VoLCH2 & 0.00 & 0.00 & 3.00 & 12.25 & 61.50 & 0.00 \\
\hline P25CH2 & 0.00 & 0.00 & 0.13 & 0.71 & 4.65 & 0.00 \\
\hline P52CH2 & 0.00 & 0.00 & 0.27 & 1.29 & 5.42 & 0.00 \\
\hline P75CHR & 0.00 & 0.00 & 0.51 & 1.70 & 5.75 & 0.00 \\
\hline P100CHE & 0.00 & 0.00 & 1.00 & 3.16 & 9.87 & 0.00 \\
\hline TCH3 & 0.00 & 0.00 & 0.00 & 52.86 & 138.97 & 0.00 \\
\hline VICHB & 0.00 & 0.00 & 0.00 & 4.75 & 32.50 & 0.00 \\
\hline P25CH3 & 0.00 & 0.00 & 0.00 & 0.22 & 3.42 & 0.00 \\
\hline P50CH3 & 0.00 & 0.00 & 0.00 & 0.47 & 4.17 & 0.00 \\
\hline P75CH3 & 0.00 & 0.00 & 0.00 & 0.60 & 5.25 & 0.00 \\
\hline P100CH & 0.00 & 0.00 & 0.00 & 1.00 & 8.68 & 0.00 \\
\hline TCHO4 & 0.00 & 0.00 & 0.00 & 0.00 & 130.58 & 0.00 \\
\hline VICH4 & 0.00 & 0.00 & 0.00 & 0.00 & 32.50 & 0.00 \\
\hline P25CH4 & 0.00 & 0.00 & 0.00 & 0.00 & 2.67 & 0.00 \\
\hline P50CH4 & 0.00 & 0.00 & 0.00 & 0.00 & 4.33 & 0.00 \\
\hline P75CH4 & 0.00 & 0.00 & 0.00 & 0.00 & 8.00 & 0.00 \\
\hline P100CH4 & 0.00 & 0.00 & 0.00 & 0.00 & 9.67 & 0.00 \\
\hline TCHD5 & 0.00 & 0.00 & 0.00 & 0.00 & 150.65 & 0.00 \\
\hline VICH5 & 0.00 & 0.00 & 0.00 & 0.00 & 29.50 & 0.00 \\
\hline Р25СН5 & 0.00 & 0.00 & 0.00 & 0.00 & 2.35 & 0.00 \\
\hline P50СH5 & 0.00 & 0.00 & 0.00 & 0.00 & 2.67 & 0.00 \\
\hline P75CH5 & 0.00 & 0.00 & 0.00 & 0.00 & 3.67 & 0.00 \\
\hline P100CH5 & 0.00 & 0.00 & 0.00 & 0.00 & 4.33 & 0.00 \\
\hline
\end{tabular}


- segundo produto do PASH 6 o vetor de parámetros obtido por estimativa, caracterizando e representando as hidrógrafas escolhidas para a simulação.

Os parámetros de localização lambda $(\lambda)$ e epsilon ( 2 ), foram determinados, e não estimados, sendo então obtidos dos dados brutos iniciais do campo. Por serem de fácil determinação nas distribuiçóes de vazão, este caminho pode ser seguido. Facilitou-se o estudo futuro de modelagem e simulação, e principalmente foi eliminado o efeito aleatório do erro (ei), existentes em modelos de regressão.

No entanto já os parámetros ligados a forma e escala do Sistema Johnson de Distribuição, gama $(\gamma)$ e delta ( 8 ), foram estimados via método dos percentis.

As Tabelas 11 e 12 apresentam, as estatisticas dos parámetros do Sistema Johnson de Distribuição de Probabilidade.

Observa-se que para a a moda CM tem o mesmo valor da mediana $C D J$ e ambos inferiores à média $(\bar{X})$. Já $\lambda$ apresenta tendéncia maior tendencia à normalidade que os outros parâmetros.

Finalmente são apresentadas as estatisticas das vazóes observadas no microbacia " $D$ ". Forte assimetria também é encontrada, indicando distribuição exponencial, e podendo ser um fator de relevante no quando da análise de comportamento dos modelos MSHJ e MSHA. 
CAP. S - RESULTADOS E DISCUSSÃO

Tabela 11 - Quartis das variáveis da hidrografa - HIDVAR

\begin{tabular}{|c|c|c|c|c|c|c|}
\hline & MTIID & & MDDANA & & MaXI'Co & SODA \\
\hline HIIDYAR & $0 \%$ & $25 \%$ & $50 \%$ & $75 \%$ & $100 \%$ & \\
\hline DPSION & 20.00 & 20.00 & 40.00 & 80.00 & 280.00 & 40.00 \\
\hline FAYBDA & 100.00 & 280.00 & 380.00 & 500.00 & 1360.00 & 240.00 \\
\hline DERA & 0.26 & 0.49 & 1.14 & 2.17 & 6.16 & 0.35 \\
\hline GAMA & -6.95 & -3.13 & -0.48 & 0.10 & 7.24 & 0.00 \\
\hline WOLASP & 3.00 & 25.50 & 42.00 & 82.50 & 510.00 & 13.50 \\
\hline
\end{tabular}

Tabela 12 - Momentos das variáveis da hidrógrafa - HIDVAR

\begin{tabular}{|c|c|c|c|c|c|}
\hline HIIDVAR & $\mathbf{n}$ & $\mathrm{Xm}$ & $\mathbf{S}$ & $\mathbf{M B}$ & $\mathrm{CV}$ \\
\hline BPSTON & 81 & 62.47 & 52.29 & 1.93 & $\overline{83.70}$ \\
\hline I.AY, BDA & 81 & 398.02 & 182.94 & 2.09 & 45.98 \\
\hline DELTA & 81 & 1.71 & 1.41 & 1.24 & 82.71 \\
\hline GAYA & 81 & -1.30 & 2.49 & 0.13 & -190.68 \\
\hline MoLSP & 71 & 74.51 & 88.33 & 2.69 & 118.55 \\
\hline
\end{tabular}

$\sigma$

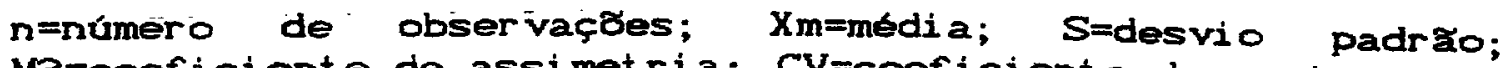
M3=coeficiente de assimetria; CV=coeficiente de variação. 


\section{3. A model agem}

Nesta etapa serão apresentadas e analisadas as equações de regressão obtidas após os passos realizados pelo PASH em 5.2. sendo possivel então a modelagem do sistema.

Os Modelos de simulação de hidrógrafas com base no Sistema Johnson de Distribuição apresentaram as seguintes equaçóes de regressão :

$$
\begin{array}{r}
\hat{\gamma}=-0.08(\text { volcho/p75cho }-2.83(\operatorname{Ln}(p 25 \mathrm{ch} 3))+ \\
1.79(\mathrm{p} 25 \mathrm{ch} 1)-2.11(\operatorname{Ln}(\mathrm{p} 50 \mathrm{ch} 1)) \\
\mathrm{R}^{2}=47.2 \% \quad F(4,77)=17,3^{* * *}
\end{array}
$$

$$
\begin{array}{r}
\hat{\delta}=[100.89(p 100 c h 0)-424.79(p 50 c h 0)+ \\
+293.97(p 75 c h 0)]] \hat{\lambda} \\
R^{2}=67.2 \% \quad F(4,78)=545^{* *}
\end{array}
$$

$$
\begin{aligned}
& \hat{\lambda}=326.0 \operatorname{Ln}(\mathrm{p} 100 \mathrm{cho})+ 12.7(\mathrm{volcho} / \mathrm{p} 100 \mathrm{cho}) \\
&+157 \operatorname{Ln}(\mathrm{p} 25 \mathrm{ch})-2.6(\mathrm{vol} \operatorname{cho} / \mathrm{p} 25 \mathrm{cho}) \\
& \mathrm{R}^{2}=93 \% \quad \mathrm{~F}(4,77)=258,5
\end{aligned}
$$


$\hat{\varepsilon}=\exp [5.65 \mathrm{Ln}(\mathrm{p} 25 \mathrm{cho})+0.08(\mathrm{volch} / \mathrm{p} 100 \mathrm{cho})-$

- 0.57 p50chos + 0.02(volcho/peschos ] $R^{2}=91.1 \% \quad F(4,77)=16,8^{* * *}$

$$
\begin{aligned}
& \hat{V}=\exp \left[0 . 8 4 \left(\operatorname{volchos}^{1 / 2}+0.55(\operatorname{Ln}(\text { volch } 12)-\right.\right. \\
& 0.08(\operatorname{Ln}(t) h 01) * \operatorname{Ln}(\text { valch1) }] \\
& \mathrm{R}^{2}=97.6 \% \quad \mathrm{~F}(3,79)=942,1
\end{aligned}
$$

onde, $\hat{v} \in$ a estimativa do volume da hidrografa.

Os parametros $\hat{\lambda}$ e $\hat{\varepsilon}$ obtiveram correlação somente com variáveis $($ percentis e intensidades de chuva) relacionadas com a chuva zero (chos, confirmando a hipótese básica de que, pelo menos com relação ao infcio e fim da hidrografa, a cho tem efeito marcante na geração desses dois parámetros. Essas variáveis são pescho p5ocho p100cho e volcho/p100cho.

o parámetro $\hat{\gamma}$ no entanto só se relaciona com a intensidade da chuva zero (atual) ao percentil 75 , isto é com a variável (volcho/p75chos. Esta tem o menor efeito na 
composição de $\hat{\gamma}$. Alem desta variável as chuvas ch3 e ch1 tambom atraves de percentis, mantém forte influencia em $\hat{\gamma}$. Este parámetro do Sistema de Distribuição Johnson tem efeito na escala da distribuiça formada, podendo comprometer a simulação das hidrógrafas, o que desde já pode-se prever pela grande diferença do $R^{2}(47.2 \%$ em relação ao $R^{2}$ dos outros parâmetros.

O parametro $\hat{\delta}$, por outro lado, tem relação com os percentis p100cho e p75cho,sendo que este úl timo tem grande efeito na formação de $\hat{\delta}$. Provalvelmente estas relaçes com os percentis deve-se a inclusão de $\hat{\lambda}$ na equação de $\hat{\delta}$.

A estimativa do volume da hidrografa $\hat{v}$ mantém rel ação não só com a cho, mas tambem com a ch1. Cabe observar a inclusão da variável volcho com expoente $1 / 2$, e variáveis combinadas.

5. 3. 2. MSHA

Os modelos de simulação de alturas das hidrógrafas, geraram 35 equaçóes correlacionando as alturas de vazão $\mathrm{Hi}$ (em intervalos de 20 minutos), com as variáveis das chuvas anteriores.

$\mathrm{Na}$ sequiéncia são apresentadas as equaçర̃es de regressão. 
LncHeOs $=0.05$ (volcho+volchs) $-0.68 \mathrm{Ln}(\mathrm{p} 50 \mathrm{chO})+$

$+70.79(p 75 c h 0 \hat{\lambda}$

$$
R^{2}=70.03 \% \quad F(3,79)=83.51^{* *}
$$

Ln(H4O) $=0.06$ ( volcho+volchs) $-0.86 \mathrm{Ln}(\mathrm{p} 50 \mathrm{chO}+$

$+66.63(\mathrm{p} 100 \mathrm{cho \hat {n }}$

$R^{2}=80.24 \% \quad F(3,79)=106.96^{* * *}$

LnCHGO) $=0.07$ (volcho+volch5) - $0.21 \mathrm{Ln}(\mathrm{p} 50 \mathrm{chO})$

$$
R^{2}=75.80 \% \quad F(3,79)=82.46^{* * *}
$$

LnCH8OS $=-0.73 \mathrm{Ln}(\mathrm{p} 75 \mathrm{chos}+0.76 \mathrm{Ln}(\mathrm{vol}$ chos

$$
\mathrm{R}^{2}=92.40 \% \quad \mathrm{~F}(3,79)=320.00^{* * *}
$$

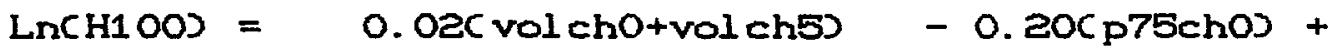

$+0.62 \ln C$ volchos

$\mathrm{R}^{2}=93.69 \% \quad F(3,79)=390.80^{* * *}$

Ln(H120) $=0.05$ (volcho+volch5) -0.17 p5ochos +

$+0.72 \ln (p 25 c h 1)+0.02($ volcho-volch 3$)+0.14 \operatorname{Ln}($ tcho 4$)$

$$
R^{2}=88.73 \quad F\left(5,773=121.28^{* * x}\right.
$$

$\operatorname{Ln}(\mathrm{H} 140)=-0.20\left(\right.$ p5Ochos $+0.39(\text { volchos })^{0.5}+0.07(t$ cho 4$)$

$$
R^{2}=84.74 \quad F(6.75)=225.09^{* *}
$$

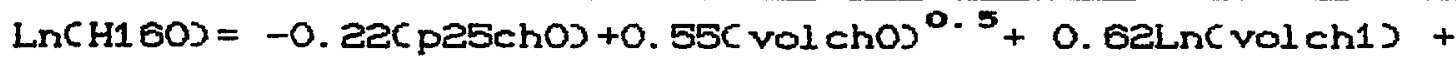

-0.16 (LnCtcho1) $* \operatorname{Ln}(\operatorname{vol} c h 13)-0.14$ (p25che) $+0.01(t c h 01)$

$$
R^{2}=95.36 \quad F(7.74)=217.37^{* * x}
$$




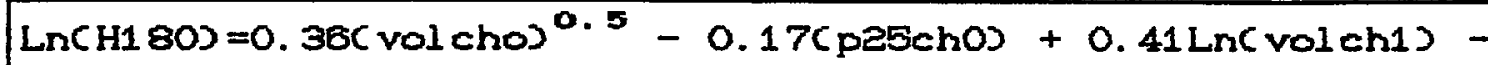
$+0.10(\operatorname{Ln}(t c h 01) * \operatorname{Ln}($ vol ch1 $)$

$$
R^{2}=93.53 \quad F(5,76)=219.60^{* *}
$$

$\operatorname{Ln}(H 200)=0.04($ vol chos $-54.6(p 75 c h 0 / \lambda)+0.54 \operatorname{Ln}($ vol ch1 $)-$ $+0.14(\operatorname{Ln}($ tcho1 $) * \operatorname{Ln}($ vol ch1 $) 3+1.24 \operatorname{Ln}(p 75 c h 0)-0.27 \beta s(p 75 c h 0)$

$$
R^{2}=94.13 \% \quad F(8.72)=144.44 *
$$

$$
\begin{array}{r}
\text { Lnche2O }=0.05(\text { volchos }+1.01(\mathrm{p} 100 \mathrm{ch} 1)-0.18(\mathrm{p} 1 \text { ooch } 1) \\
\mathrm{R}^{2}=91.02 \% \quad \mathrm{~F}(5,73)=147.94^{* *}
\end{array}
$$

Ln(H24O) $=0.04$ (volchos +0.7OLn(volch1) -

$+0.2(\operatorname{Ln}(t c h 01) * \operatorname{Ln}($ volch1) $+0.4 \operatorname{Ln}(p 100 c h 0)$

$$
R^{2}=91.29 \quad F(6,70)=122.30^{* * *}
$$

Lnch260) $=0.001\left(\right.$ volchos ${ }^{2}-228.7($ paschon $)+$

$0.24 \operatorname{Ln}($ volch 1$)+1.28 \mathrm{Ln}(\mathrm{p} 25 \mathrm{chos}$

$$
\left.R^{2}=86.73 \% \quad F(4,68)=111.07\right)^{* * *}
$$

Lnchesos $=0.001\left(\right.$ volchos ${ }^{2}-279.5($ pescho $\hat{\lambda})+$

$1.48 \mathrm{Ln}(\mathrm{p} 25 \mathrm{chos}+0.24 \mathrm{Ln}(\mathrm{vol} \mathrm{ch} 1)$ $R^{2}=90.64 \% \quad F(4.59)=142.88^{* *}$

$\operatorname{Ln}(\mathrm{H} 300)=0.0155(\mathrm{volchO})^{2}$

$$
R^{2}=75.28 \% \quad F(1,59)=179.63^{* *}
$$




$$
\begin{aligned}
\operatorname{Ln}(\mathrm{H} 320)= & 0.00159\left(\mathrm{vol}_{\mathrm{chOS}}{ }^{2}\right. \\
\mathrm{R}^{2} & =77.58 \% \quad F(1.56)=193.53^{* *}
\end{aligned}
$$

LnCH34OS $=0.00137\left(\right.$ volchos ${ }^{2}+0.00482($ tcho2 $)$

$$
R^{2}=81.87 \% \quad F(2,51)=115.14^{* *}
$$

LnCH360) $=0.0008\left(\right.$ volchos $^{2}+0.27($ p50chos

$$
R^{2}=85.17 \% \quad F(2,46)=132.09^{* *}
$$

LnCH380) $=0.0008$ (volchos ${ }^{2}+0.23($ p50cho

$$
R^{2}=89.43 \% \quad F(4,41)=88.69^{* * *}
$$

$\operatorname{Ln}(\mathrm{H} 400)=0.0007(\mathrm{volchos})^{2}+0.10$ p100chos

$$
R^{2}=88.54 \% \quad F(4,40)=77.22^{* *}
$$

LnCH4ZO) $=0.0013\left(\right.$ vol chos ${ }^{2}$

$$
R^{2}=76.26 \% \quad F(1,38)=122.07^{* * *}
$$

LncH440) $=0.0008$ volchos $^{2}+0.11(\mathrm{p} 100 \mathrm{cho})$

$$
R^{2}=01.13 \% \quad F(2,31)=150.20^{* *}
$$

Ln(H46O) $=0.00044\left(\right.$ volchos ${ }^{2}+0.21(p 50$ cho $)$

$$
R^{2}=86.21 \% \quad F(3,30)=62.53^{* *}
$$


LnCH480) $=0.0006\left(\right.$ volchos ${ }^{2}+0.35 \operatorname{Ln}(p 100 c h 0)$

$$
R^{2}=84.70 \% \quad F(Z, 27)=74.73^{* *}
$$

LncH500) $=0.0005\left(\right.$ volchos ${ }^{2}+0.22(p 50 c h 0)$

$$
R^{2}=90.52 \% \quad F(2,23)=109.87^{* *}
$$

LnCH5ZOS $=0.001$ volchos $^{2}$

$$
R^{2}=80.20 \% \quad F(1.23)=93.19^{* *}
$$

$\operatorname{Ln}(\mathrm{H} 540)=0.001\left(\mathrm{vol}_{\mathrm{ChO}}{ }^{2}\right.$

$$
R^{2}=86.00 \% F(1,18)=110.61^{* *}
$$

$\operatorname{LnCH580S}=0.0005(\mathrm{vol} \mathrm{chos})^{2}$

$$
R^{2}=79.03 \% \quad F(2,14)=26.39^{* *}
$$

LncH580) $=0.296$ p50chOS

$$
R^{2}=81.34 \% \quad F(1,12)=52.32^{* *}
$$

LnCH6OO) $=0.26($ p5Ochos

$$
R^{2}=75.71 \% \quad F(1.10)=31.18^{* *}
$$

$\operatorname{Ln}(H 620)=1.24 \mathrm{Ln}(\mathrm{p} 50 \mathrm{chO})-193(\mathrm{p} 1$ 00cho $\hat{\lambda})$

$$
R^{2}=73.31 \% \quad F(3,6)=5.49^{* *}
$$

LnCHE4OS $=0.73 \mathrm{Lr}(\mathrm{p}$ (5OChO

$$
R^{2}=95.10 \% \quad F(1,4)=78.00^{* *}
$$


5. 4. A Simulução ( Vali dação dos modelos)

A etapa posterior a obtenção das equaçós de regressão para cada modelo sugerido CMSHJ e MSHAJ o a simulação e validação das hidrógrafas.

Para o processo de simulação de hidrografas foi utilizado como entrada dos modelos as 82 chuvas atuais, acompanhadas das chuvas anteriores. Deste modo foi possivel aferir (validar) a desempenho dos modelos, já que se dispõe das hidrógrafas observadas, que representam as respostas do sistema aos estimulos ou entradas das chuvas atuais anteriores.

Deve-se lembrar que o estudo de modelagem e simulação das hidrografas tem grande importancia em previsáo de eventos hidrologicos, principalmente aqueles ligados ao estudo de cheias (enchentes), bem com o entendimento da dinámica da água numa bacia hidrografica e a geração do deflúvio.

Para tanto, há necessidade de validação do modelo proposto, estudos de condiçర̃es de excessão e se possivel, determinação da sua faixa de atuação.

- estudo de simulação foi feito levando-se em conta a distribuicão de frequéncia dos volumes gerados pelas hidrografas (Figura 24). Dentro de cada classe volumetrica sugerido uma classificação das simulaçôs existentes.

Para tal, fol necessário iniclalmente "medir" - ajustamento feito pelos modelos CMSHJ e MSHA , tendo-se o objetivo de comparar os diversos ajustes.

A primeira ideta foi utilizar o teste de Kolmogorov-Smirnov para efetuar essas comparaçбes múl tiplas com o intuito de classificar os ajustes. Este teste que tem como fator discriminante a maior distancia ertre as distribuiçôes acumul adas observadas e esperadas, não tendo sensibilidade suficiente para por exemplo, captar 
diferenças do tempo de ascensão da hidrografa.

Como visto no capitulo 2 , as hidrografas possuem parámetros de caracterização como tempo de ascensão e recessão, inicio e fim do escoamento, vazão no ponto máximo. O ideal seria a utilização de uma média ponderada dos desvios, que levasse em conta os parametros acima.

E sujerido um critério de classificaça em que se utiliza um discriminante de ajustes (Ra), baseado numa relação entre a media e o desvio padrão dos desvios absolutos. A primeira vista poderia se pensar no coeficiente de variação CCV, mas o critério tem com ponto básico os seguintes principios CFigura 29):

1. Calcula-se a diferença absoluta para cada uma das 82 simulaçốes, obtendo-se assim a média e o desvio padrão dos desvios absolutos.

2. Num sistema de eixos cartesianos, plota-se no eixo das abscissas, os valores das medias e-no eixo das ordenadas, os respectivos valores dos desvios padrão.

3. Determina-se finalmente a distancia cartesiana (Ra) de cada ponto no plano, representando cada simulação, ao centro do sistema de eixos corigem, que na verdade $b$ que se deseja : medias próximos de zero com desvio padrão reduzido.

4. Dentro de cada classe de volume de hidrografa, determina-se as distancias Ra ordenado-as.

5. Deste modo pode-se determinar os percentis $33.33 \%$, $66,66 \%$ e $100 \%$ das distribuiçoses de Ra nas classes de volume, obtendo-se então tres classes de simulação : classe I, II e III (boa, media e ruim, por exemplo).

Observa-se que o coeficiente de variação na verdade está representado pela inclinação de uma dada reta. Deste modo pode-se ter várias distribuiç̧es com o mesmo CV, mas com conportamentos diferentes com relação aos desvios 
do ajuste das distribuiçర̋es. A distáncia Ra torna-se, entăo mais indicada para "medir" a eficiencia de um dado ajustamento.

5

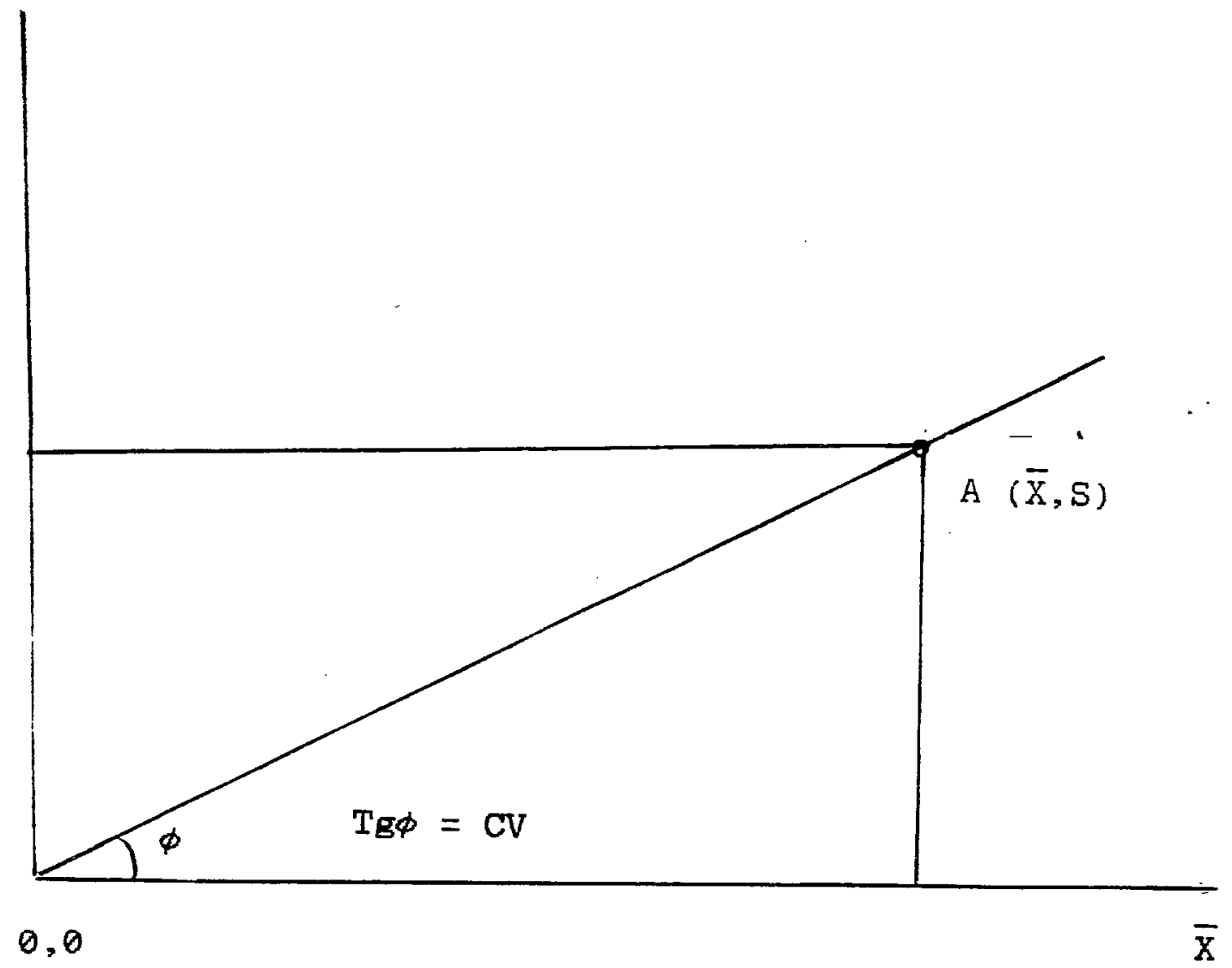

Figura 29 . - Metodo de classificaça de hidrografas': distancia "Ra". $S=$ desvio padrão; $x=$ madia; $C V=$ coeficiente de $\operatorname{var} i$ ação; $\operatorname{Tg} \phi=s / \bar{x}$. 
No APENDICE 3 são fornecidos os valores de "Ra" e seus componentes (media e desvio padrãos para cada uma das 82 simulaçбos dentro de cada método existente.

No Tabela 13 esta representado a distribuição de frequiencia dos volumes das hidrógrafas.

Tabela 13. - Distribuicão de Frequéncia do volumes das hidrografas utilizadas para a model agem.

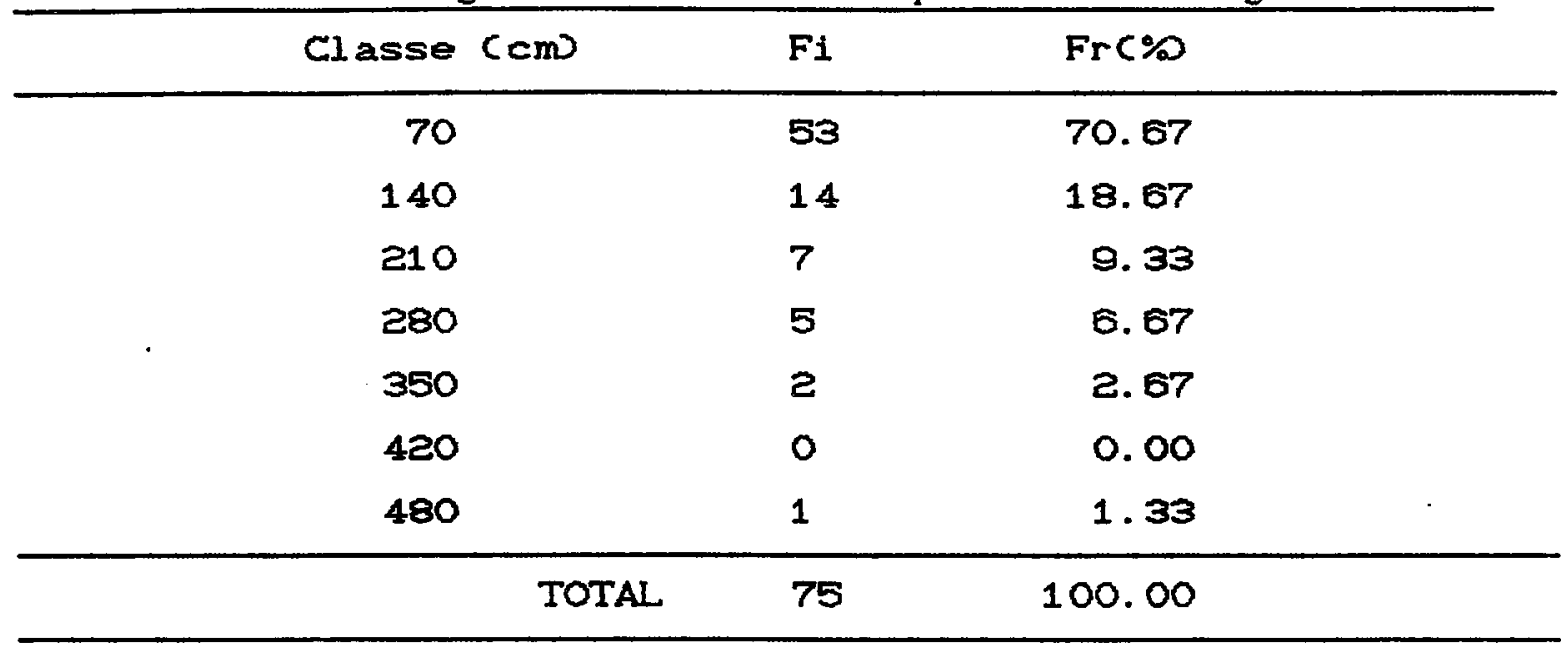

Observa-se que existe uma tendencia de crescimento dos valores de "Ra" quando a classe de volume das vazões aumenta, CAPENDICE 33.

No seguimento são apresentados os graficos das simulaçós por classe de volume de hidrógrafa.

Os modelos MSHI não obtiveram boas respostas aos estimulos das chuvas anteriores como fica verificado nos valores de "Ra" (APENDICE 3), com raras excesş̃es Figuras 30, 32 e principalmente a 41: De um total de 75 observaçð̃es de simul ação aproxi madamente 35\% apresentou uma resposta com pico de vazão no final, $10 \%$ sem respostas, $10 \%$ com pico exagerados no infcio, sendo que o restante obteve resposta mas com desvios muitos elevados.

Este fato já foi previsto anteriormente 
quando da analise dos modelos MSHJ, onde os parametros $\delta$ $\gamma$ são os principais suspeitos pela falta de sensibilidade do Sistema Johnson de Distribuição à variaçбes de condiçoses de entrada no sistema.

Já os MSHA obtiveram desenpenho por vezes melhor do que os modelos MSHJ, como comprovado pelos valores de "Ra" apresentados no apéndice 3 nas Figuras 30 a 50.

Observa-se que para classes de volume de hidrografa inferiores as previşes são melhores, quando comparadas com às útimas classes. 

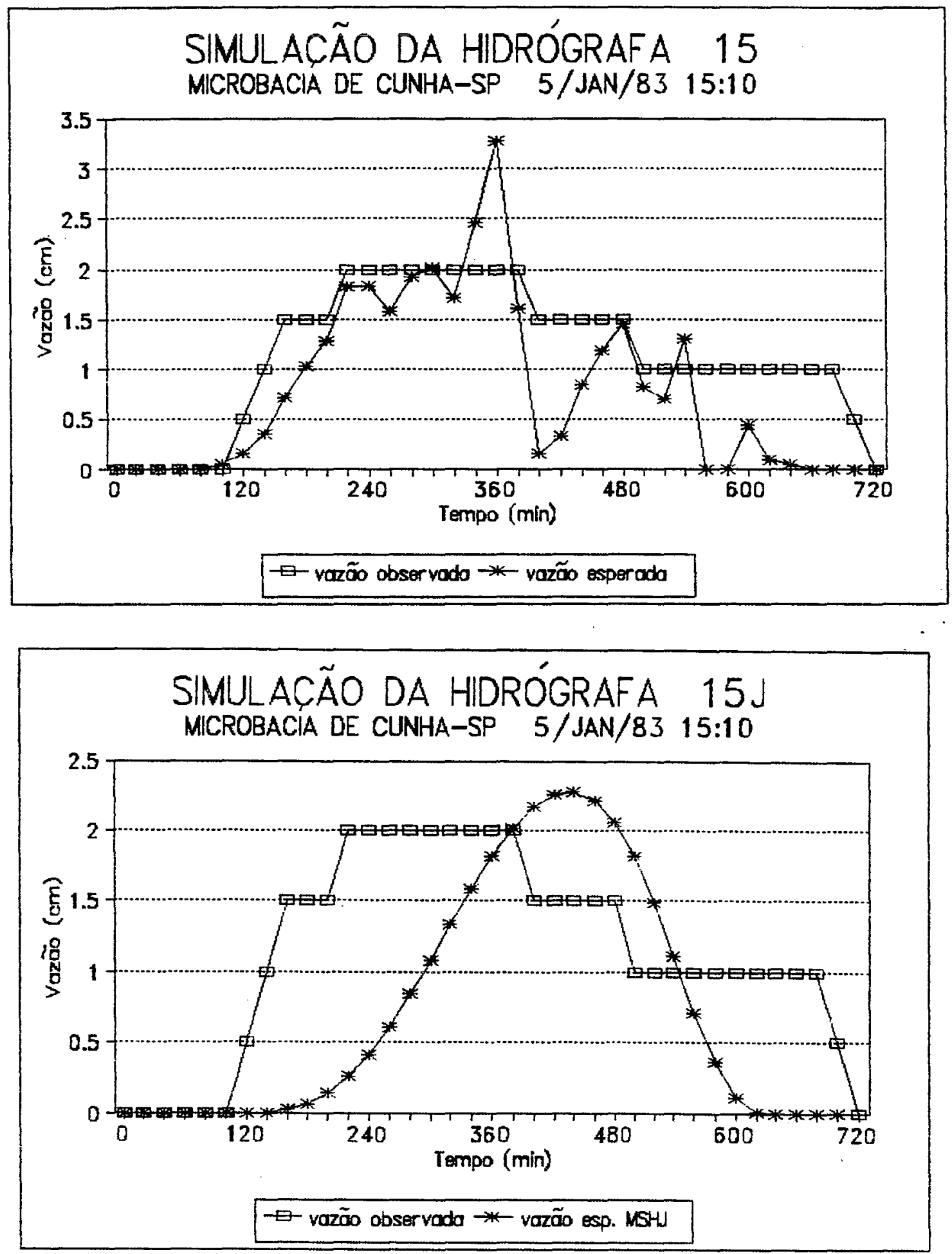

Figura 30, - Simulaç̧es de hidrografas de classe $70 \mathrm{~cm}$ de vazão, com $\mathrm{Ra}=0.62$ e $\mathrm{Ra}=0.91$ respecti vamente. 

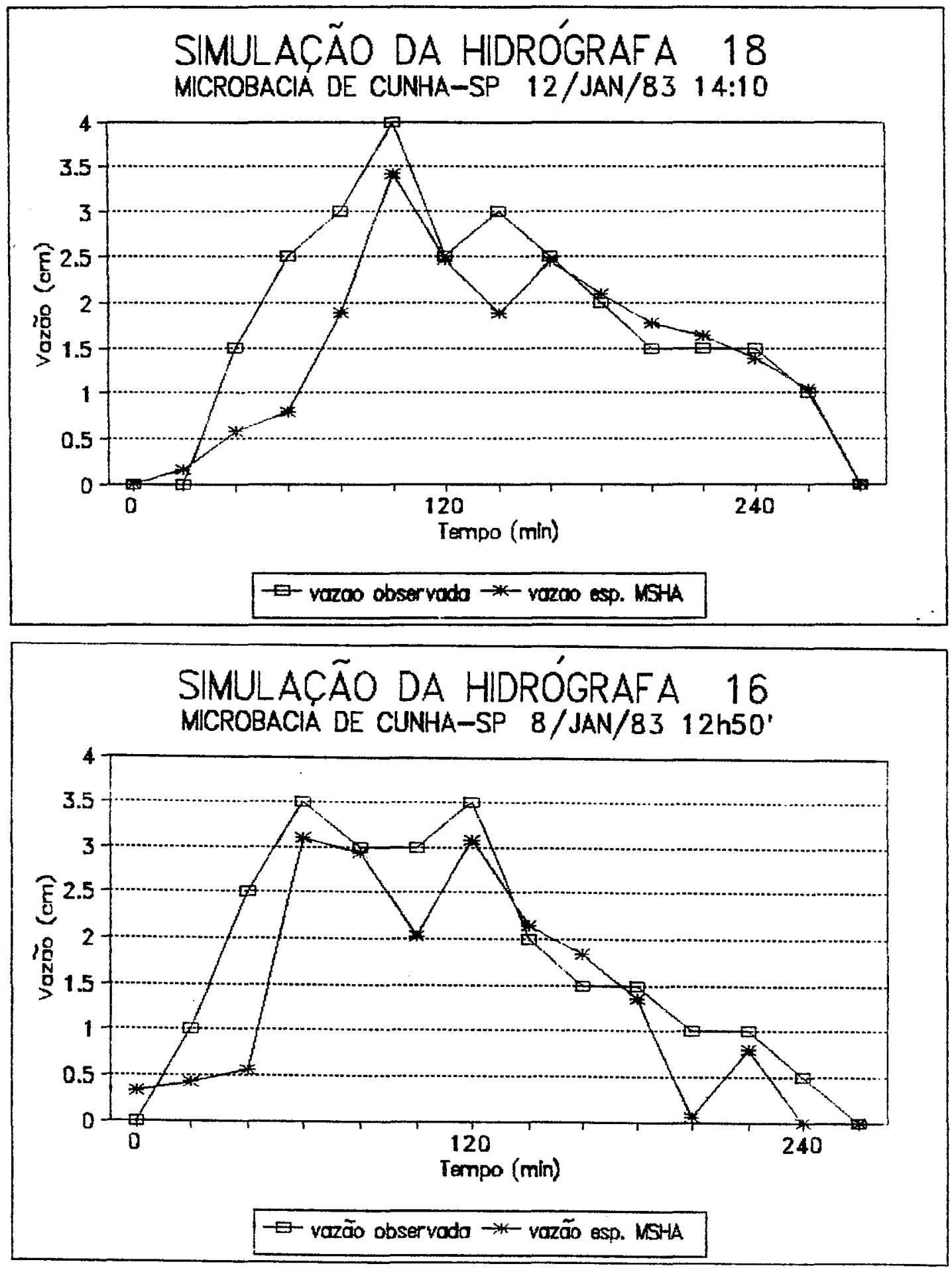

Figura 31 - Simulaçőes de hidrografas de classe $70 \mathrm{~cm}$ de vazão, com $\mathrm{Ra}=0.65$ e $\mathrm{Ra}=0.71$ respecti vamente. 

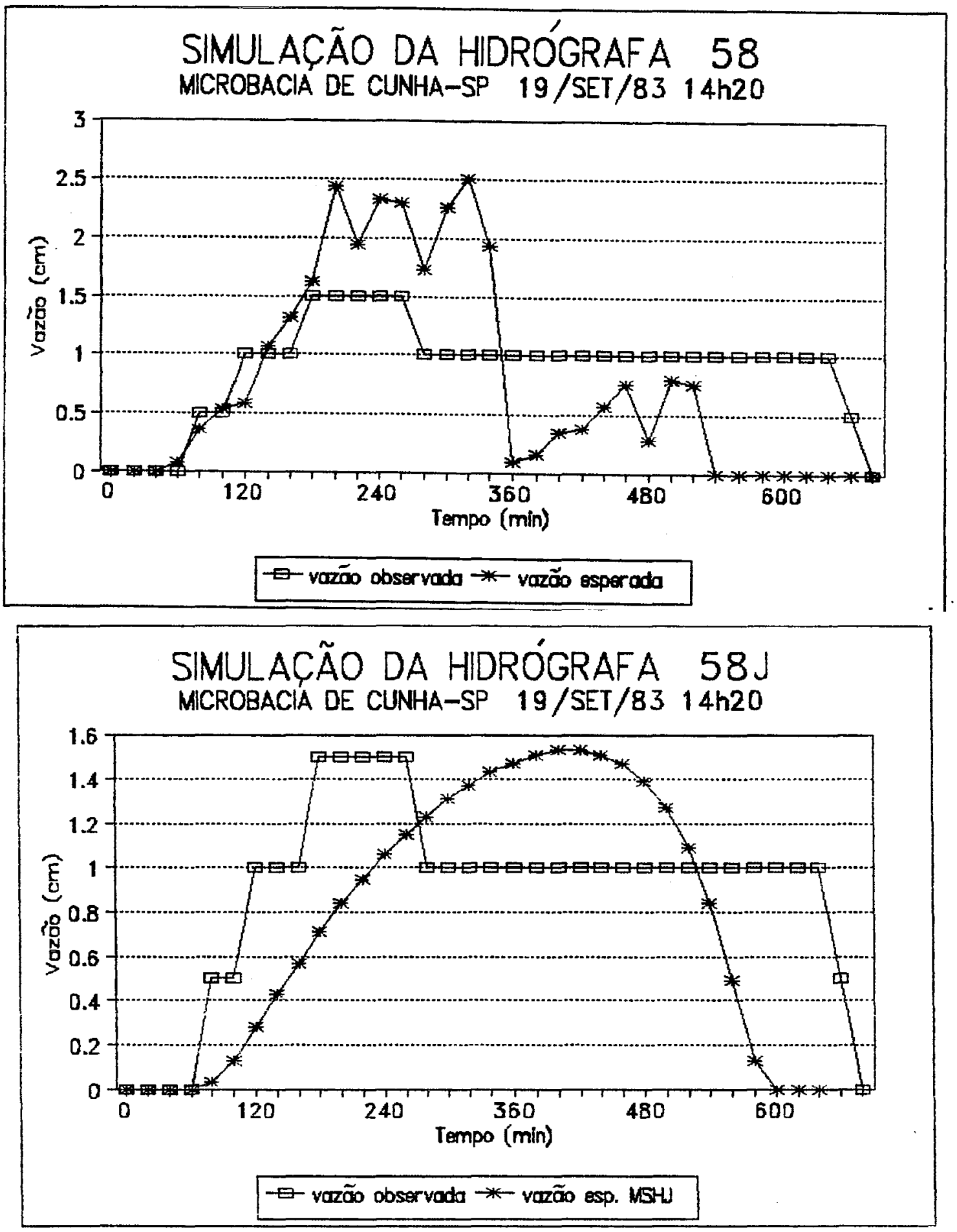

Figura 32 - Simulaçós de hidrografas de classe $70 \mathrm{~cm}$ de vazão, com $R_{a}=0.73$ e $\mathrm{Ra}=0.53$ respecti vamente. 

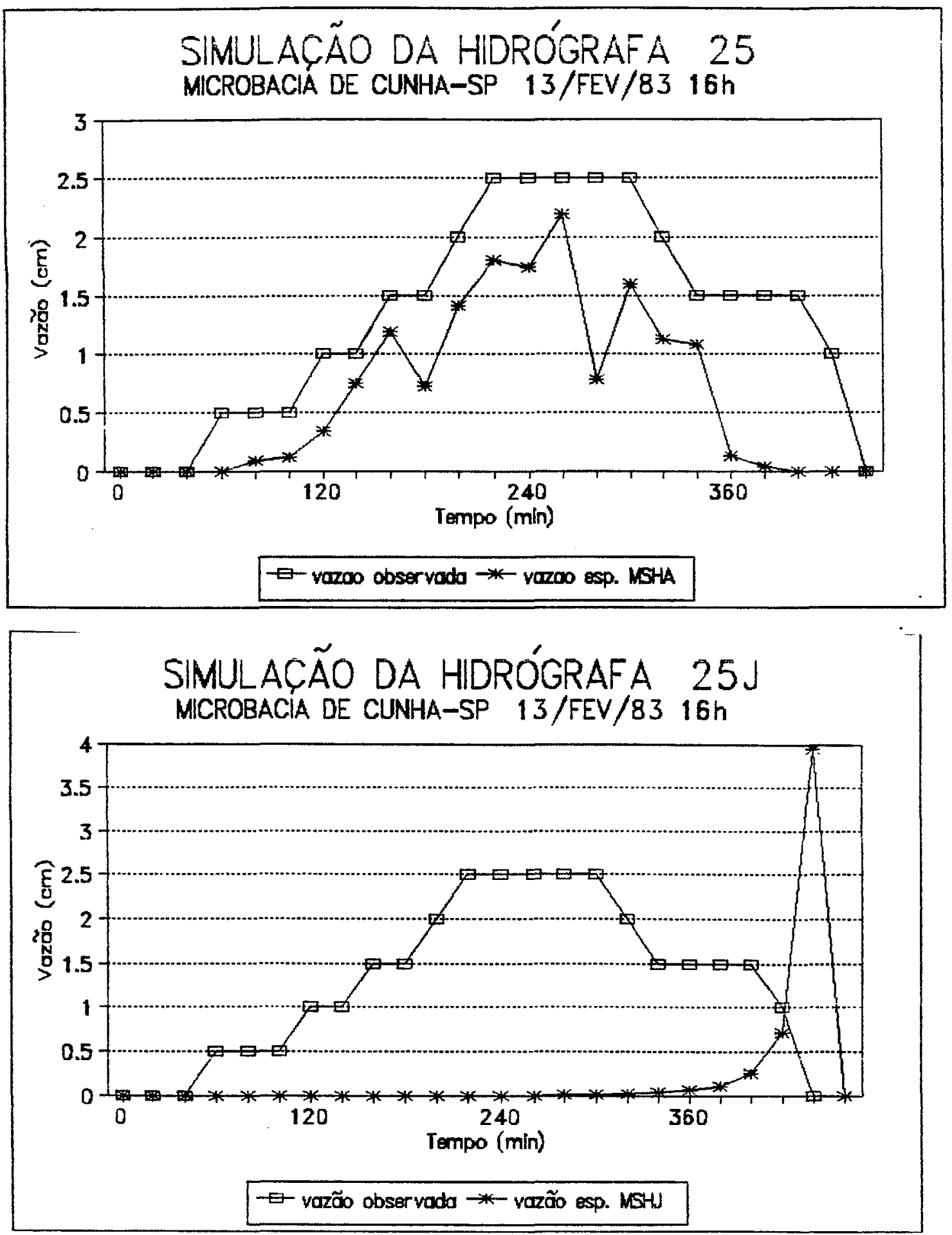

Figura 33 - Simulaçóes de hidrografas de classe $70 \mathrm{~cm}$ de 

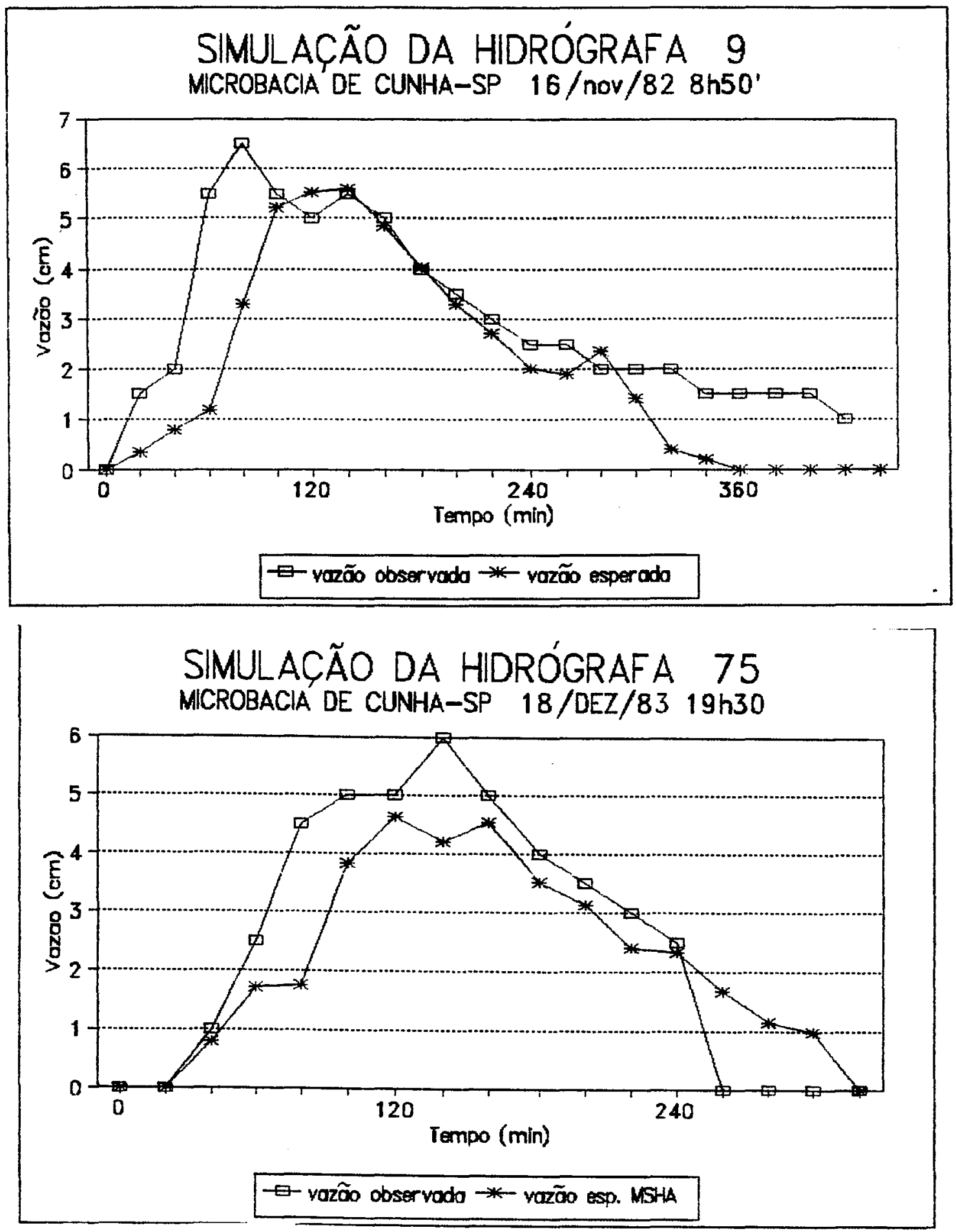

Figura 34 - Simulaçбos de hidrografas de classe $70 \mathrm{~cm}$ ue vazão, com $R a=0.95$ e $R a=1.04$ respecti vamente. 

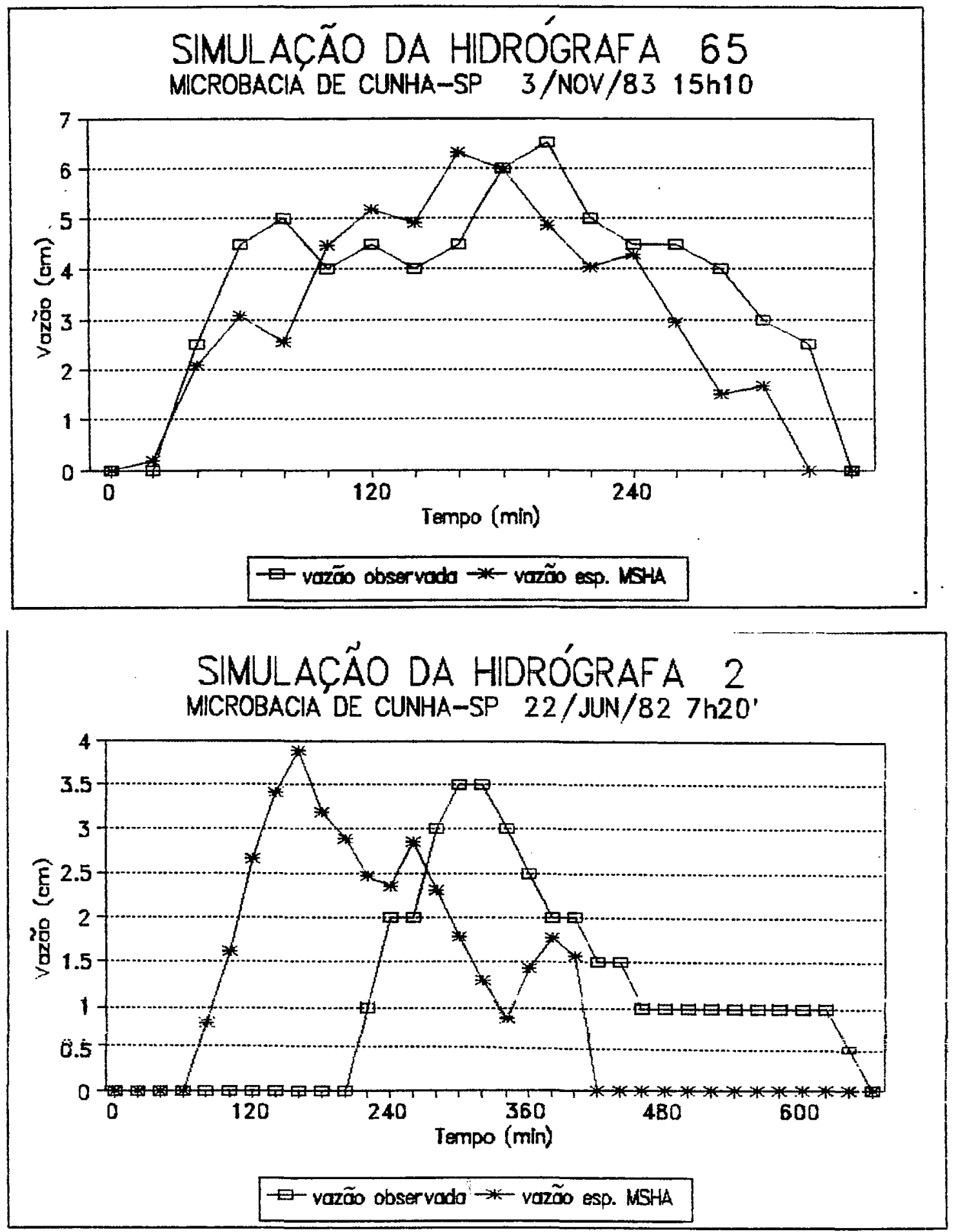

Figura 35 - Simulaçães de hidrografas de classe $70 \mathrm{~cm}$ de vazão, com $\mathrm{Ra}=1.38$ Ra= 1.81 respect 1 vamente. 

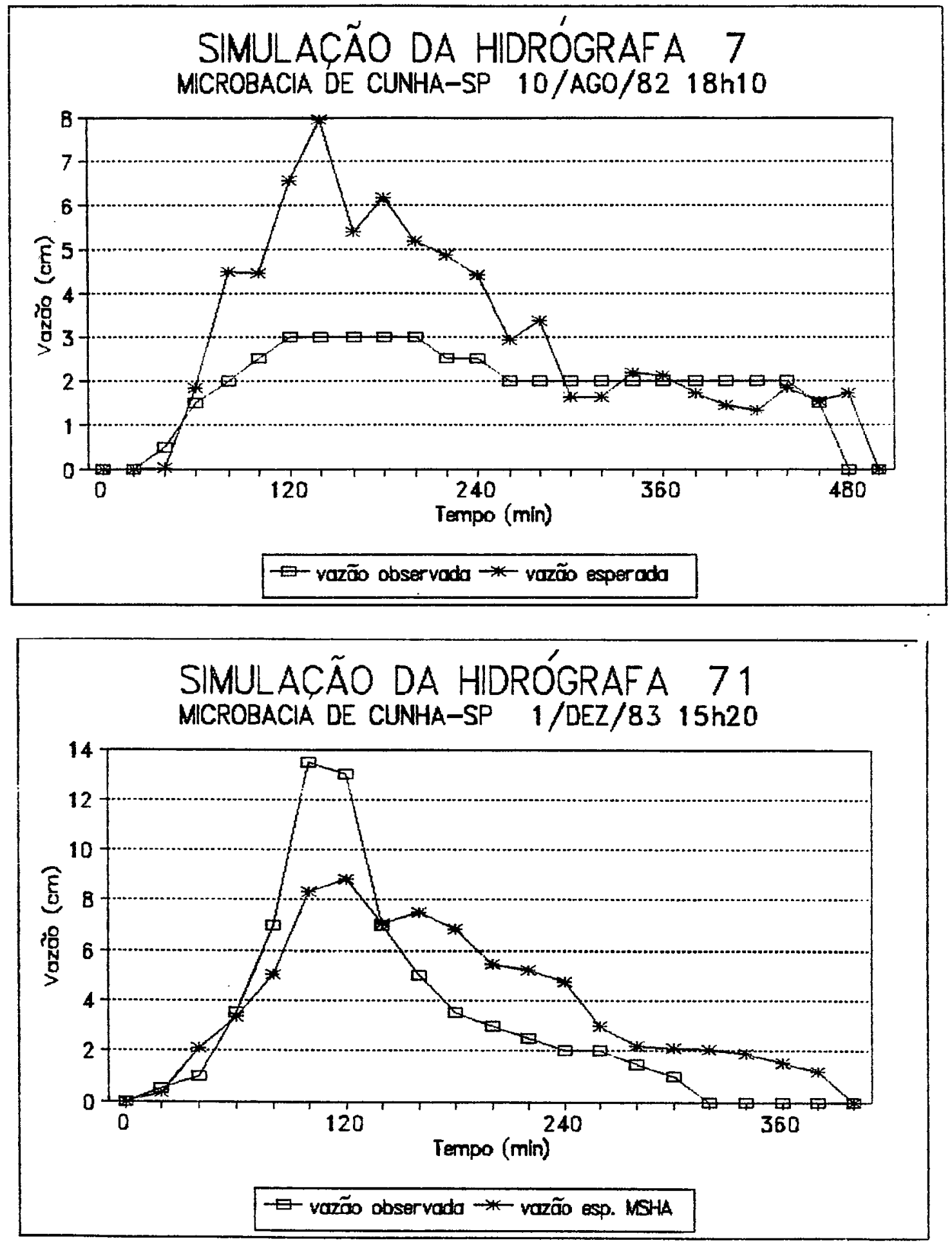

Figura 36 - Simulações de hidrografas de classe $70 \mathrm{~cm}$ de vazão, com $R a=1.72$ e $R a=1.44$ respecti vamente. 


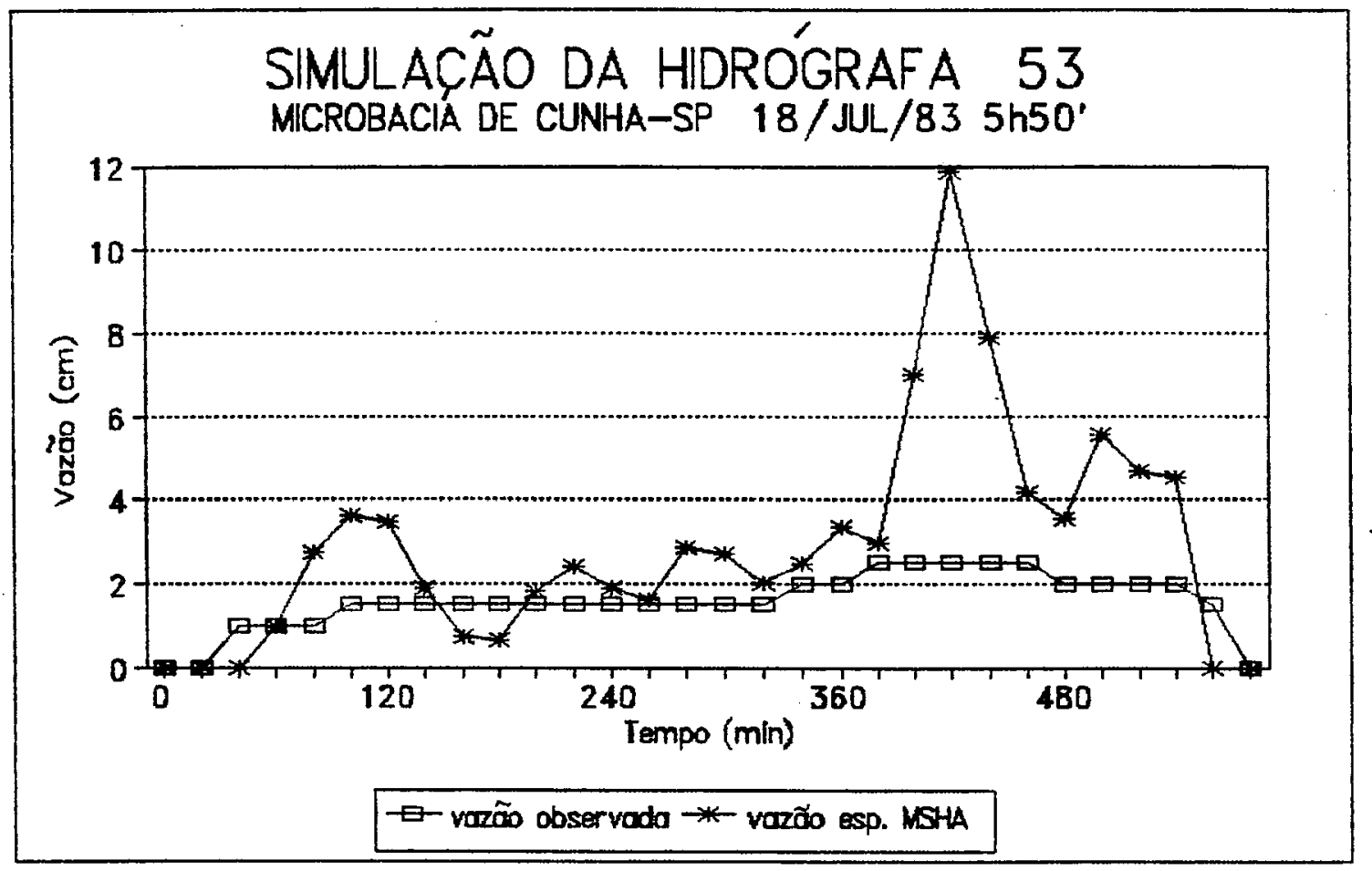

Figura 37 - Simulaçôes de hidrografas de classe $70 \mathrm{~cm}$ de vazão, com $\mathrm{Ra}=2.47$. 

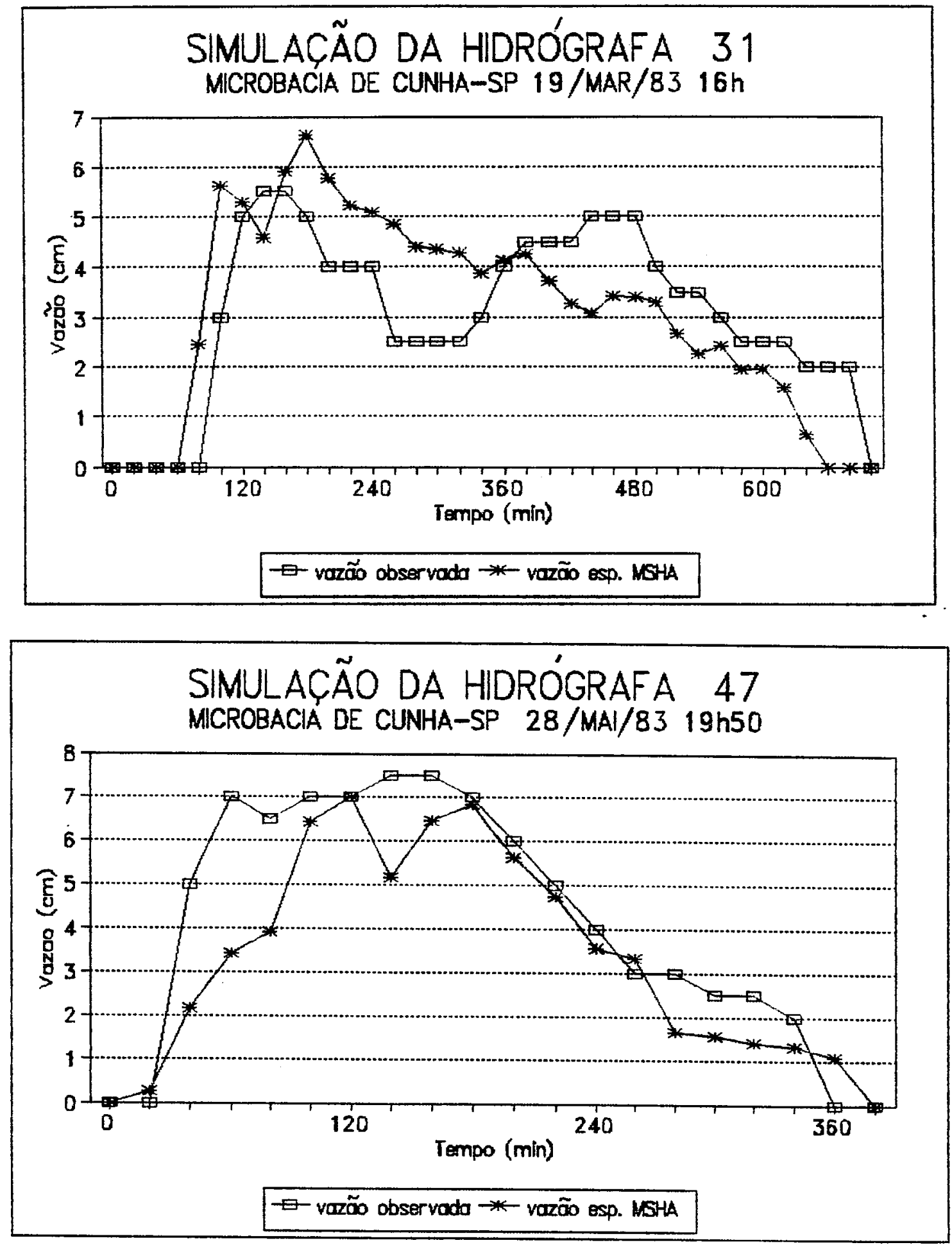

Figura 38 - Simulaçర̃es de hidrógrafas de classe $140 \mathrm{~cm}$ de vazão, com $\mathrm{Ra}=1.34 \odot \mathrm{Ra}=1.44$ respecti vamente. 

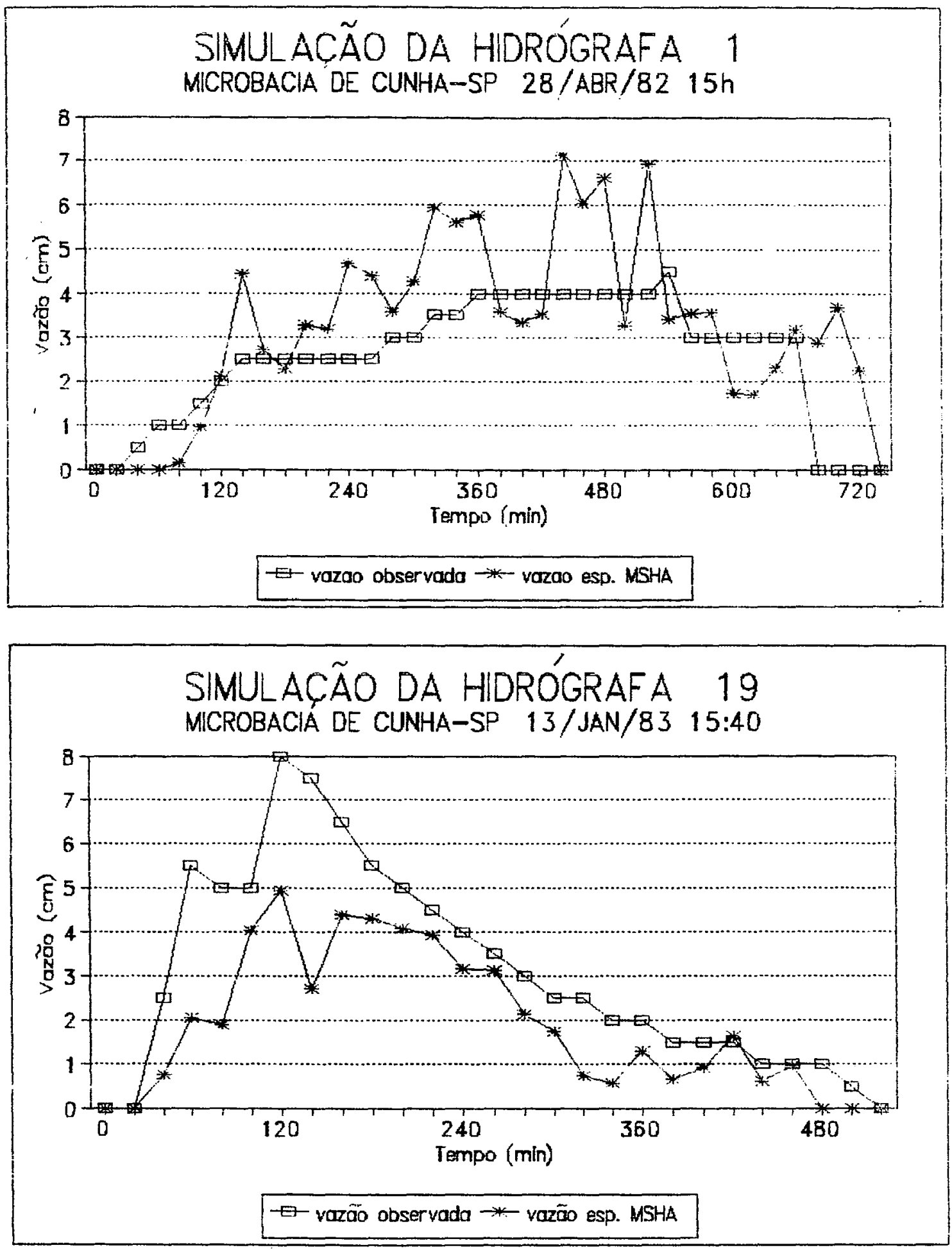

Figura 39 - Simulaçбes de hidrógrafas de classe $140 \mathrm{~cm}$ de vazão, com $\mathrm{Ra}=1.51$ e $\mathrm{Ra}=3.14$ respecti vamente. 


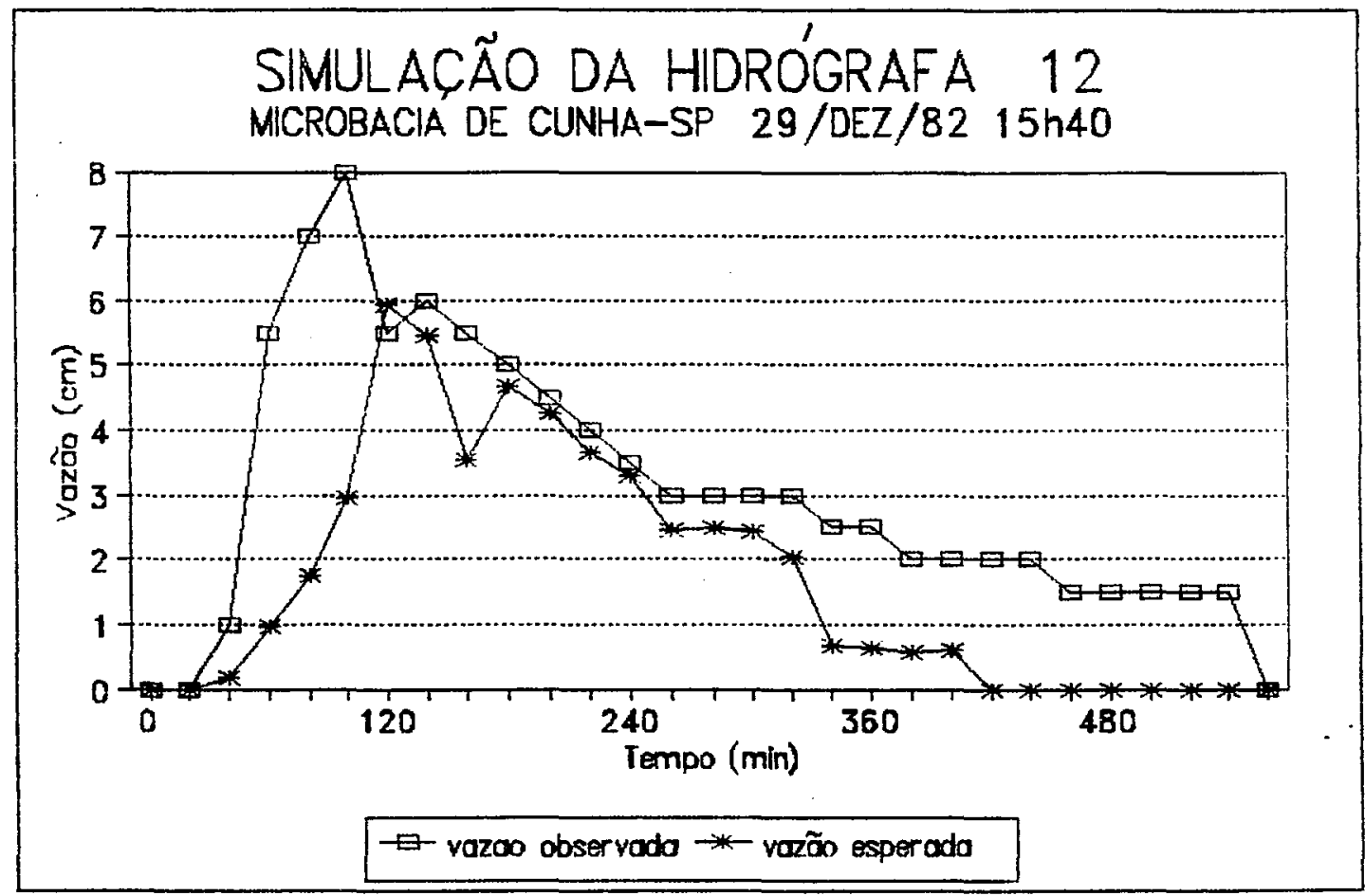

Figura 40 - Simulaçós de hidrógrafas de classe $140 \mathrm{~cm}$ de vazăo, com $R a=2.01$. 
SIMULACT̃OO DA HIDRÓGRAFA 80 MICROBACIA DE CUNHA-SP 29/OUT/82 5h

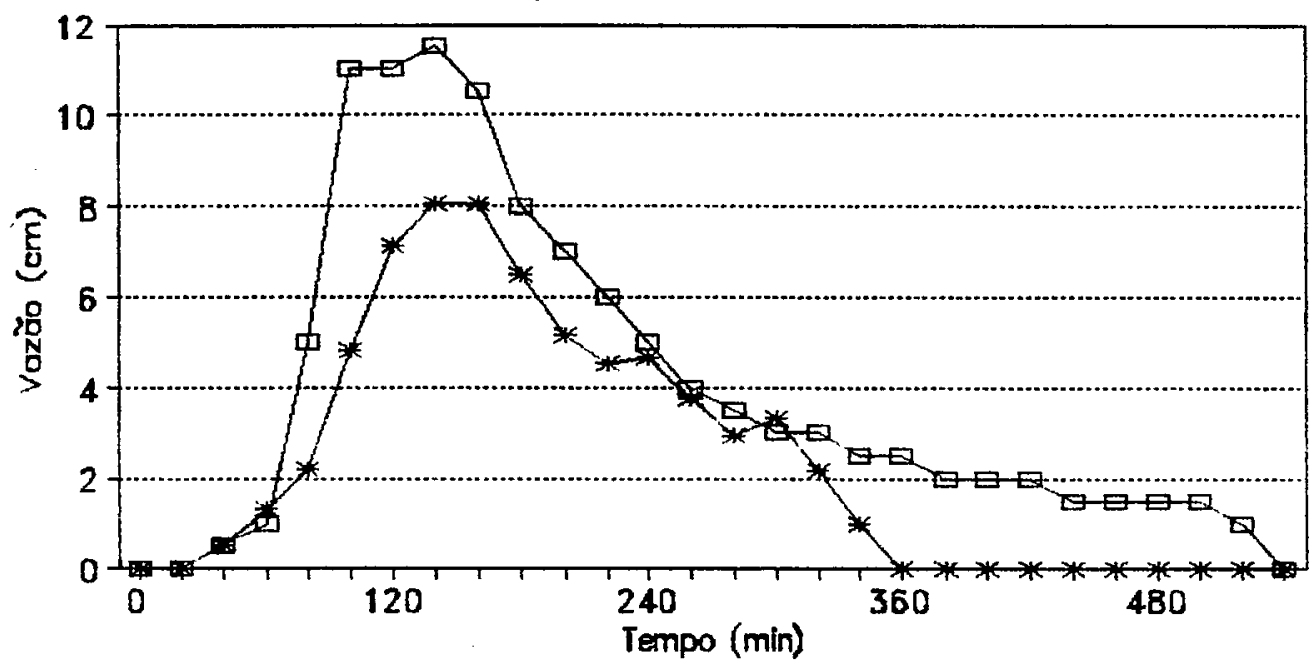

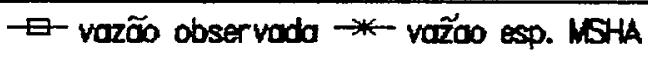

SIMULACÃO DA HIDRÓGRAFA $80 \mathrm{~J}$ MICROBACIA DE CUNHA-SP 29/OUT/82 5h

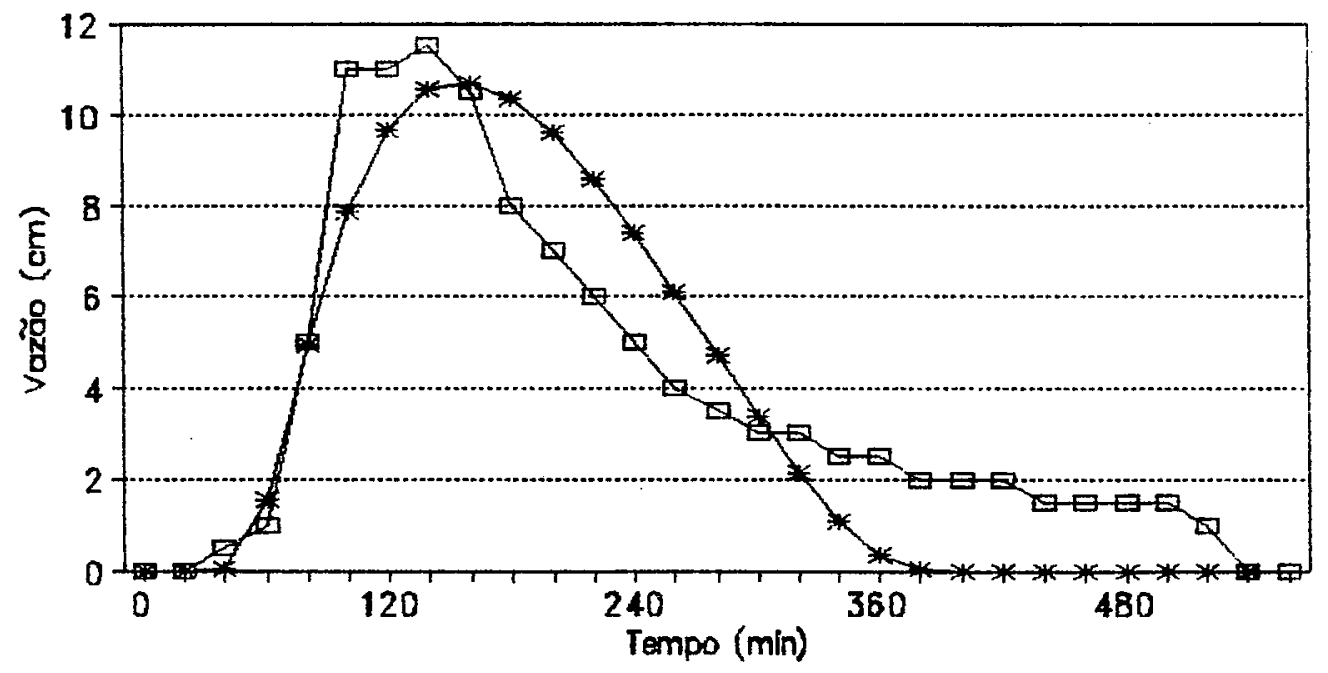

Ð yazão observada $\rightarrow$ vazão esp. MSHU

Figura 41 - Simulaçóes de hidrografas de classe $140 \mathrm{~cm}$ de vazão, com $\mathrm{Ra}=2.07$ e $\mathrm{Ra}=1.62$ respecti vamente. 


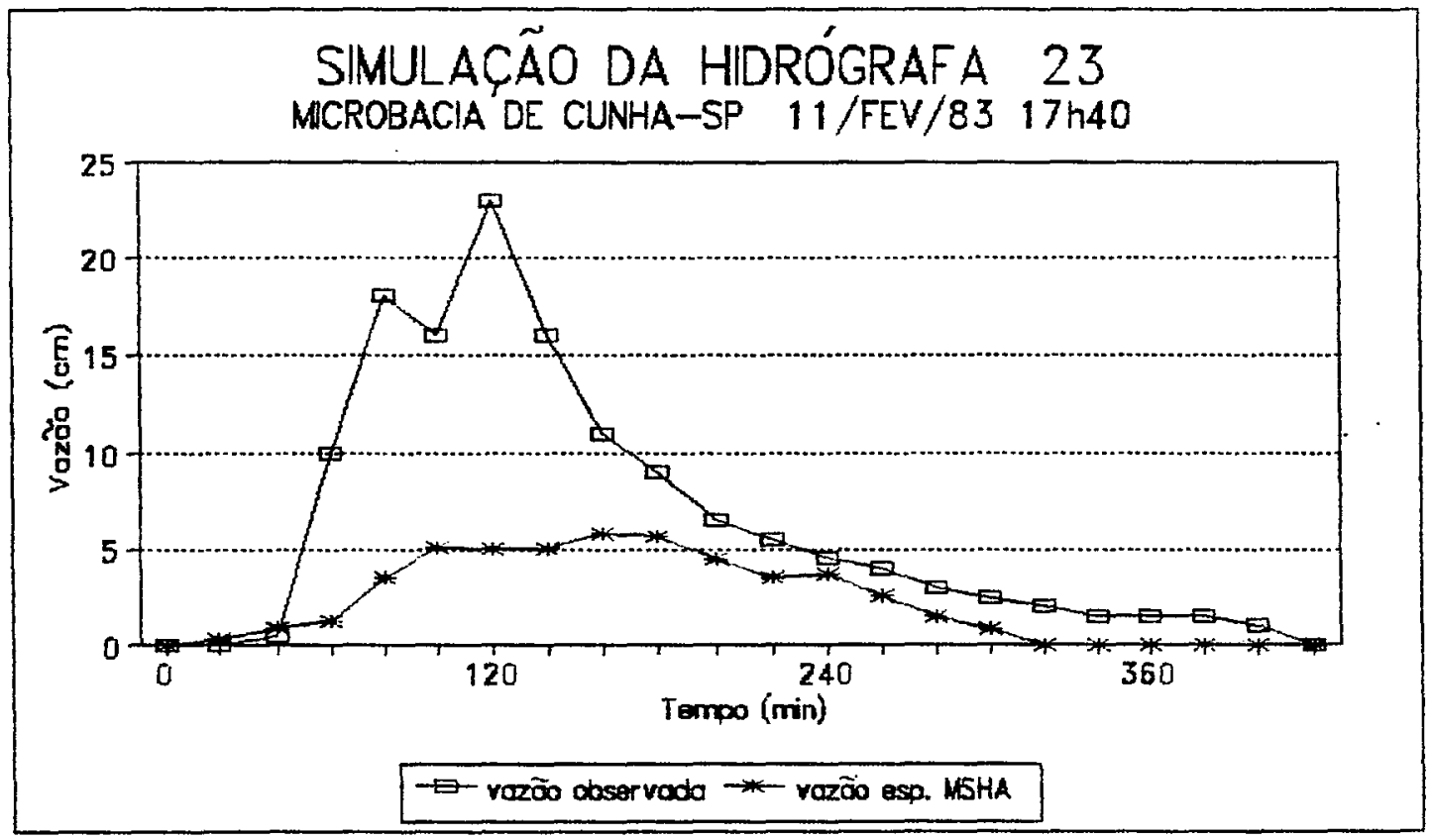

Figura 42 - Simulaçơes de hidrografas de classe $140 \mathrm{~cm}$ de vazão, com $\mathrm{Ra}=6.53$. 

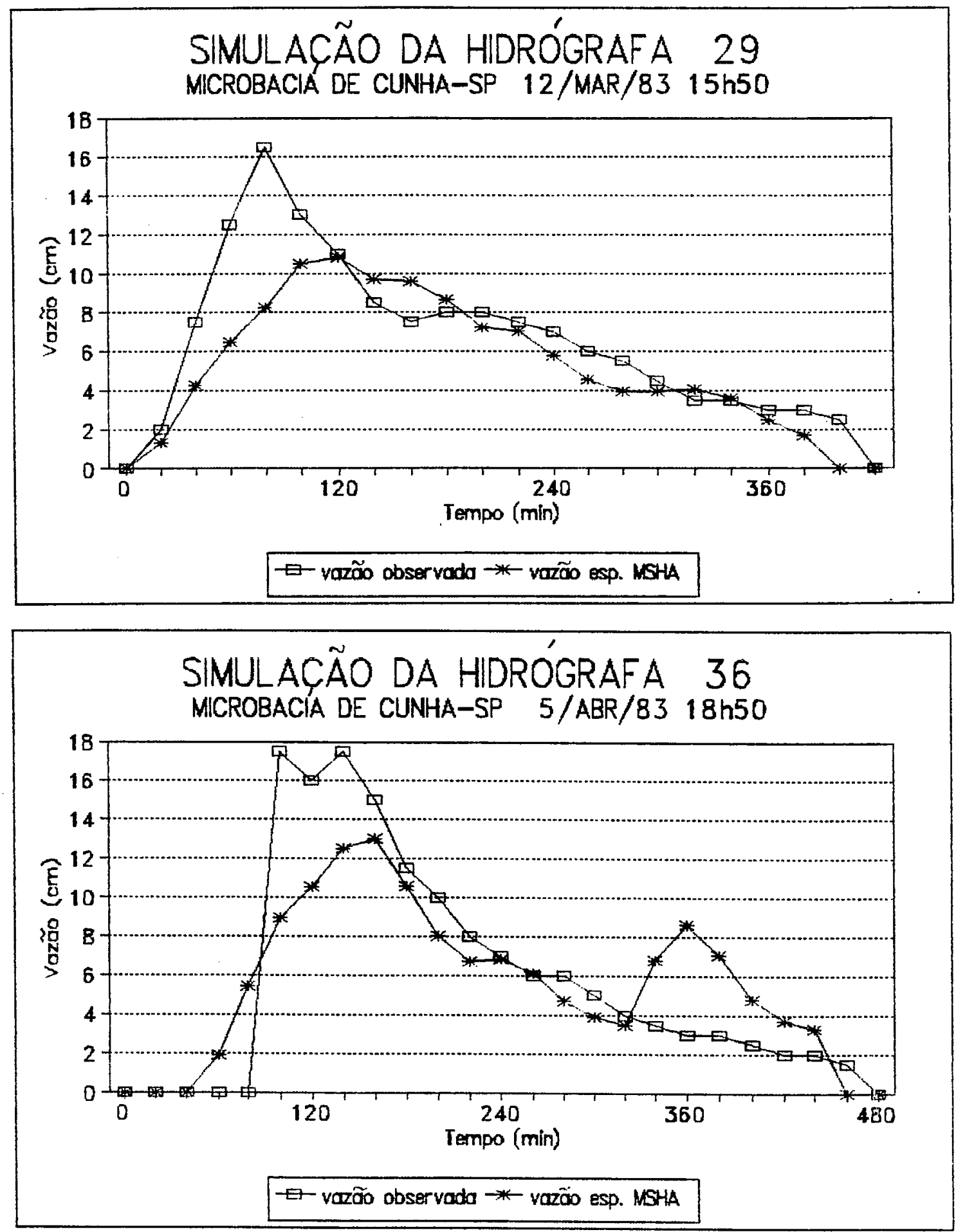

Figura 43 - Simulaçóes de hidrógrafas de classe $210 \mathrm{~cm}$ de vazão, com $\mathrm{Ra}=\mathrm{Z} .60$ e $\mathrm{Ra}=3.1 \mathrm{z}$ respecti vamente. 


\section{SIMULACÃ̃O DA HIDRÓGRAFA 59}

MICROBACIA DE CUNHA-SP 22/SET/83 13 h55

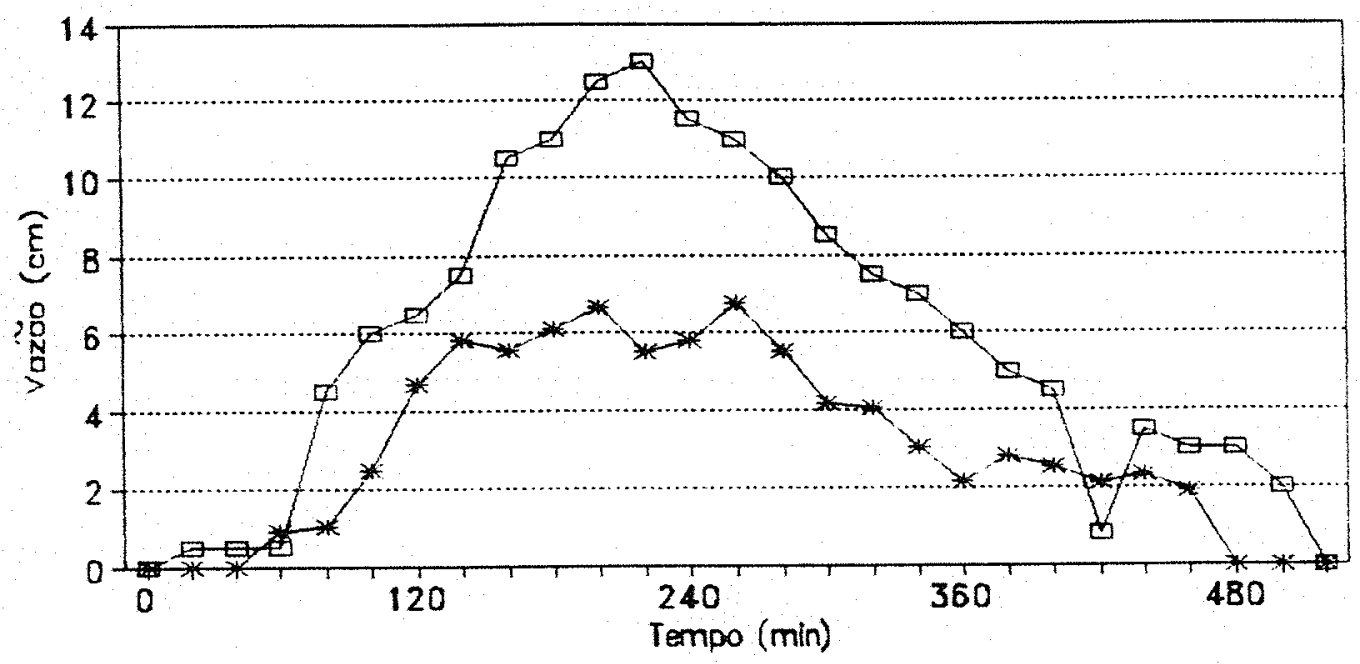

E vazõo observada $*$ vazõo esperada

SIMULACÃO DA HIDRÓGRAFA 37

MICROBACIA DE CUNHA-SP $6 / \mathrm{ABR} / 83 \quad 3 \mathrm{~h} 20^{\circ}$

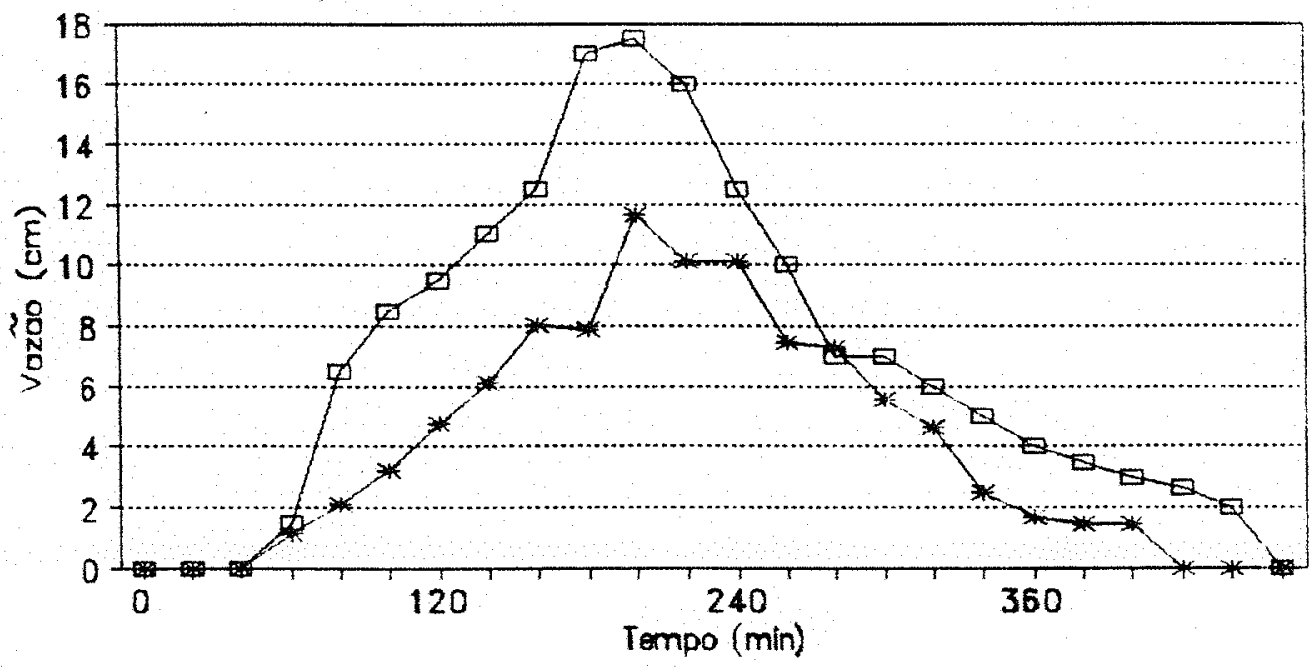

E- vazão observada * vazão esp. KSHA

Figura 44 - Simulaçóes de hidrografas de classe $210 \mathrm{~cm}$ de vazão, com $R a=3.45$ e $R a=3.60$ respecti vamente. 

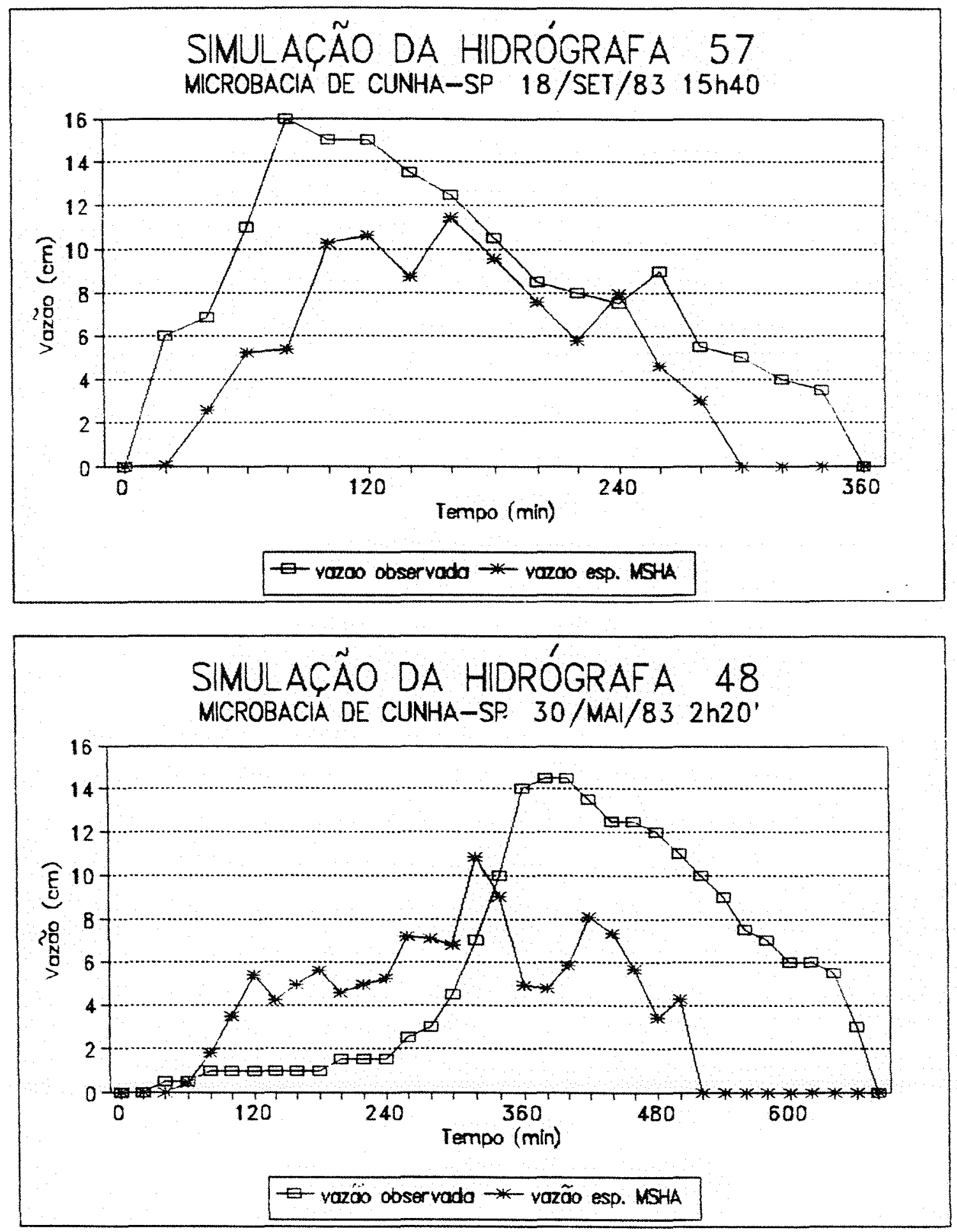

Figura 45 - Simulaçбes de hidrografas de classe $210 \mathrm{~cm}$ de vazão, com $\mathrm{Ra}=2.24$ e $\mathrm{Ra}=5.47$ respecti vamente. 

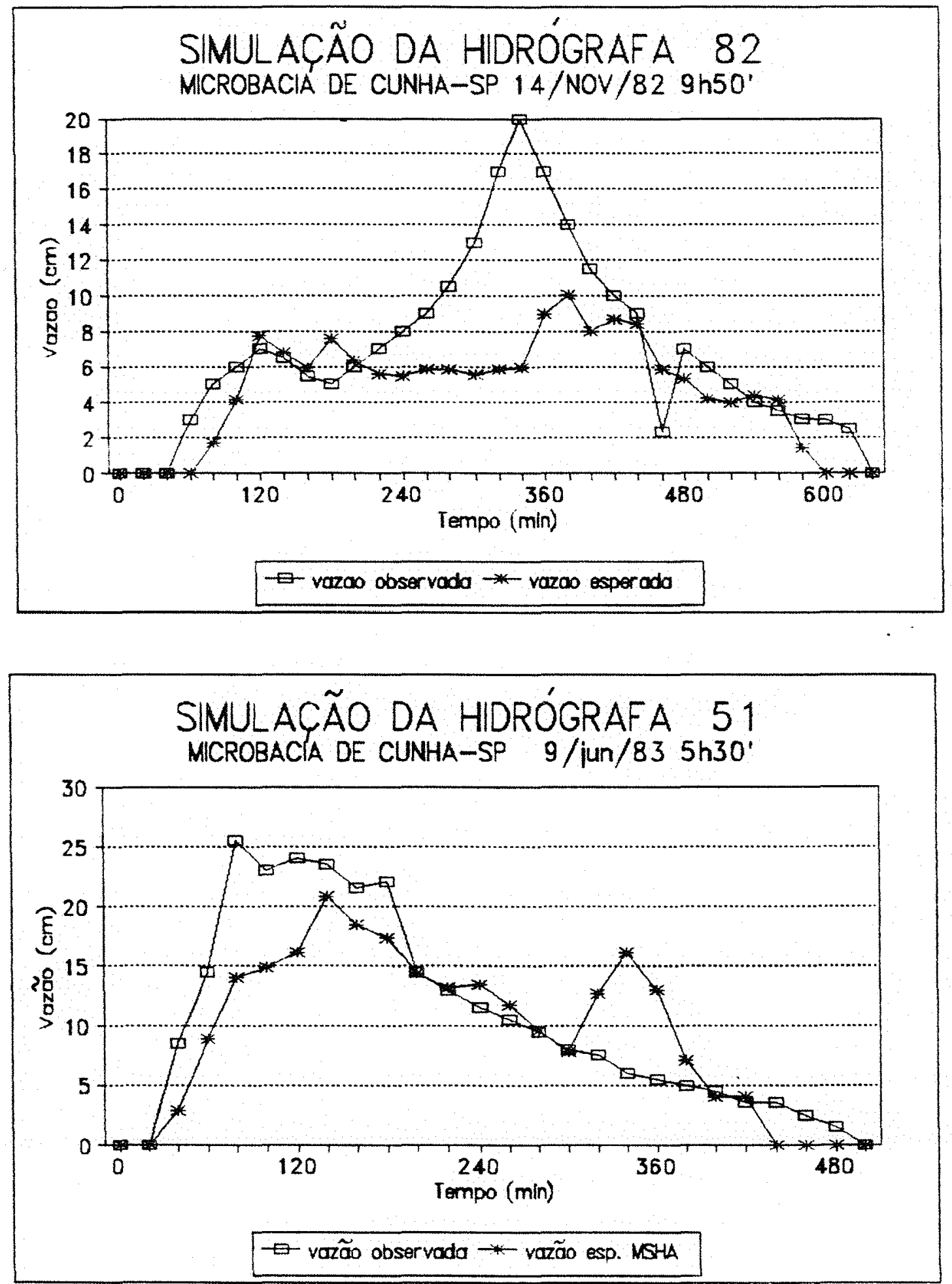

Figura 46 - Simulaçóes de hidrografas de classe $280 \mathrm{~cm}$ de vazão, com $\mathrm{Ra}=4.23$ e $\mathrm{Ra}=4.77$ respecti vamente. 

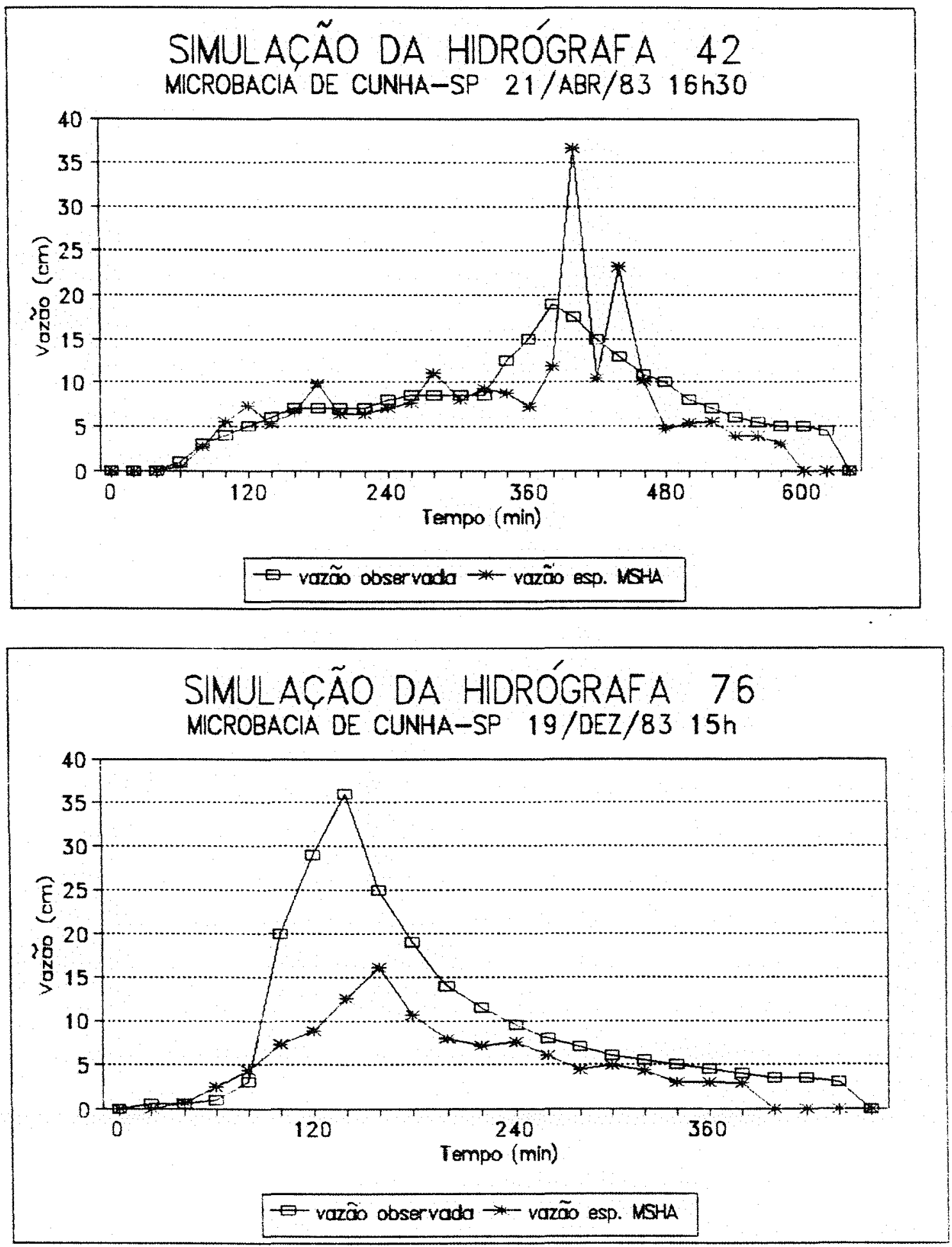

Figura 47 - Simulaçőes de hidrografas de classe $280 \mathrm{~cm}$ de vazão, com $\mathrm{Ra}=4.78$ e $\mathrm{Ra}=7.70$ respecti vamente. 


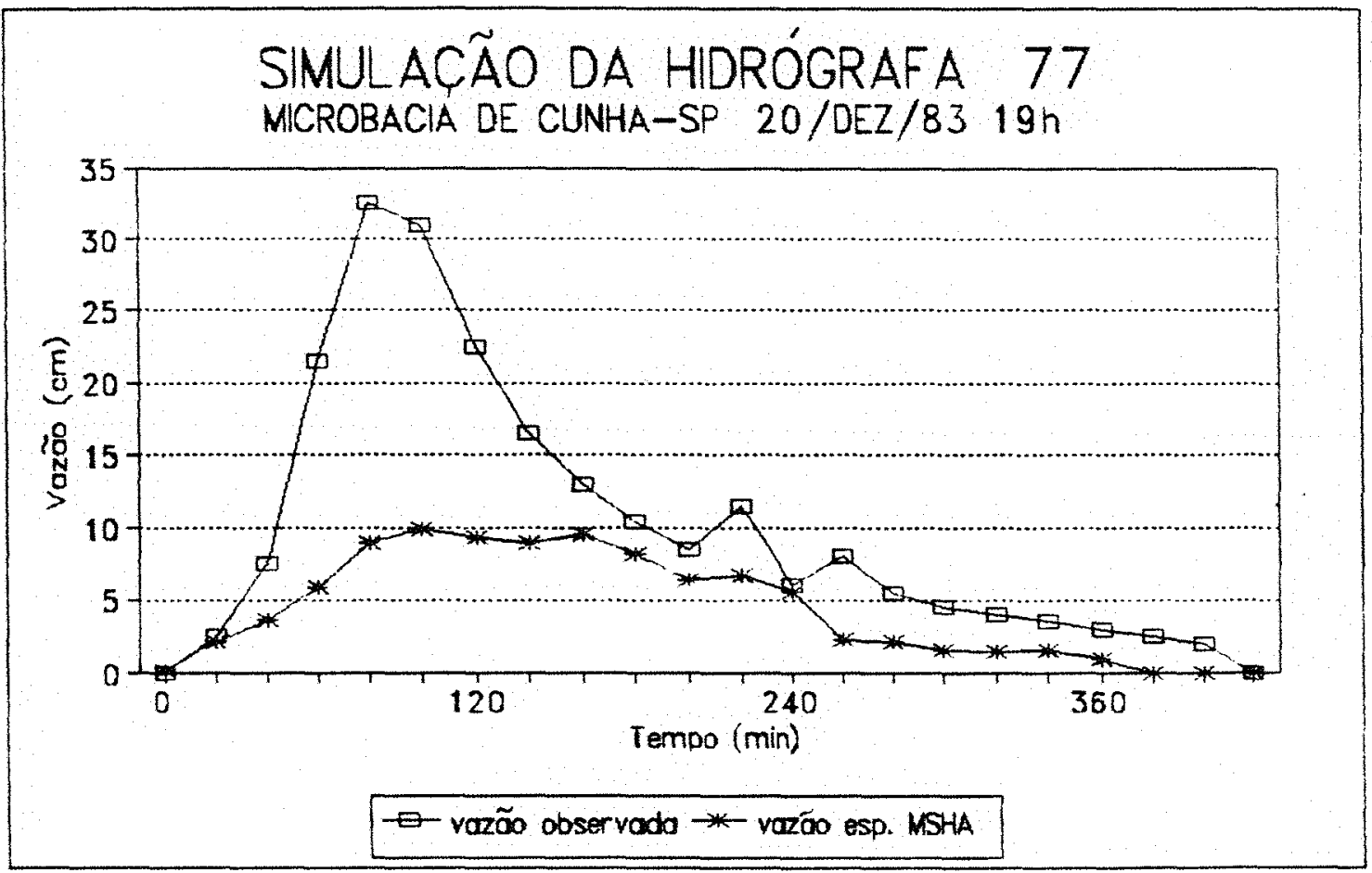

Figura 48 - Simulaçóes de hidrografas de classe $280 \mathrm{~cm}$ de vazão, com $R a=8.50$. 

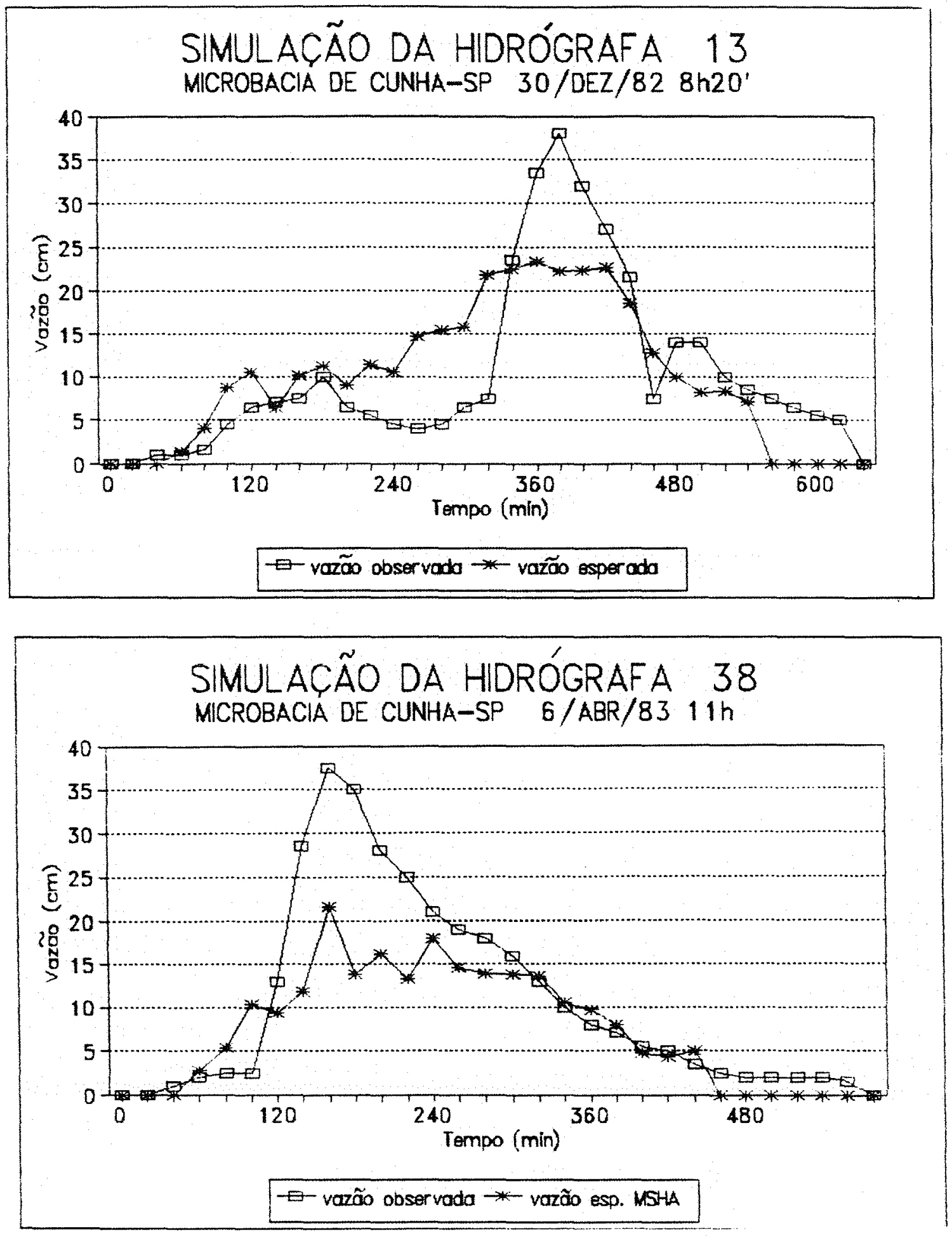

Figura 49 - Simulaçชes de hidrografas de classe $350 \mathrm{~cm}$ de vazão, com $\mathrm{Ra}=6.47$ e $\mathrm{Ra}=6.96$ respecti vamente. 


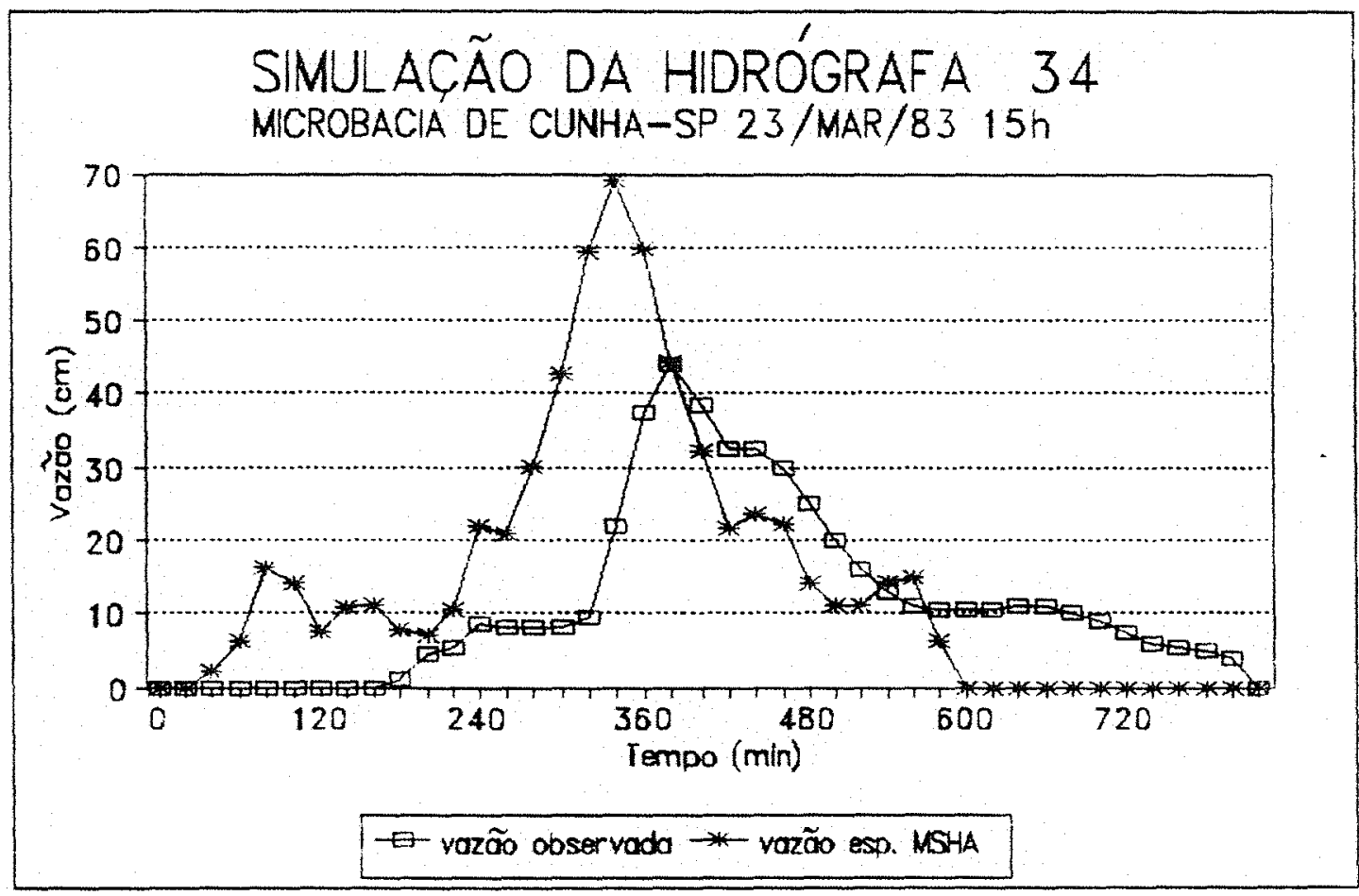

Figura 50 - Simulaçóes de hidrografas de classe $480 \mathrm{~cm}$ de vazáo, com $R_{a}=15.12$. 


\section{CONCLUSÖES}

a) O inicio e fim das hidrografas $(\lambda$ e), parametros de localização, podem ser estimadas conhecendo-se somente - vol ume e o padrå da chuva mais recente chos.

b) O volume da hidrografa $e$ influenciado pelas duas chuvas mais proximas, cho e ch13, atraves das variaveis volume - diferença de tempo entre duas chuvas (tcho1).

c) Podemos utilizar a simulação de hidrografas para previsão de enchentes em baclas hidrograficas, mesmo estimando-se apenas o infcio e fim das hidrografas e o volume total escoado.

d) O modelo MSH ná produziu boas simulaçzes de modo generallzado, tendo como causa a baixa sensibilidade dos parametros de forma do Sistema Johnson $(\delta e \gamma)$ as variaç̧es das chuvas.

e) 0 modelo MSHA produziu boas simulaçరes, sendo que a variavel volume da chuva mais próxima cvolchos foi a mais frequente, com freqútencia de $86.7 \%$, das 34 equaçzes de simul ação das alturas de lámina d'água, acompanhada da varlavel p5ocho (percentil 50 da chuva 0 , com frequencia de ocorrencia de $38.29 \%$.

f) $O$ metodo das distanclas " $\mathrm{Ra}$ " se mostrou adequado para a função de "medir" ajustamentos de hidrografas, sendo possivel ser utilizado para classificar e selecionar ajustamentos de quisquer outras variáveis.

g) Pode-se utilizar o método dos percentis sugerido neste 
trabalho, em qualquer sistema da area de Ciencias Florestais, desde que sejam carcterizados padroes de distribuiçőes de entrada (estimulos), e de salda (respostas).

h) Uma dissertação não se encerra no ato de sua publi cação. Na verdade é neste instante que se inicia uma das principais etapas, a de comunicaça de novas idelas para com a comunidade cientifica. 


\section{REFERENCIAS BIBLIOGRAFICAS}

ABBOTT, M. B. et ali1. An introduction to the European Hydrological System - Systeme Hydrologi que Europeen, "SHE", 1: History \& philosophy of a physically - based, distributed modelling system. Journal of Hydrology, Amsterdam, 87: 45-59, $1986 a$.

ABBOTT, M.B. et alii. An introduction to the European Hydrological System - Systeme Hydrologique Europeen, "SHE", 2: Structure of a physically-based, distributed modelling system. Journal of Hydrology. Amsterdam, 87: $61-77,1986 \mathrm{~b}$.

ALVES, RUBENS Filosofia da Ciecia - introdução ao jogo e suas regras. Ba ed. Sao Paulo, Editora Brasiliense S. A. 1986. 209p.

ANDERSON, M.G. \& BURT, T.P. Modelling strategies. In: ANDERSON, M.G. \& BURT, T.P. Hydrological forecasting. Chichester U.K., John Wiley \& Sons Ltd., 1985. 604p.

ANDO, Y.; MUSI AKE,K. \& TAKAHASI, Y. Modelling of hydrologic processes in a small natural hillslope basin, based on the synthesis of partial hydrological relationships. Journal of Hydrolo8y, Amsterdam, 64: 311-337, 1983.

BARTEN, P. K. \& BROOKS, K. N. Modeling streamflow from headwater areas in the Northern lake states. In: ANDERSON, M. G. \& BURT, T.P. Hydrological Forecasting. Chichester U.K., John Wiley \& Sons Ltd., 1988. 604p.

BATHURST, J.C. Physically-based distributed modelling of an upland catchment using the Systeme Hidrologique Europeen. Journal of Hydrolo8y. Amsterdam, 87: 79-102, $1986 a$. 
BATHURST, J.C. Sensitivity analysis of the Systeme Hidrologique Europeen for an upland catchment. Journal of Hydrolo8y, Amsterdam, 87: 103-123, $1986 \mathrm{~b}$.

BERGSTRON, S. Operational applications of conceptual hydrolical models developed for representative and experimental basins. In: IAHS - International Association of Hydrological Sciences, publication No.130. The influence of man on the hydrological resime with special reference to representative and experimental basins. Helsinki, 1980. 483p.

BERNDTSSON, R. \& NIEMCZYNOWICZ,J. Spatial and temporal scales in rainfall analysis - some aspects and future perspectives. Journal of Hydrolosy, Amsterdam, 100: $293-313,1988$.

BEVEN, K. A sensitivity analysis of the Penman-Montheith actual evapotranspiration estimates. Journal of Hydrology, Amsterdam, 44: 169-190, 1979.

BEVEN, K. Distributed Models. In: ANADERSON, M. G. \& BURT, T.P. Hydrological Forecasting. Chichester U.K., John Wiley \& Sons Ltd. 1985. 604p.

BEVEN, $K$. Changing ideas in hydrology - the case of Physicaly-Based models. Journal of Hydrology. Amsterdam, 105:157-172, 1989.

BEVEN, K. \& WOOD, E.F. Catchment geomorphology and the dynamics of runoff contributing areas. Journal of Hydrolo8y. Amsterdam, 65: 139-158, 1983.

BLACKIE, J.R. \& EELES, L. W. O. Lumped Cathment Models. In: ANDERSON, M. G. \& BURT, T.P. Hydrological forecasting. Chichester U.K. , John Wiley \& Sons Ltd. 1985. $604 p$.

BLIDARU, S.; DRAGOI, E. \& STANCIU, P. Mathematical modelling of overland runoff under various physlographical conditions and for certain agricultural uses. In: IAHS - International Association of Hydrological Sciences, publication No.130. The influence of man on the hydrological regime with special reference to representative and experimental basins. Helsinki, 1980. 483p.

BONELL, $M$. et alii. The generation of storm runoff in a forested clayey drainage basin in Luxembourg. Journal of Hydrology, Amsterdam, 71: 53-77, 1984. 
BORAH, D.K. Runoff simulation model for small watersheds. American Society of Agricultural Engineers, 32(3) may-june, 1989.

BOWMAAN, K.O. \& SHENTON, L.R. Johnson's System of Distributions In: JOHNSON, N.L. \& KOTZ, S. Encyclopedia of Statistical Sciences. 1983.

BOYD, M.J. \& BUFILL, M.C. Determining runoff routing model parameters without rainfall data. Journal of Hydrology, Amsterdam, 108: 281-294, 1989.

BRUI JNZEEL, L. A \& WIERSUM, K.F. Rainfall inteception by a young Acacia auriculiformis (A. Cunn) plantation forest in west Java, Indonesia: application of Gash's analytical model. Hidrological Processes, 1: 309-319. 1987 .

CALDER, I.R. A stochastic model of rainfall interception. journal of Hydrolo8y, Amsterdam, 89: $55-71,1986 a$.

CALDER, I.R.; HRIGHT, I.R. \& MURDIYARSO, D. A study of evaporation from tropical rain forest - west Java. Journal of Hydrolo8y, Amsterdam, 89: 13-31, $1986 \mathrm{~b}$.

CHOW, V.T. Handbook of apllied Hydrology. New York, McGrawhil 1 Book Co., 1964.

CICCO et alii. Determi nação do bal anço hidrico com emprego de bacia hidrografica experimental em mata natural secundaria. In: SIMPOSIO BRASILEIRO DE HIDROLOGIA E RECURSOS HI DRI COS E SIMPOSIO INTERNACI ONAL DE RECURSSOS HI DRI COS EM REGI OES METROPOLITANAS. S. P. NOV. 1985.

CICCO et alii. Determinação da curva-chave do vertedouro da Bacia Hidrográfica Experimental " $D$ " no Parque Estadual da Serra do Mar - Núcleo de Cunha, S. P. Boletim Tecnico do Instituto Florestal, S. P., $41(1): 79-96$, março, 1987.

CICCO et ali1. Interceptação das chuvas por floresta natural secundaria de Mata Atlántica. Silvicultura em São Paulo, 20/22: 25-30, $1986 / 88$.

CLARKE, R. I. Mathematical models in Hydrology. Rome, FAOIrrigation and drainage paper No.19, 1984. 282p.

CLARKE, R. T. The interception process in tropical rain forest: A literature rewiew and critique. Acta Amazonica, 16/17: 225-238, $1986 / 87$. 
DEVILLEZ, F. \& LAUDELOUT, H. Application d'un modele hydrologique à un bassin versant forestier de Wallonie. Ann. Sci. For, 43(4): 475-504, 1986.

DISKIN, M.H.; BURAS, N. \& ZAMIR, S. Application of a simple hydrologic model for rainfall-runof $r$ relations of the Dalton Watershed. Water Resources Research, $9(4)$ : 927-936, aug. 1973 .

DOOGE, J.C. Looking for hydrologic laws. Water Res. Res., 22(9): 46s-58S, aug. 1986.

DRAPER, J. Properties of distributions resulting from certain simple transformation of the Normal distribution. Biometrica, 39: 290-301, 1952.

DRI VER, N. \& TROUTMAN, B.M. Regression models forestimating urbanstorm-rumoff quality and quantity in the United States. Journal of Hydrology, Amsterdam, 109: 221-236, 1989.

DUNNE $T$. Relation of field studies and modeling in the prediction of storm runoff. Journal of Hydrology. Amsterdam 65: 25-48, 1983.

ELDERTON, H.P. \& JOHNSON, N.L. Systems of frequency curves. Cambridge at University Press, 1969.

ESCOLA POLITECNICA DA UNI VERSIDADE DE SAO PAULO. Departamento de Aguas e Energia Eletrica. Associação Brasileira de Hidrologia e Recursos Hidricos. Curso de Engenharia Hidrologica. V.1. São Paulo, 1983.

FEDORA, M. A. \& BESCHTA, R.L. Storm runoff simulation using an atencedent precipitation index (API) model. Journal of Hydrolosy, 112, 121-133, 1989.

FERREIRA, V.A. \& SMITH, R. E. Limited physical basis of physically based Hydrologic Models. In ASAE - American Society of Agricultural Engineers - Publication 07-88. Modeling Agricultural, Forest, and Rangeland Hydrology Proceedings of the 1988 International symposium. Mi chigan U.S.A., 1988. 510p.

FERSI, M. Model of surface runnof in small basins in South Tunisia. In: IAHS - International Association of Hydrological Sciences, publication No. 130 . The influence of man on the hydrological regime with special reference to representative and experimental basins. Helsinki, jun. 1980. 483p. 
FRANKEN, w. et alii. Estudo da interceptacão da água de chuva em cobertura florestal amazonica do tipo terra firme. Acta Amazonica, 12(2): 327-331, 1982.

FRANKEN, W \& LEOPOLDO, P. Hi ydrology of catchment areas of central Amazion Forest streams. In: SIOLI, H. THE AMAZON. LIMINOLOGY AND LANDSCAPE ECOLOGY OF MI GHTY RI VER AND ITS BASTN. Boston, Dr.W. Junk Publishers, 1984.

FREEZE, R. A. A Stochastic - conceptual analysis of rainfall-runoff processes on a hillslope. Water Resources Research, 16(2): 341-408, april 1980.

FURI AN, S.M. \& PFEIFER, R. M. Levantamento de reconhecimento do meio fisico do Núcleo de Cunha - S.P. Boletin Tecnico do Instituto florestal de 5ao Paulo, 40(20) : $183-193$, S. P. dez. 1986.

GASH, J.H. An analitical model of rainfall inteception by forest. Quart. Journal $R$. Met. Soc. 105: 43-55, 1979.

HAGER, W. H. A simplified hydrological rainfall-runoff model. Journal of Hydrolo8y, Amsterdam, 74: 151-170, 1984 .

HAAN, C.T. A water yield model for small watersheds. Water Res. Res. 8(1): 58-91, feb. 1972.

HAAN, C. I. Parametric uncerty in hydrologic modeling. In: ASAE - American Society of Agricultural Engineers No.07-88. Modelling agricultural, forest and rangeland Hydrology - Procedings of the 1988 International Symposium. Michigan U.S.A., 1988. 510 .

HAAN, C.I. \& JOHNSON, H.P. Hydrologic modelling of small watersheds. Michigan, ASAE techinical editor, 1982. 533p. HANSON, C.L. ; OSBORN, H. B.; WOOLHISER, D. A. Dally precipitation simulation model for moutainous areas. Transactions of the ASAE, 32C 3): 865-873, 1989.

HARR, R.D. Water flux in soil and subsoil on a steep forested slope. Journal of Hydrolo8y. Amsterdam, 33: $37-58,1977$.

HEWLETT, J.D. Soil moiture as a source of a base flow from steep mountain watersheds. U.S. Department of Agriculture-Forest Service Station paper no.132. Ashevilie, North Carolina, oct. 1961.

HEWLETT, J.D. Principles of Forest Hydrolosy. The University Press. 183p. 1982. 
HEWLETT, J.D. \& BOSCH,J.M. The dependence of storm flows on rainfall intensity and vegetal cover in South Africa. Journal of Hydrol08y. Amsterdam, 76: 365-381, 1984/1985.

HEHLET, J.D. \& HIBBERT, A.R. Factors affecting the response of small watersheds to precipitation in humid areas. In: SOPPER, W. E. \& LULL, H.W. ed. Affecting the response of small watersheds to precipitation in humid areas, Oxford, Pergamont Press, p. 275-290, 1967.

HI GGINS, R. J. Use and modification of a simple rainfall-runoff model for wet tropical catchments. water Res. Res., 17(2): 423-427, apri1 1981.

HILL, I.D., HILL, R. \& HOLDER, R. L. Fitting Johnson curves by moments. Applied Statistics, 25C2): 180-189, 1976.

HINO, M. \& HASEBE, M. Analysis of hydrologic characteristics from runoff data-A hydrologic inverse problem. Journal of Hydrolo8y, Amsterdam, 49: 287-313, 1981 .

HOLECEK, G. Storage-effecti ve drainage (SED) runoff model. journal of Hydrolo8y. Amsterdam, 98: 295-314, 1988.

ISOTOK, J.D. \& BOERSMA, L. Effect of antecedent rainfall on runoff during - low intensity rainfall. Journal of Hydrolo8y, Amsterdam, 88: 329-342, 1986.

ISOTOK, J.D. \& BOERSMA, L. A stochastic cluster model for hourly precipitation data. Journal of hydrology, Amsterdam, 106: 257-285, 1989.

JACKSON, I. J. Relationships between rainfall parameters and Interception by tropical forest. Journal of Hydrolo8y. Amsterdam, 24: 215-238, 1975.

JOHNSON, N.L. Systems of frequency curves generated by methods of translation. Biometrika, 36: 149-176, 1948.

JOHNSON, N.L. Tables to facilitate fitting Su frequency curves Biometrika, 52(3/4): 547-558, 1965.

JOHNSON, N.L. \& KOTZ, S. Continuous univariate distributions - 2. New York - USA, John Wiley \& Sons, 1970. 306p.

KIRBY, M. Hillslope runoff processes and models. Journal of Hydrolo8y, Amsterdam, 100: $315-339,1989$. 
KLEMES, V. Conceptualization and scale in Hydrology. Jowrnal of Hydroio8y, Amsterdam, 65: 1-23, 1983.

KLEMES, $v$. Dilettantism in Hydrology : transition or destiny? Water Res. Res., 22(9): 1775-1885, 1986.

KLEMES, V. A hydrological prespective. Journal of Hydrolo8y, Amsterdam, 100: 3-28, 1988.

KUHN, T.S. A estrutura das revoluçסes cientificas. STo Paulo, Editora Perspectiva S. A., 1975. 262p.

KUTILEK, M. Flood hydrograph simulation model-A comment. Journal of Hydrolo8y, Amsterdam, 56: 381-382, 1982.

KUUSISTO, K. Conceptual modelining of snowmelt runoff in small drainage basins in Finland. In: IAHS International Association of Hydrological Sciences, publication No.130. The influence of man on the hydrological regime with special reference to representative and experimental basins. Helsinki, 1980. $483 \mathrm{p}$.

LAINE, R.J. Measuring rainfall on forest catchments. Journal of Hydrolo8y, Amsterdam, 9: 103-112, 1969.

LEE, R.P. Forest Hydrolo8y, New York, Columbia Uni versity Press, 1980. 349p.

LEITAO FILHO, P. Aspectos Taxonomicos das florestas do Estado de São paulo. In: CONGRESSO NACIONAL SOBRE ESSENCIAS NATIVAS. Campos do Jordao - S.P. Set. 1982.

LENCASTRE, A. F FRANCO, F.M. Liços de Hidrologia. Portugal, Uni versidade Nova de Lisboa, 1984. $451 \mathrm{p}$.

LEOPOLDO, P.R. et al11. Balanço hidrico de pequena bacia hidrografica amazónica de terra firme. Acta Amazónica $12(2): 333-337.1982$.

LEONARD, R. E. Mathematical teori of interception. In: SOPPER, W. E. \& LULL, H. W. Forest Hydrology, Oxford. Pergamon, 1967 . p. $132-136$.

LESLIE, D.C.M. Determination of parameters in the Johnson System of Probability Distributions. Biometrika, 46: $229-231,1959$. 
LIMA, H.P. Princlpios de hidrologia florestal para o manejo de bacias hidrográficas. Piracicaba, ESALQ. 1986. 242p.

LIMA, W.P. Modelagem do processo de interceptacão. Relatório apresentado ao Departamento de Ciencias Florestais da ESALQ/USP, Viagem ao Instituto de Hidrologia da Inglaterra. Piracicaba, ESALQ, 1987. $64 \mathrm{p}$.

LLOYD, C.R. GASH; J.H.C. \& SHUTTLEWORTH, W. I. The measurement and modelling of rainfall interception by amazonian rain forest. Agricultural and Forest Meteorol08y. 43: 277-294, 1988.

LUNDQUIST, D. Use of a conceptual rainfall - runoff model in water resources planning. In: IAHS - International Association of Hydrological Sciences, publication No. 130 . The influence of man on the hydrological regime with special reference to representative and experimental basins. Helsinki, 1980. 483p.

LUXMOORE, R.J. Infiltration and runoff predictions for a grassland watershed. Journal of Hydrolo8y, Amsterdam, 65: $271-278,1983$.

MAGE, D. T. An explicit solution for Sb parameters using four percentile points. Technometrics, 22(22): 247-251. may 1980 .

MCMAHON, T. A. \& SRIKANTHAN,R. LOg Pearson III distribution - is it applicable to flood frequency analysis of autralian streams? Jornal of Hydrolo8y, Amsterdam, 52: $139-147,1981$.

MARQUES FILHO, A. de 0 et alii. Simulação de escoamento superficial em baclas representativas na Amazónia. Acta Amazonica, $12(3)$ : 29-37, 1982.

MELANEN, M. \& LAUKKANEN, R, Analysis of rainfall - runof $f$ relationships in finnish urban test basins. In: IAHS International Association of Hydrological Sciences, No. 130. The influence of a man on the hydrological regime with special reference to representative and experimental basins. Helsinki, june 1980. 483p. 
MENDEL, 0 . Analysis of a nonlinear rainfall - runoff model and its application to the Mostenik experimental basin in Czechoslovakia. In: IAHS - International Association of Hydrological Sciences, No. 130. The influence of a man on the hydrological regime with special reference to representative and experimental basins. Helsinki, 1980. 483p.

MOSLEY, M. P. Subsurface flow velocities through selected forest solls, South Island, New Zeal and. Journal of Hydrol08y, Amsterdam, 55: 65-92, 1982.

O'LOUGHLIN, E.M. Saturation regions in catchments and their relations to soll and topographic properties. Journal of Hydrology, Amsterdam, 53: 229-246, 1981.

O LOUGHLIN, E. M. Prediction of surface saturation zones in natural catchments by topographic analysis. Water Res. Res. 22(5): 794-804, may 1986.

OGUNKOYA, O. O.; ADEJUWON, J.O. \& JEJE, L.K. Runoff response to basin parameters in Southwestern Nigeria. Jowrnal of Hydrolo8y, Amsterdam, 72: 67-84, 1984.

ORD, J.K. Families of frequency distributions. Grarfins Statistical Mmonographs \& Courses. Hafner Publishing Company N.Y. 1972.

OSBORN, H. B. et alii. Precipitation. In: Haan C. T. et alli, ed. Hydrologic modeling of small watersheds. Asae Monograf No. 5,1982 . $531 \mathrm{p}$.

PEARCE A.J.; STEWART, M.K. \& SKLASH, M. G. Storm runoff generation in humld headwater cat chments. 1 . Where does the water come from? Water Res. Res., 22(8): 1263-1272, aug. 1986.

PESCHKE, G. \& KUTILEK, M. Infiltration model in simulated hydrografs. Journal of Hydrol08y, Amsterdam, 56: $369-379,1982$.

PHIEN, H.N. \& HIRA, M. A. Log Pearson type-3 distribution : parameter estimation. Jornal of Hydrology. Amsterdam, 64: $25-37,1983$.

PHIEN, H.N. \& CHOW, WEN-BIN Applicabllity of the Johnson Sb curve to anual streamflow distribuitions. Jornal of Hydrolo8y, Amsterdam, 64: 15-24, 1983. 
PHIEN, H.N. \& JIVAJIRAJAH, T. Applications of the Log Pearsons Type-3 distribuitions in hydrology. Jornal of Hydrol08y. Amsterdam, 73: 359-372, $1984 a$.

PHIEN, H.N. \& JIVAJIRAJAH, T. Fitting the Sb curve by the method of maximum likelihood. Jornal of Hydrology, Amsterdam, 67: 67-75, $1984 \mathrm{~b}$.

PILGRIM, D. H. et alii. Effects of cathment size on runoff relationships. Journal of Hydrolosy. Amsterdam, 58: 205-221, 1982 .

PILGRIM, D. H. Some problems in transferring hydrological relations between small and large drainage basins and between regions. Journal of Hydrolo8y, Amsterdam, 65: $49-72,1983$.

ROBINSON, $M$. Changes in catchment runoff following drainage and afforestation. Journal of Hydrolosy, Amsterdam, 86: $71-84,1986$.

RODDA, J.C. Basin Studies. In: RODDA, J.C. Facets of Hydrology. vol. 2. New York, John Wiley Sons, 1985.

p. $257-297$.

ROGERSON, T.L. Simulating hydrologic behavior on Ouachita Mountain drainages. US Department of Agriculture Forest Service Research Paper, SO-118, 1976.

RUPRECHT, J.K. \& SCHOFIELD, N.J. Analysis of streamflow generation following deforestation in Southwest Western Australia. Journal of Hydrology, Amsterdam, 105: 1-17, 1989.

RUTTER, A. J.; KERSHAH, K. A. ; ROBINS, P.C. \& MORTON, A. J. A predictive model of rainfall interception in forests, 1. Derivation of the model from observations in a platation of Corsican Pine. Agricultural Meteorol08y, 9: 367-384, $1971 / 1972$.

SHAPIRO, S.S. \& GROSS, A.J. Statistical modeling thechiniques. New York, Marcel Dakker, Inc., 1981. 366p.

SHAW, E. Hydrologic in Practice. Berksheire U.K., Nostrand Reinhold Co. Ltd. . 1983. 569p. 
SKLASH, M. G. ; STEWART, M.K. \& PEARCE, A.J. Storm runof generation in humid headwater catchments. 2 . A case study of hillslope and low-order stream response. Water Res. Res., 22(8): 1273-1282, aug. 1986.

SLIFKER, J.F. \& SHAPIRO, S.S. The Johnson System : Selection and parameter estimation. Technometrics. 22(2): 239-246, may 1980.

SLOAN, P.G. \& MOORE, I.D. Modeling subsurface stormflow on steeply sloping forested watersheds. Water Res. Res. 20(12): 1815-1822, december 1984 .

SRIKANTHAN, R. \& MCMAHON, T. A. LOg Pearson III distribution - Effect of dependence, distribution parameters and sample size on peak annual flood estimates. Jornal of Hydrolo8y, Amsterdam, 52. 149-159, 1981 .

STATISTICAL ANALYSIS SYSTEMS INSTITUTE. User's guide; basics. 5qed. New York, 1985a. 1290 p.

STATISTICAL ANALYSIS SYSTEMS INSTITUTE. User'S guide; statistics. 5ํ.ed. New York, $1985 \mathrm{~b}$. $956 \mathrm{p}$.

TAKASAO, T. \& TAKARA, K. Evaluation of rainfal1-runoff models from the stochastic viewpoint. Journal of Hydrol08y, Amsterdam, 102: $381-406,1988$.

TODINI, E. Rainfal1-Runoff modeling - past, present and future. Journal of Hydrol08y, Amsterdam, 100: $341-352$, 1988.

TSUKAMOTO, Y. \& OHTA, T. Runoff process on a steep forested slope. Journal of Hydrolo8y, Amsterdam, 102: $165-178,1988$.

ULLAH, W. \& BABU, R. Runoff estimates based on rainfall-retention relationship. Indian Forester. 96(2): $127-134,1970$.

WARD, R.C. On the response to precipitation of headwater streams in humld areas. Journal of Hydrology. 74: $171-189,1984 a$.

WARD, R.C. Hypotesis-testing by modeliling catchment response. Journal of Hydrol08y, Ansterdam, 67: 281-305. $1984 \mathrm{~b}$. 
HEEKS, H.D. \& BOUGHTON, W.C. Tests of ARMA Model for rainfall-runoff modelling. Journal of Hydrology, Amsterdam, 91 : $29-47,1987$.

WHEATER, H.S. ; SHAW, T.L. \& RUTHERFORD, J.C. Storm runof small lowl and catchments in Southwest England. Journal of Hydrology, Amsterdam, 55: 321-337, 1982.

WILLIANS, B.J. \& YEH, W.W.G. Parameter estimation in rainfall-runoff models. Journal of Hydrology, Amsterdam, 63: $373-393,1983$.

WISLER, C. O. \& BRATER, E. F. Hidrologia. Rio de Janeiro R. J. AO Livro Tecnico, 1964. 484p.

WOOD, E.F. \& O'CONNELL, P.E. Real time in forecasting. In: Anderson, M. G. \& BURT, T.P. Hydrological Forecasting. Chinchester U.K., John Wiley \& Sons Ltd. . 1985. $604 \mathrm{p}$.

WOOD, E.F. et alii. Effects of spatial variability and scale with implications to hydrologic modeling. Journal of Hydrolosy. Amsterdam, 102: 29-47, 1988.

WOOLHISER, D. A. \& GOODRICH, D.C. Effect of storm rainfall intensity patterns on surface runoff. Jounal of Hydrolo8y, Amsterdam, 102: 335-354, 1988.

WOOLHISER, D. A. \& BRAKENSIEK, D. L. Hydrologic modeling of small watersheds. In: Haan C. $T$. et alii, ed. Hydrologic modeling of small watersheds. Asae Monograf No. $5,1982.531 \mathrm{p}$. 
APÊNDICE 1.

AS CHUVAS 


\begin{tabular}{|c|c|c|c|c|c|c|c|c|c|c|}
\hline \multicolumn{11}{|c|}{ CKUVAS ATUAIS (CHO) USADAS P/SIMULACAO DAS HIDFOGRAFAS ABR-DEZ/82 } \\
\hline CHUVAS- & i & 2 & 3 & 4 & 5 & $\overline{0}$ & 7 & $\overline{8}$ & 8 & 10 \\
\hline DATA $\rightarrow$ & 28ABR & 22010N & 23JUH & $25 J U N$ & 281UN & 18JUL & 10460 & 270UT & $16 N O Y$ & 21DEZ \\
\hline HORA-> & 15 & $7: 20$ & 20 & $0: 20$ & $5: 40$ & 0.30 & $18: 10$ & $17: 30$ & $8: 50$ & 14 \\
\hline $\begin{array}{c}\text { TEWPO } \\
\text { (min) }\end{array}$ & & CHUTAE & & $n \times 2)$ & & & & . & & \\
\hline 0 & n & 0 & 0 & 0 & 0 & 0 & 0 & $\overline{0}$ & 0 & 0 \\
\hline $\mathbf{2 0}$ & 4 & $\mathbf{z}$ & 1 & 5 & 15 & 1 & 5 & 1 & 10 & 3 \\
\hline 40 & 1 & 2 & 1 & 1 & 3 & 4 & 8 & 1 & $\theta$ & 3 \\
\hline 60 & 4 & 0 & 0 & 0 & 3 & 2 & 13 & 3 & 10 & 10 \\
\hline 80 & 3 & 0 & 18 & 0 & 3 & 8 & 8 & 0 & 0 & 1 \\
\hline 100 & 3 & 2 & 1 & 0 & 1 & 7 & 4 & 0 & & 0 \\
\hline 120 & 1 & 1 & 0 & 3 & 3 & 2 & 5 & D & & 0 \\
\hline 140 & 3 & 1 & & 2 & 7 & 15 & $\mathbf{3}$ & 8 & & 1 \\
\hline 160 & 0 & 0 & & 10 & 4 & 5 & 1 & $\overline{8}$ & & 1 \\
\hline 180 & 0 & 0 & & 0 & 4 & 3 & 0 & 0 & & 14 \\
\hline 200 & 2 & 0 & & 0 & 0 & 4 & 1 & 0 & & 4 \\
\hline 220 & 2 & 18 & & 0 & 1 & 2 & 2 & 0 & & 3 \\
\hline 240 & 2 & 5 & & 2 & 0 & 1 & 1 & 7 & & 0 \\
\hline 260 & 3 & 4 & & 3 & & 1 & 1 & 4 & & 0 \\
\hline 280 & 1 & $\theta$ & & 17 & & 0 & 1 & 2 & & 0 \\
\hline 300 & 2 & 4 & & 3 & & 1 & 2 & 2 & & 1 \\
\hline 320 & 2 & 2 & & 1 & & 0 & 1 & 1 & & 0 \\
\hline 340 & 2 & 0 & & 2 & & 2 & 0 & 0 & & \\
\hline 380 & 2 & 1 & & 0 & & 2 & & & & \\
\hline 380 & 2 & & & & & 3 & & & & \\
\hline 400 & 2 & & & & & 1 & & & & \\
\hline 420 & 1 & & & & & 1 & & & & \\
\hline 440 & 1 & & & & & 0 & & & & \\
\hline 460 & 1 & & & & & 1 & & & & \\
\hline 480 & 2 & & & & & 0 & & & & \\
\hline 500 & 2 & & & & & & & & & \\
\hline 520 & 0 & & & & & & & & & \\
\hline 540 & 0 & & & & & & & & & \\
\hline 560 & 0 & & & & & & & & & \\
\hline 560 & 0 & & & & & & & & & \\
\hline 600 & 0 & & & & & & & & & \\
\hline 620 & 0 & & & & & & & & & \\
\hline 640 & 0 & & & & & & & & & \\
\hline 660 & 1 & & & & & & & & & \\
\hline 680 & 1 & & & & & & & & & \\
\hline 700 & 1 & & & & & & & & & \\
\hline 720 & 2 & & & & & & & & & \\
\hline 740 & 2 & & & & & & & & & \\
\hline 760 & 1 & & & & & & & & & \\
\hline 780 & 1 & & & & & & & & & \\
\hline 800 & 1 & & & & & & & & & \\
\hline 820 & 0 & & & & & & & & & \\
\hline 840 & 2 & & & & & & & & & \\
\hline
\end{tabular}




\begin{tabular}{|c|c|c|c|c|c|c|c|c|c|c|}
\hline \multicolumn{11}{|c|}{ CHUVASATUAIS (CHO) USADAS PI SIMULACAO DAS HIDROGRAFAS DEZJANES } \\
\hline CHUNAS- $=$ & 11 & 12 & 13 & 14 & 15 & 16 & 17 & 18 & 19 & 20 \\
\hline DIA -3 & 270EZ & 280E & 3O0EZ & अDEZ & 5JAN & BJAK & QAAN & 12JAN & IWAN & IONAN \\
\hline HORA $\rightarrow$ & 1230 & $15: 40$ & $8: 20$ & 14:10 & $15: 10$ & 1250 & $13: 10$ & 14:10 & $15: 40$ & 11 \\
\hline TEMPO & & & $i^{2} \cdots$ & & & & & & & \\
\hline$($ minin) & & CHIYL & (m & $n \times 2)$ & & & & & & \\
\hline 0 & 0 & 0 & 0 & 0 & $\overline{0}$ & 0 & 0 & 1 & 1 & 1 \\
\hline 20 & 8 & 1 & 1 & 4 & 1 & 7 & 7 & 14 & 15 & 0 \\
\hline 40 & 16 & 10 & 1 & 15 & 0 & 8 & 4 & 2 & $\mathbf{3}$ & 1 \\
\hline 60 & 1 & 16 & 1 & 5 & 1 & 0 & 0 & 0 & 3 & 0 \\
\hline 80 & 0 & 1 & 4 & 1 & 1 & & 0 & 1 & 4 & 0 \\
\hline 100 & & 1 & 6 & 0 & 2 & & 0 & 0 & 3 & 0 \\
\hline 120 & & 2 & 7 & & $i$ & & 0 & & $\overline{\mathbf{u}}$ & 7 \\
\hline 140 & & 1 & 3 & & 2 & & 4 & & 1 & 0 \\
\hline 160 & & 1 & 3 & & 2 & & 16 & & 1 & 0 \\
\hline 180 & & 1 & 0 & & 1 & & 0 & & 0 & 8 \\
\hline 200 & & 1 & 1 & & 1 & & & & 1 & 3 \\
\hline 220 & & 1 & 1 & & 1 & & & & 1 & 3 \\
\hline 240 & & 0 & 0 & & 1 & & & & 0 & 1 \\
\hline 200 & & & 0 & & 0 & & & & 0 & 0 \\
\hline 280 & & & 0 & & 1 & & & & 1 & 0 \\
\hline 300 & & & 5 & & 1 & & & & 0 & 1 \\
\hline 320 & & & 10 & & 1 & & & & 0 & 0 \\
\hline 340 & & & 1 & & 1 & & & & & 2 \\
\hline 360 & & & 30 & & 0 & & & & & i \\
\hline 380 & & & B & & 0 & & & & & 2 \\
\hline 400 & & & 1 & & 1 & & & & & 1 \\
\hline 420 & & & 4 & & 4 & & & & & 2 \\
\hline 440 & & & 2 & & & & & & & 1 \\
\hline 460 & & & 0 & & & & & & & 1 \\
\hline 480 & & & 1 & & & & & & & 0 \\
\hline 500 & & & 0 & & & & & & & 1 \\
\hline 520 & & & & & & & & & & 0 \\
\hline 540 & & & & & & & & & & 0 \\
\hline 560 & & & & & & & & & & 1 \\
\hline 580 & & & & & & & & & & 0 \\
\hline
\end{tabular}




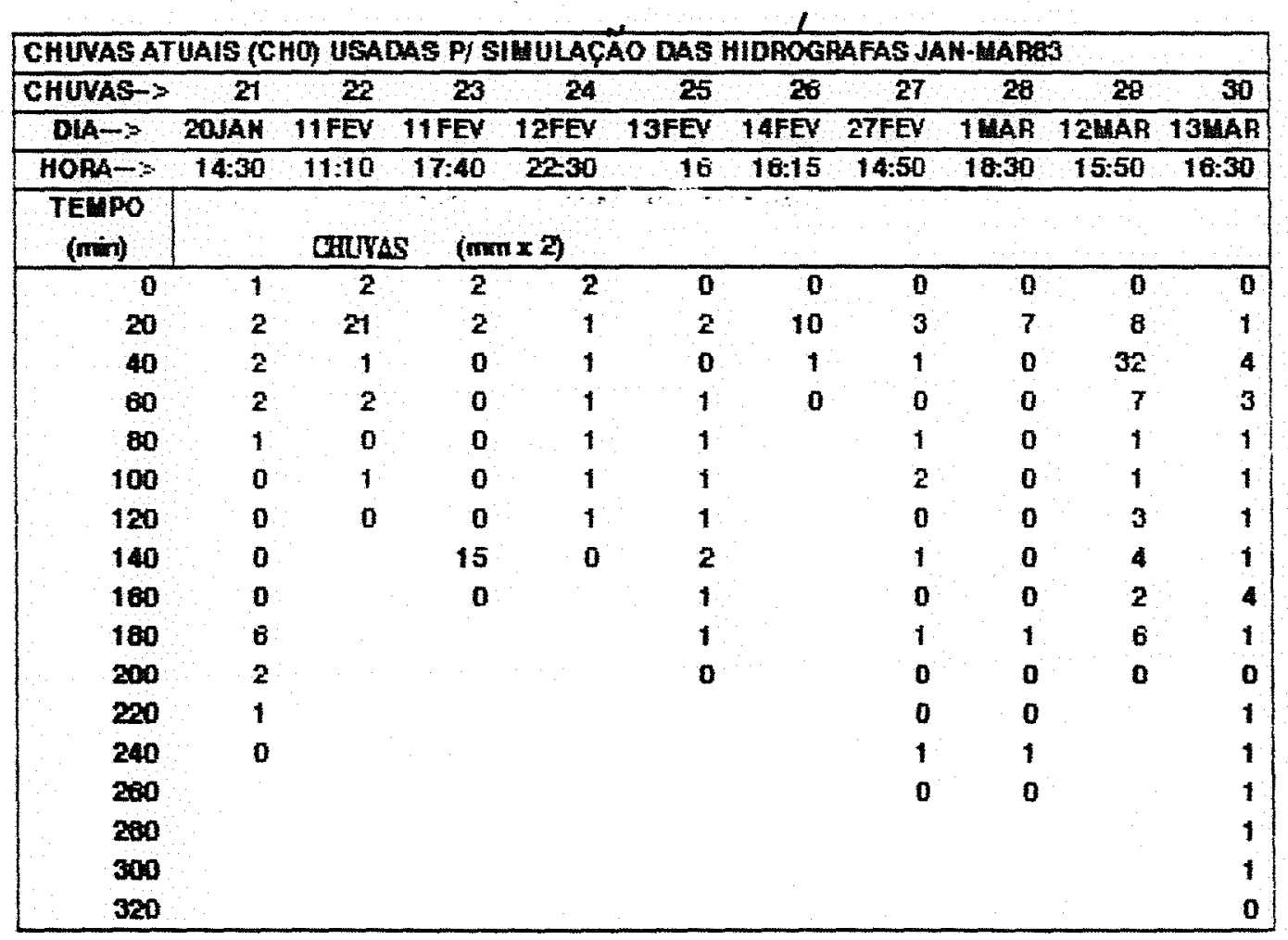




\begin{tabular}{|c|c|c|c|c|c|c|c|c|c|c|}
\hline$\frac{\text { CHUVAS AT }}{\text { CHUNAS-? }}$ & $\frac{\text { UAIS (C) }}{31}$ & H0) USA & $\frac{2 A S P / 5}{35}$ & 34 & 35 & 36 & 37 & 30 & 39 & 40 \\
\hline DIA $\rightarrow$ & IOUAR & 20LAB & 22MAA & Z3МAB & 24UAR & SABR & GABR & 6ABA & $15 \mathrm{ABA}$ & TEABA \\
\hline HORA-O & 16 & $14: 50$ & $15: 30$ & 15 & $15: 10$ & $18: 50$ & $3: 20$ & 11 & 17 & 21:30 \\
\hline $\begin{array}{l}\text { TEMPO } \\
\text { (min) }\end{array}$ & & GHTA & (m & & & & & & & \\
\hline 0 & 0 & 0 & 0 & 0 & $\overline{0}$ & 0 & 0 & 0 & 0 & $\overline{0}$ \\
\hline 20 & 1 & 1 & 1 & 1 & 1 & 1 & 1 & $\theta$ & 9 & 1 \\
\hline 40 & 1 & 0 & 0 & 1 & 0 & 1 & 3 & 3 & 2 & 0 \\
\hline 60 & 2 & 1 & 1 & 0 & 0 & 0 & 9 & 0 & 0 & 1 \\
\hline 80 & 1 & 0 & 1 & 0 & 0 & 3 & $\theta$ & 2 & & 1 \\
\hline 100 & 10 & 1 & 0 & 2 & 1 & 53 & 5 & 24 & & 0 \\
\hline 120 & 10 & 0 & 1 & 1 & 0 & 2 & 2 & 14 & & i \\
\hline 140 & 12 & 0 & 0 & 2 & 0 & g & 4 & 6 & & 2 \\
\hline 160 & 0 & 1 & 1 & 7 & 2 & 2 & 10 & 5 & & 1 \\
\hline 180 & 0 & 0 & 0 & 7 & 2 & 1 & 4 & 3 & & 2 \\
\hline 200 & 0 & 1 & 1 & o & 1 & 3 & 3 & 3 & & 1 \\
\hline 220 & 1 & 0 & 0 & 0 & 0 & 0 & 0 & 3 & & 1 \\
\hline 240 & 2 & 1 & & 7 & 2 & 1 & & 5 & & 1 \\
\hline 260 & 4 & 1 & & 0 & 0 & 0 & & 1 & & 2 \\
\hline 280 & 1 & 1 & & 0 & & & & 0 & & 1 \\
\hline 300 & 1 & 1 & & $\theta$ & & & & & & 1 \\
\hline 320 & 5 & 1 & & 20 & & & & & & 0 \\
\hline 340 & 3 & 1 & & 9 & & & & & & 1 \\
\hline 300 & 1 & 0 & & 7 & & & & & & 1 \\
\hline 390 & 2 & 0 & & 4 & & & & & & 1 \\
\hline 400 & 1 & 1 & & 7 & & & & & & 1 \\
\hline 420 & 2 & 0 & & 5 & & & & & & 1 \\
\hline 440 & 2 & 0 & & 1 & & & & & & 0 \\
\hline 460 & 0 & 0 & & 0 & & & & & & 0 \\
\hline 480 & 1 & 1 & & 0 & & & & & & 0 \\
\hline 500 & 1 & 0 & & 0 & & & & & & 1 \\
\hline 520 & 1 & 0 & & 2 & & & & & & 0 \\
\hline 540 & 0 & & & 2 & & & & & & 1 \\
\hline 560 & & & & 0 & & & & & & 0 \\
\hline 580 & & & & 1 & & & & & & \\
\hline 600 & & & & 8 & & & & & & \\
\hline 620 & & & & 0 & & & & & & \\
\hline 840 & & & & 0 & & & & & & \\
\hline 660 & & & & 0 & & & & & & \\
\hline 680 & & & & 1 & & & & & & \\
\hline 700 & & & & 0 & & & & & & \\
\hline
\end{tabular}




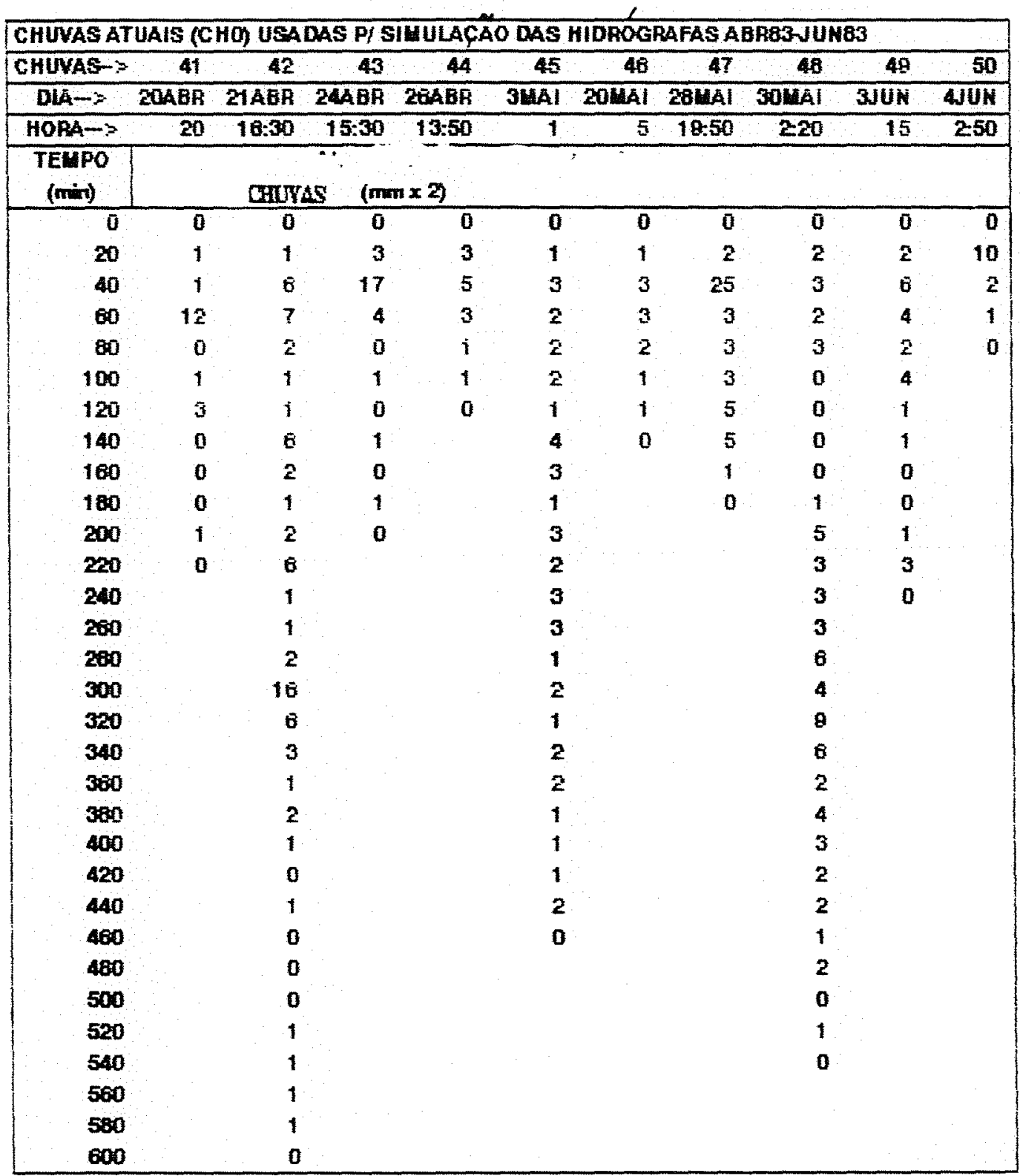




\begin{tabular}{|c|c|c|c|c|c|c|c|c|c|c|}
\hline \multicolumn{11}{|c|}{ CHUVAS ATUAIS (CHO) USADAS P/ SIHULACAO DAS HIDROGRAFAS JUNO3-GET83 } \\
\hline CHUVAS-? & 51 & 52 & 53 & 54 & 55 & 56 & $\mathbf{5 7}$ & 58 & 59 & 60 \\
\hline DLA $\rightarrow=$ & DUN & 28.JUK & $18 \mathrm{NUL}$ & $2 A G O$ & AAGO & 10SET & 18SET & $18 S E T$ & 22SET & 23SET \\
\hline HORA-S & $5: 30$ & 15:50 & $5: 50$ & $\mathbf{2 3 : 5 0}$ & $7: 40$ & 230 & $15: 40$ & $14: 20$ & 13:55 & $3: 50$ \\
\hline $\begin{array}{c}\text { TEUPO } \\
\text { (min) }\end{array}$ & & CHTS & $15 \quad \mathrm{~m}$ & (x) & & & & & & \\
\hline 0 & $\overline{0}$ & $i$ & 0 & $\overline{\mathbf{u}}$ & $\overline{\mathbf{0}}$ & $\overline{0}$ & 0 & $\overline{0}$ & $\overline{0}$ & $\overline{0}$ \\
\hline 20 & 35 & 36 & 1 & 1 & 1 & 5 & 34 & 1 & 4 & 2 \\
\hline 40 & $g$ & 2 & 5 & $\theta$ & 0 & 10 & $\bar{\sigma}$ & 3 & 0 & 2 \\
\hline 60 & 15 & 12 & 2 & 2 & 1 & 2 & 4 & 4 & 4 & 2 \\
\hline 80 & 7 & 10 & $\mathbf{i}$ & 3 & 1 & 0 & 4 & $\bar{z}$ & 8 & 2 \\
\hline 100 & 8 & 2 & 2 & 1 & 1 & 0 & 3 & 1 & 11 & 2 \\
\hline 120 & 4 & 2 & 0 & 2 & 1 & 0 & 3 & 0 & 0 & 3 \\
\hline 140 & 0 & 3 & 0 & 1 & 2 & 1 & 1 & 0 & 0 & 0 \\
\hline 160 & 1 & $f$ & 0 & 1 & 1 & 5 & 0 & 0 & 4 & 3 \\
\hline 180 & 4 & $\mathbf{2}$ & 1 & 0 & 1 & 2 & & 0 & 12 & 0 \\
\hline 200 & 1 & 1 & 1 & 1 & i & 0 & & $\mathbf{i}$ & 3 & \\
\hline 220 & 1 & 0 & 0 & 0 & 1 & & & 0 & 3 & \\
\hline 240 & 0 & & 1 & 2 & 1 & & & 1 & 2 & \\
\hline 260 & 0 & & $\hat{2}$ & 1 & 0 & & & 0 & 1 & \\
\hline 280 & & & 0 & 5 & $i$ & & & 0 & 1 & \\
\hline 300 & & & 2 & 0 & 0 & & & 1 & 0 & \\
\hline 320 & & & 1 & & & & & 1 & 1 & \\
\hline 340 & & & 3 & & & & & 0 & 0 & \\
\hline 360 & & & 1 & & & & & & & \\
\hline 380 & & & 3 & & & & & & & \\
\hline 400 & & & 2 & & & & & & & \\
\hline 420 & & & 2 & & & & & & & \\
\hline
\end{tabular}




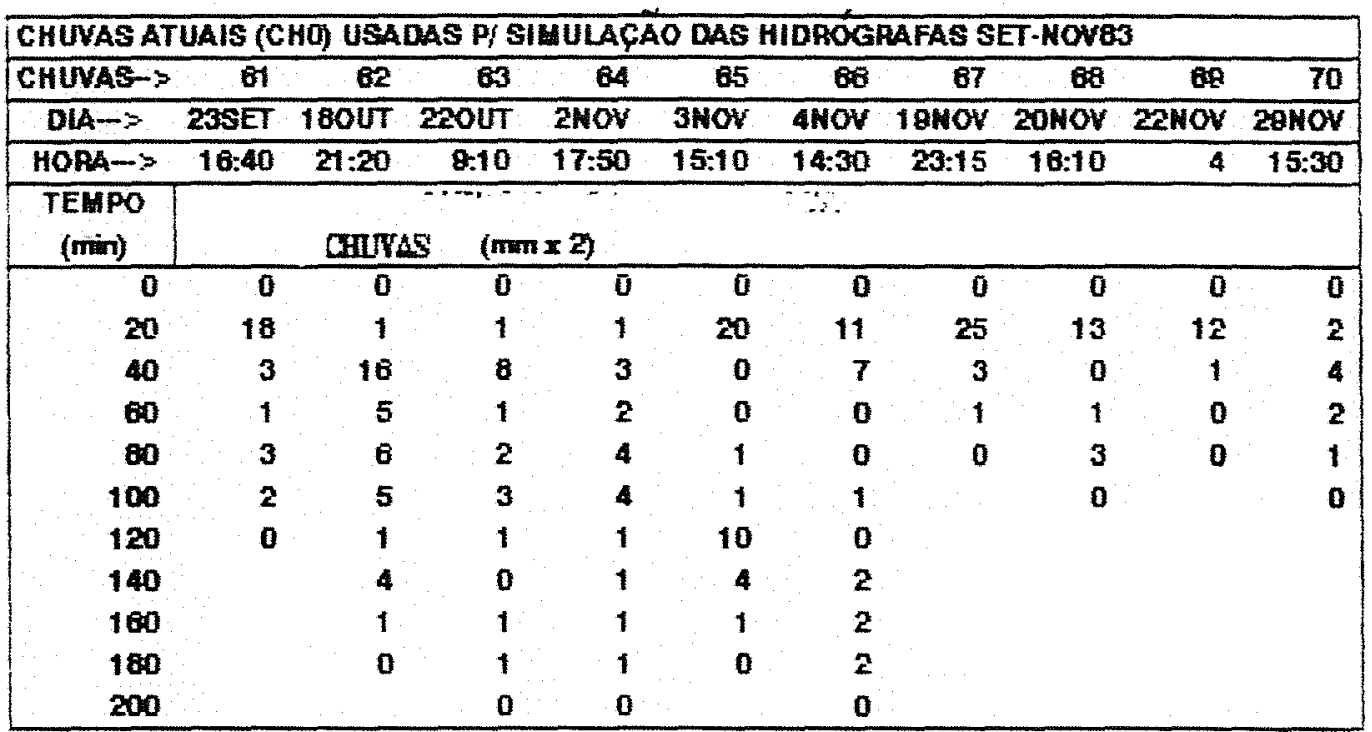

\begin{tabular}{|c|c|c|c|c|c|c|c|c|c|c|c|c|}
\hline \multicolumn{13}{|c|}{ CHUVAS ATUAIS (CHO) USADAS } \\
\hline CHUVAS-P & 71 & 72 & 73 & 74 & 75 & 76 & 77 & 78 & 78 & +80 & 61 & +82 \\
\hline DIA-? & 1DEZ & 10DEZ & 16DEZ & $170 E$ & 180EZ & 18DEZ & 2ODEZ & 25DEZ & 25DEZ & $2900 T$ & 290UT & $14 \mathrm{HOV}$ \\
\hline HORA-3 & $15: 20$ & 15:50 & 14 & $17: 35$ & $19: 30$ & 15 & 19 & 210 & $7: 50$ & 5 & $13: 30$ & 9.50 \\
\hline $\begin{array}{c}\text { TEYPO } \\
\text { (min) }\end{array}$ & & MUTA & (m) & $\mathbf{n \times 2})$ & & & & & & & & \\
\hline $\mathbf{0}$ & $\mathbf{0}$ & 0 & 0 & 0 & 0 & $\mathbf{0}$ & 0 & 0 & $\mathbf{0}$ & 0 & 0 & 0 \\
\hline 20 & 7 & 4 & 1 & 8 & 2 & 4 & 14 & 1 & 4 & 5 & 5 & 4 \\
\hline 40 & 6 & 4 & 10 & 11 & 7 & 1 & 7 & 18 & 14 & 3 & 3 & 8 \\
\hline 60 & 20 & 5 & 0 & 1 & 11 & 4 & 22 & 13 & 3 & 24 & 0 & 5 \\
\hline 80 & 10 & 0 & 1 & 0 & 3 & 10 & 3 & 2 & 3 & 7 & 0 & 1 \\
\hline 100 & $\theta$ & & 0 & 1 & 1 & 28 & 2 & 3 & 1 & 2 & 0 & 0 \\
\hline 120 & 1 & & & 0 & 1 & 11 & 0 & 2 & 1 & 0 & 0 & 0 \\
\hline 140 & 0 & & & 1 & 0 & 1 & & 0 & 0 & 1 & 0 & 1 \\
\hline 160 & & & & 0 & & 0 & & & & 0 & 16 & 1 \\
\hline 180 & & & & & & & & & & & 0 & 1 \\
\hline 200 & & & & & & & & & & & 0 & 12 \\
\hline 220 & & & & & & & & & & & 0 & 3 \\
\hline 240 & & & & & & & & & & & 0 & 1 \\
\hline 200 & & & & & & & & & & & 8 & 4 \\
\hline 280 & & & & & & & & & & & 2 & 4 \\
\hline 300 & & & & & & & & & & & 0 & $\theta$ \\
\hline 320 & & & & & & & & & & & 1 & 5 \\
\hline 340 & & & & & & & & & & & 17 & $\theta$ \\
\hline 360 & & & & & & & & & & & 1 & 3 \\
\hline 380 & & & & & & & & & & & 2 & 2 \\
\hline 400 & & & & & & & & & & & 2 & 0 \\
\hline 420 & & & & & & & & & & & 2 & 0 \\
\hline 440 & & & & & & & & & & & 0 & 3 \\
\hline 460 & & & & & & & & & & & 0 & 1 \\
\hline 480 & & & & & & & & & & & 1 & 0 \\
\hline 500 & & & & & & & & & & & 0 & 1 \\
\hline $\mathbf{5 2 0}$ & & & & & & & & & & & & 0 \\
\hline
\end{tabular}


APÊNDICE 2

AS HIDRÓGRAFAS 


\begin{tabular}{|c|c|c|c|c|c|c|c|c|c|c|}
\hline \multicolumn{11}{|c|}{ HIDROGRAFAS UTILIZADAS PARA SMMULACAO ABR/82 - DEZ/82 } \\
\hline HIDROAS-> & 1 & 2 & 3 & 4 & 5 & 6 & 7 & 8 & 9 & 10 \\
\hline DATA $\rightarrow$ & 28ABR & $220 \mathrm{~N}$ & 23run & 25JuN & $265 \mathrm{UN}$ & 19:UL & 10400 & $270 U T$ & 1arov & $210 E 2$ \\
\hline HORA $\rightarrow$ & 15 & $7: 20$ & 20 & 0.20 & $5: 40$ & 0.30 & $18: 10$ & 1730 & 8.50 & 14 \\
\hline $\begin{array}{c}\text { TPMPO } \\
\text { (min) }\end{array}$ & \multicolumn{10}{|c|}{$\begin{array}{l}\text { ALTURAS DE LAMNA D'AGUA } \\
(\mathrm{cm} 2)\end{array}$} \\
\hline 0 & 0 & 0 & 0 & 0 & 0 & 0 & 0 & 0 & 0 & 0 \\
\hline 20 & 0 & 0 & 0 & 0 & 0 & 0 & 0 & 0 & 15 & 0 \\
\hline 40 & as & 0 & 0 & 0 & 0 & 0 & 0.5 & 0 & 20 & 0 \\
\hline 60 & 1.0 & 0 & 0 & 0 & os & 0 & 15 & 0 & 5.5 & 20 \\
\hline 80 & 1.0 & 0 & 1.0 & 0 & as & 0 & 20 & 0 & 65 & 25 \\
\hline 100 & 15 & 0 & 1.0 & 0 & as & 0 & 25 & 0 & 5.5 & 25 \\
\hline 120 & 20 & 0 & 1.0 & 0 & 1.0 & 0 & 3.0 & 1.0 & 5.0 & 20 \\
\hline 140 & 2.5 & 0 & 2.0 & 0 & 1.5 & a.s & 3.0 & 1.0 & 5.5 & 2.5 \\
\hline 160 & 2.5 & 0 & 1.0 & 0 & 25 & 1.5 & 3.0 & 15 & 5.0 & 20 \\
\hline 180 & 25 & 0 & & 0 & 3.0 & 25 & 3.0 & 1.5 & 4.0 & 4.0 \\
\hline 200 & 25 & 0 & & 0 & 3.0 & 25 & 30 & 1.5 & 3.5 & 7.5 \\
\hline 20 & 25 & 1.0 & & 0 & 3.0 & 25 & 25 & 1.5 & 30 & 7.0 \\
\hline 240 & 2.5 & 20 & & 0 & 30 & 25 & 25 & 20 & 25 & 65 \\
\hline 260 & 2.5 & 20 & & 0 & 3.0 & 25 & 20 & 25 & 25 & 65 \\
\hline 200 & 25 & 3.0 & & 25 & 25 & 25 & 20 & 25 & 20 & 5.5 \\
\hline 300 & 3.0 & 3.5 & & 5.0 & 20 & 20 & 20 & 25 & 20 & 5.0 \\
\hline 320 & 30 & 3.5 & & 7.5 & 20 & 20 & 20 & 25 & 20 & 40 \\
\hline 340 & 35 & 3.0 & & 7.0 & 1.5 & 15 & 20 & 25 & 1.5 & 30 \\
\hline 360 & 35 & 25 & & 5.0 & 15 & 1.0 & 20 & 20 & 1.5 & 25 \\
\hline 300 & 40 & 20 & & 35 & 15 & 10 & 20 & 20 & 15 & 20 \\
\hline 400 & 40 & 20 & & 25 & 1.0 & 1.0 & 20 & 20 & 2.0 & 20 \\
\hline 420 & 4.0 & 1.5 & & 20 & 1.0 & 1.0 & 20 & 20 & & 20 \\
\hline 440 & 40 & 1.5 & & 1.5 & 1.0 & 1.0 & 20 & 20 & & 1.5 \\
\hline 460 & 40 & 1.0 & & 1.0 & 1.0 & 1.0 & 15 & 20 & & 1.5 \\
\hline 480 & 40 & 1.0 & & 1.0 & 0.5 & 1.0 & & 15 & & 1.5 \\
\hline 500 & 40 & 1.0 & & 1.0 & 0 & 1.0 & & 1.5 & & 1.0 \\
\hline 520 & 40 & 10 & & as & & 1.0 & & 1.5 & & \\
\hline 540 & 40 & 2.0 & & as & & 1.0 & & 1.5 & & \\
\hline 560 & 4.5 & 1.0 & & & & 1.0 & & 15 & & \\
\hline 580 & 3.0 & 1.0 & & & & 1.0 & & 1.5 & & \\
\hline 600 & 30 & 1.0 & & & & 1.0 & & 15 & & \\
\hline 600 & 30 & 1.0 & & & & as & & 15 & & \\
\hline 640 & 30 & as & & & & as & & 1.5 & & \\
\hline 660 & 3.13 & 0 & & & & as & & 1.5 & & \\
\hline 680 & 30 & & & & & as & & 1.5 & & \\
\hline 700 & 3.0 & & & & & 0.5 & & 1.5 & & \\
\hline 720 & 3.0 & & & & & 0.5 & & 1.5 & & \\
\hline 740 & 30 & & & & & as & & 1.5 & & \\
\hline 760 & 3.0 & & & & & as & & 1.5 & - & \\
\hline 780 & 0.0 & & & & & as & & 1.0 & & \\
\hline 800 & a. & & & & & 0 & & 0 & & \\
\hline 820 & 1.5 & & & & & & & & & \\
\hline 840 & 0.0 & & & & & & & & & \\
\hline
\end{tabular}




\begin{tabular}{|c|c|c|c|c|c|c|c|c|c|c|}
\hline \multicolumn{11}{|c|}{ HIDROGRAFAS UTILIZADAS PARA SIMULACAO DEZ/82 - IAN/83 } \\
\hline HIDROGS $>$ & 11 & 12 & 13 & 14 & 15 & 16 & 17 & 38 & 19 & 20 \\
\hline $\mathrm{DLA} \rightarrow$ & $2 \mathrm{DDEZ}$ & $29 \mathrm{DEZ}$ & 3ODEZ & $31 \mathrm{DEZ}$ & SJAN & 8JAN & 9JAN & $12 \mathrm{JAN}$ & 13JAN & 19IAN \\
\hline HORA $\rightarrow$ & 1230 & $15: 40$ & $8: 20$ & 1410 & $15: 10$ & 12.50 & $13 \times 10$ & $14: 10$ & $15: 40$ & 11 \\
\hline $\begin{array}{c}\text { TEMPO } \\
\text { (min) }\end{array}$ & \multicolumn{10}{|c|}{$\begin{array}{l}\text { ALTUKAS DE LAMANA DAGUA } \\
\left(\mathrm{cm}^{\circ} 2\right)\end{array}$} \\
\hline 0 & 0 & 0 & 0 & 0 & 0 & 0 & 0 & 0 & 0 & 0 \\
\hline 20 & as & 0 & 0 & 1.0 & 0 & 10 & 0.5 & 0 & 0 & 0 \\
\hline 40 & 4.5 & 1.0 & 10 & 25 & 0 & 25 & 1.0 & 1.5 & 25 & 0 \\
\hline 60 & 60 & 5.5 & 1.0 & 40 & 0 & 35 & 1.0 & 25 & 5.5 & 0 \\
\hline 80 & 45 & 7.0 & 15 & 45 & 0 & 3.0 & 1.0 & 3.0 & 50 & 0 \\
\hline 100 & 40 & 8.0 & 4.5 & 40 & 0 & 3.0 & 1.0 & 25 & s.o & 0 \\
\hline 120 & 3.0 & 5.5 & 6.5 & 3.5 & as & 3.5 & as & 4.0 & 8.0 & 0 \\
\hline 140 & 3.0 & 60 & 7.0 & 3.0 & 1.0 & 20 & 1.5 & 3.0 & 7.5 & 0.5 \\
\hline 160 & 25 & 5.5 & 75 & 25 & 15 & 1.5 & 5.5 & 25 & 6.5 & 10 \\
\hline 180 & 20 & 5.0 & 100 & 20 & 1.5 & 1.5 & 5.5 & 20 & 5.5 & 10 \\
\hline 200 & 1.5 & 4.5 & 6.5 & 20 & 1.5 & 1.0 & 3.5 & 1.5 & 5.0 & 15 \\
\hline 220 & 1.5 & 40 & 5.5 & 15 & 20 & 10 & 25 & 15 & 45 & 20 \\
\hline 240 & 1.0 & 3.5 & 4.5 & 1.5 & 20 & as & 20 & 15 & 4.0 & 25 \\
\hline 260 & & 3.0 & 40 & 1.5 & 20 & & 20 & 1.0 & 3.5 & 25 \\
\hline 280 & & 3.0 & 4.5 & 1.0 & 20 & & 1.5 & & 3.0 & 25 \\
\hline 300 & & 30 & 6.5 & 1.0 & 20 & & 1.5 & & 25 & 25 \\
\hline 320 & & 3.0 & 7.5 & as & 20 & & 1.0 & & 25 & 20 \\
\hline 340 & & 25 & 235 & & 20 & & 1.0 & & 20 & 20 \\
\hline 360 & & 25 & 335 & & 20 & & 10 & & 20 & 20 \\
\hline 380 & & 20 & 380 & & 20 & & 2.0 & & 1.5 & 20 \\
\hline 400 & & 20 & 320 & & 1.5 & & 0.5 & & 15 & 20 \\
\hline 420 & & 20 & 27.0 & & 1.5 & & & & 1.5 & 20 \\
\hline 440 & & 20 & 21.5 & & 3.5 & & & & 1.0 & 20 \\
\hline 400 & & 1.5 & 7.2 & & 1.5 & & & & 100 & 25 \\
\hline 480 & & 1.5 & 140 & & 15 & & & & 1.0 & 25 \\
\hline 500 & & 1.5 & 140 & & 1.0 & & & & as & 25 \\
\hline 520 & & 1.5 & 100 & & 2.0 & & & & & 25 \\
\hline 540 & & 1.0 & 8.5 & & 1.0 & & & & & 25 \\
\hline 560 & & 0 & 7.5 & & 1.0 & & & & & 20 \\
\hline 580 & & & 65 & & 1.0 & & & & & 20 \\
\hline$\infty$ & & & 5.5 & & 1.0 & & & & & 15 \\
\hline$\infty 0$ & & & 5.0 & & 1.0 & & & & & 15 \\
\hline 640 & & & 0 & & 1.0 & & & & & 15 \\
\hline 660 & & & & & 1.0 & & & & & 1.0 \\
\hline 680 & & & & & 1.0 & & & & & 0 \\
\hline 700 & & & & & a.s & & & & & \\
\hline no & & & & & 0 & & & & & \\
\hline
\end{tabular}




\begin{tabular}{|c|c|c|c|c|c|c|c|c|c|c|}
\hline \multicolumn{11}{|c|}{ HWROGRAFAS UTUUZADAS PARA SIMULACAO JAN/B3 - MAR/O3 } \\
\hline HIDROGS $>$ & 21 & 22 & 23 & 24 & 25 & 26 & 27 & 28 & 29 & 30 \\
\hline DUA $\rightarrow$ & 2OIAN & 11FEY & 11FEY & $12 \mathrm{FEV}$ & 13PEV & 14FEV & 27FEV & IMAR & I2MAR & 13MAR \\
\hline HORA $\rightarrow$ & $14: 30$ & $21: 10$ & $17: 40$ & $22: 30$ & 16 & $16: 25$ & 2450 & 1030 & $15: 30$ & $16 \times 30$ \\
\hline $\begin{array}{c}\text { TEMPO } \\
\text { (mia) }\end{array}$ & \multicolumn{10}{|c|}{$\begin{array}{l}\text { ALTURAS DELAMINA D'AGUA } \\
\left(\mathrm{am}^{*} 2\right)\end{array}$} \\
\hline 0 & 0 & 0 & ( & 0 & 0 & 0 & 0 & 0 & 0 & a \\
\hline 20 & 0 & 0 & 0 & 0 & 0 & 1.5 & 1.0 & 1.0 & 20 & 0 \\
\hline 40 & 0 & 0 & 10.0 & 0 & 0 & 1.5 & 1.0 & 1.0 & 7.5 & 0.5 \\
\hline 60 & as & 0 & 18.0 & 1.0 & as & 3.0 & 10 & 20 & 125 & as \\
\hline$\infty$ & a.s & 0 & 160 & 1.0 & 0.5 & 3.0 & 20 & 25 & 16.5 & 1.0 \\
\hline 100 & 1.0 & 0.5 & 23.0 & 1.5 & 0.5 & 4.0 & 5.5 & 25 & 13,0 & 1.5 \\
\hline 120 & 1.0 & 0.5 & 16.0 & 3.0 & 1.0 & 3.0 & 8.5 & 25 & 11.0 & 1.5 \\
\hline 140 & 1.0 & 0.5 & 11.0 & 3.0 & 10 & 20 & 70 & 25 & 8.5 & 15 \\
\hline 160 & 1.0 & 25 & 9.0 & 3.5 & 15 & 1.5 & 65 & 25 & 7.5 & 25 \\
\hline 180 & 1.0 & 25 & 6.5 & 3.5 & 1.5 & 1.5 & 65 & 30 & 80 & 25 \\
\hline 200 & 15 & 25 & 5.5 & 35 & 20 & 10 & 5.5 & 35 & B. 0 & 35 \\
\hline 220 & 20 & 20 & 15 & 3.0 & 25 & 1.0 & 4 & 40 & 7.5 & 3.5 \\
\hline 240 & 25 & 20 & 40 & 25 & 25 & 2.0 & 4.0 & 4.0 & 7.0 & 3.5 \\
\hline 250 & 20 & 20 & 3.0 & 20 & 25 & 2.0 & 3.5 & 4.0 & 6.0 & 3.0 \\
\hline 280 & 20 & 20 & 25 & 20 & 25 & as & 3.5 & 3.5 & 5.5 & 3.0 \\
\hline 300 & 20 & 1.5 & 20 & 1.5 & 25 & & 3.0 & 3.0 & 45 & 3.0 \\
\hline 320 & 20 & 1.0 & 1.5 & 1.5 & 20 & & 25 & 25 & 35 & 30 \\
\hline 340 & 15 & & 15 & 1.0 & 15 & & 20 & 25 & 3.5 & 3.0 \\
\hline 360 & 1.0 & & 1.5 & 2.0 & 1.5 & & 20 & 20 & 3.0 & 3.5 \\
\hline 380 & 1.0 & & 1.0 & 0.5 & 1.5 & & 20 & 1.5 & 3.0 & 3.0 \\
\hline 400 & 1.0 & & & 0.5 & 1.5 & & 20 & 1.5 & 25 & $\mathbf{3 , 0}$ \\
\hline 420 & 1.0 & & & 0.5 & 1.0 & & 1.5 & 15 & & 3.0 \\
\hline 440 & 0.5 & & & 0.5 & & & 1.5 & 1.5 & & 25 \\
\hline 460 & 0.5 & & & & & & 1.5 & 1.0 & & 20 \\
\hline 490 & as & & & & & & 15 & 1.0 & & 20 \\
\hline 500 & a.5 & & & & & & 1.5 & 1.0 & & 20 \\
\hline 520 & & & & & & & 1.0 & 1.0 & & 1.5 \\
\hline 540 & & & & & & & & 0.5 & & \\
\hline
\end{tabular}




\begin{tabular}{|c|c|c|c|c|c|c|c|c|c|c|}
\hline \multicolumn{11}{|c|}{ HIDROGRARAS UTIIZADAS PARA SIMULACAO MAR/83 - ABR/83 } \\
\hline HIDROGS $>$ & 31 & 32 & 33 & 34 & 35 & 36 & 37 & 38 & 39 & 40 \\
\hline DLA $\rightarrow$ & 19MAR & $20 \mathrm{MAR}$ & ZMAR & 23MAR & 2ANARR & SABR & GABR & GABR & ISABR & IGABR \\
\hline HORA $\rightarrow$ & 16 & 1450 & 1530 & $\begin{array}{r}15 \\
\end{array}$ & $15: 10$ & 1850 & 320 & 11 & 17 & $21: 30$ \\
\hline $\begin{array}{l}\text { TEMPO } \\
\text { (min) }\end{array}$ & \multicolumn{10}{|c|}{$\begin{array}{l}\text { ALTURAS DE LAMINA D AGUA } \\
\left(\cos ^{*} 2\right)\end{array}$} \\
\hline 0 & 0 & 0 & 0 & 0 & 0 & 0 & 0 & 0 & 0 & 0 \\
\hline 20 & 0 & 0 & 0 & 0 & 0 & 0 & 0 & 0 & 0 & 0 \\
\hline 40 & 0 & 0 & 0 & 0 & 0 & 0 & 0 & 1.0 & as & 0 \\
\hline 60 & 0 & 0 & as & 0 & 0 & 0 & 15 & 20 & 1.0 & 0 \\
\hline 80 & 0 & 0 & 0.5 & 0 & 0 & 0 & 6.5 & 25 & 1.0 & 0 \\
\hline 100 & 1.5 & 0 & 0.5 & 0 & 0.5 & 17.5 & 8.5 & 25 & 1.0 & 0 \\
\hline 120 & 3.0 & 0 & 1.0 & 0 & 0.5 & 160 & 9.5 & 13.0 & 1.0 & 0 \\
\hline 140 & 5.0 & 0 & 1.0 & 0 & 1.0 & 12.5 & 11.0 & 285 & 1.0 & 0 \\
\hline 160 & 5.5 & 0 & 1.0 & 0 & 1.0 & 15.0 & 125 & 37.5 & 1.0 & 0 \\
\hline 180 & 5.5 & 0 & 1.0 & 1.0 & 1.5 & 11.5 & 17.0 & 35.0 & 1.0 & as \\
\hline 200 & 5.0 & as & 1.0 & 45 & 15 & 100 & 17.5 & 28.0 & 0.5 & 1.0 \\
\hline 220 & 40 & a.s & 1.0 & 5.5 & 1.5 & 80 & 160 & 25.0 & & 2.0 \\
\hline 240 & 4.0 & 1.0 & 1.0 & 8.5 & 20 & 7.0 & 125 & 22.0 & & 1.5 \\
\hline 260 & 25 & 1.0 & 1.0 & 8.0 & 1.0 & 6.0 & 10.0 & 190 & & 20 \\
\hline 280 & 2.5 & 1.0 & 10 & 80 & 1.0 & 60 & 7.0 & 180 & & 20 \\
\hline 300 & 2.5 & 1.0 & 1.0 & 80 & 05 & 5.0 & 2.0 & 160 & & 20 \\
\hline 320 & 25 & 1.0 & 10 & 9.5 & as & 40 & 60 & 130 & & 20 \\
\hline 340 & 30 & 1.0 & 10 & $\mathbf{2 0}$ & as & 35 & 5.0 & 100 & & 20 \\
\hline 360 & 40 & 1.0 & 1.0 & 37.5 & & 3.0 & 40 & 8.0 & & 20 \\
\hline 300 & 4.5 & 0.5 & 1.0 & 440 & & 3.0 & 3.5 & 7.0 & & 20 \\
\hline 400 & 4.5 & & 1.0 & 38.5 & & 25 & 30 & 5.5 & & 20 \\
\hline 420 & 4.5 & & as & 325 & & 20 & 25 & 5.0 & & 20 \\
\hline 440 & 5.0 & & & 325 & & 20 & 20 & 3.5 & & 20 \\
\hline 460 & 5.0 & & & 300 & & 1.5 & & 25 & & 20 \\
\hline 400 & 5.0 & & & 25.0 & & & & 20 & & 20 \\
\hline 500 & 40 & & & 20.0 & & & & 20 & & 20 \\
\hline 520 & 3.5 & & & 160 & & & & 20 & & 20 \\
\hline 540 & 3.5 & & & 130 & & & & 20 & & 20 \\
\hline 560 & 3.0 & & & 11.0 & & & & 1.5 & & 20 \\
\hline 580 & 25 & & & 105 & & & & 0 & & 20 \\
\hline 600 & 25 & & & 105 & & & & & & 1.5 \\
\hline$\infty$ & 25 & & & 105 & & & & & & 15 \\
\hline 640 & 20 & & & 120 & & & & & & 1.5 \\
\hline 660 & 20 & & & 11.0 & & & & & & 1.5 \\
\hline 680 & 20 & & & 10.0 & & & & & & 10 \\
\hline 700 & & & & 9.0 & & & & & & 0 \\
\hline 20 & & & & 7.5 & & & & & & \\
\hline 740 & & & & 60 & & & & & & \\
\hline 760 & & & & 5.5 & & & & & & \\
\hline 700 & & & & 0 & & & & & & \\
\hline 800 & & & & 0 & & & & & & \\
\hline 820 & & & & 1.0 & & & & & & \\
\hline 840 & & & & 0.0 & & & & & & \\
\hline
\end{tabular}




\begin{tabular}{|c|c|c|c|c|c|c|c|c|c|c|}
\hline \multicolumn{11}{|c|}{ FIDROGRAPAS UTILIZADAS PARA SIMUL ACAO ABR/B3 - JUN/83 } \\
\hline HIDROGS $>$ & 41 & $\$ 2$ & 43 & 44 & 45 & 46 & 47 & 48 & 49 & 50 \\
\hline $\mathrm{DLA} \rightarrow$ & 20ABR & 21ABR & 24ABR & $26 A B R$ & 3MAI & $20 \mathrm{MAI}$ & $28 \mathrm{MAI}$ & $302 \mathrm{AII}$ & 3UN & 4UN \\
\hline HORA $->$ & 20 & 1630 & $15: 30$ & $13: 50$ & $\therefore 1$ & 5 & 1950 & 2220 & 15 & $2: 50$ \\
\hline $\begin{array}{l}\text { TEMPO } \\
\text { (min) }\end{array}$ & & & & $\begin{array}{l}\text { ALTUR } \\
(\mathrm{cm})^{*}\end{array}$ & $\overline{\mathrm{DELA}}$ & $\overline{\text { INA D': }}$ & JUA & & & \\
\hline 0 & 0 & 0 & 0 & $\therefore 0$ & o & $\overline{0}$ & $\mathbf{0}$ & 0 & o & 0 \\
\hline 20 & 0 & 0 & as & 0 & 0 & 0 & 0 & 0 & 0 & 1.5 \\
\hline 40 & 0 & 0 & 5.5 & 0 & 0 & as & 5.0 & as & 0 & 20 \\
\hline 60 & 1.5 & 3.0 & 5.0 & as & 0 & 0.5 & 7.0 & 0.5 & 0 & 20 \\
\hline 80 & 20 & 40 & 4.5 & 1.5 & 0.5 & 1.0 & 6.5 & 1.0 & 0 & 20 \\
\hline 100 & 1.5 & 5.0 & 5.0 & 20 & 1.0 & 1.0 & 7.0 & 1.0 & 0 & 20 \\
\hline 120 & 20 & 6.0 & 4.0 & 20 & 10 & 1.0 & 7.0 & 1.0 & 0 & 25 \\
\hline 140 & 20 & 7.0 & 3.0 & 20 & 20 & 1.0 & 7.5 & 1.0 & 0.5 & 20 \\
\hline 160 & 20 & 7.0 & 25 & 20 & 25 & 1.0 & 7.5 & 1.0 & 1.5 & 1.5 \\
\hline 180 & 15 & 7.0 & 20 & 15 & 3.0 & 20 & 7.0 & 1.0 & 20 & 1.0 \\
\hline 200 & 2.0 & 7.0 & 20 & 15 & 3.0 & 1.0 & 60 & 1.5 & 30 & 1.0 \\
\hline 220 & & 80 & 1.5 & 1.0 & 3.5 & 1.0 & 5.0 & 1.5 & 3.5 & as \\
\hline 240 & & 8.5 & & 1.0 & 3.5 & 1.0 & 4.0 & 25 & 45 & 0.0 \\
\hline 200 & & 85 & & 1.0 & 40 & 1.0 & 30 & 30 & 45 & \\
\hline 280 & & 8.5 & & 0.5 & 40 & 1.0 & 3.0 & 4.5 & 4.5 & • \\
\hline 300 & & 85 & & & 4.0 & 0.5 & 25 & $\mathbf{7 . 0}$ & 40 & \\
\hline 320 & & 125 & & & 40 & & 25 & 100 & 35 & \\
\hline 340 & & 25.0 & & & 40 & & 20 & 140 & 35 & \\
\hline 360 & & 19.0 & & & 4.0 & & & 14.5 & 3.0 & \\
\hline 380 & & 17.5 & & & 40 & & & 14.5 & 3.0 & \\
\hline 400 & & 15.0 & & & 40 & & & 13.5 & 30 & \\
\hline 420 & & 13.0 & & & 40 & & & 125 & 25 & \\
\hline 440 & & 11.0 & & & 4.0 & & & 125 & 25 & \\
\hline 460 & & 100 & & & 35 & & & 120 & 20 & \\
\hline 480 & & 8.0 & & & 3.0 & & & 11.0 & & \\
\hline 500 & & 7.0 & & & 3.0 & & & 10.0 & & \\
\hline 520 & & 60 & & & 3.0 & & & 90 & & \\
\hline 540 & & 5.5 & & & 25 & & & 7.5 & & \\
\hline 360 & & 50 & & & 20 & & & 7.0 & & \\
\hline 580 & & 5.0 & & & 0 & & & 60 & & \\
\hline 600 & & 45 & & & & & & 60 & & \\
\hline 620 & & 0 & & & & & & 5.5 & & \\
\hline 640 & & & & & & & & 3.0 & & \\
\hline 660 & & & & & & & & 20 & & \\
\hline 680 & & & & & & & & 0.5 & & \\
\hline $\mathbf{2 0 0}$ & & & & & & & & 0 & & \\
\hline
\end{tabular}




\begin{tabular}{|c|c|c|c|c|c|c|c|c|c|c|}
\hline \multicolumn{11}{|c|}{ HIDROGRAPAS UTLUZADAS PARA SIMULACAO JUN/83 - SET/83 } \\
\hline HIDROGS $\rightarrow$ & 51 & 52 & 53 & $\therefore 54$ & 55 & 56 & 57 & 58 & 59 & 60 \\
\hline DLA $\rightarrow$ & QTUN & 28TUN & 18UL & $2 A G O$ & 4AGO & 1GSET & 18SET & ISSET & 22SET & 23SET \\
\hline HORA $\rightarrow$ & 5.30 & $15: 50$ & $5 \times 50$ & 2350 & 740 & 2330 & 1540 & 1420 & 3355 & $3 \times 30$ \\
\hline $\begin{array}{l}\text { Tramo } \\
\text { (min) }\end{array}$ & \multicolumn{10}{|c|}{$\begin{array}{l}\text { AlTURAS DE LARMA DAOUA } \\
(\mathrm{cm} / 2)\end{array}$} \\
\hline 0 & 0 & 0 & 0 & 0 & 0 & 0 & 0 & 0 & 0 & 0 \\
\hline 203 & 0 & 0 & 0 & 0 & 0 & os & 60 & 0 & os & 0 \\
\hline 40 & 2.5 & 7.5 & 1.0 & 10 & 0 & 20 & 125 & 0 & 0.5 & 0 \\
\hline 60 & 145 & 90 & 10 & 1.0 & 0 & 5.5 & 110 & 0 & a.s & 0 \\
\hline$\infty$ & 25.5 & 12.5 & 1.0 & 1.5 & 0 & 5.5 & 160 & as & 4.5 & 0.5 \\
\hline 100 & 23.0 & 140 & 1.5 & 20 & 0 & 6.0 & 13.0 & 0.5 & 6.0 & 0.5 \\
\hline 120 & 24.0 & 11.5 & 1.5 & 20 & 0 & 5.5 & 15.0 & 1.0 & 6.5 & 1.0 \\
\hline 140 & 23.5 & 11.0 & 1.5 & 20 & as & 4.0 & 13.5 & 1.0 & 35 & 1.5 \\
\hline 160 & 21.5 & 9.5 & 1.5 & 20 & 1.0 & 3.5 & 125 & 1.0 & 105 & 20 \\
\hline 180 & 220 & 7.5 & 15 & 20 & 1.0 & 40 & 10.5 & 1.5 & 11.0 & 20 \\
\hline 200 & 130 & 7.0 & 15 & 20 & 10 & 40 & 65 & 15 & 125 & 20 \\
\hline 220 & 23.0 & 6.0 & 1.5 & 20 & 1.0 & 3.5 & 8.0 & 1.5 & 23.0 & 20 \\
\hline 240 & 11.5 & 5.5 & 1.5 & 20 & 1.0 & 3.5 & 7.5 & 2.5 & 11.5 & 1.5 \\
\hline 260 & 10.5 & 5.0 & 1.5 & 20 & 20 & 3.0 & 9.0 & 15 & 11.0 & 1.5 \\
\hline 280 & 9.5 & 40 & 15 & 20 & 1.5 & 25 & 5.5 & 1.0 & 100. & 15 \\
\hline 300 & 80 & 35 & 1.5 & 20 & 1.5 & 20 & 5.0 & 1.0 & 85 & 1.0 \\
\hline 320 & 7.5 & 30 & 1.5 & 20 & 1.5 & 1.5 & 4.0 & 1.0 & 7.5 & 1.0 \\
\hline 340 & 60 & 25 & 20 & 20 & 15 & 10 & 35 & 120 & 7.0 & as \\
\hline 360 & 5.5 & 20 & 20 & 20 & 2.5 & as & & 1.0 & 60 & a.s \\
\hline 380 & 5.0 & & 25 & 20 & 1.5 & & & 1.0 & 5.0 & 0.5 \\
\hline 400 & 4.5 & & 25 & 20 & 15 & & & 1.0 & 4.5 & \\
\hline 420 & 3.5 & & 25 & 1.5 & 1.0 & & & 3.0 & 40 & \\
\hline 440 & 3.5 & & 25 & 1.5 & 1.0 & & & 1.0 & 3.5 & \\
\hline 460 & 25 & & 25 & 1.0 & 1.0 & & & 1.0 & 30 & \\
\hline 480 & 15 & & 20 & 10 & 1.0 & & & 10 & 30 & \\
\hline 500 & 0.0 & & 20 & 1.0 & 1.0 & & & 1.0 & 20 & \\
\hline 520 & & & 20 & as & 1.0 & & & 1.0 & & \\
\hline 540 & & & 20 & & 1.0 & & & 1.0 & & \\
\hline 560 & & & 15 & & 1.0 & & & 1.0 & & \\
\hline 580 & & & & & as & & & 1.0 & & \\
\hline 600 & & & & & & & & 1.0 & & \\
\hline 620 & & & & & & & & 1.0 & & \\
\hline 640 & & & & & & & & 1.0 & & \\
\hline 660 & & & & & & & & 0.5 & & \\
\hline
\end{tabular}




\begin{tabular}{|c|c|c|c|c|c|c|c|c|c|c|}
\hline \multicolumn{11}{|c|}{ HIDROGRAPAS UTHURADAS PARA SIMULACAO SET/33 - NOV/O3 } \\
\hline HIDROGS $>$ & 61 & 62 & $\mathbf{6 3}$ & 64 & 65 & 66 & 67 & 68 & 69 & $\boldsymbol{7 0}$ \\
\hline$D I A->$ & 23SET & 180UT & $220 \mathrm{TT}$ & $2 \mathrm{NOV}$ & 3NOV: & 4NOV & $19 \mathrm{NOV}$ & $20 \mathrm{NOV}$ & $22 \mathrm{NOV}$ & 29NOV \\
\hline$H O R A \rightarrow$ & $16 \times 40$ & $21: 20$ & 910 & 1750 & $15: 10$ & 1430 & 23.15 & $16 \times 10$ & 4 & $15 \times 30$ \\
\hline $\begin{array}{l}\text { TBMPO } \\
\text { (min) }\end{array}$ & \multicolumn{10}{|c|}{$\begin{array}{l}\text { ALTURAS DELAMINA D'AGUA } \\
\left(\mathrm{cm}^{*} 2\right)\end{array}$} \\
\hline 0 & 0 & 0 & 0 & 0 & 0 & 9 & 0 & 0 & 0 & 0 \\
\hline 20 & 0 & 0 & 0 & 0 & 0 & 0 & 3.5 & 1.5 & 1.5 & 0 \\
\hline 40 & 25 & 0 & 0 & 0 & 25 & 20 & 60 & 1.5 & 1.0 & 0.5 \\
\hline 60 & 40 & 0 & 10 & as & 45 & 20 & 5.0 & 25 & 15 & as \\
\hline 80 & 40 & 1.0 & 1.0 & 1.0 & 5.0 & 25 & 5.0 & 25 & 1.5 & 1.0 \\
\hline 100 & 4.5 & 20 & 1.0 & 2.5 & 4.0 & 20 & 6.0 & 3.0 & 15 & 1.0 \\
\hline 120 & 5.5 & 2.5 & 1.5 & 1.5 & 4.5 & 20 & 4.0 & 3.0 & 1.5 & 1.0 \\
\hline 140 & 5.5 & 3.5 & 1.5 & 1.5 & 40 & 20 & 30 & 2.5 & 1.5 & 1.0 \\
\hline 160 & 4.5 & 4.0 & 1.5 & 20 & 4.5 & 20 & 25 & 25 & 1.0 & 1.0 \\
\hline 180 & 4.0 & 45 & 15 & 20 & 60 & 20 & 20 & 20 & 1.0 & 1.0 \\
\hline 200 & 40 & 5.0 & 15 & 20 & 65 & 20 & 20 & 20 & 1.0 & 10 \\
\hline 220 & 3.5 & 4.5 & 1.5 & 20 & 5.0 & 20 & 1.5 & 20 & 0.5 & 1.0 \\
\hline 240 & 3.0 & 40 & 1.5 & 20 & 4.5 & 20 & 0 & 15 & & 1.0 \\
\hline 260 & 25 & 3.5 & 1.5 & 20 & 45 & 20 & & & & a.5 \\
\hline 280 & 20 & 30 & 1.0 & 1.5 & 40 & 20 & & & & \\
\hline 300 & 20 & 25 & 00 & 1.0 & 30 & 20 & & & & \\
\hline 320 & 1.5 & 20 & & & 25 & 20 & & & & \\
\hline 340 & 0 & 15 & & & & 15 & & & & \\
\hline 360 & & 2.5 & & & & 25 & & & & \\
\hline 300 & & 1.0 & & & & 1.5 & & & & \\
\hline 400 & & & & & & 1.0 & & & & \\
\hline 420 & & & & & & a. & & & & \\
\hline
\end{tabular}




\begin{tabular}{|c|c|c|c|c|c|c|c|c|c|c|c|c|}
\hline \multicolumn{13}{|c|}{ HIDROGRAFAS UTIIZADAS PARA SIMULACAODEZ/83 "OUT-NOV/82 } \\
\hline HIDROG & $n$ & 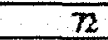 & 73 & 74 & $\therefore 75$ & 76 & $\pi$ & 78 & 79 & 80 & 81 & 82 \\
\hline DIA $->$ & IDEZ & IODEZ & 16DEZ & ITDEZ & $18 \mathrm{DEZ}$ & 19DEZ & 2000Z2 & 25DEZ & 25DEZ & $290 \mathrm{UT}$ & $290 U T$ & $14 \mathrm{NOV}$ \\
\hline HORA-> & $15: 20$ & $15: 50$ & 14 & 17335 & 1930 & 15 & 19 & 2410 & 750 & 5 & $13: 30$ & 950 \\
\hline $\begin{array}{c}\text { TEMPO } \\
\text { (min) }\end{array}$ & & & & $\begin{array}{l}\text { ALTUR } \\
1 \mathrm{~cm}\end{array}$ & SDELA & INA D'A & GUA & & & & & \\
\hline 0 & 0 & 0 & 0 & 0 & 0 & 0 & 0 & 0 & 0 & 0 & 0 & 0 \\
\hline 20 & a.s & as & 0 & 1.5 & 0 & 0.5 & 25 & 0 & 0 & 0 & 0 & 0 \\
\hline 40 & 1.0 & 0.5 & as & 30 & 1.0 & 0.5 & 7.5 & 3.0 & 7.0 & 0.5 & 0 & 0 \\
\hline 60 & 3.5 & 1.5 & 30 & 5.0 & 25 & 1.0 & 21.5 & 15.0 & 7.5 & 1.0 & 0 & 30 \\
\hline 80 & 7.0 & 30 & 45 & 40 & 45 & 30 & 325 & 210 & 100 & 50 & 0 & 5.0 \\
\hline 100 & 13.5 & 60 & 7.0 & 30 & 5.0 & 200 & 320 & 19.5 & 9.0 & 11.0 & 0 & 60 \\
\hline 120 & 13.0 & 6.0 & 5.0 & 3.0 & 5.0 & 29.0 & 22.5 & 14.0 & 7.5 & 22.0 & 0 & 7.0 \\
\hline 140 & 7.0 & 4.5 & 4.0 & 3.0 & 6.0 & 360 & 16.5 & 120 & 6.0 & 11.5 & 0 & 6.5 \\
\hline 160 & 5.0 & 4.0 & 30 & 25 & 5.0 & 25.0 & 130 & 100 & 50 & 105 & 4.0 & 5.5 \\
\hline 180 & 3.5 & 30 & 25 & 20 & 40 & 19.0 & 105 & 80 & 40 & 80 & 6.5 & 5.0 \\
\hline 200 & 3.0 & 25 & 20 & & 3.5 & 14.0 & 8.5 & 7.0 & 30 & 7.0 & 6.0 & 60 \\
\hline 2230 & 25 & 25 & 20 & & 30 & 11.5 & 7.0 & 6.0 & 3.0 & 60 & 9.5 & 7.0 \\
\hline 240 & 20 & 20 & 20 & & 25 & 9.5 & 60 & 50 & 3.0 & 5.0 & 75 & 8.0 \\
\hline 260 & 15 & 20 & 1.5 & & & 80 & 8.0 & 4.5 & 3.0 & 4.0 & 7.0 & 9.0 \\
\hline 280 & 1.5 & 15 & & & & 7.0 & 5.5 & 4.0 & 20 & 3.5 & 7.5 & 10.5 \\
\hline 300 & 10 & 1.5 & & & & 60 & 45 & 35 & & 30 & 7.5 & 130 \\
\hline 320 & & 1.0 & & & & 5.5 & 40 & 3.0 & & 30 & 80 & 17.0 \\
\hline 340 & & & & & & 50 & 35 & & & 25 & 11.5 & 200 \\
\hline 360 & & & & & & 45 & 30 & & & 25 & 160 & 140 \\
\hline 380 & & & & & & 40 & 25 & & & 20 & 180 & 11.5 \\
\hline 400 & & & & & & 3.5 & 20 & & & 20 & 180 & 100 \\
\hline 420 & & & & & & 35 & & & & 20 & 14.5 & 9.0 \\
\hline 40 & & & & & & 30 & & & & 1.5 & 120 & 80 \\
\hline 460 & & & & & & & & & & 1.5 & 100 & 8.0 \\
\hline 480 & & & & & & & & & & 1.5 & 8.5 & 7.0 \\
\hline 500 & & & & & & & & & & 15 & 7.0 & 60 \\
\hline 520 & & & & & & & & & & 20 & 6.0 & 5.0 \\
\hline 540 & & & & & & & & & & & 5.0 & 4.0 \\
\hline 560 & & & & & & & & & & & 4.0 & 35 \\
\hline 580 & & & & & & & & & & & 35 & 3.0 \\
\hline 600 & & & & & & & & & & & 30 & 30 \\
\hline 000 & & & & & & & & & & & 25 & 25 \\
\hline 640 & & & & & & & & & & & 20 & \\
\hline 660 & & & & & & & & & & & 1.5 & \\
\hline 680 & & & & & & & & & & & 1.5 & \\
\hline 700 & & & & & & & & & & & 10 & \\
\hline
\end{tabular}




\section{APENDICE 3}

VALORES DE "Ra" 
VALORES DE MÉJIA DESVIO PADRÄ) E DISTÁNCLA RA DOS DESVIOS ABSOLUTOS DAS SMULACÓES DE IIIRRÓGRAFAS (CLASSE DE VOLUME 70cm)

\begin{tabular}{|c|c|c|c|c|c|c|c|c|c|}
\hline \multirow{2}{*}{$\begin{array}{l}\text { No } \\
\text { VÃÃ }\end{array}$} & \multicolumn{2}{|c|}{$\begin{array}{l}\text { MEDLA } \\
\mathrm{Xm}\end{array}$} & \multicolumn{2}{|c|}{$\begin{array}{c}\text { DESVIO PADRAD } \\
\mathrm{s}\end{array}$} & \multicolumn{2}{|c|}{$\begin{array}{c}\text { DISTÁNCLA } \\
\text { RA }\end{array}$} & \multirow[t]{2}{*}{$\mathrm{DF}$} & \multicolumn{2}{|c|}{ OBSERVAĈ́,O } \\
\hline & MSHJ & MSHA & MSHI & MSHA & MSHJ & MSHA & & MSHI & MSHA \\
\hline 44 & 1.58 & 0.25 & 2.62 & 0.24 & 3.06 & 0.35 & 2.71 & PICO NO FWM & $\mathbb{M P}$ \\
\hline 46 & 0.57 & 0.31 & 0.56 & 0.25 & 0.80 & 0.40 & 0.40 & $S /$ RESPOSTA & \\
\hline 33 & 0.71 & 0.31 & 0.44 & 0.34 & 0.84 & 0.46 & 0.38 & PICO NO FIM & \\
\hline 70 & 0.70 & 0.35 & 0.41 & 0.32 & 0.81 & 0.47 & 0.34 & $S$ / RESPOSTA & \\
\hline 64 & 0.89 & 0.36 & 0.88 & 0.36 & 1.25 & 0.51 & 0.74 & S/ RESPOSTA & \\
\hline 39 & 0.73 & 0.39 & 0.41 & 0.41 & 0.83 & 0.57 & 0.27 & S/ RESPOSTA & \\
\hline 63 & 1.27 & 0.42 & 1.13 & 0.39 & 1.70 & 0.58 & 1.12 & PICO NO FM & \\
\hline 15 & 0.73 & 0.46 & 0.55 & 0.41 & 0.91 & 0.62 & 0.29 & & MP \\
\hline 18 & 2.25 & 0.37 & 2.19 & 0.53 & 3.14 & 0.65 & 2.50 & PICO NO FM & $\mathbb{M P}$ \\
\hline 69 & 0.90 & 0.48 & 0.65 & 0.44 & 1.11 & 0.65 & 0.46 & PICO NO FIM & \\
\hline 55 & 0.70 & 0.46 & 0.53 & 0.48 & 0.87 & 0.67 & 0.21 & PICO INTCIO & \\
\hline 21 & 0.90 & 0.49 & 0.64 & 0.48 & 1.10 & 0.68 & 0.42 & PICO NO FM & \\
\hline 60 & 0.61 & 0.53 & 0.47 & 0.44 & 0.77 & 0.69 & 0.09 & & \\
\hline 16 & 2.01 & 0.50 & 1.68 & 0.51 & 2.62 & 0.71 & 1.91 & PICO NO FM & $\mathrm{MP}$ \\
\hline 58 & 0.45 & 0.59 & 0.28 & 0.42 & 0.53 & 0.73 & -0.19 & MEIHOR & $\mathbf{M P}$ \\
\hline 35 & 1.23 & 0.50 & 3.11 & 0.55 & 3.35 & 0.74 & 2.61 & & \\
\hline 20 & 1.43 & 0.60 & 0.99 & 0.44 & 1.74 & 0.74 & 1.00 & PICO NO FTM & MP \\
\hline 41 & 1.45 & 0.55 & 1.84 & 0.58 & 2.34 & 0.80 & 1.54 & PICO NO FIM & \\
\hline 54 & 1.49 & 0.64 & 0.70 & 0.48 & 1.64 & 0.80 & 0.85 & $S$ / RESPOSTA & \\
\hline 25 & 1.36 & 0.62 & 1.04 & 0.51 & 1.72 & 0.80 & 0.91 & PICO NO FIM & MP \\
\hline 40 & 1.13 & 0.62 & 0.95 & 0.58 & 1.48 & 0.85 & 0.63 & PICO NO FLM & \\
\hline 26 & 1.46 & 0.66 & 1.24 & 0.54 & 1.91 & 0.85 & 1.06 & & \\
\hline 68 & 1.65 & 0.73 & 0.95 & 0.52 & 1.90 & 0.89 & 1.01 & PICO NO FTM & $\mathrm{MP}$ \\
\hline 9 & 1.63 & 0.73 & 0.61 & 0.61 & 1.74 & 0.95 & 0.79 & PICO NICIO & $\mathbb{M P}$ \\
\hline 17 & 2.46 & 0.63 & 2.47 & 0.77 & 3.49 & 0.99 & 2.49 & INYERSAO & \\
\hline 50 & 1.10 & 0.59 & 1.46 & 0.83 & 1.83 & 1.01 & 0.82 & $S$ / RESPOSTA & \\
\hline 66 & 1.73 & 0.86 & 1.92 & 0.57 & 2.59 & 1.03 & 1.56 & PICO NO FLM & \\
\hline 75 & 3.69 & 0.72 & 3.26 & 0.75 & 4.93 & 1.04 & 3.88 & PICO NO FT & MP \\
\hline 32 & 0.79 & 0.69 & 2.30 & 0.85 & 2.44 & 1.09 & 1.34 & PICO INICIO & \\
\hline 8 & 1.51 & 0.89 & 0.64 & 0.64 & 1.65 & 1.10 & 0.54 & PICO NO FI & \\
\hline 14 & 4.84 & 0.85 & 12.27 & 0.75 & 13.19 & 1.13 & 12.06 & PICO INICIO & \\
\hline 24 & 1.51 & 0.94 & 1.26 & 0.67 & 1.97 & 1.16 & 0.81 & PICO NO FTM & \\
\hline 30 & 2.89 & 0.88 & 3.26 & 0.81 & 4.35 & 1.19 & 3.16 & PICO ENTCIO & \\
\hline 11 & 2.01 & 0.88 & 1.91 & 0.84 & 2.78 & 1.21 & 1.56 & S/ RESPOSTA & $\mathbb{M P}$ \\
\hline 65 & 4.20 & 1.06 & 4.29 & 0.88 & 6.00 & 1.38 & 4.63 & PICO NO FL & $\mathrm{MP}$ \\
\hline
\end{tabular}

OBSERVACAO : MP $=$ ESCOLHIDA P/ MPRESSAO 
VALORES DE MÉDLA DESVO PADRÃO E DISTÂNCIA RA DOS DESVOS ABSOLUTOS DAS SIMULACOES DE IIDRÓGRAFAS (CLASSE DE WOLUME 70cm) CONT.

\begin{tabular}{|c|c|c|c|c|c|c|c|c|c|}
\hline \multirow{2}{*}{ 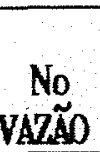 } & \multicolumn{2}{|c|}{$\begin{array}{l}\text { MDIA } \\
\text { Xm }\end{array}$} & \multicolumn{2}{|c|}{$\begin{array}{c}\text { DESVO PADRÁO } \\
\mathrm{s}\end{array}$} & \multicolumn{2}{|c|}{$\begin{array}{l}\text { DISTANCIA } \\
\text { RA }\end{array}$} & \multirow[t]{2}{*}{ DIF } & \multicolumn{2}{|c|}{ OASERVACÃO } \\
\hline & NSHI & MSHA & MSHJ & MSHA & MSHJ & MSHA & & MSHJ & MSHA \\
\hline 28 & 1.96 & 1.21 & 1.20 & 0.82 & 2.30 & 1.46 & 0.84 & S/ RESPOSTA & \\
\hline 61 & 4.33 & 1.14 & 5.40 & 1.00 & 6.92 & 1.51 & 5.41 & PICO NO FM & \\
\hline 43 & 3.53 & 1.14 & 5.08 & 1.06 & 6.19 & 1.55 & 4.63 & PICO ENCIO & \\
\hline 2 & 1.28 & 1.24 & 1.02 & 1.02 & 1.64 & 1.61 & 0.03 & & IMP \\
\hline 62 & 3.11 & 1.20 & 4.15 & 1.11 & 5.18 & 1.63 & 3.55 & PICO NO FTM & \\
\hline 74 & 2.32 & 1.39 & 2.59 & 1.01 & 3.48 & 1.71 & 1.77 & PICO NO FM & \\
\hline 67 & 3.73 & 1.28 & 3.19 & 1.15 & 4.91 & 1.72 & 3.19 & $\mathrm{PICO}$ NO FM & \\
\hline 3 & 1.74 & 1.05 & 1.37 & 1.37 & 2.21 & 1.72 & 0.49 & & MP \\
\hline 7 & 2.11 & 1.12 & 1.31 & 1.31 & 2.48 & 1.72 & 0.75 & PICO NO FW & DMP \\
\hline 56 & 3.36 & 1.20 & 4.66 & 1.38 & 5.74 & 1.83 & 3.91 & PICO NO FM & \\
\hline 73 & 2.34 & 1.37 & 2.16 & 1.44 & 3.19 & 1.99 & 1.20 & PICO NO FIM & \\
\hline 49 & 1.78 & 1.75 & 2.71 & 1.10 & 3.24 & 2.06 & 1.18 & & MP \\
\hline 5 & 5.11 & 1.53 & 1.56 & 1.56 & 5.35 & 2.18 & 3.16 & & \\
\hline 72 & 2.58 & 1.56 & 3.09 & 1.57 & 4.03 & 2.21 & 1.82 & & \\
\hline 71 & 6.00 & 1.73 & 5.00 & 1.44 & 7.80 & 2.25 & 5.55 & & MPP \\
\hline 6 & 1.98 & 1.85 & 1.60 & 1.60 & 2.55 & 2.45 & 0.10 & & \\
\hline 53 & 4.12 & 1.52 & 14.07 & 1.95 & 14.66 & 2.47 & 12.19 & PICO INCIO & $\mathrm{IMP}$ \\
\hline 4 & 5.18 & 2.03 & 1.74 & 1.74 & 5.47 & 2.68 & 2.79 & & $\mathbb{M P}$ \\
\hline
\end{tabular}


VALORES DE MÉDLL DESVIO PADRÃO E DISTÂNCLA RA DOS DESVIOS ABSOLUTOS DAS SMMULCÓES DE HIIRROGRAFAS (CLASSE DE VOLUME 140cm) CONT.

\begin{tabular}{|c|c|c|c|c|c|c|c|c|c|}
\hline \multirow{2}{*}{$\begin{array}{l}\text { No } \\
\text { VAZAO }\end{array}$} & \multicolumn{2}{|c|}{$\begin{array}{l}\text { MFDIA } \\
\text { Xm }\end{array}$} & \multicolumn{2}{|c|}{$\begin{array}{l}\text { DESVIO PADRÁ } \\
\mathrm{s}\end{array}$} & \multicolumn{2}{|c|}{$\begin{array}{c}\text { DISTANCIA } \\
\text { RA } \\
\end{array}$} & \multirow[t]{2}{*}{$\mathrm{DF}$} & \multicolumn{2}{|c|}{ OBSERVACÃO } \\
\hline & MSH. & MSHA & MSHJ & MSHA & MSHJ & MSHA & & MSHJ & MSHA \\
\hline 22 & 1.45 & 0.47 & 2.16 & 0.60 & 2.60 & 0.76 & 1.84 & PICO NO FMM & LMP \\
\hline 31 & 1.39 & 1.10 & 1.40 & 0.76 & 1.94 & 1.34 & 0.60 & & MP \\
\hline 47 & 6.35 & 1.00 & 5.95 & 1.04 & 8.70 & 1.44 & 7.26 & PICO NO FIM & MPP \\
\hline 1 & 2.78 & 1.11 & 1.02 & 1.02 & 2.96 & 1.51 & 1.45 & & MPP \\
\hline 10 & 5.16 & 1.14 & 0.99 & 0.99 & 5.25 & 1.51 & 3.74 & PICO INICIO & IMP \\
\hline 45 & 1.58 & 1.16 & 1.05 & 1.04 & 1.89 & 1.56 & 0.33 & PICO NO FIM & \\
\hline 19 & $3.7 t^{\circ}$ & 1.19 & 3.04 & 1.19 & 4.82 & 1.68 & 3.14 & & MPP \\
\hline 12 & 3.15 & 1.39 & 2.46 & 1.40 & 3.98 & 1.97 & 2.01 & INYERSAO & $\mathrm{MP}$ \\
\hline 80 & 1.35 & 1.54 & 0.91 & 1.39 & 1.62 & 2.07 & -0.45 & MEIHOR & $\mathbb{M P}$ \\
\hline 79 & 4.4 & 1.77 & 5.58 & 1.91 & 7.14 & 2.60 & 4.53 & PICO NO FM & \\
\hline 52 & 4.0 & 1.77 & 3.62 & 2.00 & 5.41 & 2.67 & 2.74 & & $\mathbb{M P}$ \\
\hline 27 & $2.8 \mathrm{f}$ & 2.44 & 2.27 & 2.06 & 3.65 & 3.20 & 0.45 & S/ RESPOSTA & $\mathrm{MP}$ \\
\hline 78 & $8.0^{\circ}$ & 3.47 & 9.11 & 4.43 & 12.17 & 5.62 & 6.55 & & IMP \\
\hline 23 & 6.0 & 4.06 & 6.78 & 5.12 & 9.09 & 6.53 & 2.56 & PICO NO FM & $\mathrm{MP}$ \\
\hline
\end{tabular}

OASERVACAD : IMP = ESCOLHIDA P/ IMPRESSAO

VALORES DE MFDIA DESVO PADRAD E DISTANCLA RA DOS DESVIOS ABSOLUTOS DAS STMULCOES DE HIDROGRAFAS (CLASSE DE VOLUME $210 \mathrm{~cm}$ ) CONT.

\begin{tabular}{|c|c|c|c|c|c|c|c|c|c|}
\hline \multirow{2}{*}{$\begin{array}{l}\text { No } \\
\text { VAZAO }\end{array}$} & \multicolumn{2}{|c|}{$\begin{array}{l}\text { MEDLA } \\
\mathrm{Xm}\end{array}$} & \multicolumn{2}{|c|}{$\begin{array}{c}\text { DESYO PADRAO } \\
\mathrm{s}\end{array}$} & \multicolumn{2}{|c|}{$\begin{array}{c}\text { DISTANCLA } \\
\text { RA }\end{array}$} & \multirow[t]{2}{*}{ DFF } & \multicolumn{2}{|c|}{ OBSERVACAO } \\
\hline & MSHU & MSHA & MSHI & MSHA & MSHJ & MSHA & & MSHI & MSHA \\
\hline 29 & 5.85 & 1.63 & 4.47 & 2.02 & $7.36^{\circ}$ & 2.60 & 4.77 & & $\mathbb{M P}$ \\
\hline 36 & 2.91 & 2.14 & 5.57 & 2.27 & 6.28 & 3.12 & 3.16 & & MPP \\
\hline 59 & 3.00 & 2.78 & 2.42 & 2.03 & 3.86 & 3.45 & 0.41 & & $\mathrm{MP}$ \\
\hline 37 & 16.88 & 2.69 & 49.34 & 2.40 & 52.15 & 3.60 & 48.54 & PICO INICIO & MP \\
\hline 57 & 4.62 & 3.28 & $\mathbf{3 . 3 7}$ & 2.69 & 5.72 & 4.24 & 1.48 & & MPP \\
\hline 81 & 15.28 & 3.04 & 48.34 & 3.29 & 50.70 & 4.48 & 46.22 & PICO INICIO & IMP \\
\hline 48 & 11.82 & 4.58 & 47.23 & 3.00 & 48.68 & 5.47 & 43.21 & PICO INCIO & $\mathrm{MP}$ \\
\hline
\end{tabular}

OBSERVACAO : MP = ESCOLHIIA P/ MPRESSAD 
VALORES DE MÉELIA DESTO PADRÃO E DISTÂNCIA RA DOS DESVTOS ABSOLUTOS DAS SMUULACÓNS DE HIDRÓGRAFAS (CLASSE DE VOLUME $280 \mathrm{~cm}$ ) CONT.

\begin{tabular}{|c|c|c|c|c|c|c|c|c|c|}
\hline \multirow{2}{*}{$\begin{array}{c}\text { No } \\
\text { VAZAD } \\
\end{array}$} & \multicolumn{2}{|c|}{$\begin{array}{c}\text { MEDIA } \\
\text { Xm } \\
\end{array}$} & \multicolumn{2}{|c|}{ DESVO PADRAOD } & \multicolumn{2}{|c|}{ DISTANCIA } & \multirow[t]{2}{*}{$\mathrm{DF}$} & \multicolumn{2}{|c|}{ OBSERVACANO } \\
\hline & MSHJ & MSHA & MSHJ & MSH: & MSHJ & MSHA & & MSHI & MSHA \\
\hline 82 & 10.90 & 2.75 & 24.42 & 3.22 & 26.75 & 4.23 & 22.51 & PICO INICIO & $\mathrm{IMP}$ \\
\hline 51 & 11.43 & 3.32 & 15.11 & 3.42 & 18.95 & 4.77 & 14.18 & & MP \\
\hline 42 & 15.36 & 2.84 & 34.74 & 3.85 & 37.98 & 4.78 & 33.20 & PICO INICIO & $\mathbb{M P}$ \\
\hline 76 & 6.60 & 4.62 & 7.93 & 6.15 & 10.32 & 7.70 & 2.62 & & MP \\
\hline 77 & 9.07 & 5.29 & 9.39 & 6.65 & 13.05 & 8.50 & 4.55 & PICO NO FWM & IMP \\
\hline
\end{tabular}

OBSERVACAO : IMP = ESCOLHIDA P/ IMPRESSEO

VALORES DE MÉDIA DESVIO PADRÃO E DISTÂNCLA RA DOS DESVOS ABSOLUTOS DAS STMULACOOES DE HIDROGRAFAS (CLASSE DE VOLUME $350 \mathrm{~cm}$ ) CONT.

\begin{tabular}{|c|c|c|c|c|c|c|c|c|c|}
\hline \multirow{2}{*}{$\frac{\text { No }}{\text { VAZAD }}$} & \multicolumn{2}{|c|}{$\begin{array}{l}\text { MEDLA } \\
\mathrm{Xm}\end{array}$} & \multicolumn{2}{|c|}{$\begin{array}{c}\text { DESVO PADRAO } \\
\mathrm{s} \\
\end{array}$} & \multicolumn{2}{|c|}{$\begin{array}{c}\text { DISTANCLA } \\
\text { RA } \\
\end{array}$} & \multirow[t]{2}{*}{ DIF } & \multicolumn{2}{|c|}{ OBSERVACAD } \\
\hline & $\overline{\text { MSHJ }}$ & MSHA & MSEJ & MSHA & MSHJ & MSHA & & MSHJ & MSHA \\
\hline 13 & 29.93 & 4.94 & 113.04 & 4.1 & 116.9 & 6.47 & 110.5 & PICO INICIO & MPP \\
\hline 38 & 30.96 & 4.19 & 111.41 & 5.55 & 115.6 & 6.96 & 108.7 & PICO INICIO & MP \\
\hline
\end{tabular}

OBSERVACAOA : MPP = ESCOLHIDA P/ IMPRESSAO

VALORES DE MÉDLA DESVO PADRÃO E DISTANACIA RADOS DESVIOS ABSOLUTOS DAS SMULACÕES DE HIIBÓGRAFAS (CLASSE DE VOLUME 490cm) CONT.

\begin{tabular}{|c|c|c|c|c|c|}
\hline No & $\begin{array}{l}\text { MEDIA } \\
\mathrm{Xm}\end{array}$ & DESVIO PADRAD & $\begin{array}{c}\text { DISTANCLA } \\
\text { RA }\end{array}$ & DFF & OASERVACÃ \\
\hline VAZAO & MSHJ & MSHJ & MSHA & & MSHJ MSHA \\
\hline 34 & 26.61 & $98.02 \quad 10.83$ & 101.6 & 86.45 & PICO INICIO MP \\
\hline
\end{tabular}




\section{APÊNDICE 4}

ROTINAS DO PASH (1inguagem SAS) 


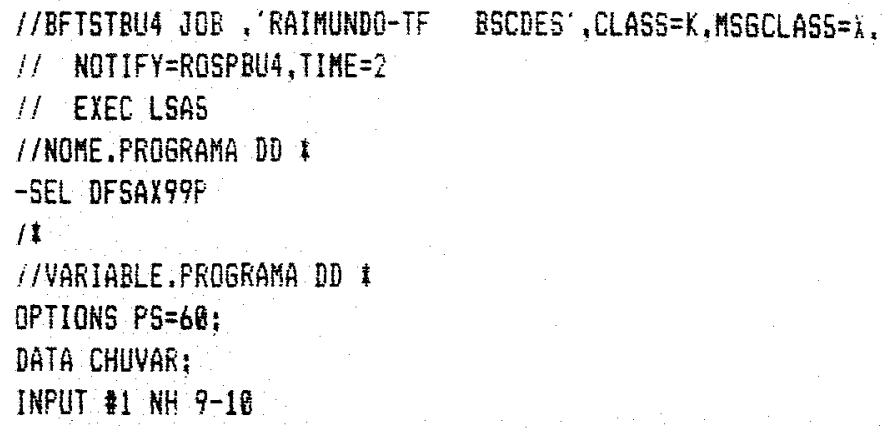

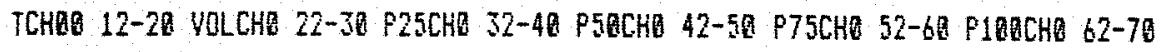
$\$ 2$ NH1 $9-10$

TCHB1 12-2B VOLCH1 22-3A F25CH1 32-40 P50CH1 42-58 f75CH1 52-60 P16ACH1 62-72 *N NH2 $9-19$

TCH92 12-20 VOLCH2 22-30 P25CH2 32-40 P59CH2 42-5a P75CH2 $52-60$ Ple日CH2 62-72 \# $\mathrm{NHZ}$ ? $9-18$

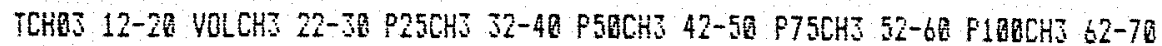
$\$ 5$ NH4 $9-18$

TCHQ4 12-20 VOLCH4 22-30 P25CH4 32-40 P5GCH4 42-56 P75CH4 52-68 P190CH4 62-70 \$6 N45 9-10

TCHOS 12-20 VOLCHS 22-30 P25CHS 32-40 P58CH5 42-50 P75CH5 52-60 F1ARCH5 62-70; DROP NH1 NH2 NH3 NH4 NHS;

CARDS:

\begin{tabular}{|c|c|c|c|c|c|}
\hline 0.000 & 31.000 & 1.833 & 5.600 & 8.883 & 14.667 \\
\hline 0.800 & 0.000 & 0.000 & 8.800 & 0.000 & 0.000 \\
\hline 0.026 & 0.000 & 0.000 & 0.000 & 0.000 & 0.008 \\
\hline 0.000 & 0.003 & 0.060 & 0.000 & 0.000 & 0.000 \\
\hline 0.080 & 0.000 & 0.000 & 0.680 & 0.806 & 0.800 \\
\hline 6.869 & 0.009 & 6.600 & 0.000 & 0.008 & 0.000 \\
\hline 0.000 & 25.500 & 3.421 & 3.657 & 4.454 & 6.690 \\
\hline 0.060 & 0.000 & B.8Bo & 0.000 & 0.680 & 0.082 \\
\hline 0.000 & Q.808 & 0.080 & 0.806 & 0.000 & 0.000 \\
\hline 0.000 & 0.000 & 0.000 & 0.600 & 0.000 & 0.000 \\
\hline 0.005 & 0.800 & 0,090 & Q.6Be & Q.020 & 0.000 \\
\hline Q.8Ag & 0.000 & 0.600 & 0.060 & 0.800 & 0,060 \\
\hline 0.000 & 11.902 & 1.061 & 1.158 & 1.254 & 1.667 \\
\hline 34.183 & 25.510 & 3.421 & 3.657 & 4.454 & 6.000 \\
\hline 0.069 & 0.000 & B. $00 \mathrm{n}$ & $0.8 B D$ & 0.800 & 0.000 \\
\hline 0.060 & 0.030 & 0.000 & 0.000 & 0.806 & 0.060 \\
\hline 0.000 & 0.908 & 0.000 & 0.080 & 8.680 & B. 080 \\
\hline 0.006 & 0.000 & 8.080 & 6.800 & 0.000 & 0.000 \\
\hline . & & $\therefore$ & 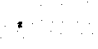 & & \\
\hline ' & - & . & . & . & \\
\hline ' & . & . & , & . & \\
\hline ' & . & . & . & : & \\
\hline$\therefore$ & : & $\therefore$ & $\cdot$ & $\cdot$ & \\
\hline 0.609 & 39.802 & 2.586 & 4.167 & 5.300 & 8.33 \\
\hline (1. .100 & 0.098 & Q.60 & a.6an & 0.89? & 0.000 \\
\hline 6.006 & 0.609 & B. 680 & B.000 & 0.608 & 0.608 \\
\hline
\end{tabular}


MODEL LAMBDA=LF1BOCHA INTCHO LP2SCHY INTSRCHQ/NOINT F;

PROC REG DATA=ALL;

MODEL LLAMBDA=LP1ORCHO INTCHB LP25CHQ INTSECHO/NOINT P;

PROC REG DATA=ALL:

MODEL Y13=P1QRCHO P5QCHO P75CHQ/NOINT $F$;

PROC RES DATA=ALL;

MODEL GAMA=INT75CHS LP25CH3 P2SCH1 LP5BCH1/NOINT FIN

/TPROC STEPHISE DATA=TOTAL;

MODEL NETA LNETA GAMA LGAMA =

TCHDO VOLCHO P25CHO P5OCHO P75CHE P1BACHO

TCHO1 VOLCH1 P25CH1 P5BCH1 P75CH1 PIBBCH1

$\mathrm{TCH}_{2}$ VOLCH2 P25CH2 P5QCH2 P75CH2 P1OaCH2

TCHES VOLCH3 F25CHS P5OCHS F75CHS P1QBCHS

TCHQ4 VOLCH4 P25CH4 P5QCHA P75CH4 P1BACH4

TCHOS VQLCHS P2SCHS PSOCHS P7

LTCHAR LVOLCHA LP2SCHE LPSECHO LF75CHA LPIOACHE

LTCHO1 LVLCH1 LP25CH1 LP5OCH1 LP75CH1 LPIBQCHI

LTCHO2 LVOLCH2 LP25CH2 LP5ECH2 LP75CH2 LF1BOCH2

LTCHO LUDLCHS LP25CHS LPSACHS LP75CHS LP1QQCHS

LICHDA LVOLCH4 LF25CH4 LPSOCH4 LP75CH4 LP10OCH4

LTCHQ5 LYOLCHS LP25CHS LP5BCHS LP75CHS LPIOBCHS

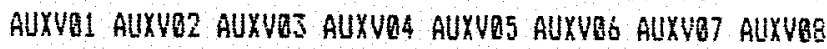

AUXYG9 AUXV1B AUXV11 AUXV12 AUX1 AUK2 AUXS AUR4

Rut?:

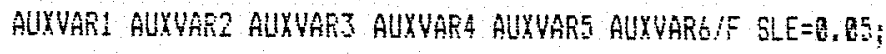

IFFTOC REG DATA=TOTAL;

MODEL VALAC= AUVWOL PIOQCH4 P75CH4 AUXVQ4 /NOINT $F$;

FROC REG DATA=TOTAL:

MONEL VALAC= AUXVGI PlOQCH4 P75CH4 AUXVBS / P;H

IAPROC REG DATA=TOTAL;

MODEL NETA = P25CH4 TCHO1 VOLCHA P5OCHE LVOLCHS VOLCH3/NOINT PII

/AFFOC PLOT DATA= TOTAL:

PLOT Y13*VDLCH1/ UREF $=0$;

PLOT Y13tP25CH1 $/$ UREF $=0$ :

PLOT Y13TFSDCH1/ VREF $=0$;

FLOT Y1JTPIBQCH1/ VREF=e;

FLOT Y13TCHA1/ VREF $=0$ :

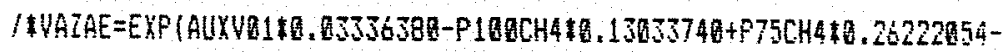

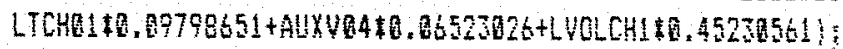

PROC PRINT;

VAR ESILON EESP LAMBDA LESP NETA HESP GAMA GESP WAZAC VALAE: 
RUN:

IFPFOC STEPUISE DATA=ALL;

MODEL Y1 Y2 Y3 Y4 Y5 Y6 Y7 Y8 Y9 Y10 Y11 Y12 Y13 Y14 Y15 Y16

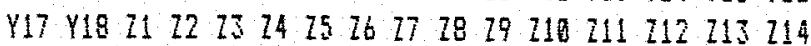
715 Z16 217 Z18 LY1 LY2 LYS LYA LYS LYG LY? LYB LYG LY10 LY11 LY12 LY13 LY14 LYIS LY16 LY17 LYIR= TCHAR VOLCHO P25CHE P5OCHE P75CHE F19BCHE TCHA1 VOLCH1 P25CH1 PSOCH1 P7SCH1 P168CHI TCHO2 VOLCH2 P2SCH2 FSBCH2 P75CH2 P1BGCH2 TCHES VOLCHS F25CHS P5ECH3 P75CH3 P1OBCH3 TCHOS 4 VOLCH4 P25CH4 P5OCH4 P75CH4 P1OOCH4 TCHOS VOLCHS P2SCHS P5OCH5 P75CH5 P18OCHE LTCHRO LYOLCHO LP2SCHE LP5DCHO LP75CHE LPIBQCHO LTCHO1 LUOLCH1 LF25CH1 LP5QCH1 LP7SCH1 LP1OBCHI LTCH22 LVOLCH2 LP25CH2 LP5ACH2 LP75CH2 LP1OBCH2 LTCHQS LVQLCH LP 2ECHS LPSECHS LPTSCHS LFIORCHS LTCHQ4 LUOLCH4 LP25CH4 LPSACH4 LP75CH4 LP16BCH4 LTCHOS LVOLCHE LP2SCHS LPSOCHE LP7SCHS LP1OACHE SiN: $: 1$ IWT2SCHE IWTSBCHO INT7SCHO IMTCHE IF SLE=Q.05;

If FRDC REG DATA=TOTAL;

MODEL VALAC =AUWVO1 PIOACH4 F75CH4 LTCHOL AUXVQA LVOLCHI/ P; MODEL LUAZAC =AUXVOL PIGBCHA P75CH4 LTCHO1 AUXVQ4 LVOLCH1/ $\mathrm{F}$; MODEL VA2D =VOLCH1 LVOLCH1 P5ACH3 P25CH3 LF75CHS INTCHQ/ F; MODEL VAZB =L VOLCHI PSECHZ P25CH3 LP75CH3 INTCHQ/ F: MODEL VAZCUEO=F108CH4 P5OCHA P5QCH1 TCHQ4 P2SCH4 P75CH4 P2SCH1 / P:

MODEL YAZCURO=IP1QRCH4 LPSACH4 LPSBCH1 TCHQ4 LP25CH4 F75CHA LP25CHL / PI\#!

/APROC FLOT DATA = TOTAL;

PLOT VAZACVULCH1/ UREF $=$ :

FLOT VAZACWVLCHZ/ UREF =?

FLOT VAZAC UOLCH $4 /$ VREF $=8$ ?

FLOT VALACWLVOLCH1/ VREF=:;

PLOT LVAZACWOLCHI/ VREF =

PLOT LUAZACUVOLCHZ 2 UREF $=0$;

FLOT LVAZACUVLCH4/ VREF $=0$;

PLDT LVALACUVLCH4/ YREF $=0$;

PLOT LVAIACLLVOLCHI/ VREF = ;

IFPROC UHIVARIATE DATA=VAIAO PLOT NORMAL FREQ;

VAR LVAZAC:

PROC CHART DATA=VAZAO;

VBAR LVAZAC;"!

/IPROC REG DATA=ALL:

MODEL EFSILON=LP35CHA INTCHO PSBCHO INT2SCHQ/NOINT P;

PROC REG DATA=ALL;

MODEL LEPSILON=LP25CHO INTCHE F5OCHO IHT25CHM/NOINT

PROC FEG DATA=ALL: 
FLOT NETALFT5CHA $/$ VREF = R;

PLOT NETA TP75CH5/VREF = :

PLOT NETALPIBECHE/UREF $=$;

PLOT NETATP1QGCH1/VREF $=$ ?;

PLOT NETAIP1GBCH2/UREF $=8$;

PLOT NETALPIBECHS/UREF $=$ ?;

PLOT NETATPIOQCH4/NREF = : ;

PLOT NETATPIBQCHS/VREF $=8 ;$; /

If PROC STEPWISE DATÁ=TOTAL:

MDDEL NETA =

TCHQS VOLCHO P25CHO F5OCHO P75CHO F1OOCHO

TCHB1 VOLCH1 P25CHI P5OCH1 P75CH1 PIOOCH1

TCHO2 VOLCH2 P25CH2 P5aCH2 P75CH2 P100CH2

TCHOS UDLCHS P25CHS P5ECHS P75CH $3100 \mathrm{CHS}$

TCHO4 VOLCH4 P25CH4 P5QCH4 F75CH4 P1QBCH4

TCHOS VOLCHS P25CHS P5BCHS P75CHS PIOACHS

LTCHAO LVOLCHO LP2SCHO LPSACHE LP75CHE LPIGBCHO

LTCHO1 LVOLCH LF25CHI LPSOCH LP75CH1 LPIOOCHI

LTCHQ2 LVOLCH2 LP25CH2 LP5OCH2 LP75CH2 LP100CH2 LTCHOS LVOLCHS LP25CHS LP5OCHS LP75CHS LP1BOCHS LTCHO4 LVOLCH4 LP2SCH4 LP5BCHA LP75CH4 LP1OACH4 LTCHOS LUOLCHS LP25CHS LP5OCHS LP75CHS LP10QCHS INT2SCHO INTSBCHO INT75CHO INTCHO /F SLE=0.05;

RUN:?

ITPROC STEPHISE DATA=ALL:

MONEL WETA LNETA GAMA LGAMA=

LAMEDA LLAMBDA EPSILON LEPSILDH /F SLE=0.05:t/

ItPROC UNIVARIATE DATA=ALL PLOT NORMAL FREG;

VAR EFSILON LAMBDA;

PROC CHART DATA=ALL ;

UBAR EPSILON LAMBDA;

RUN?

IIPROC STEPHISE DATA=ALL:

MODEL INVNETA LLAMBDA LEFSILON=

TCHAO VOLCHO P25CHA P5QCHO F75CHO PLODCHE

TCHO1 VOLCHI P25CHI P5ECH1 P75CH1 PLODCH1

TCHO2 VOLCH2 P25CH2 P58CH2 P75CH2 P1EBCH2

TCHB3 VOLCH3 P2SCH3 P5BCH3 P75CH3 PIDOCHS

TCHO4 VOLCHA P2SCHA P5BCH4 P75CH4 P1OBCH4

TCHOS VOLCH5 P25CH5 P58CH5 P75CH5 P1OECH5

LTCHAO LVOLCHO LP2SCHO LP5OCHA LP75CHO LP1AGCHO

LICHO1 LVOLCH1 LP25CH1 LP50CH1 LP75CHI LP1BGCH

LTCHR2 LUOLCH2 LP25CH2 LP5DCH2 LP75CH2 LP1AQCH2

LTCHQS LVOLCHS LP25CHS LP5ACHS LP7SCHS LP1OBCHS

LTCHO4 LVOLCH4 LP2SCH4 LPSACH4 LP75CH4 LP1BOCH4

LTCHOS LVOLCHS LP2SCHS LPSBCHS LP7SCHS LP1ROCHS

INT2SCHQ INTSRCHO INT75CHE INTCHO IF SLE=8.85; 
VAR DIFNETA DIFHET DIFRNETA DIFGNET;

RUN:

IAFROC PLOT DATA= ALL:

PLOT ERROSEPREDS/ VREF $=$ = ;

PLOT ERROZIPREDS/ VREF = :

PLOT ERRO 6 \#REDO / VREF $=0$;

PLOT ERROLBTFREDIB/ UREF $=8$;

FLOT ERROL14TPREDL14/ VREF=8:

PLOT ERROL12TPREDL12/ UREF=?

PLOT ERRO2TFRED2/ UREF $=8$;

RUN!:

ITPROC HEANS N MIN MAX MEAL CV DATA = ALL;

VAR INESC NETA GAMA LAMEDA EPSILON

TCHQO UDLCHO P25CHO P5OCHA FT5CHA P1AQCHQ

TCHO1 VOLCH1 P25CH1 PSQCH1 P75CH PIQQCH1

ICHO2 VOLCH2 P25CH2 P5QCH2 P75CH2 P18BCH2

TCHOS YOLCHS P25CH3 P5ACHS P75CH3 P1GQCHS

TCHO4 VOLCH4 F25CH4 P5OCH4 P75CH4 P1OGCH4

TCHOS VOLCHS P25CHS P5OCHS P7SCHS P1BBCH5;

RUN:

FROC COFE DATA=ALL;

WAF INESC NETA GAMA LAMBDA EPSILON

ICHRO YOLCHE P2SCHO F5ACHO F75CHA PIOBCHE

TCHQ1 VOLCH1 P25CH1 P59CH1 P75CHI PIOBCH1

TCHO2 VOLCH2 P25CH2 P5OCH2 P75CH2 P1BOCH2

TCHOS VOLCHS F25CH3 P5OCHS P75CH3 P1ORCH3

TCHOA VOLCH4 P25CHA P5OCH4 P75CH4 PLOCHA

TCHOS VOLCHE P25CHS F5OCHS F75CHS P1BRCH5;

PROC PLOT DATA = ALL:

PLOT HETANVOLCHO/VREF=G;

FLDT NETAKF2SCH1/VREF $=0$;

PLOT NETAEP 25CH2 $/$ VREF $=0$;

PLOT NETALP2SCHB/VREF $=0$;

PLOT NETALP2SCH4/VREF $=\mathrm{Q}$;

PLOT NETALP25CHS/ VREF $=0$;

PLDT NETALPSECH 1 UREF $=0$ ?

PLOT HETAIPSBCH2/VREF = B;

PLOT HETATPSBCHS NVREF $=$ ?

FLOT HETATPSQCHA/UREF $=8$;

FLOT HETALPSACHS/VREF = :

PLOT HETATF75CHO/VREF=?

PLIT NETATF75CH1/VREF $=0$;

FLOT METATP7SCH2/NREF =0;

PLOT NETATP75CHS/ VREF =0; 


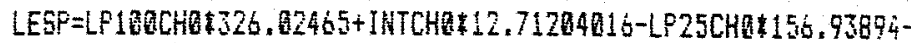
iNTSCHA12.66920615;

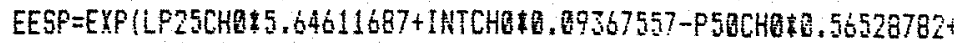
INT25CHD* . 01789580):

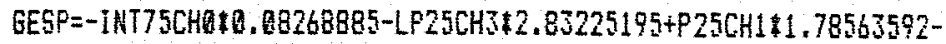
LP5OCH1 $12.10854852 ;$

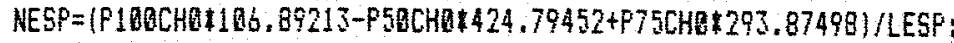

ITDATA TOTAL: MERGE ALL VAZAO: BY NH:

ARRAY FIE(1DO) FIEI-FIEIBB;

ARRAY FHE (IBR) FHEI-FHEIOB;

MAXE $=1$ NT $(L E S P / 20):$

DO I I TO KAYY :

$Y E=(1 / 2 Q-E E S P) / L E S P:$

ISIHHYE $=$ LOG YYE+SORT(YETI2+1));

FIE [ I =PRORNDRM(GESP+NESPIISINHYE);

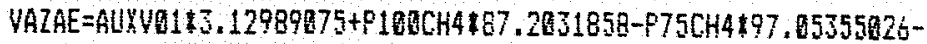
AllXVQ4 41.29634887 ;

IF I GT 1 THEN FHE(I) $=(F I E(1)-F Z E(1-1))$ WVAZAE;

ELSE FWE(I)=FIE(I) VAIAE;

END;

PROC FRINT:TITLE1 'SINULACAO OA VAZAO DA MICROEACIA DE CUNHA'; TITLE2 'SISTEMA UTILIZADO : SU';

WAR FWEI-FHEIOR :

FORMAT FHE1-FHE1BO 6.2

PROC PRINT ;

VAR LAMEDA LESF EPSILON EESP GAKG GESP NETA NESP VAZAC VAZAE:

FORHAT LAMBDA LESP EPSILON EESP GAMA GESP HETA NESP VALAC VAZAE $6.2 ; \%$

IFPRC REG DATA=ALL:

MODEL NETA=TCHO1 VOLCHS YOLCHA P25CH4 P5BCHS LUOLCHS/NOINT: OUTPUT OLIT=DIFNETA P=PREDHETA \&

IFFOC REG DATA=ALL;

MODEL 113 = PIOACHO PSACHO P7SCHO/NOINT P:

IODATA ALL:

MEREE ALL DIFWETA DIFHET;
DIFNETA = PREDNETA-NETA:
DIFRNETH = DIFHETA/HETA:
DIFNET = PREDHET-NETA;
DIFRET = DIFHET/HETA;

PFDC MEANS N MIN MAX HEAN CU USS: 
LESP $=$ LP1BACHQ $326.02465+1$ NTCHE $12.71204016-1$ P25CHO\$156.93894INT58CHOt2.60920615;

EESP=EXP(LP25CHO45.64611687+ INTCHO40.89367557-P58CHOtQ. 56528782+ INT25CHe*0. 01709588 );

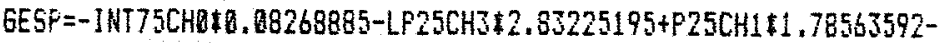
LP58CH12.10854852;

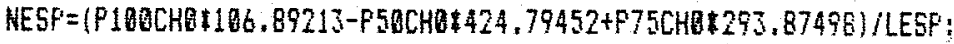

IDDATA TOTAL; MERGE ALL VAZAO; BY NH: ARRAY FIE⿱DEQE FIE1-FIEIOQ;

ARRAY FWEd10QE FUEI-FWE1OQ:

MAXE=INT(LESF/20):

DO I = TO RAYE;

$Y E=\{(120-E E S P] / L E S F ;$

ISINHYE $=$ LOG $(Y E+$ SQRT $(Y E \pm \div 2+1))$;

FZEAI =FRDBNDRY ( GESP+NESP IISINHYE):

VAIAE =AUYVO113.12989075+P100CH4\$87.2031858-P75CH4197.85355026AliuQ441.29634887;

IF I GT 1 THEN FHEaIE=(FIEdIE-FLEaI-1) WVAZAE;

ELSE FHEaIE=FIEdIEZVAZAE:

END;

FROC PRINT:TITLEJ 'SIMULACAO DA VAZAO DA MICROBACIA DE CUNHA';

TITLE2 SISTEHA UTILILADO ; SU';

UAR FWE1-FHE1QR ;

FORMAT FHEI-FWEIOQ 6.2 ;

FROC PRINT;

VAR LAMBDA LESP EFSILON EESP GAMA GESP NETA NESF VALAC VAZAE;

FORMAT LAMBDA LESF EFSILOH EESF GAMA GESP NETA MESF VAZAC VAZAEE 6.2; $/$

ITFROC REG DATA=ALL;

MODEL NETA=TCHO1 VOLCH3 VOLCH4 P25CH4 P5OCH5 LVOLCHS/NOINT;

OUTPUT OUT=DIFNETA P=PREDNETA : II

I PPROC REE DATA=ALL:

MODEL YI\} = PIOCCHQ PSQCHE P7SCHB/NOINT P:t/

ITDATA ALL:

MERGE ALL DIFNETA DIFNET;

DIFHETA = PREDNETA-NETA;

DIFRNETA = DIFNETA/NETA:

DIFNET = PREDNET-NETA:

DIFRNET = DIFNET/NET

PROC MEAHE N MIN MAX MEAN CU USS: 


$\begin{array}{lllllll}82 & 0.000 & 0.000 & 0.000 & 0.000 & 0.000 & 0.000 \\ 82 & 0.000 & 0.000 & 0.000 & 0.000 & 0.000 & 0.006 \\ 82 & 0.000 & 0.090 & 0.000 & 0.000 & 0.000 & 0.000\end{array}$

FROC SORT DATA = CHLUAR: BY NH:

RUN:

Calculo dos parametros do Sist. Johnson de Distr.

Calculo das probalilidades do Sist. Johnson de Distr.

Calculo das equacoes re rearessao /stepwise/residuos e plots.

DATA HIDROG:

INFILE CARDS MISSOUER:

IIFUT $1 \times 2,0$

RETAIN NH:

IF $X$ WE , THEN DO;

$\mathrm{NH}=\mathrm{h}$

$\mathrm{N}=0$

END?

$N+1$

SELECT(N):

WHEN(1) INPUT Q3 V1 -V11;

WHEN(2) INPUT 3 V12-V22;

WHEN(O) INPUT QS V23-153:

WHEN(4) INPUT GS VR4-V44;

WHEN(5) INPUT Q Q V45-U55;

WHEN(6) INPUT QS V56-V66:

WHEV(7) INPUT QJ V67-V70:

OTHERHISE:

END;

CARDS:

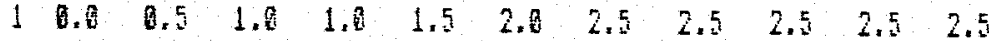

$2.5 \quad 2.5 \quad 3.6 \quad 3.0 \quad 3.5 \quad 3.5 \quad 4.8 \quad 4.0 \quad 4.8 \quad 4.6 \quad 4.0$

4.0 $4.0 \quad 4.0 \quad 4.0 \quad 4.5 \quad 3.0 \quad 3.0 \quad 3.0 \quad 3.6 \quad 3.0 \quad 3.0$

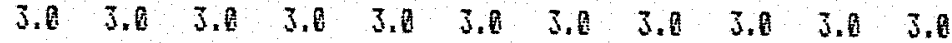

$3.6 \quad 3.6 \quad 3.6 \quad 2.5 \quad 2.5 \quad 2.5 \quad 2.0 \quad 2.0 \quad 2.0 \quad 2.0 \quad 2.0$

$2.02 .6 \quad 2.6 \quad 2.0 \quad 2.0 \quad 1.5 \quad 1.5 \quad 1.5 \quad 1.5 \quad 1.5 \quad 1.5$

1.5 0.0

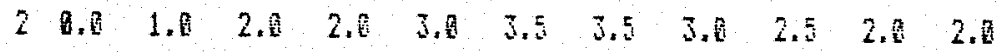

1.51 .51 .01 .01 .01 .61 .61 .01 .61 .01 .0

Q.5 $\quad 0.0$

30.01 .01 .0 1.0 1.0 1.8. 0.0

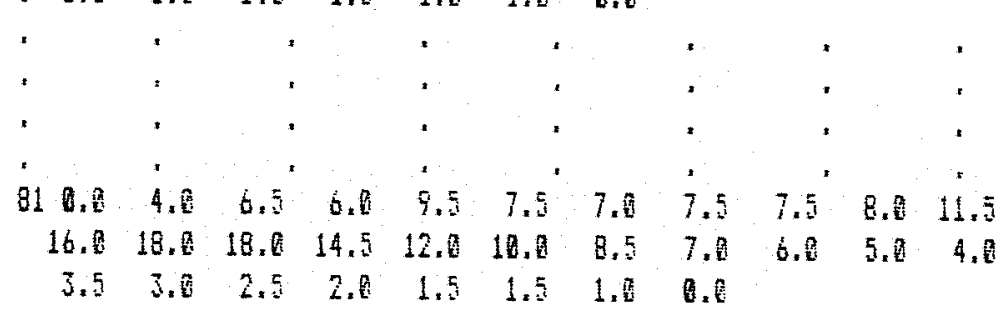




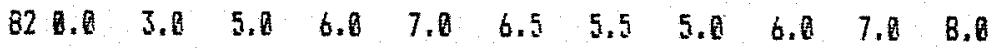

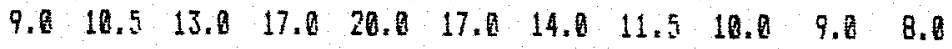

$\begin{array}{llllllllll}7.8 & 6.0 & 5.0 & 4.0 & 3.5 & 3.0 & 3.0 & 2.5 & 0.6\end{array}$

;

FROC SORT:

BY NH:

PROC SUMMARY,

BY NH:

VAR V1-V7Q:

OUTPUT OUT $=$ HIDROG (DROF $=$ TYFE_ FREQ_ SUM $=$;

IPROC PRINT:

4 TITLE2 ' $t$ CARDS $\mathbf{W}$;

DATA HIDROG:

ARRAY V(7Q) V1-V7D:

ARRAY VAC(70):

ARRAY PY(5):

ARRAY X(4);

MERGE HIDROG HIDUAR (KEEP=NH EPSILON LAKBDA);

BY NH;

VAZAC=SUM (OF VI-V7Q);

DMIN=?99?:

REIAIN AUXI;

RETAIN AUXEPS;

RETAIN AUXLAMB;

RETAIN AUXNET;

RETAIN AUXGAM:

DO $Z=0.01$ TO 1 BY Q.81:

PY1=PRORNORH $(-3$ ZZ) *VAZAC:

PYZ=PROBNORH(-I) NVAZAC;

PYZ=PROBNORH(7) WAZAC:

PY4=PROBNORM(3\%1) VVAZAC;

VAC(1) $=V(1)$,

$k=1 ;$

Do $1=1$ TO $70 ;$

VAC $(1+1)=$ SUM $(V A C(1), V(1+1))$,

Do $3=1$ To $1+1$

$T=204(\mathrm{~J}-1)$;

IF PY(K) YVACO) THEN DO:

$X(K)=(T-20)+$ SUM $(P Y(K),-V A C(J-1))+20 / V(J))$

$\mathrm{J}=\mathrm{I}+1$;

IF $k=4$ THEN $1=70$ :

$k=k+1$

END:

END;

EHI: 


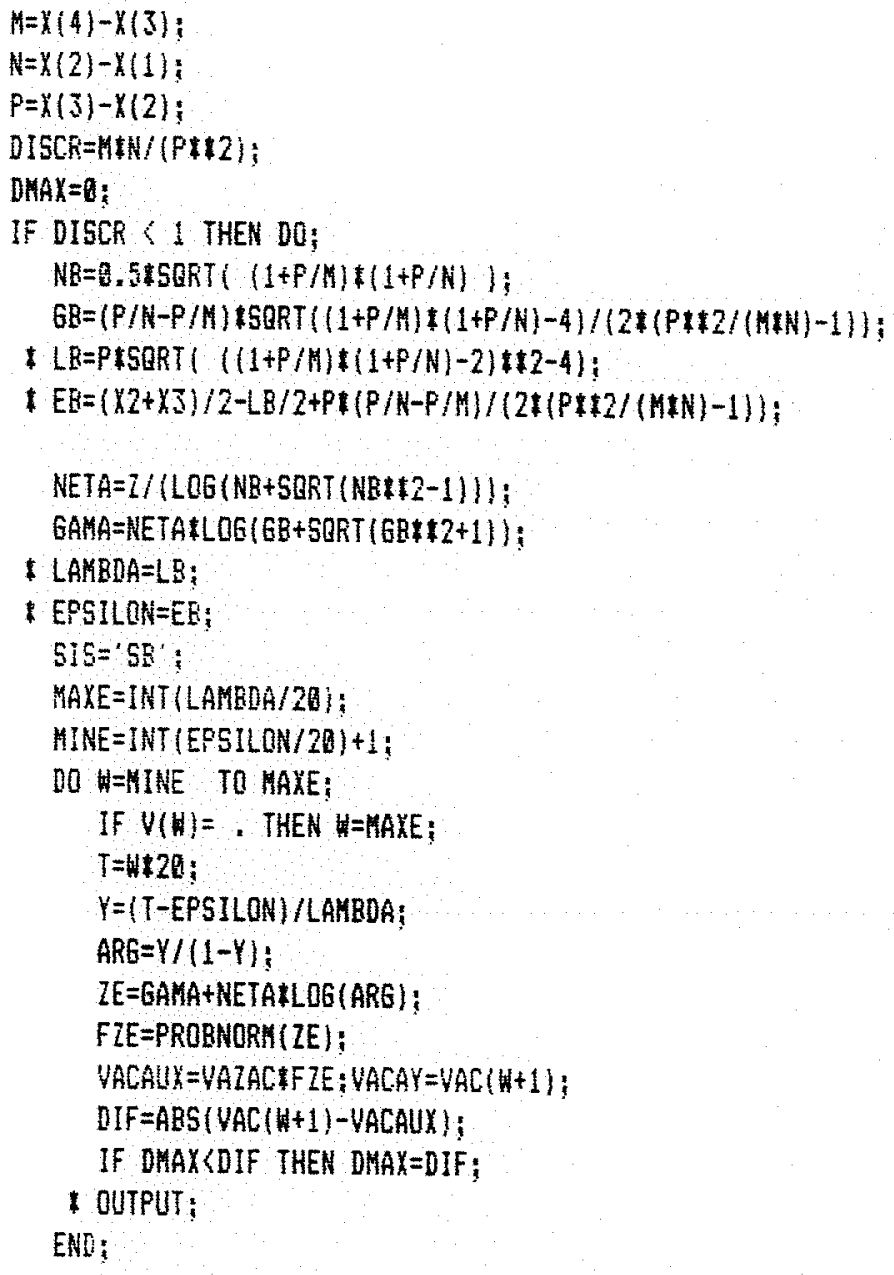




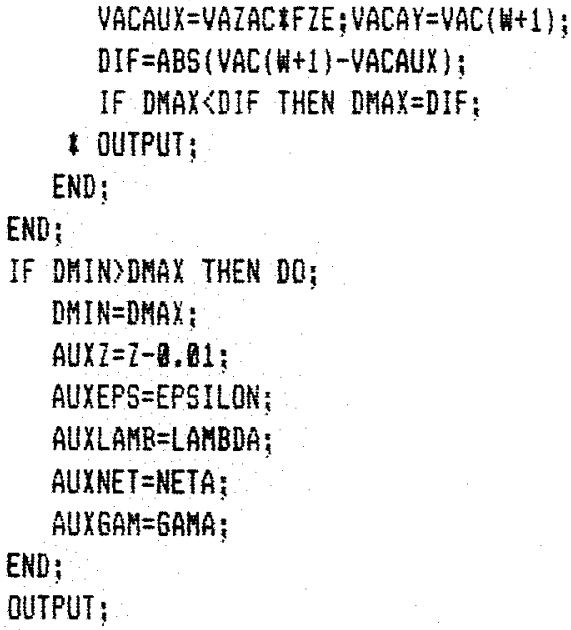

VAR WH $Z$ W T EPSILOH LAHBDA NETA GAHA Y ARG IE FIE VACAUX VACAY DIF:

FORMAT $Z$ NETA GAMA Y ARG IE FIE VACAUX VACAY $6.2: 4$ !

PROC UNIUARIATE FLOT NORMAL FREQ:

$$
\text { VAR DYIN; }
$$

1 BY NH:

PRDC SORT;

BY NH:

DATA HIDROG;

SET HIDROG:

BY NH:

IF LAST.NH:

PROC PRINT ;

VAR NH 2 DISCR SIS DMAX DHIN AUXI AUXEPS AUXLAME AUXNET AUXGAW:

FORHAT $Z$ DISCE 6.2 DMAX DHIN AUKZ AUXEFS AUXLANB AUXEET AUKGAOH 6.2 ; 11

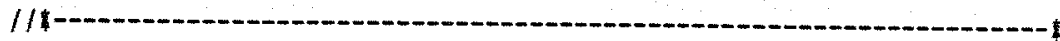

Leitura Variayeis do Sist, Johnson de Distrib-...-...-.

DATA HIDVAR;

INFUT NH 10-11 INESC 12-20 NETA 22-30 6AMA 32-40 LAMBDA 41-50 EFSILON 51-68; CARDS:

\begin{tabular}{|c|c|c|c|c|c|}
\hline 1 & 0.667 & 0.465 & 0.138 & 1360.0 & 40.0 \\
\hline 2 & 3.667 & 0.589 & 0.529 & 440.0 & 220.0 \\
\hline 3 & 1.383 & 2.065 & 7.239 & 109.6 & $8 \mathrm{~B}, \mathrm{a}$ \\
\hline & * & . & . & . & . \\
\hline & . & , & . & $\therefore$ & . \\
\hline &. & . &. &. & $\star$ \\
\hline & 0.667 & 1.250 & -3.452 & 500.9 & 40.0 \\
\hline & 2.667 & 1.173 & -0.837 & 560.0 & 160.0 \\
\hline & 1.800 & 1.825 & 0.060 & 580.0 & 60.0 \\
\hline
\end{tabular}

PROC SORT DATA = HIDVAR:

BY NH: RUL: 
DATA ALL, NEREE HIDUAR CHUVAR ; BY NH;

IF $N H=48$ THEN OELETE:

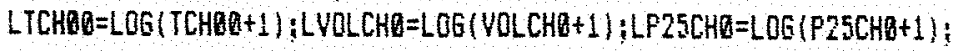

$L T C H O 1=L O 6(T C H O 1+1)$, LVOLCH $=L O G($ VOLCH +1$), L P 25 C H 1=L O 6(P 25 C H 1+1)$;

$L T C H O 2=L O G(T C H 22+1), L$ VOLCH $2=L O G($ VOLCH $2+1), L P 25 C H 2=L O S(P 25 C H 2+1) ;$

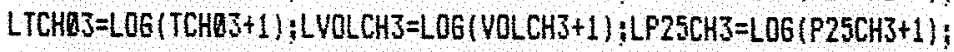

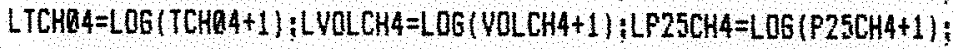

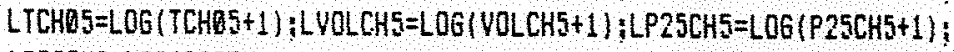

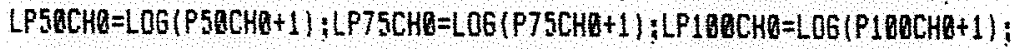

$\angle P 50 C H 1=L Q 6(P 5 O C H 1+1), L P 75 C H 1=L 06(P 75 C H 1+1), L P 100 C H 1=L 06(P 100 C H 1+1)$

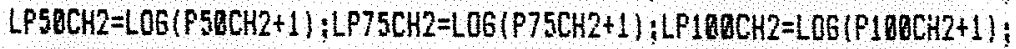

$\angle P 50 C H 3=\angle O G(P 5 A C H 3+1), \angle P 75 C H 3=\angle 06(P 75 C H 3+1), L P 100 C H 3=\angle 0 G(P 100 C H 3+1)$

LP5OCH4=LOG(F5OCH4+1), LP75CH4=LOG(P75CH4+1),LP10OCH4=LOG(P10OCH4+1):

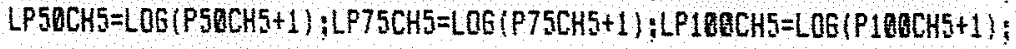

AUX1=P1OOCHO/LAMBDA:

AUK2= F 7 SCHD/LAFBDE:

AUX $3=$ P5ECHO $/ \mathrm{LAMBDA}$

ALIXA=F25CHB/LAMBOA

$A L X \cup A R I=P 10 O C H 4+12$;

AUXUAR2 $=P 75 C H 4112$;

AUXUART=P75CH4EP100CH4:

AUXVAR4 $=\angle T C H D 1 \& \$ 2$;

AUXVARS=L UOLCH1 $\mathrm{t}+2$;

AUXVAR6=LTCHOL $L$ UDLCHI;

AUXVBI= $=O L C H B+V O L C H 1$;

AUXVO2= $\mathrm{OOLCHO}+\mathrm{VOLCH}$;

AUXVBZ=VOLCHO+VOLCH3,

AUXVO $4=$ VOLCHQ + VOLCH4 ;

AUXUDS=VOLCHO+VDLCHE:

AUXVOG $=$ VOLCHG-VOLCHI)

AUXVO7=VOLCHD- $\mathrm{VDLCH} 2$

AUKVOB=VOLCHO- UOLCHS,

AUXVA9= VOLCHA-VOLCHA ;

AUXV1O=VOLCHA-UDLCH5:

AUXV $11=$ VOLCHDE 2 ;

AUXVI7= UOLCH9) 10.5 !

LINESC=LO6 (INESC+1) ILUETA=LOG(NETA+1),LLAMBDA=LOG (LAMBDA);

$\angle E P S I L O N=\angle O G$ (EPSILON), LGAKA=LOG(GAMA+1B)

INUNETA=1/HETA;

$Y 1$ =INESCINETA:

$Y 2$ =INESC $/$ LABBDA,

$Y 3$ =INESC/ (NETA+LAMBDA):

$Y 4=$ INESC/ (NETA-LAMBDA),

$Y 5$ = IIESC (NETALLAHBDA)

I6 =INESC/ (NETA+LAMBDA+1NESC)

$Y 7$ =NETA/INESC,

YQ =NETA/LAKBDA:

$Y 9=N E T A /(I N E S C+\angle A M B D A)$,

Y1B =NETA / (INESC-LAMBDA):

Y11 =NETA/ (INESCLLAMBDA): 
Y12 =NETA/ (INESC+LAMRDA+NETA),

$Y 13$ =LAMBDA/NETA;

$Y 14=\angle A M B D A / I N E S C$;

$Y 15$ =LAKBDA/ (NETA+INESC) ;

$Y 16$ =LAMRDA/ (NETA-INESC) /

Y17 =LAMBDA/ (METALINESC),

Y1B =LAKBDA/ (HETA+INESC+LAMBDA);

INTCHO=VOLCHO/P10BCHO;

INT25CHO=VOLCHO/P25CHO;

INT75CHQ=VDLCHO/P75CHO;

INT 5OCHQVVOLCHO/P SOCHO:

$71=1 / Y_{1}$

$22=1 / Y 2$

$13=1 / Y 3$

$14=1 / 14:$

$75=1 / 15$

$16=1 / 16$

$27=1 / 17$;

$28=1 / 18$

$79=1 / 49$

$210=1 / 101$

$Z 11=1 / Y 11:$

$212=1 / 12 ;$

$213=1 / 15$

$714=1 / 714$

$215=1 / 415$

$716=1 / 716$ :

$717=1 / 1 / 17$

$218=1 / 118$

YINESC=IMESL;

LY1 =LOG(ABS (INESC $/$ NETA));

$L Y_{2}=\angle O G$ (ABS (INESC/LAMBDA)/;

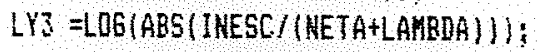

LYA =LOG (ABSI INESC / (NETA-LAMBDA)I),

LYS = LOG (ABSIINESC/ INETAKLAMBDA) I)!

$\angle Y_{6}=$ LLGI ABS I INESC/ I WETA+LAMBDA+INESCI)/।

LY7 = L0G(ABS(NETA/INESC)),

LYB = LOG (ABSI (KETA/LAMBDA));

LY9 =LOG(ABSINETA / INESC+LAMBDA) I),

LY10 =LOGIABSINETA / INESC-LAMBDA II);

LY11 =LOG IABSINETA / IINESCLLAMBDA I)।;

LY12 =LOG (ABSINETA / INESC+LAMBDA+NETA)I):

LY13 = LOGIARSILARBDA/NETA) I,

LY14 =LOG (ABSILAKBDA/IMESC) I;

LY15 = LOG (ABSILAMBDA / INETA+INESCI)I)

LY 16 =LOG (ARSILAMBDA / WETA-INESC) I),

LY17 =LOGIABSILAMEDA/ (NETAEIWESC) ) ),

LY1B = LOG/ABSILAMBDA/ (NETA+INESC+LAKBDA)I); 